\title{
Histomorphologische Kriterien für zytogenetisches Risiko beim myelodysplastischen Syndrom
}

\author{
INAUGURAL-DISSERTATION \\ zur Erlangung des Doktorgrades \\ der Medizinischen Fakultät der \\ Georg-August-Universität zu Göttingen
}

vorgelegt von

Cornelius Kürten

aus

Düsseldorf

Göttingen 2017 
Dekan:

Referent/in

Ko-Referent/in:

Drittreferent/in:
Prof. Dr. rer. nat. H. K. Kroemer

Prof. Dr. med. P. Ströbel

PD Dr. med. J. Schanz

Prof. Dr. med. M. Oppermann

Datum der mündlichen Prüfung: 15. Mai 2018 
Hiermit erkläre ich, die Dissertation mit dem Titel "Histomorphologische Kriterien für zytogenetisches Risiko beim myelodysplastischen Syndrom" eigenständig angefertigt und keine anderen als die von mir angegebenen Quellen und Hilfsmittel verwendet zu haben.

Göttingen, den 


\section{Inhaltsverzeichnis}

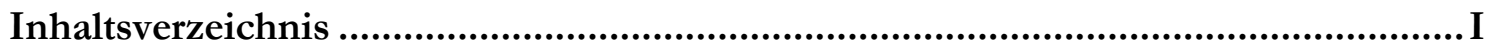

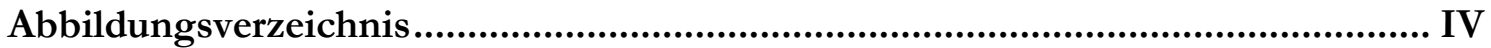

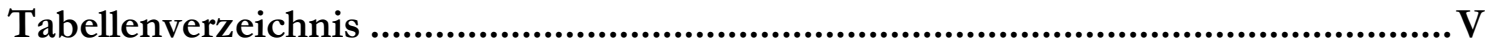

Abkürzungsverzeichnis ......................................................................................... VI

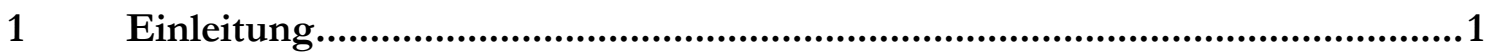

$1.1 \quad$ Hämatopoiese und Knochenmark .......................................................................................

1.1.1 Entwicklung der adulten Hämatopoiese _............................................................................1

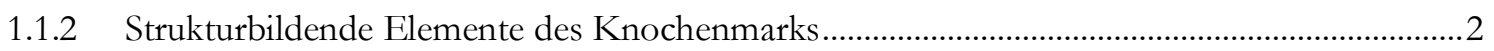

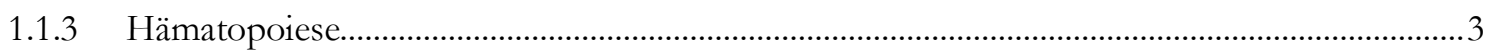

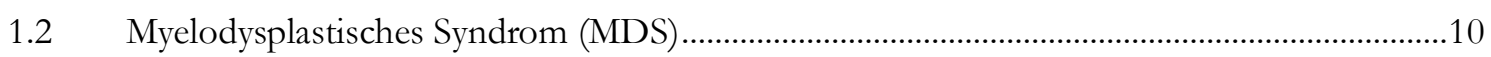

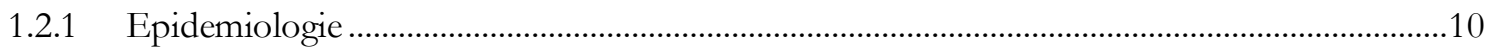

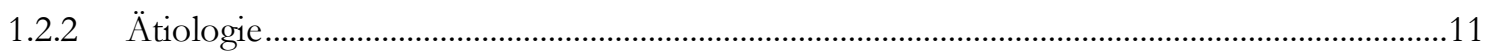

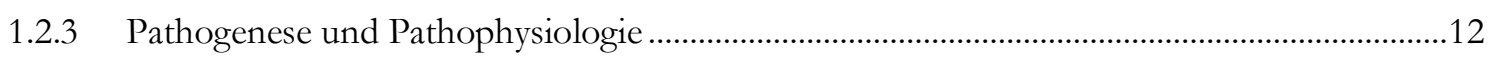

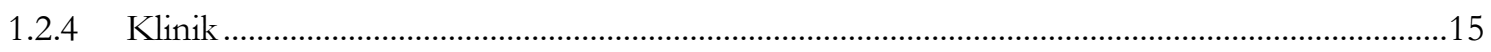

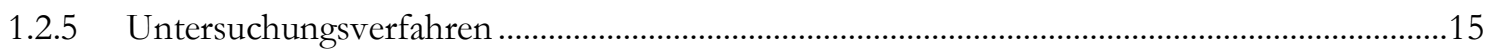

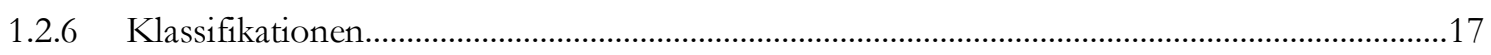

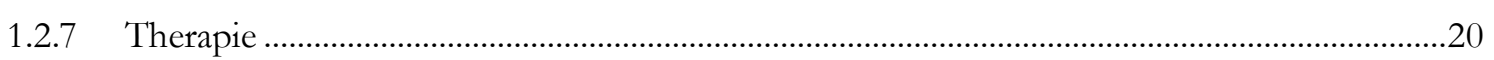

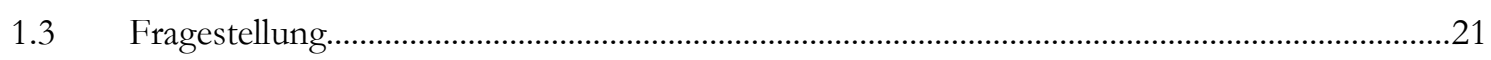

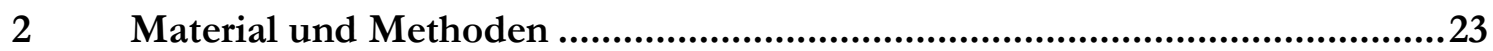

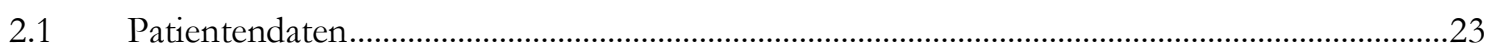

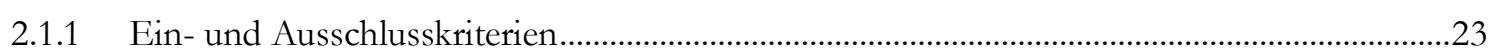

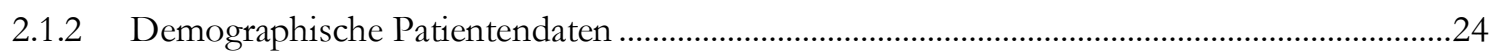

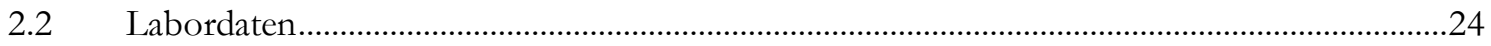

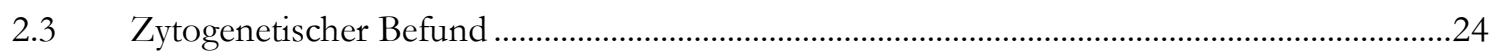

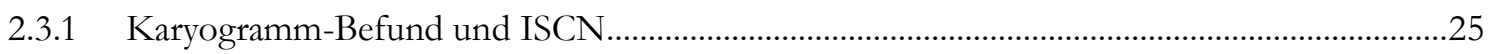

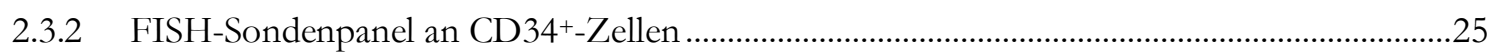

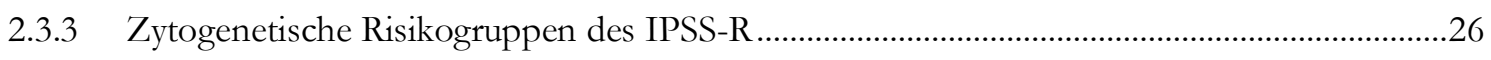

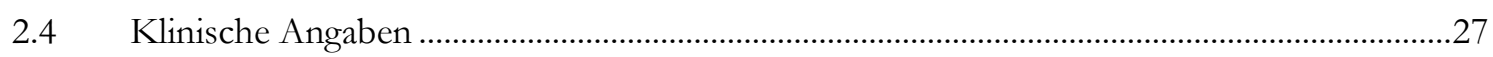

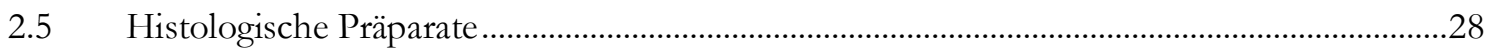

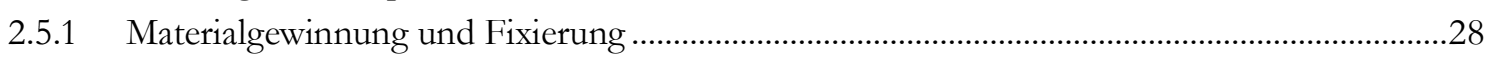

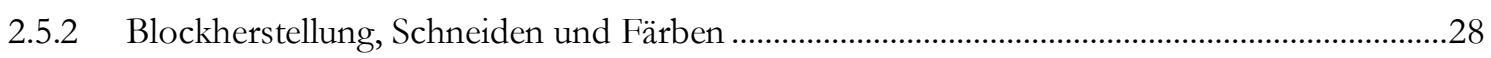

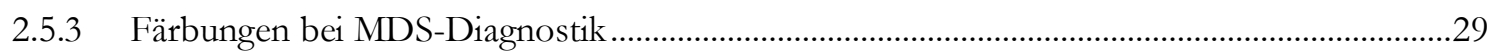

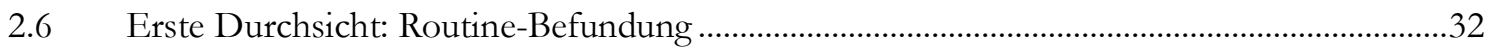

2.6.1 Gesamt-Zellularität und Alterskorrektur................................................................................

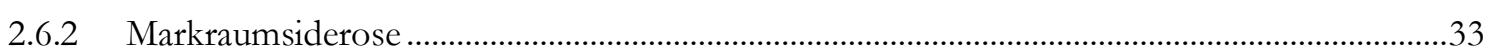

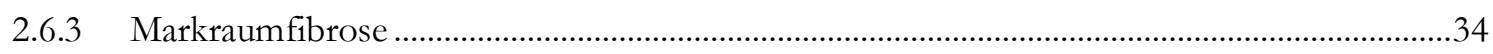




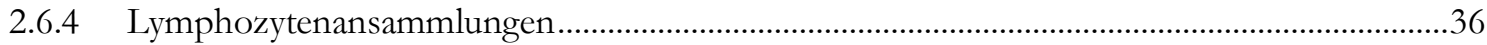

2.6.5 Qualitative morphologische Beurteilung der Hämatopoiese.................................................36

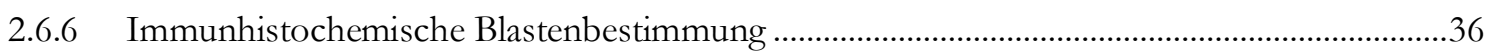

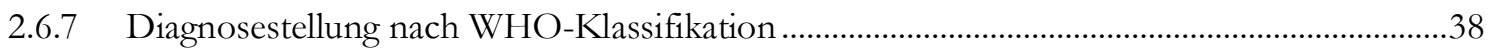

2.7 Zweite Durchsicht: Anwendung des Morphologie-Scores....................................................39

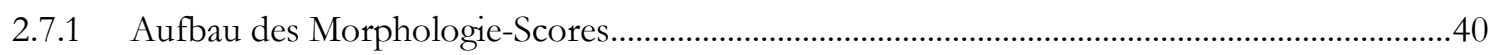

2.7.2 Quantitative Veränderungen einzelner Zellreihen ....................................................................41

2.7.3 Reifungs- und Architekturstörung der Erythropoiese..............................................................4 41

2.7.4 Reifungs- und Architekturstörung der Megakaryopoiese .......................................................43

2.7.5 Reifungs- und Architekturstörung der Granulopoiese ..............................................................45

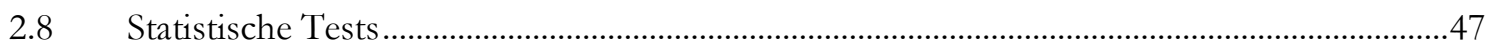

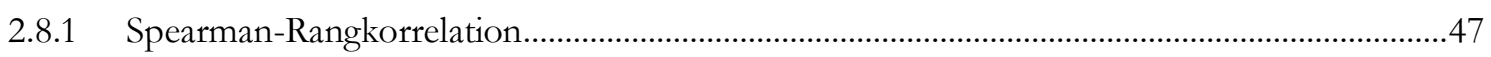

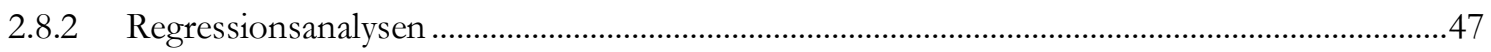

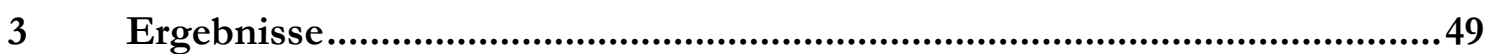

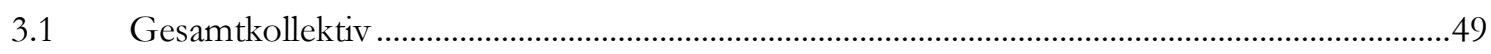

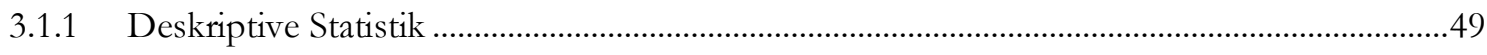

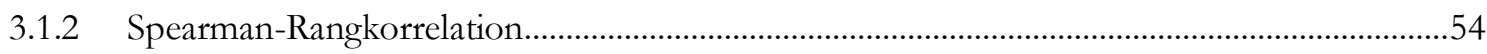

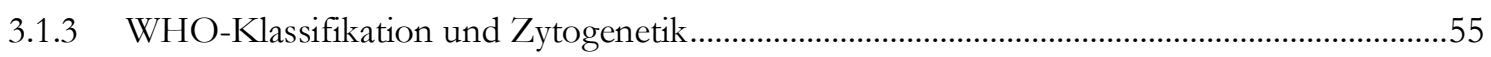

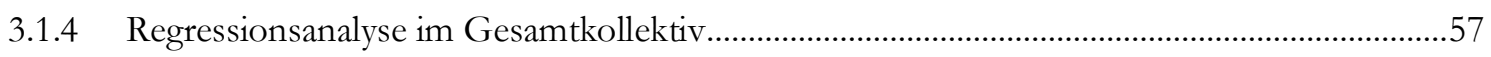

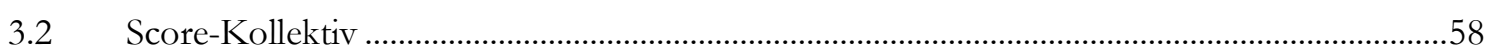

3.2.1 Vergleich von Gesamt- und Score-Kollektiv ........................................................................58

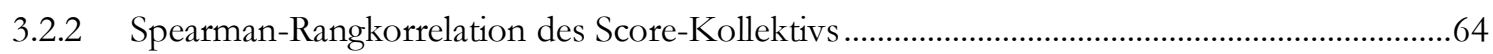

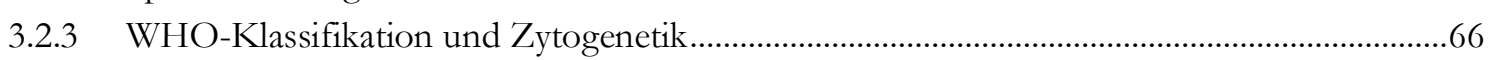

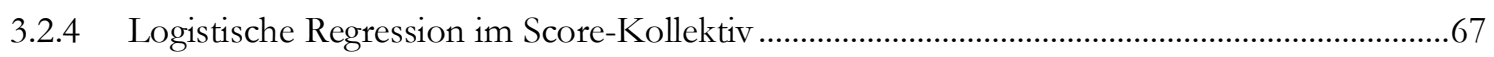

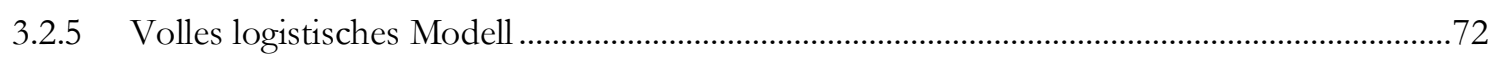

$4 \quad$ Diskussion..........................................................................................74

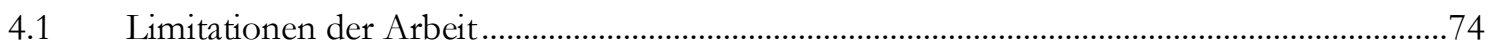

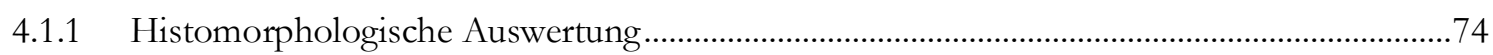

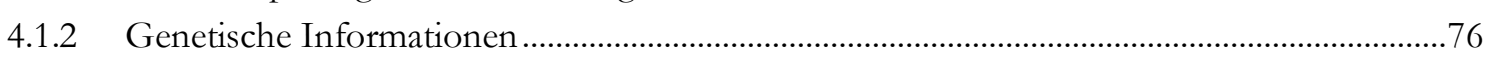

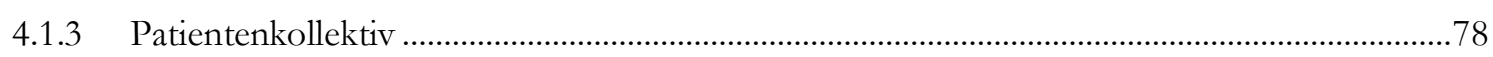

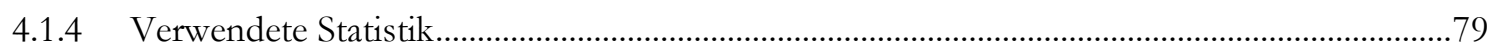

4.2 Zusammenhang zwischen Histologie und WHO-Kriterien ...................................................8 80

4.3 Zusammenhang zwischen Histologie und Zytogenetik ......................................................81

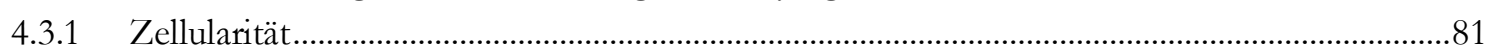

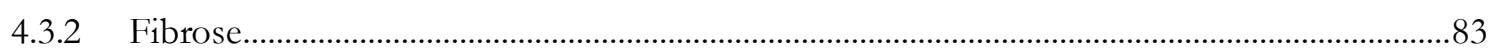

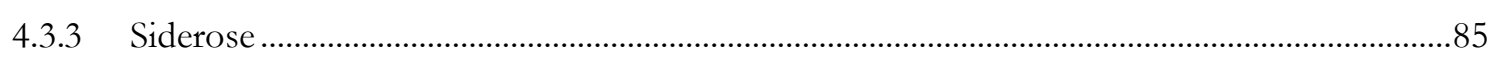

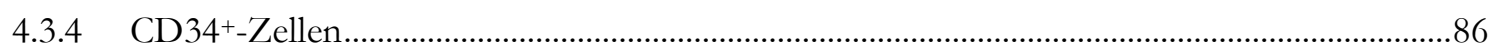

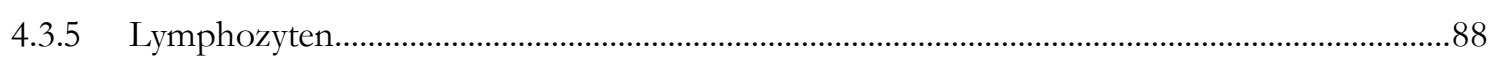

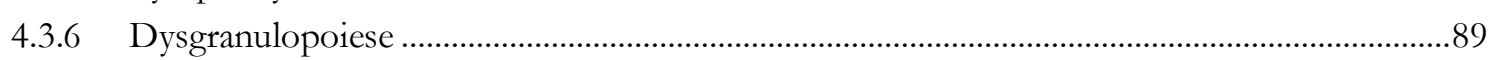

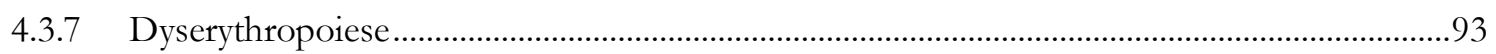

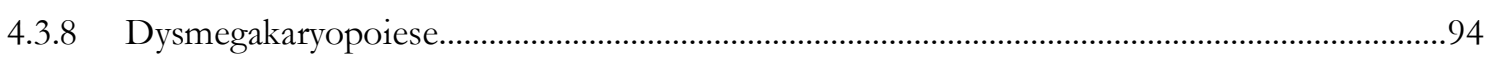

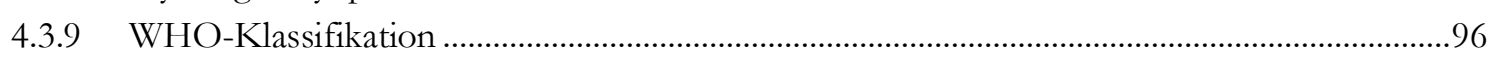

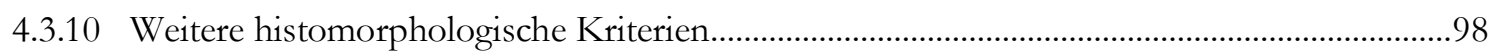


4.4 Pathophysiologisches Erklärungsmodell ..............................................................................99

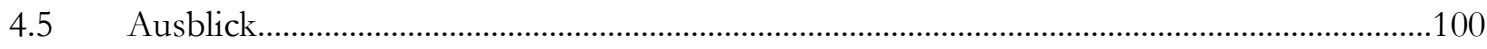

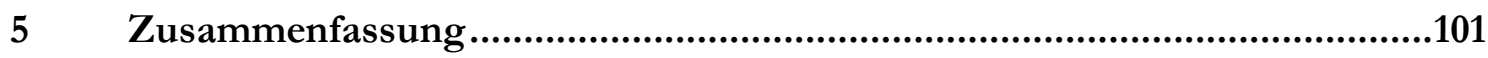

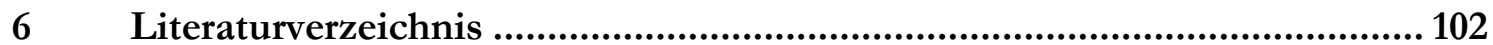




\section{Abbildungsverzeichnis}

Abbildung 1: Strukturbildende Elemente des Knochenmarks ..........................................................3

Abbildung 2: Modell der hierarchisch organisierten Hämatopoese ....................................................4

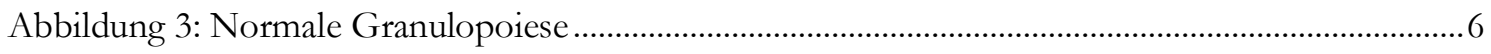

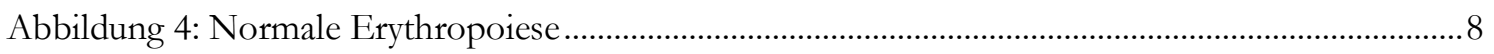

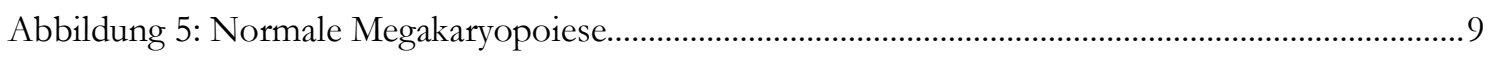

Abbildung 6: Zusammenhang der Untersuchungsmethoden bei MDS ............................................22

Abbildung 7: Studienaufbau, Ein-/Ausschlusskriterien, Kollektive....................................................24

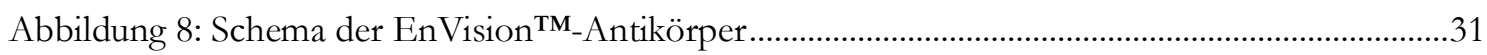

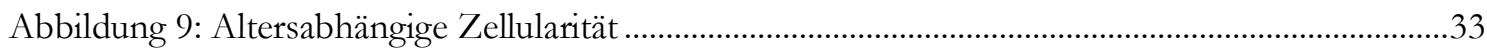

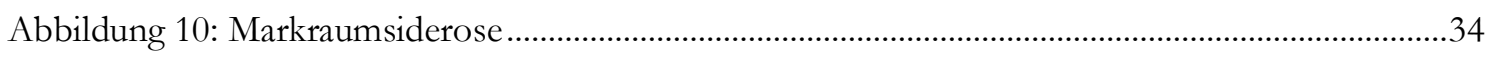

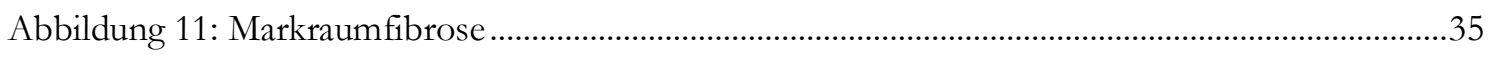

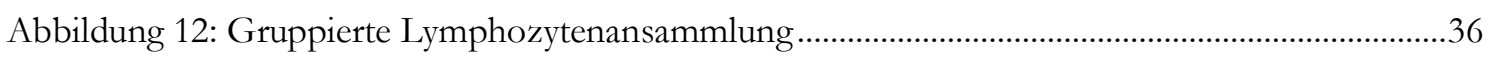

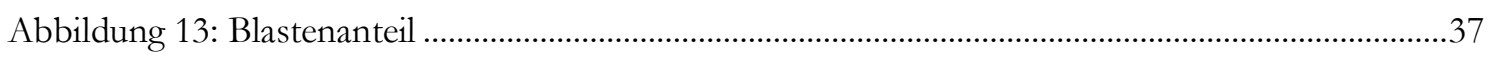

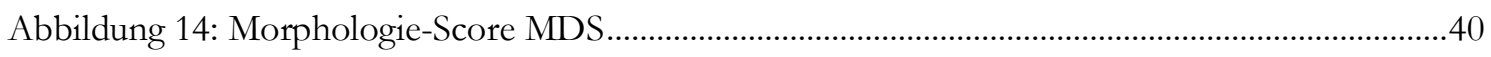

Abbildung 15: Quantitative Veränderungen der Zellreihen ..............................................................41

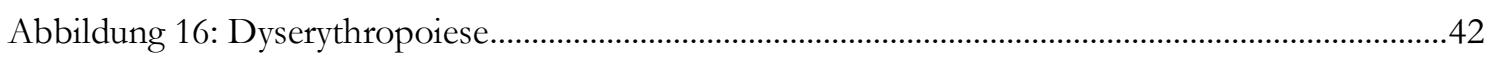

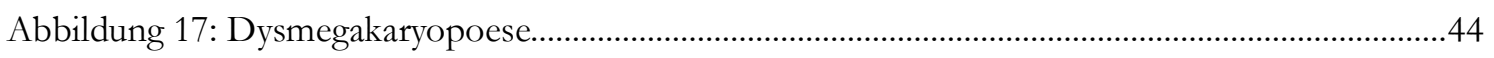

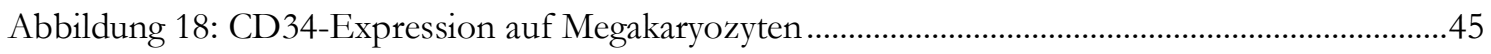

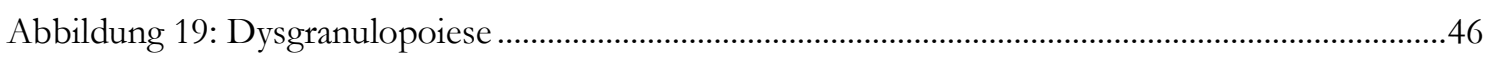

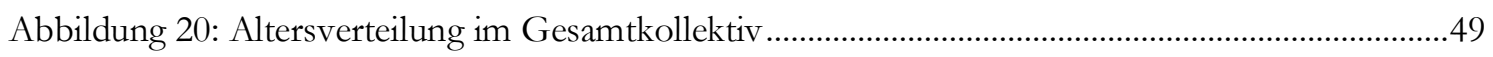

Abbildung 21: Zytogenetische Risikogruppen (nach IPSS-R) ..............................................................50

Abbildung 22: Anzahl der bewerteten Metaphasen im Gesamtkollektiv .............................................51

Abbildung 23: Morphologische Veränderungen der Routinediagnostik .............................................53

Abbildung 24: Immunhistochemie im Gesamtkollektiv ..................................................................54

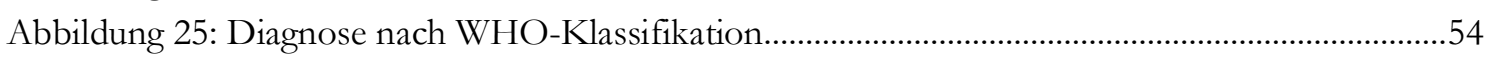

Abbildung 26: IPSS-R Risikogruppen in der WHO-Klassifikation Gesamt-Kollektiv .......................56

Abbildung 27: Altersverteilung, Zytogenetik und Routinediagnostik im Score-Kollektiv .................61

Abbildung 28: Immunhistochemie im Score-Kollektiv ............................................................................61

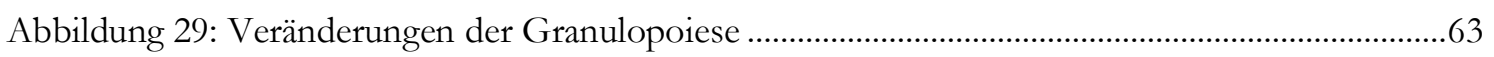

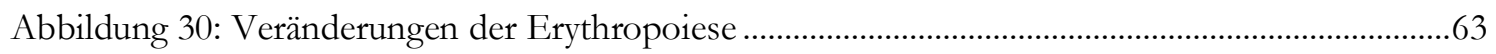

Abbildung 31: Veränderungen der Megakaryopoiese ...................................................................................64

Abbildung 32: IPSS-R-Risikogruppen in der WHO-Klassifikation des Score-Kollektivs..................67 


\section{Tabellenverzeichnis}

Tabelle 1: Kriterien des IPSS 19

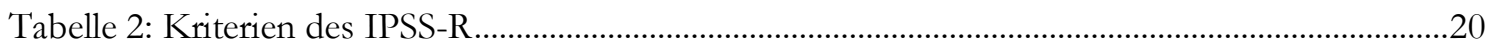

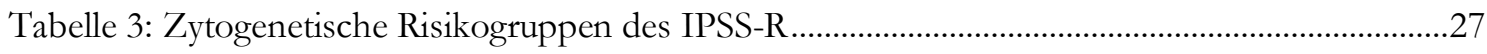

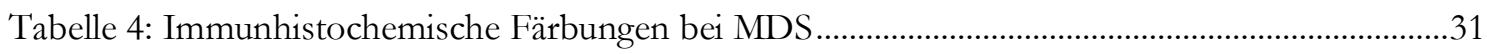

Tabelle 5: Normale Zellularität nach europäischem Konsensus ...........................................................332

Tabelle 6: Einteilung der Markraumfibrose nach europäischem Konsensus......................................35

Tabelle 7: Befunde im peripheren Blut und Knochenmark, WHO-Klassifikation 2016....................39

Tabelle 8: Charakteristika von Patienten mit weniger als 15 Metaphasen...........................................51

Tabelle 9: Bestimmung der Genetik mittels pB-/KM-FISH.............................................................52

Tabelle 10: Spearman-Rangkorrelation von Parametern der ersten Durchsicht .................................55

Tabelle 11: Regression des IPSS-R mit dem Prädiktor WHO-Subtyp im Gesamtkollektiv................56

Tabelle 12: Univariate Regression des IPPS-R mit Prädiktoren der Routineparameter......................57

Tabelle 13: Ordinale logistische Regression des IPPS-R im Gesamtkollektiv.....................................58

Tabelle 14: Exakter Test nach Fisher zum Vergleich von Anteilen ..................................................59

Tabelle 15: T-Test zum Vergleich metrischer Variablen.........................................................................60

Tabelle 16: Spearman-Rangkorrelation zwischen Routineparametern und Endpunkten ...................65

Tabelle 17: Spearman-Rangkorrelation zwischen Morphologiescore und Endpunkten.....................66

Tabelle 18: Regression des IPSS-R mit dem Prädiktor WHO-Subtyp im Score-Kollektiv ................67

Tabelle 19: Univariate Regression des IPPS-R mit Prädiktoren der Granulopoiese ...........................68

Tabelle 20: Ordinale logistische Regression des IPSS-R mit Variablen der Granulopoiese...............69

Tabelle 21: Univariate Regression des IPPS-R mit Prädiktoren der Erythropoiese .............................70

Tabelle 22: Ordinale logistische Regression des IPSS-R mit Variablen der Erythropoiese..................70

Tabelle 23: Univariate Regression des IPPS-R mit Prädiktoren der Megakaryopoiese........................71

Tabelle 24: Ordinale logistische Regression des IPSS-R mit Variablen der Megakaryopoiese ............72

Tabelle 25: Volles Modell der ordinalen logistische Regression des IPSS-R .......................................73 


\section{Abkürzungsverzeichnis}

AA

ALIP

AMA-CD34

AML

CAE

CBA

CLP

CMML

CMP

$\mathrm{DAB}$

FAB

FISH

GMP

$\mathrm{HE}$

HRP

HSZ

ID

IPSS

IPSS-R

ISCN

KI

$\mathrm{LDH}$

MALT

$\mathrm{MCV}$

MDS

MDS-EB

MDS-MLD

MDS-SLD

MDS-U

MEP

$\mathrm{MF}$

MPA

MPG

$\mathrm{MPO}$

OR

PAS

PMF

$\mathrm{PNH}$

RA

RAEB

RAEB-T

RARS

RCMD aplastische Anämie

abnormal localization of immature precursors

abnormal multifocal accumulation (clustering) of $C D 34^{+}$precursor cells

akute myeloische Leukämie

Chloracetatesterase

Chromosomen-Bänderungs-Analyse

common lymphoid progenitor

chronic myelomonocytic leukemia

common myeloid progenitor

3,3'-Diaminobenzidin Tetrahydrochlorid

French-American-British

Fluoreszenz-in-situ-Hybridisierung

granulocyte/macrophage progenitor

Hämatoxylin-Eosin

horseradish peroxidase

hämatopoietische Stammzellen

Identifikationsnummer

International Prognostic Scoring System

International Prognostic Scoring System (Revised)

International System for Human Cytogenetic Nomenclature

Konfidenzintervall

Laktat-Dehydrogenase

mucosa associated lymphoid tissue

mean corpuscular volume

myelodysplastisches Syndrom

MDS with excess of blasts

MDS with multilineage dysplasia

MDS with single lineage dysplasia

MDS, unclassifiable

megakaryocyte/ erythrocyte progenitor

Markraumfibrose

Metaphasen abnorm

Metaphasen gesamt

Myeloperoxidase

Odds Ratio

periodic acid-Schiff

primäre Myelofibrose

paroxysmale nocturnale Hämaturie

refractory anemia

refractory anemia with excess blasts

refractory anemia with excess blasts in transformation

refractory anemia with ring sideroblasts

refractory cytopenia with multilineage dysplasia 
RCUD

refractory cytopenia with unilineage dysplasia

WHO

World Health Organization

WPSS

WHO Prognostic Scoring System 


\section{$1 \quad$ Einleitung}

Das myelodysplastische Syndrom (MDS) ist eine Erkrankung mit komplexen pathophysiologischen Vorgängen und einer mehrstufigen, interdisziplinären Diagnostik. Die Erforschung des MDS ist essentiell für eine adäquate Diagnosestellung und darauf aufbauende Therapieplanung. Im Folgenden werden die Hintergründe und die Zielsetzung der Arbeit erläutert. Die Einleitung befasst sich mit der Beschreibung der funktionellen Anatomie des Knochenmarks, gefolgt von Hintergründen über die Entstehung, Untersuchung und Behandlung des myelodysplastischen Syndroms. Besondere Aufmerksamkeit wird dem diagnostischen Arbeitsablauf, vor allem der Rolle von Pathologie und Zytogenetik sowie deren Einfluss auf Diagnose, Prognose und Therapieplanung, geschenkt. Von den Herausforderungen, die sich in der täglichen klinischen Praxis ergeben, wird die Fragestellung der Arbeit abgeleitet.

\subsection{Hämatopoiese und Knochenmark}

Die Hämatopoiese, die Neubildung von Blutzellen, ist ein notwendiger physiologischer Vorgang. Die Notwendigkeit zur Regeneration ergibt sich aus den natürlich begrenzten Lebenserwartungen der verschiedenen Blutbestandteile (Erythrozyten, Thrombozyten, Leukozyten) und ermöglicht ein konstantes Level dieser Zellen in der Peripherie. Der Ort der Blutbildung beim Erwachsenen ist das Knochenmark. Die Kenntnis der Physiologie und der regulären funktionellen Anatomie ist Grundlage für die Beurteilung von Gewebsproben mit Verdacht auf MDS, bei der eine Störung derselben vorliegt. Die folgenden Abschnitte führen in diese Thematik ein.

\subsubsection{Entwicklung der adulten Hämatopoiese}

Während der fetalen Entwicklung des menschlichen Organismus werden abhängig von Ort und Zeitpunkt drei hämatopoietische Phasen unterschieden: eine mesoblastische, eine hepatische und eine medulläre (Buchkley und Verfaillie 2011). Zuerst bilden sich in der dritten Schwangerschaftswoche Blutinseln in der Wand des Dottersacks. Ab der 6. Woche entwickeln sich im Extravasalraum der Leber hämatopoietische Zentren, ebenso in geringerem Ausmaß in der Milz. Mitte des zweiten Trimesters der Schwangerschaft beginnt die Blutbildung im Knochenmark, wo sie beim Erwachsenen ausschließlich stattfindet. Bei Versagen des Knochenmarks kann es jedoch auch später noch zu extramedullärer Blutbildung in Milz und Leber kommen. Physiologisch geht nach dem 4. Lebensjahr der Anteil der blutbildenden Skelettanteile von ursprünglich allen Knochen zunehmend zurück. Aus dem roten, 
hämatopoietisch aktiven Knochenmark entwickelt sich durch zunehmenden Fettgewebsanteil gelbes Knochenmark („Fettmark“). Im Erwachsenenalter findet die Blutbildung hauptsächlich in Sternum, Wirbelsäule, Rippen und Becken statt.

\subsubsection{Strukturbildende Elemente des Knochenmarks}

Das Knochenmark ist in die Substantia spongiosa der betreffenden Skelettanteile eingelagert. Die schwammartige Grundstruktur spongiöser Knochen wird durch die aus mineralisiertem Kollagen bestehenden Bälkchen (Trabekel) gebildet (Abbildung 1). Dazwischen liegen weite Räume, in denen die übrigen Gewebebestandteile lokalisiert sind.

Zentral in den Markräumen befindet sich ein System aus weitlumigen Sinusoiden. Es stellt, ähnlich zu Kapillaren in anderen Geweben, den Übergang zwischen dem arteriellen und venösen System dar. Diese spezielle Gefäßart ist dadurch gekennzeichnet, dass die sinusoidale Gefäßwand lumenseitig aus einem einreihigen, flachen Endothel, einer diskontinuierlichen Basalmembran und außen aus einer unvollständigen Hülle von Retikulum-Zellen besteht (Adventitia-Zellen) (Moonim und Porwit 2011). Die Fibroblasten spielen eine entscheidende Rolle in der Entstehung des speziellen Mikromilieus, in dem die Hämatopoiese stattfindet. Zum einen bilden sie durch Zellfortsätze und die Produktion einer extrazellulären Matrix ein maschenartiges dreidimensionales Gitter, auf denen die Blutzellvorstufen lokalisiert sind, zum anderen können sie deren Ausreifung durch Zytokine steuern (Bain et al. 2010) .

Funktionell stellen die Sinusoide ein geschlossenes System dar (Blut-KnochenmarksSchranke) (Moonim und Porwit 2011). Dies führt dazu, dass auswandernde Blutbestandteile sich transzellulär durch das Endothel bewegen müssen, um ins periphere Blut zu gelangen. Adhäsionsmoleküle tragen ebenfalls zum Verbleiben der Zellen im Knochenmark bei. Eine Fehlfunktion der Blut-Knochenmarks-Schranke mit Ausschwemmung unreifer Vorstufen ins periphere Blut ist Merkmal zahlreicher hämatologischer Erkrankungen, darunter auch des MDS.

Zum Stütz- und Bindegewebe des Knochenmarks gehören zudem Fettzellen, die manche Skelettanteile komplett auffüllen (,gelbes Mark`), aber auch im roten Mark mit steigendem Alter anteilig zunehmen. 

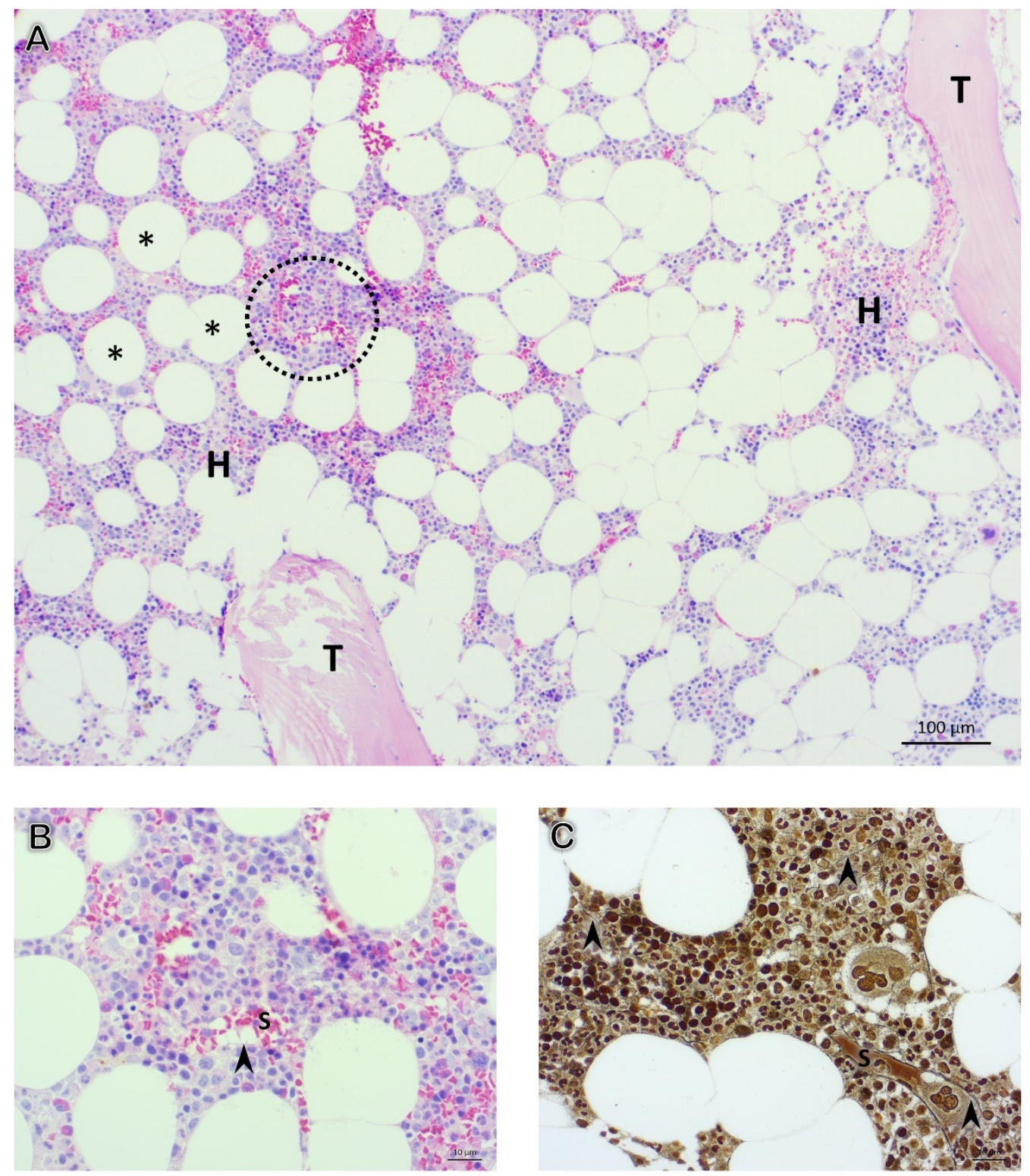

Abbildung 1: Strukturbildende Elemente des Knochenmarks

A: Übersichtsbild der Strukturen mit Knochentrabekeln $(\mathrm{T})$, hämatopoietische Zellen $(\mathrm{H})$, Fettzellen $\left(^{*}\right)$ und Sinusoiden (Kreis) [Kontrollpatient, Hämatoxylin-Eosin-Färbung, 10-fache Vergrößerung], B: Detailansicht eines Sinusoids: Endothelzellen ( $($ ) und mit Erythrozyten gefülltes Lumen (S) Kontrollpatient HämatoxylinEosin-Färbung, 40-fache Vergrößerung], C: Detaildarstellung der zarten, maschendrahtartigen extrazellulären Matrix (૪) [Kontrollpatient, Versilberung, 40-fache Vergrößerung], Quelle: eigene Präparate

\subsubsection{Hämatopoiese}

Im Extravasalraum zwischen der Spongiosa des Knochens und dem sinusoidalen Netzwerk liegen die unreifen und reifen Stufen der Hämatopoiese (Moonim und Porwit 2011). Die Entwicklung der Blutzellen ist hierarchisch organisiert. Den Ausgangspunkt stellt die hämatopoietische Stammzelle (HSZ, pluripotent) dar. Ihr vorgeschaltet ist nur noch die embryonale Stammzelle (totipotent), die in ihrer Entwicklung nicht auf eine Gewebeart festgelegt ist. Die HSZ zeichnet sich durch die Fähigkeiten der Selbsterneuerung und der Differenzierung aus. Über die genaue Einteilung der verschiedenen Blutzellreihen in die Hierarchie der 
Hämatopoiese wird aktuell noch diskutiert. Die folgenden Erläuterungen beziehen sich auf die Einteilung nach Seita und Weissman (2010), die auch in Abbildung 2 wiedergegeben ist. Im ersten Schritt entstehen zwei große Gruppen von multipotenten Progenitorzellen: die common myeloid progenitor (CMP)-Zellen sowie die common lymphoid progenitor (CLP)-Zellen. Die CLP-Zellen entwickeln sich zu T (Thymus)-, B (Bursa fabricii)- und NK (natural killer) -Zellen. Die T-Zellen wandern früh in den Thymus aus, B-Zellen verbleiben noch im Knochenmark. Ihre endgültige immunologische Ausreifung erfolgt allerdings in peripheren lymphatischen Organen, wie Lymphknoten, Milz oder Mukosa-assoziiertem lymphatischen Gewebe (MALT).

Im Gegensatz dazu erfolgt der größte Teil der myeloischen Ausreifung im Knochenmark. Die nächste Entwicklungsstufe nach dem CMP ist die GMP (Granulozyten/Monozyten Progenitor)-Zelle sowie die MEP (Megakaryozyten/Erythrozyten Progenitor)-Zelle. Aus der GMP entstehen neutrophile, eosinophile, basophile, dendritische sowie monozytäre Vorläuferstufen, die zu den entsprechenden Zellen des peripheren Blutes heranreifen. Aus der MEP-Zelle entwickeln sich die erythrozytäre Reihe sowie die Megakaryozyten.

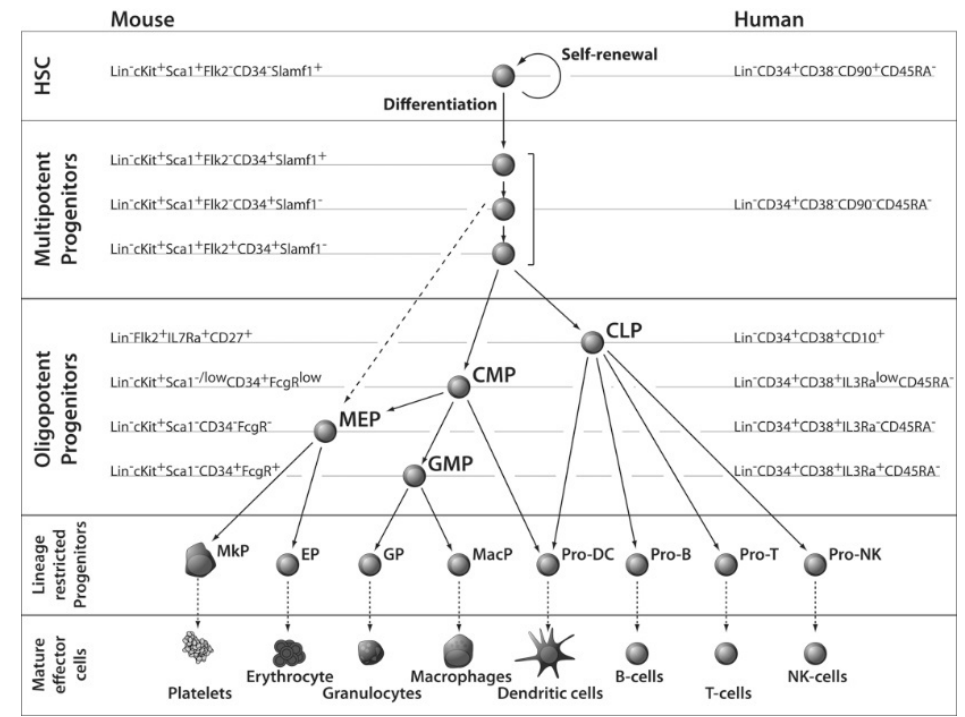

Abbildung 2: Modell der hierarchisch organisierten Hämatopoese

CLP: common lymphoid progenitor, CMP: common myeloid progenitor, DC: dendritic cell, EP: erythrocyte progenitor, GMP: granulocyte/macrophage progenitor, GP: granulocyte progenitor, HSC: hematopoietic stem cell, MacP: macrophage progenitor, MEP: megakaryocyte/erythrocyte progenitor, MkP: megakaryocyte progenitor, NK: natural killer, es sind noch verschiedene Immunmarker (Lin: linage marker) angeben, aus Seita und Weissman (2010) mit freundlicher Genehmigung von John Wiley \& Sons, Inc.

\subsubsection{Regulation}

Die Differenzierung der verschiedenen Blutbestandteile unterliegt der Regulation durch Botenstoffe. Zum einen werden Zytokine in dem Mikromilieu des Knochenmarks (Fibroblasten, Endothelzellen, etc.) gebildet, wie zum Bespiel der granulocyte-colony-stimulating factor (GCSF) oder Interleukin. Zum anderen kann diese Kontrolle durch Hormone aus 
knochenmarksfernen Organen erfolgen, beispielsweise mittels Erythropoietin oder Thrombopoietin aus Leber und Niere.

\subsubsection{Granulopoiese}

Die Entwicklungsstufen der Granulopoiese reichen von der GMP-Zelle bis zum ausgereiften neutrophilen Granulozyten. Im Knochenmark stellt sie die führende Zellreihe dar mit einem physiologischen Verhältnis zur Erythropoiese von 3:1 bis 4:1. Wie die Ausreifung der eosinophilen und basophilen Granulozyten in den hierarchischen Stammbaum der Hämatopoiese einzuordnen ist, konnte bislang nicht geklärt werden. Die erste morphologisch erkennbare Zelle der neutrophilen Differenzierung ist der Myeloblast, gefolgt von Promyleozyt, Myelozyt, Metamyelozyt sowie stab- und segmentkernigen Granulozyten.

Histologisch können diese verschiedenen Differenzierungsstufen zum Teil unterschieden werden (Moonim und Porwit 2011). Ein Myeloblast ist gekennzeichnet durch wenig Zytoplasma und einem großen Kern mit aufgelockertem Chromatin und einigen kleinen Nukleoli (Abbildung 3). Ein Promyelozyt hat nur noch einen Nukleolus, ist etwas größer und lagert im Zytoplasma Granula ein. Ein Myelozyt weist einen ovalen Kern mit kondensiertem Chromatin ohne Nukleoli und einen großen Zytoplasmaraum mit Granula auf. In der weiteren Ausreifung kondensiert das Chromatin stärker, wird zunächst stabkernig (Hufeisenform) und anschließend segmentkernig (mit mehreren durch dünne Chromatinfäden verbundenen Segmenten). In der mikroskopischen Übersicht liegen die frühen granulozytären Vorstufen saum- oder wallartig nahe der Knochentrabekel und den Gefäßen, während reifere Stufen sich in der Markraummitte befinden. 

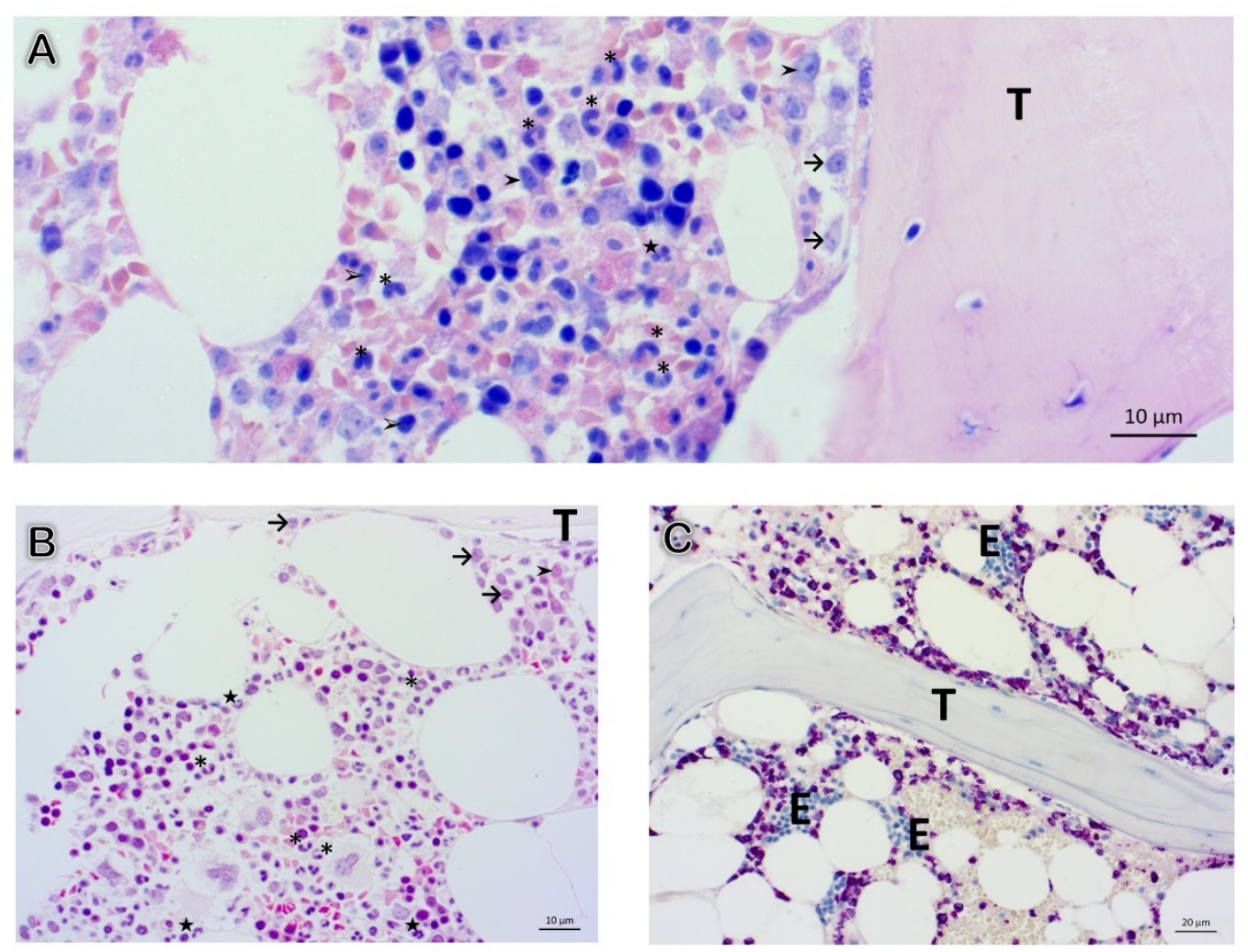

Abbildung 3: Normale Granulopoiese

A/B: Ausreifung der Granulozyten zur Markraummitte hin; peritrabekulär ( $\mathrm{T}$ ) hauptsächlich frühe Vorstufen, wie Myeloblasten (helles Chromatin, z. T. mehrere Nukleoli, wenig Cytoplasma: $\rightarrow$ ), Promyelozyten (dunkleres Chromatin, ein Nukleolus, Granula im Zytoplasma: >) und Myelozyten (kondensierter Kern, kein Nukleolus, großes Zytoplasma: ১). Die späteren Formen liegen eher in der Markraummitte, wie Metamyelozyten und stabkernige Granulozyten (sichel- oder hufeisenförmiger Kern, großes Zytoplasma: *) sowie segmentkernige Granulozyten (mehrere über dünne Filamente verbundene Chromatinsegmente: ^) [A: Giemsa-Färbung, B: Hämatoxylin-Eosin-Färbung, Kontrollpatient, 40-fache Vergrößerung], C: Übersicht; peritrabekuläre (T) Anordnung der Granulopoiese mit einem Verhältnis von Granulopoiese zu dem erythrozytären Nestern (E) von etwa 3:1 [C: Kontrollpatient, CAE-Färbung, 20-fache Vergrößerung]; Quelle: eigene Präparate

\subsubsection{Erythropoiese}

Die Erythropoiese beschreibt die Entwicklung eines Erythrozyten aus einer MEP-Zelle. Im Differenzierungsverlauf nehmen der intrazelluläre Organellengehalt sowie die Zellgröße ab, während die Menge an Hämoglobin zunimmt. Fünf Stufen werden unterschieden (E1-E5), die noch einen Kern enthalten. Nach dessen Abstoßung spricht man von einem Retikulozyten, weil noch netzartige (retikuläre) Zellorganellreste in seinem Zytoplasma darstellbar sind. Diese werden in der Milz abgebaut. Die Menge an Retikulozyten im peripheren Blut ist ein Marker für die Regenerationsleistung des Knochenmarks (bspw. mit Hilfe des Retikulozytenreproduktionsindex).

Im der mikroskopischen Ansicht ist die Erythropoiese in der Nähe der Sinusoiden in der Markraummitte angesiedelt (Moonim und Porwit 2011). Die Erythrozyten-Vorstufen bilden dabei Cluster oder Nester, die auch als Erythronen bezeichnet werden (Abbildung 4). In der Mitte dieser erythroblastischen Insel liegt ein Makrophage, der die abgespaltenen Zellreste 
phagozytiert (Chasis und Mohandas 2008). Histologisch ist dieser jedoch selten abzugrenzen. Ebenfalls wichtig ist die Interaktion zwischen den erythrozytären Vorstufen und den Makrophagen für die dreidimensionale Anordnung und die Steuerung der Differenzierung. Das erythroblast macrophage protein (Emp) vermittelt die Adhäsion (Hanspal et al. 1998). Stabilisierend wirkt zudem die Verbindung zwischen dem erythrozytären intercellular adhesion molecule-4 (ICAM-4) mit dem $\alpha$ V-Integrin der Makrophagen (Lee et al. 2006). Wird die letztere Verbindung blockiert, kommt es zu einer 70 \%igen Abnahme von erythrozytären Inseln sowie einem verminderten Ansprechen auf eine Erythropoetingabe (Mohandas und Chasis 2010). Histologisch fallen Erythronen vor allem durch die chromatindichten, homogen dunklen und basophilen runden Kerne der Normoblasten auf. Innerhalb der Erythrone lassen sich verschiedene Reifungsstufen erkennen (Moonim und Porwit 2011). Die Proerythroblasten zeichnen sich durch einen großen Zellkern und lockeres Chromatin aus. Im Laufe der Ausreifung zum Normoblasten kondensiert das Chromatin, der Kern wird kleiner und der Plasmasaum größer. Im Zytoplasma nimmt azidophiles Hämoglobin zu. Tendenziell befinden sich frühe Vorstufen eher zentral im Erythron (Chasis und Mohandas 2008). Eine Abgrenzung zu Lymphozyten kann anhand des homogener kondensierten Chromatins der erythropoietischen Vorläuferzellen erfolgen (Bain et al. 2010). 

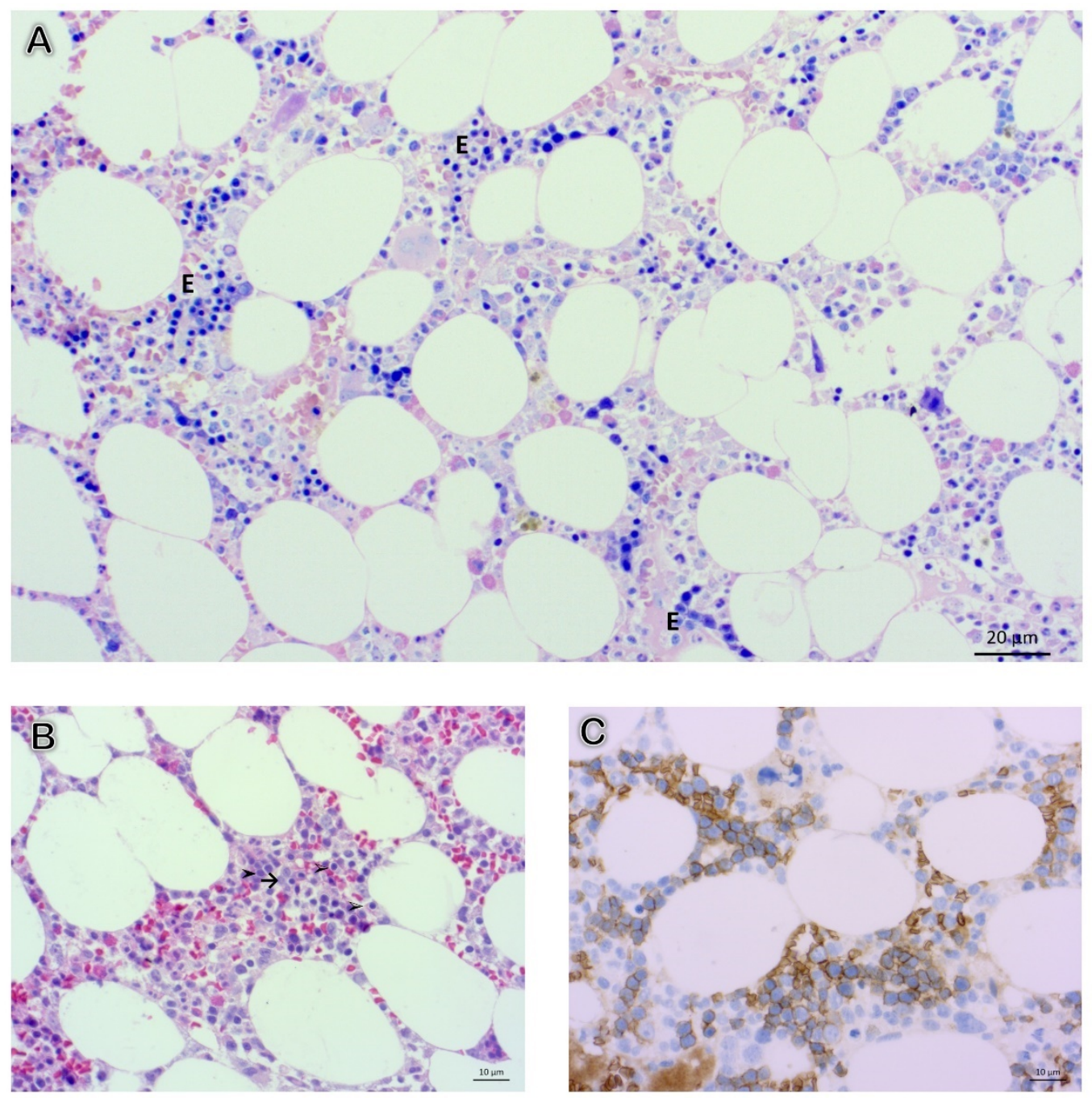

Abbildung 4: Normale Erythropoiese

A: Übersichtsbilder der Erythronen (E) [Kontrollpatient, Giemsa-Färbung 20-fache Vergrößerung]; B: Detailansicht der einzelnen zellulären Entwicklungsstufen; Proerythroblasten mit großem Kern, wenig kondensiertem Chromatin und wenig Zytoplasma $(\rightarrow)$, früher Normoblast mit stärker kondensiertem Chromatin ( $)$, später Normoblast mit stark kondensiertem Chromatin und fixationsbedingtem „Halo-Effekt“ ( $\triangleright$ ) [Kontrollpatient, HE-Färbung, 40-fache Vergrößerung], C: Detailansicht der Erythronen (E) mit verschieden großen Entwicklungsstufen [Kontrollpatient, Glycophorin-C-Immunhistochemie, 40-fache Vergrößerung]; Quelle: eigene Präparate

\subsubsection{Megakaryopoiese}

Aus der MEP-Zelle entwickeln sich ebenfalls die Megakaryozyten (Abbildung 5). Sie liegen einzeln und locker verteilt in der Markraummitte nahe an Sinusoiden, durch deren Endothel sie Zellausläufer (Pseudopodien), die bis in den Blutstrom reichen, bilden. Diese werden abgespalten und als Thrombozyten ins periphere Blut entlassen (Tavassoli und Aoki 1989). Eine Verlagerung der Megakaryozyten in die Nähe der Knochentrabekel ist Zeichen einer Atypie (Bain et al. 2010).

Histologisch zeichnen sich Megakaryozyten vor allem durch ihre Größe und Mehrkernigkeit aus. Dies ist auf den Mechanismus der Endoreplikation, also Chromosomenverdopplung ohne Kernteilung (Mitose), zurückzuführen (Deutsch und Tomer 2006). Hieraus ergibt sich 
eine auffällige Lappung (Lobulierung) des Zellkerns. Insgesamt ist das Chromatin aufgelockert. Die Megakaryozyten fallen zudem durch ein ausgedehntes Zytoplasma auf (Moonim und Porwit 2011). Ein weiteres morphologisches Phänomen ist die Emperipolese, bei der es zu einer Durchwanderung der Megakaryozyten durch andere Zellen kommt. Hierbei handelt es sich am ehesten um eine benigne Alterserscheinung ohne Krankheitswert. Unter der Verwendung immunhistochemischer Marker, wie CD61, sind auch frühere, histomorphologisch nicht zu identifizierende Formen abgrenzbar (Bain et al. 2010).
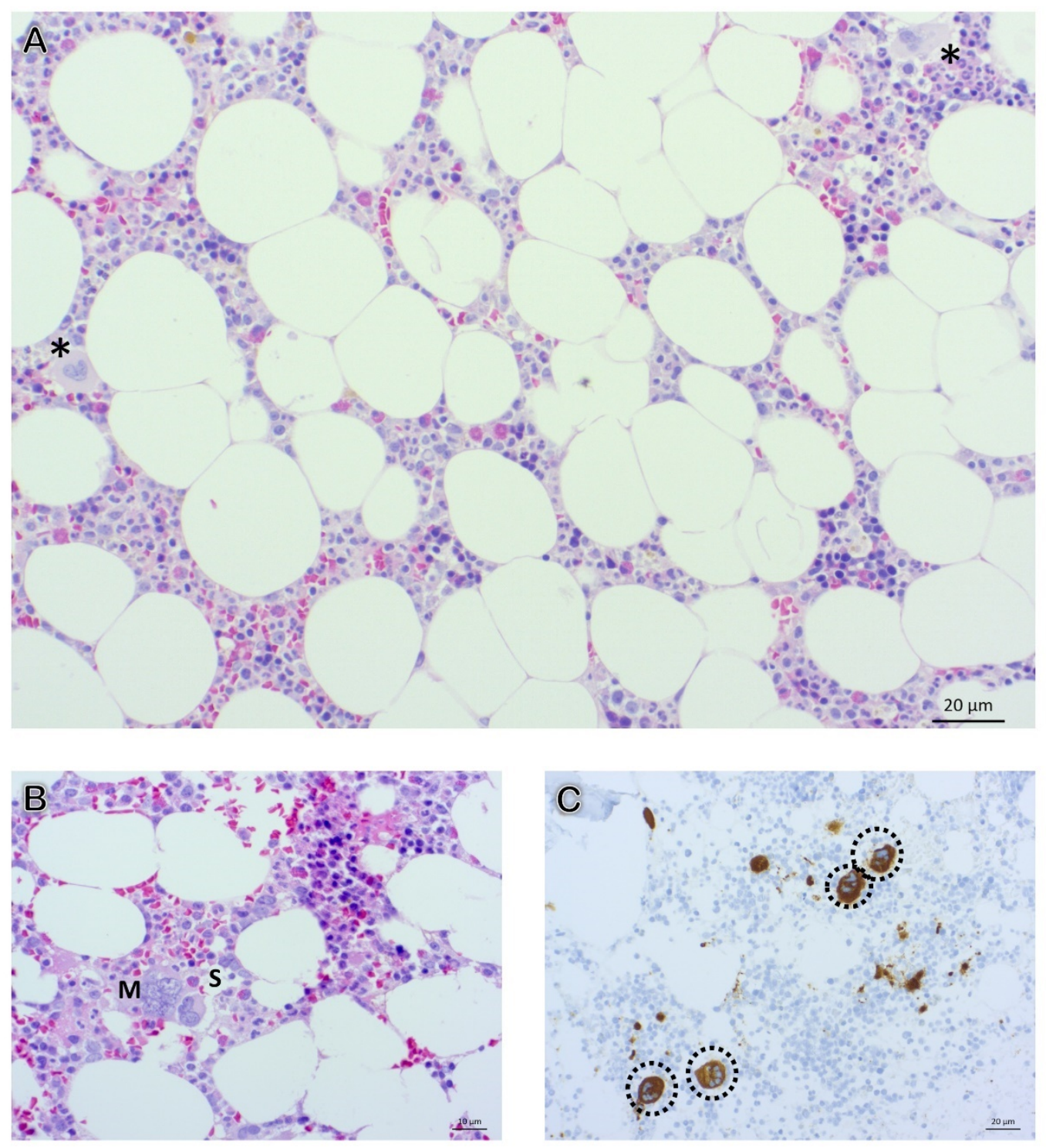

Abbildung 5: Normale Megakaryopoiese

A: Übersichtsansicht der vereinzelt liegenden Megakaryozyten (*) [Kontrollpatient, Hämatoxylin-Eosin-Färbung, 20-fache Vergrößerung], B: Detailansicht eines großen, mehrkernigen Megakaryozyten (M) in der Nähe eines Sinusoids (S) [Kontrollpatient, Hämatoxylin-Eosin-Färbung, 40-fache Vergrößerung] C: Übersichtsdarstellung der vereinzelt liegenden mehrkernigen Megakaryozyten (Kreise) [Kontrollpatient, CD61Immunhistochemie, 20-fache Vergrößerung]; Quelle: eigene Präparate 


\subsection{Myelodysplastisches Syndrom (MDS)}

Beim MDS ist die in Kapitel 1.1 beschriebene normale Architektur des Knochenmarks gestört. Die Bezeichnung „myelodysplastisch“ (aus dem Altgriechischen: „myelon“ = das Mark, ,dys" = Fehl- und ,plasis ${ }^{c}=$ die Form) trägt diesem Befund Rechnung. Eingeteilt wird MDS nach der WHO-Klassifikation hämatolymphatischer Tumoren, erschienen in der WHO Classification of Tumours (Swerdlow et al. 2008). Entscheidend sind neben einem Ausschluss anderer Ursachen vor allem die Ergebnisse aus dem peripheren Blut mittels Laborbestimmung (Zytopenie, Blastenanteil), der Knochenmarksbeurteilung in Aspirat und Biopsie (Dysplasie, Blastenanteil, Ringsideroblasten) sowie die Zytogenetik (Arber et al. 2016). Andere Kriterien (Transfusionsbedarf, Alter, Morbidität) sind entscheidend bei der Prognosebestimmung und Therapieentscheidung.

\subsubsection{Epidemiologie}

MDS ist eine vergleichsweise seltene onkologische Erkrankung, weist jedoch markante Merkmale in Bezug auf die Alters- und Geschlechtsverteilung auf. Die Inzidenzrate wird in neusten Analysen mit ca. 4/100.000 angegeben. Diesbezüglich zeigt sich eine Übereinstimmung zwischen Erhebungen in Deutschland und den USA (Ma et al. 2007; Neukirchen et al. 2011). Auffälligerweise ist MDS fast ausschließlich eine Erkrankung des älteren Menschen: $86 \%$ der Patienten mit MDS sind älter als 60 und nur $6 \%$ sind jünger als 50 Jahre (Ma et al. 2007). Wahrscheinlich handelt es sich bei diesen Inzidenzraten um konservative Berechnungen. Aufgrund der Alterung der Gesellschaft und der verbesserten Diagnostik ist mit einer Zunahme der Inzidenz in den nächsten Jahren zu rechnen (Ma 2012). Andere Schätzungen gehen davon aus, dass bereits heute die Inzidenz deutlich höher liegt, aufgrund unspezifischer Symptome in frühen Krankheitsstadien aber noch immer zu selten oder zu spät diagnostiziert wird (De Roos et al. 2010; Cogle et al. 2011; McQuilten et al. 2014). Nach Schätzungen von Cogle at al. (2011) könnte die Inzidenzrate in der Altersgruppe über 65 Jahre bei 75/100.000 liegen. Eine Steigerung der Inzidenz in der Zukunft führt zu vermehrtem Bedarf an diagnostischer Abklärung.

Abgesehen vom Alter beeinflusst auch das Geschlecht die Wahrscheinlichkeit des Auftretens von MDS. Männer sind signifikant häufiger betroffen als Frauen. Die altersangepasste Inzidenzrate liegt bei 4,5 pro 100.000 pro Jahr für Männer und bei 2,7 pro 100.000 pro Jahr für Frauen (Ma et al. 2007). 


\subsection{2 Ätiologie}

Hinsichtlich der Entstehung von MDS wurden verschiedene Faktoren identifiziert, die ein erhöhtes Auftreten der Erkrankung bedingen oder mit ihr assoziiert sind. Eine Ursache lässt sich nur bei einem geringen Anteil der Patienten (ca. 15 - $20 \%$ ) ausmachen, erlaubt aber meist eine differenziertere Aussage zur Prognose der Erkrankung (Adès et al. 2014). Zu unterscheiden sind seltene erbliche Ursachen, wie familiäres MDS oder angeborenes Knochenmarksversagen, von im Laufe des Lebens erworbenen Risikofaktoren, wie mutagene Exposition (Radiochemotherapie, Zigarettenrauch, Arbeits-/Umweltgifte), oder als Folgeerscheinung bestehender Erkrankungen (rheumatischer Formenkreis, aplastische Anämie, Adipositas).

Vererbbare Ursachen spielen insbesondere in der Abklärung von MDS im Kindesalter (ca. $5 \%$ aller Fälle) eine Rolle. Verschiedene Erkrankungen, die mit einem angeborenen Versagen des Knochenmarks einhergehen, sind in unterschiedlicher Stärke mit MDS assoziiert (Niemeyer und Baumann 2008). Hierzu zählt die Fanconi-Anämie (kumulative Inzidenz im Alter von 40 Jahren: 33 \% für akute myeloische Leukämie und MDS) (Kutler et al. 2003), die schwere kongenitale Neutropenie unter Therapie mit G-CSF (kumulatives Risiko nach 15 Jahren Therapie von $22 \%$ ) (Rosenberg et al. 2010) sowie weitere seltenere Erkrankungen, wie das Shwachman-Diamond-Syndrom, die Dyskeratosis congenita und die DiamondBlackfan-Anämie mit einem unterschiedlich erhöhten Risiko (Niemeyer und Baumann 2008). Direkt vererbte Myelodysplasien sind sehr selten und die genetischen Risikofaktoren noch ungenügend erforscht, sodass aus einer humangenetischen Abklärung nicht in jedem Fall eine Handlungshilfe abgeleitet werden kann (Holme et al. 2012).

Häufiger als erbliche lassen sich erworbene Ursachen für ein MDS ausmachen, wobei mutagene Substanzen von assoziierten Krankheiten zu unterscheiden sind. Im Vordergrund steht ein durch (Radio)-Chemotherapie ausgelöstes Therapie-assoziiertes MDS (t-MDS). Dies lässt sich bei ca. $15 \%$ der Patienten anamnestisch nachweisen und wird - auch im Rahmen der WHO-Klassifikation - als eigner Subtyp betrachtet und mit der Therapie-assoziierten AML (akute myeloische Leukämie) als Therapy-related myeloid neoplasms zusammengefasst (Arber et al. 2016). Alkylierende Substanzen (Cylcophosphamid, Melphalan) können diese späte Arzneimittelnebenwirkung verursachen. Der Krankheitsverlauf ist charakterisiert durch eine Latenz zwischen t-MDS und AML von 3 bis 10 Jahren, einer vor der AML auftretenden MDS-Phase sowie häufigen genetischen Aberrationen an den Chromosomen 5 und 7 (Godley und Larson 2008). Im Gegensatz hierzu zeigt das t-MDS nach Therapie mit Topoisomerease-II-Hemmern (beispielsweise Etoposid) einen aggressiveren Verlauf (Latenz unter 3 
Jahren, seltenere MDS-Phase) (Churpek und Larson 2013). Charakteristische Aberrationen sind Translokationen innerhalb der chromosomalen Banden 11q23 oder 21q22 (Olney et al. 2002). Neben Zytostatika erhöhen auch arbeits- und lebensstilabhängige Substanzen wie Benzol (Strom et al. 2008), Pestizide (Jin et al. 2014), Rauchen (Björk et al. 2000; Strom et al. 2005) sowie Übergewicht (Ma et al. 2009) das Risiko, an MDS zu erkranken.

Bestimmte Stammzellerkrankungen gehen mit einem erhöhten Risiko für MDS einher. Dieses Risiko besteht bei einer aplastischen Anämie (Progress-Rate in verschiedenen Studien 1,7 $\%$ bis $57 \%$ ) (Afable et al. 2011). Gleiches gilt für die paroxysmale nocturale Hämaturie (PNH) und MDS, wobei das für PNH typische defekte GPI-Ankerprotein bei ca. $20 \%$ der Patienten mit Niedrig-Risiko-MDS nachweisbar war (Sugimori et al. 2009). Diese Patienten zeigten einen milderen Verlauf und ein besonders gutes Ansprechen auf Immunsuppression (Ishiyama et al. 2003). Zusätzlich zu den assoziierten hämatologischen Erkrankungen beobachteten Wilson et al. (2014) ein leicht erhöhtes Risiko für das Auftreten von MDS bei vorbestehenden Autoimmunerkrankungen. Die genaue Relevanz dieses Zusammenhangs konnte noch nicht geklärt werden.

\subsubsection{Pathogenese und Pathophysiologie}

Wie es zu einer Transformation des normalen, gesunden Knochenmarks in ein myelodysplastisches Syndrom kommt, ist zum jetzigen Zeitpunkt noch nicht vollständig verstanden. In den letzten Jahren konnten jedoch verschiedene Mechanismen entdeckt werden, die bei der Pathogenese eine Rolle spielen. Bestimmte Veränderungen können bereits spezifischen histologischen Kranksheitssubtypen und -stadien zugeordnet werden. Als Teilaspekte der Genese von MDS werden im Folgenden die ineffektive Hämatopoese, Haploinsuffizienz, somatische Genmutationen, das Immunsystem und das Mikromilieu des Knochenmarks betrachtet. Besonders interessant sind zudem die Ursachen für die morphologischen Veränderungen des Knochenmarks bei MDS (Adès et al. 2014).

Das Konzept der ineffektiven Hämatopoiese steht schon seit langem im Zentrum des Verständnisses des MDS. Es geht davon aus, dass zu Beginn der Erkrankung die hämatopoetischen Stammzellen die Fähigkeit verlieren, sich zu normal differenzierten Tochterzellen weiterzuentwickeln und gleichzeitig vermehrt absterben (Apoptose) (Raza et al. 1995; Corey et al. 2007). Dies führt zum paradoxen Bild von Zytopenien im peripheren Blut bei einem gleichzeitig vollen, hyperplastischen Knochenmark, das aus ausreifenden, aber dysplastischen Zellen besteht, die häufig nicht effizient in das periphere Blut entlassen werden können. Ein Anzeichen für eine Krankheitsprogression mit schlechterer Prognose ist das vermehrte 
Vorkommen von Blasten. Der häufig beobachtete Übergang von MDS in eine akute myeloische Leukämie kann pathogenetisch dadurch erklärt werden, dass HSZ durch zusätzliche genetische Aberrationen resistenter gegen Apoptose werden und verstärkt proliferieren (Corey et al. 2007).

Haploinsuffizienz beschreibt den Verlust eines Allels und führt oft zu einer verminderten Gen-Expression (Adès et al. 2014). Sie kommt bei MDS in verschiedenen Formen vor, beispielsweise $\operatorname{del}(5 q), \operatorname{del}(7 q)$ oder $\operatorname{del}(20 q)$. Am besten verstanden ist die Relevanz der Haploinsuffizienz beim MDS mit del(5q). Ein Block in der Proliferation und Differenzierung von Erythrozyten entsteht hier durch Haploinsuffizienz der ribosomalen Untereinheit RPS14 (Ebert et al. 2008; Barlow et al. 2010) und bewirkt eine Reifungsstörung der Megakaryozyten und Thrombozytose durch die Haploinsuffizienz bestimmter Mikro-RNAs (Starczynowski et al. 2010). Diese Veränderungen resultieren in einem spezifischen morphologischen Äquivalent, der Verminderung der Erythropoiese und Megakaryozyten mit kleinen, nicht-lobulierten, runden Kernen (van den Berghe et al. 1974). Diese Erkenntnisse haben bereits Einfluss auf die Therapie. Der del(5q)-Subtyp zeigte ein verbessertes Ansprechen auf Lenalidomid, das supprimierend wirkt (Wei et al. 2009).

In ca. $50 \%$ der Fälle lässt sich eine im Karyogramm sichtbare chromosomale numerische oder strukturelle Aberration nachweisen. Bei MDS handelt es sich im Gegensatz zur AML diesbezüglich meist um eine Aneuploidie oder eine Deletion, wie beispielsweise das erwähnte 5q-Syndrom (Corey et al. 2007). Allerdings treten diese Veränderungen vermehrt nach einem länger bestehenden MDS auf, sodass eine vorbestehende genetische Instabilität aufgrund anderer genetischer Veränderungen anzunehmen ist. So konnte in den letzten Jahren durch molekulargenetische Methoden gezeigt werden, dass sich auch bei der anderen Hälfte der Patienten im Karyogramm nicht sichtbare Mutationen nachweisen lassen. In einer kleineren Anzahl der Fälle (ca. 15\%) betraf dies Transkriptionsfaktoren, Cohesine und Teile des Kinase-Signalweges. In den Fokus des Interesses gerückt sind vor allem Gene der epigenetischen Regulatoren und der Splicing-Faktoren. Sie waren in $70 \%$ der Fälle mutiert $(25 \%$ Splicing-Faktoren, $20 \%$ epigenetische Regulatoren, $25 \%$ beide) (Bejar und Steensma 2014). Epigenetische Regulatoren kontrollieren die Aktivität des entsprechenden Gens durch Methylierung, Hydroxymethylierung oder kovalente Bindungen zwischen Histonen. Sie treten häufig in Kombination miteinander auf, finden sich insbesondere bei Patienten mit Blastenvermehrung und werden auch bei anderen hämatologischen Tumoren beobachtet. Den Regulatoren wird deshalb pathogenetisch vor allem eine Rolle bei der Progression eines MDS zugeschrieben (Jiang et al. 2009; Adès et al. 2014). Außerdem bieten sie ein Erklärungsmodell für die Wirksamkeit von hypomethylierenden Substanzen (Azacitidine, Decitabine) in der 
Therapie von MDS. Im Gegensatz dazu sind Mutationen in einem Splicing-Faktor-Gen sehr charakteristisch für MDS. Pro Patient trat jeweils nur eine Mutation in dieser Gruppe auf und das zu einem frühen Zeitpunkt der Erkrankung (Yoshida et al. 2011; Mian et al. 2013). Walther et al. haben die genetischen Charakteristika eines Progresses von MDS zu AML untersucht. Sie konnten feststellen, dass unabhängig von der Blastenzahl sich ein Großteil der Zellen im Knochenmark klonal aus einer Ursprungszelle mit MDS entwickelt hatte. Die Patienten mit AML besaßen verschieden große Tochterklone aus diesem Urspungsklon, wobei letzterer häufig verdrängt wurde (Walter et al. 2012).

Neben den genetischen Veränderungen der HSZ muss auch die Rolle externer Faktoren, wie des umgebenden Stromas, bei der Entstehung und des Fortschreitens von MDS betrachtet werden. So konnte beispielsweise gezeigt werden, dass die Deletion des Dicer1-Gens in Mäuse-Osteoprogenitorzellen morphologisch zu einer Dysplasie hämatopoietischer Zellen und einem Fortschreiten in eine AML führte (Raaijmakers et al. 2010). Des Weiteren verloren die Stromazellen der Patienten mit MDS zunehmend Adhäsionsmoleküle (Aanei et al. 2012). Dies legt nahe, dass auch die Stammzellnische als Mikromilieu aus Bindegewebszellen und extrazellulärer Matrix die Pathogenese von MDS beeinflusst.

Ein ebenfalls externer Faktor in der MDS-Pathogenese ist die Deregulation des Immunsystems. Eine zentrale Rolle nehmen die regulatorischen $\mathrm{CD}^{+}{ }^{+} \mathrm{T}-$ Zellen ein. Physiologisch haben sie die Aufgabe, eine überschießende immunologische Reaktion (Autoimmunität) zu verhindern. Dies kann dazu führen, dass die gegen eine Neoplasie gerichtete Immunreaktion abgemindert wird (Sakaguchi et al. 2010). Für das myelodysplastische Syndrom konnte gezeigt werden, dass eine erhöhte Anzahl von $\mathrm{CD}^{+}{ }^{+}$T-Zellen mit verschiedenen negativen Prognosefaktoren assoziiert ist (Kordasti et al. 2007). Darüber hinaus konnte eine Kinetik der regulatorischen T-Zellen für unterschiedliche Stadien der Erkrankung festgestellt werden: In frühen Stadien ist die Aktivität und das Einwandern ins Knochenmark im Vergleich zur Kontrollpopulation herabgesetzt, was für einen Autoimmunität begünstigenden Status spricht. Der Progress der Erkrankung ist mit einer erhöhten Anzahl von regulatorischen $\mathrm{CD}^{+}$T-Zellen assoziiert, was die Rolle der Immunsuppression in diesem Stadium unterstreicht. Beim Ansprechen der Patienten auf die Therapie fällt die Anzahl wieder ab (Kotsianidis et al. 2009). Eine weiterer Hinweis auf eine immunologisch mitbedingte Pathogenese nahelegt ist die Beobachtung, dass manche Patienten von einer immunsuppressiven Therapie mit Anti-Thymozyten-Globulin und Cyclosporin profitieren (Passweg et al. 2011).

Wenn man die verschiedenen ursächlichen Faktoren in der Entstehung von MDS zusammenfassend betrachtet, lässt sich feststellen, dass diese zum jetzigen Zeitpunkt nicht 
hinreichend voneinander zu trennen sind und kein eindeutiger pathophysiologischer Ablauf zu erkennen ist. Vielmehr steht eine Vielzahl von möglichen Einflussfaktoren nebeneinander. Es lässt sich meist nicht eindeutig sagen, ob die herausgearbeiteten Erkenntnisse Ursache oder Konsequenz der MDS-Entstehung sind. Auf dem kleinsten Nenner gebracht, ist der aktuelle Erkenntnisstand, dass verschiedene ätiologische Faktoren, wie genetische Veranlagung, Alter und Noxen (Umweltgifte, Radiotherapie, Medikamente), zu einer Änderung der genetischen Ausstattung der hämatopoietischen Stammzelle (somatische Mutationen, epigenetische Regulation, Haploinsuffizienz) führen. Diese Faktoren könnten aber auch indirekt über einen Einfluss auf die Stammzellnische oder das Immunsystems ein MDS verursachen. Am Ende resultiert eine myelodysplastische Stammzelle, die sich nicht mehr differenziert und vermehrt untergeht (ineffektive Hämatopoiese). Im weiteren Verlauf kommt es zu zusätzlichen Mutationen, die einen Übergang in eine akute myeloische Leukämie kennzeichnen (Adès et al. 2014). Diese pathophysiologischen Überlegungen bilden die Grundlage für die Befundung einer gestörten Knochenmarksarchitektur.

\subsubsection{Klinik}

Die Symptome, mit denen MDS-Patienten erstmalig vorstellig werden, sind unspezifisch, lassen sich jedoch zu einem gewissen Maß auf die betroffenen Zellreihen zurückführen. Eine Anämie bedingt Müdigkeit, verminderte Belastbarkeit und Konzentrationsmangel, eine Neutropenie verursacht Infektionen und eine Thrombozytopenie führt zu Blutungsneigung (Foran und Shammo 2012). In einer Vielzahl der Fälle mit Niedrig-Risiko-MDS wird eine Zytopenie zufällig bei Routineuntersuchungen entdeckt. Auch die bereits besprochenen epidemiologischen und ätiologischen Faktoren, wie hohes Alter oder Exposition gegenüber mutagenen Substanzen, können anamnestisch den Verdacht auf ein myelodysplastisches Syndrom lenken.

\subsubsection{Untersuchungsverfahren}

Grundsätzlich kommen bei der Diagnose des MDS zahlreiche Verfahren zur Anwendung. Sowohl verschiedene medizinische Disziplinen (Hämato-Onkologie, Pathologie, Labormedizin, Humangenetik) als auch Methoden (Blutausstrich, Zytologie, Histologie, Laborbestimmungen, Zytogenetik) sind Teile eines sich ergänzenden, mehrstufigen Prozesses. Zudem ist es wichtig, zu unterscheiden, ob mit dem angewendeten Untersuchungsverfahren eine (differential-) diagnostische - liegt ein MDS vor? - oder prognostische - liegt ein erhöhtes Risiko für Komplikationen oder Progress vor? - Aussage gemacht werden soll. In manchen Fällen lassen sich diese Gesichtspunkte (Diagnose versus Prognose) nicht genau voneinander 
trennen. So ist der Nachweis einer 5q-Deletion diagnostisch ein eigener Subtyp nach WHOKlassifikation und prognostisch als günstig zu bewerten. Die vorliegende Dissertation befasst sich mit dem Stellenwert der pathologischen Untersuchung einer Knochenmarksbiopsie (Histologie) in diesem Prozess.

Im Laborbefund der Patienten stehen in den meisten Fällen Zytopenien im Vordergrund. Zum Zeitpunkt der Diagnose besteht bei ca. $80 \%$ der Patienten eine Anämie, bei $40 \%$ eine Neutropenie und bei 30-45\% eine Thrombozytopenie (Steensma und Bennett 2006; Bryan et al. 2010). Die Anämie ist typischerweise makrozytär oder normozytär und nur in seltenen Fällen mikrozytär (Foran und Shammo 2012). In den meisten Fällen besteht eine Neutropenie oder Thrombozytopenie in Kombination mit einer Anämie als Bizytopenie. Auch eine Thrombozytose ist ein möglicher Befund bei MDS und kommt bei ca. $8 \%$ der Patienten zu Beginn der Erkrankung vor (Kodali et al. 2007). Unklare Zytopenien gehören zu den mit Abstand häufigsten Indikationen für eine Knochenmarkspunktion. Der Blutausstrich erlaubt einen schnellen, wenig invasiven Einblick in qualitative und quantitative Veränderungen des peripheren Blutes und lässt zum Beispiel hypolobulierte oder hypogranulierte Nuklei der Neutrophilen oder Anisozytose (Größenvariabilität), Poikilozytose (Formvariabilität) und Makrozytose der Erythrozyten erkennen (Bain 2005). Für die Einteilung des MDS in die verschiedenen Subtypen gemäß WHO-Klassifikation ist insbesondere der prozentuale Anteil von Blasten im Knochenmark und peripheren Blut entscheidend (Vardiman et al. 2009). Bei einer Knochenmarksaspiration wird zum einen Material für die morphologische Begutachtung, zum anderen für genetische Analysen gewonnen. Erstere ermöglicht die Ermittlung von Dysplasiezeichen der einzelnen Zellen und des Blastenanteils (Garcia-Manero 2014). Die Aspirationsprobe dient der Analyse einzelner Zellen des Knochenmarks, während die Biopsie für die Beurteilung des gesamten Gewebes geeignet ist. Insbesondere gibt letztere Aufschluss über die Zellularität, quantitative Verschiebungen zwischen den einzelnen Zellreihen, Architekturstörungen, Blastenanteil und Veränderungen der extrazellulären Matrix (siehe auch Kapitel 2.5). Sie gilt als besonders geeignet, bestimmte Unterformen, wie hypoplastisches MDS oder MDS mit Fibrose, zu erkennen (Della Porta et al. 2009; Garcia-Manero 2014). Des Weiteren hilft die Biopsie bei der Abgrenzung zu anderen hämatologischen Erkrankungen, wie primäre AML, aplastische Anämie oder myeloproliferatives Syndrom (Adès et al. 2014). Die Zytogenetik ist ein weiterer fester Bestandteil einer Abklärung von MDS, der in den letzten 20 Jahren zunehmend an diagnostischer und prognostischer Bedeutung gewonnen hat (siehe auch Kapital 2.3). In ca. 50 \% der Fälle lassen sich bei einer Biopsie aus dem Knochenmark im Karyogramm sichtbare Veränderungen nachweisen (Haase et al. 2007; Pozdnyakova et al. 2008; Greenberg et al. 2012). Als weitere genetische Untersuchung 
konnte die Sequenzierung von Genabschnitten ebenfalls bei der Prognoseabschätzung in ca. der Hälfte aller Patienten helfen. Dies gilt auch in einer Gruppe mit unauffälliger zytogenetischer Untersuchung (Bejar et al. 2011). Der Einsatz dieser Methode ist bislang noch nicht Standard in der Breitenversorgung, steht aber absehbar bevor. Für ein tiefergehendes Verständnis der Entstehung des MDS wie im Falle der oben erwähnten Rolle der Splicing-Faktoren oder der Genmethylierung, oder auch eine verbesserte Therapiestratifizierung der Patienten ist die Methode unerlässlich. Mit dem sogenannten Next Generation Sequencing (NGS) können bei 80-90\% der Patienten mit MDS Genmutationen in Genen nachgewiesen, die sehr häufig eine Funktion im Chromatinremodelling und der epigenetischen Regulation spielen (z.B. SF3B1, TET2, SRSF2, ASXL1, DNMT3A, RUNX1, U2AF1, TP53, EZH2). Wichtig ist hierbei, dass derartige Mutationen als sog. "clonal hematopoiesis of indeterminate potential (CHIP)" auch bei älteren Personen ohne MDS vorkommen und der alleinige Nachweis nicht die Diagnose eines MDS rechtfertigt, wobei die Patienten ein erhöhtes Risiko aufweisen, im Verlauf ein MDS zu entwickeln (Haferlach et al. 2014; Jaiswal et al. 2014; Steensma et al. 2015).

Verschiedene Verfahren werden in der Diagnostik des MDS in einem nebeneinander existierenden und sich ergänzenden Ansatz verwendet. Sie eignen sich dazu, die Verdachtsdiagnose $z u$ erhärten und zu bestätigen, bestimmte Subtypen zu identifizieren, die Prognose abzuschätzen und eine Therapie auszuwählen.

\subsubsection{Klassifikationen}

Um die Heterogenität des MDS besser abzubilden zu können, wurden mehrere Klassifikationen und Scores entwickelt. Unterscheiden lassen sich zwei große Gruppen: zum einen die hauptsächlich morphologischen Klassifikationen bezüglich der Diagnose eines MDS (FAB, WHO) und zum anderen die aus unterschiedlichen Kriterien zusammengesetzten Klassifikationen für die Abschätzung der Prognose und zur Therapiestratifizierung (IPSS, WPSS).

\subsubsection{Morphologische Klassifikationssysteme zur Bestimmung der Diagnose}

Ein Bericht über einen Patienten mit „Leukanämie“, also einer aus einer Anämie hervorgehenden Leukämie, wurde wahrscheinlich erstmals von Leube im Jahr 1900 in der Berliner Klinische Wochenschrift veröffentlicht (Nimer 2008). Block et al. (1953) beschreiben in einem Kollektiv von 12 Patienten, dass diese klinisch durch Zytopenien gekennzeichnet waren sowie im Knochenmark Leukämie-ähnliche Veränderungen aufwiesen, weswegen die Erkrankung als „Präleukämie“ bezeichnet wurde (eng.: prelenkemia oder prelenkemic phase). Im Verlauf fiel auf, dass viele Patienten mit prä-leukämischen Veränderungen im Knochenmark 
nicht an einem Progress, sondern den Folgen der Zytopenie verstarben. Für diese Erkrankung wurde erstmals von der French-American-British (FAB)-Arbeitsgruppe in 1976 der Begriff der „Myelodysplasie“ geprägt und das Krankheitsbild in die beiden damals bekannten Kategorien refractory anemia with excess blasts (RAEB) und chronic myelomonocytic lenkemia (CMML) eingeteilt (Bennett et al. 1976). In der Aktualisierung der FAB-Klassifikation wurde das myelodysplastische Syndrom später anhand morphologischer Kriterien in fünf Kategorien eingeteilt (Bennett et al. 1982), darunter die refractory anemia (RA) und refractory anemia with ring sideroblasts (RARS) als Entitäten ohne Blastenvermehrung. Des Weiteren wurden bei Patienten mit Blastenvermehrung die refractory anemia with excess blasts (RAEB) und die refractory anemia with excess blasts in transformation (RAEB-T) anhand der Blasten im Knochenmark (5 $20 \%$ vs. $21-30 \%)$ und im peripheren Blut $(1-4 \%$ vs. $\geq 5 \%)$ unterschieden. Mit der WHO-Klassifikation myeloider Neoplasien und akuter Leukämie von 2001 und ihren Revisionen von 2008 sowie 2016 wurden einige Änderungen vollzogen (Jaffe 2001; Swerdlow 2008; Arber et al. 2016). Die CMML wurde der Gruppe myeloproliferativer/myelodysplastischer Neoplasien (MDS/MPS) zugeordnet. Die vormalig zu RAEB-T gehörenden Patienten mit Blasten $>20 \%$ werden heute als AML klassifiziert und die RAEB-Gruppe in zwei Subtypen unterteilt (RAEB-1 und RAEB-2). Eingeführt wurde ebenfalls die Kategorie der refractory cytopenia with multilineage dysplasia (RCMD) für Patienten mit dysplastischen Veränderungen in zwei oder mehr Reihen. Die Gruppe der Patienten mit dysplastischen Veränderungen in nur einer Zellreihe werden in die RCUD-Gruppe (refractory cytopenia with unilineage dysplasia) eingeordnet, wobei hier auch nach der betroffenen Zelllinie unterschieden werden kann. Ebenfalls neu eingeführt wurde das MDS mit del(5q), das sich allein durch die Zytogenetik bestimmen lässt. Zudem können für MDS typische genetische Veränderungen mit nicht ausreichenden morphologischen Kriterien als MDS-unklassifizierbar (MDS-U) bezeichnet werden. Mit dem Update der Klassifikation im Jahr 2016 wurden hauptsächlich semantische Veränderungen vorgenommen (Arber et al. 2016). So wurde der Term „refraktäre Anämie/Zytopenie“ verlassen und stattdessen der Denominator „MDS mit ..." verwendet. Aus refractory cytopenia with unilineage dysplasia (RCUD) wurde so MDS with single lineage dysplasia (MDS-SLD), aus RCMD wurde MDS with multilineage dysplasia (MDS-MLD) und aus RAEB wurde MDS with excess of blasts (MDS-EB), das in Typ 1 und Typ 2 unterteilt wird. Die Anwendung dieser Kategorien bei der Bewertung der histologischen Biopsate im Rahmen der vorliegenden Dissertation wird Kapitel 2.6.7 besprochen. 
1.2.6.2 Multivariate Klassifikationssysteme zur Bestimmung der Prognose

Die WHO-Klassifikation ist geeignet, morphologische Mindestkriterien für die Diagnosestellung zu liefern und MDS von anderen hämatologischen Erkrankungen abzugrenzen. Jedoch wurden Limitationen beschrieben, den Verlauf der Erkrankung vorherzusagen. Verschiedene Prognose-Scores haben sich herausgebildet, von denen das WHO Prognostic Scoring System (WPSS) und das International Prognostic Scoring System (IPSS) am gebräuchlichsten sind.

Das 1997 eingeführte IPSS (Greenberg et al. 1997) betrachtet die Kriterien Knochenmarksblasten-Anteil, zytogenetische Risikogruppe und Zytopenien für die jeweils eine Punktzahl vergeben wird (Tabelle 1). Diese Kriterien erlauben nach einer Multivarianzanalyse die beste Aussage über die Prognose. Aus dem sich ergebenen Score lässt sich der Patient in eine von vier Risikogruppen (low, intermediate-1, intermediate-2 und bigh) einsortieren.

Tabelle 1: Kriterien des IPSS

\begin{tabular}{lccccc}
\hline Punkte & $\mathbf{0}$ & $\mathbf{0 , 5}$ & $\mathbf{1}$ & $\mathbf{1 , 5}$ & $\mathbf{2}$ \\
\hline Blastenanteil $(\%)$ & $0-4$ & $5-10$ & - & $11-20$ & $21-29$ \\
Zytopenien & $0-1$ & $2-3$ & - & - & - \\
Zytogenetische Risikogruppe & low & intermed. & high & - & - \\
\hline
\end{tabular}

Modifiziert nach: Greenberg et al. (1997), intermed. = intermediate

Im Rahmen einer Revision wurde dem IPSS-R keine neue Variable hinzugefügt, sondern die verschiedenen Kriterien aktualisiert und zum Teil in differenziertere Untergruppen sortiert (Greenberg et al. 2012) (Tabelle 2). Das IPSS hat in bestimmten Bereichen die Prognose nicht bestmöglich abgebildet. Dies wurde unter anderem durch folgende Änderungen aufgegriffen: Einteilung der Zytopenien nach Schweregrad, Hinzufügen neuer zytogenetischer Aberrationen (Solé et al. 2005; Haase et al. 2007; Pozdnyakova et al. 2008; Schanz et al. 2012) sowie Angleichen des Effekts der Hoch-Risiko-Zytogenetik und des Blastenanteils auf den Gesamtscore (Schanz et al. 2011). Die Addition der Punkte bildet einen Score, der nach dem IPSS-R in fünf Risikogruppen unterteilt. Der IPSS-R wurde als signifikantes prädiktives Tool für die Bestimmung des Gesamtüberlebens sowie des leukämiefreien Überlebens von Patienten eines anderen, unabhängigen Kollektivs validiert (Voso et al. 2013). 
Tabelle 2: Kriterien des IPSS-R

\begin{tabular}{lccccccc}
\hline Punkte & $\mathbf{0}$ & $\mathbf{0 , 5}$ & $\mathbf{1}$ & $\mathbf{1 , 5}$ & $\mathbf{2}$ & $\mathbf{3}$ & $\mathbf{4}$ \\
\hline Zytogenetisches Risiko & $\begin{array}{c}\text { sehr } \\
\text { günstig }\end{array}$ & - & günstig & - & intermediär & ungünstig & $\begin{array}{c}\text { sehr un- } \\
\text { günstig }\end{array}$ \\
Blastenanteil $(\%)$ & $\leq 2$ & - & $>2-<5$ & - & $5-10$ & $>10$ & - \\
Hämoglobin $(\mathrm{g} / \mathrm{dl})$ & $>10$ & - & $8-10$ & $<8$ & - & - & - \\
Plättchen $\left(* 10^{9} / \mathrm{l}\right)$ & $>100$ & $50-100$ & $<50$ & - & - & - & - \\
Neutrophile $\left(* 10^{9} / \mathrm{l}\right)$ & $>0,8$ & $<0,8$ & - & - & - & - & - \\
\hline
\end{tabular}

Modifiziert nach: Greenberg et al. (2012)

Das WHO prognostic scoring system (WPSS) stützt sich auf die Einteilung in die morphologischen WHO-Subtypen, Zytogenetik und Transfusionsbedarf, um eine Risikostratifizierung der Patienten vorzunehmen (Malcovati et al. 2007). Der Score wurde so konzipiert, dass er zu verschiedenen Zeitpunkten im Verlauf der Erkrankung angewendet werden kann. Die Einbeziehung des Transfusionsbedarfs berücksichtig einen relevanten klinischen Risikofaktor (Malcovati et al. 2005). Der WPSS unterscheidet fünf verschiedene Risikogruppen.

\subsubsection{Therapie}

Die Heterogenität des myelodysplastischen Syndroms zeigt sich auch in den verwendeten Therapieoptionen. Grundsätzlich erfolgt die Auswahl der geeigneten Behandlung auf Grundlage der Risikogruppe (bestimmt mittels IPSS-R, WPSS; Kapitel 1.2.6.2) und der individuellen Patientenfaktoren (Alter, Gesundheitszustand).

Patienten aus der low- und intermediate-1-Risko-Gruppe des IPSS werden supportiv (Bluttransfusionen, Eisenchelatoren, Wachstumsfaktoren) und/oder mit hypomethlylierenden Substanzen (Azacitidine, Decitabine) behandelt. Wenn del(5q) alleine oder in Kombination mit anderen Aberrationen vorliegt, wird zuerst Lenalidomid eingesetzt.

Für Patienten aus der intermediate-2- und high-Risiko-Gruppe des IPPS sollte zu Beginn eine Stammzelltransplantation als einzig mögliche kurative Option erwogen werden. Dies ist abhängig vom Alter und gesundheitlichen Zustand des Patienten sowie von der Verfügbarkeit eines geeigneten Spenders. Entscheidet man sich gegen eine Transplantation, so verbleiben als Therapieoptionen hypomethylierende Substanzen oder eine angepasste AML-Therapie (Lyons 2012). 


\subsection{Fragestellung}

MDS ist eine komplexe, heterogene Erkrankung, deren genaue pathogenetische Entstehung weiterhin unklar bleibt. Ebenfalls stellen Diagnostik, Prognosebestimmung und Therapieplanung in vielen Fällen eine Herausforderung dar. Die histopathologische Beurteilung einer Biopsie des Knochenmarks ist in der interdisziplinären diagnostischen und prognostischen Analyse ein Teilaspekt. Die Abklärung unklarer Zytopenien zählt zu den häufigsten Fragestellungen bei Knochenmarkspunktion. Erschwert wird die Beurteilung durch technische Probleme (kleine Proben, fehlende klinische Daten) und die Tatsache, dass Patienten dieser Altersgruppe häufig multimorbide sind (Niereninsuffizienz, Atemwegserkrankungen etc.) und häufig potentiell knochenmarksschädigende Medikamente einnehmen - Faktoren, die zu morphologischen Veränderungen führen können, die mit denen des MDS überlappen. Die WHO-Klassifikation als Richtlinie mit minimalen Kriterien zur Diagnosestellung ist hauptsächlich auf der Zytomorphologie aufgebaut, auch wenn einige histologische Eigenschaften bei Patienten mit MDS (Fibrose, Hypozellularität) bereits ausführlich beschrieben wurden. Der Zusammenhang zwischen den Untersuchungsverfahren (Morphologie und Genetik) und den klinischen Fragestellungen (Diagnose und Prognose) ist in Abbildung 6 dargestellt (schwarze Pfeile). Wenig beschrieben ist der Zusammenhang zwischen histologischen Veränderungen und der Zytogenetik (roter Pfeil). Im klinischen Alltag ist der Pathologe immer wieder zur Mitbeurteilung von Patienten mit Verdacht auf oder bekanntem MDS gefragt, auch zu einem Zeitpunkt, an dem noch keine zytologischen oder genetischen Untersuchungsbefunde vorliegen. Aus diesem Kontext ergeben sich die Fragestellungen der vorliegenden Dissertation.

1) Inwiefern ist die WHO-Klassifikation für die histologische Diagnostik brauchbar?

2) Gibt es einen Zusammenhang zwischen histomorphologischen Kriterien in der Knochenmarksbiospie und dem genetischen Risiko laut IPSS-R?

3) Falls ein Zusammenhang (siehe 2) besteht, wie zutreffend lassen sich anhand einzelner histomorphologischer Kriterien Aussagen über das genetische Risiko laut IPSS-R machen?

4) Welche anderen Zusammenhänge gibt es zwischen Histomorphologie, Genetik, Labordaten und klinischen Informationen? 


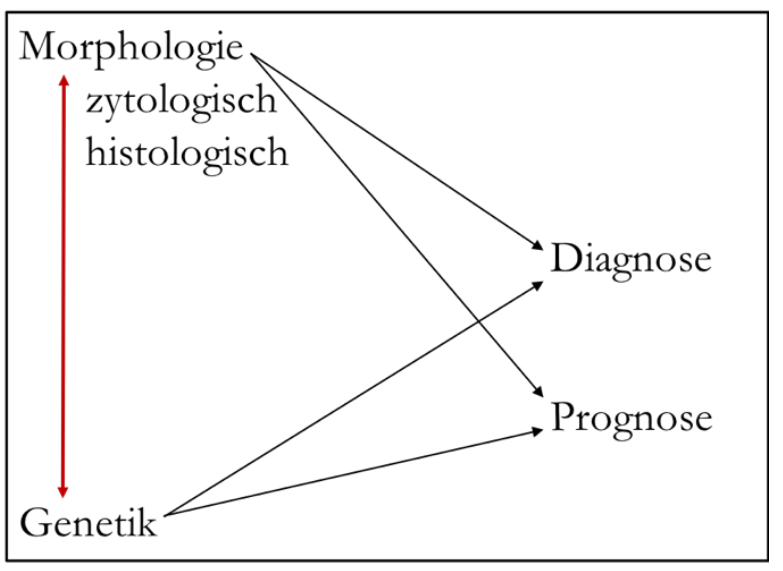

Abbildung 6: Zusammenhang der Untersuchungsmethoden bei MDS

Legende: Diese Abbildung zeigt die verschiedenen Untersuchungsmethoden und ihre Aussagekraft bei MDS. Während sich eine Vielzahl von Studien mit der diagnostischen und prognostischen Aussagekraft der Genetik und Morphologie (schwarze Pfeile) beschäftigt, liegt der Schwerpunkt der vorliegenden Dissertation auf der Frage, inwiefern sich eine Hochrisiko-Zytogenetik anhand der Morphologie voraussagen lässt; Quelle: eigenes Diagramm 


\section{$2 \quad$ Material und Methoden}

\subsection{Patientendaten}

Die Patientendaten wurden mit Hilfe der in der Klinikdatenbank (iXserv, ixmid Software

Technologie GmbH, Köln) hinterlegten Befunde (Pathologie, Labormedizin, Zytogenetik, Arztbriefe) erhoben und in bestimmten Fällen kategorisiert. Die Methodik der Datenerhebung wurde von der Ethikkommission der Medizinischen Fakultät der Georg-August-Universität Göttingen genehmigt (Antragsnummer: DOK_129_2013).

\subsubsection{Ein- und Ausschlusskriterien}

Die Patienten der Studie wurden aus der internen Befund-Datenbank des Instituts für Pathologie (Universitätsmedizin Göttingen) ausgewählt. Voraussetzung war eine zuvor in der Routinediagnostik gestellte MDS-Diagnose. Eingeschlossen wurden Patienten, für die im Rahmen der histologischen Materialgewinnung (Beckenkammstanze) auch eine Blutentnahme mit Laboruntersuchung sowie eine genetische Untersuchung (bevorzugt mittels Karyogramm, alternativ mittels FISH) durchgeführt wurde. Üblicherweise erfolgte die Gewinnung aller Proben innerhalb eines diagnostischen Prozesses in der Klinik für Hämatologie und Onkologie. Ausgeschlossen wurden Patienten, bei denen Labor, Histologiebefund oder genetische Analysen nicht vorlagen, beispielsweise, wenn nur einzelne dieser diagnostischen Bausteine von externen Behandlern eingesandt worden waren. Die zu den Patienten gehörenden Präparate wurden aus dem Schnittarchiv gesammelt. Eingeschlossen wurden Präparate aus dem Zeitraum zwischen 2008 und 2014. Patienten mit sequentiellen Biopsien wurden, sofern keine Ausschlusskriterien galten, in das Kollektiv übernommen. Aufgrund der Verblindung (s.u.) wurden sie wie neue Patienten evaluiert. Abbildung 7 zeigt in einer Übersicht den Studienaufbau mit den Ein-/Ausschlusskriterien sowie den sich ergebenden Kollektiven. 


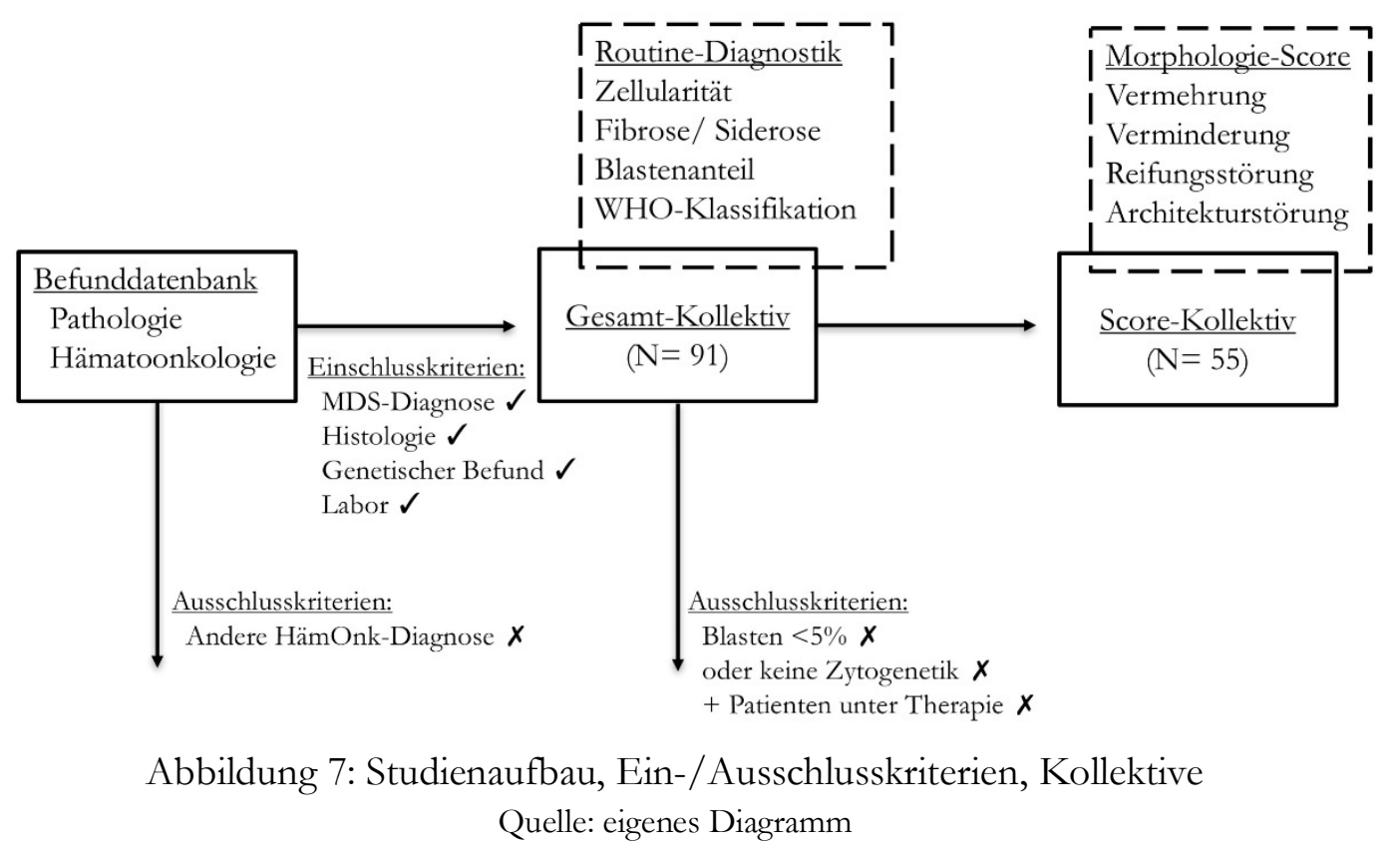

\subsubsection{Demographische Patientendaten}

Die Patientendaten wurden mit Hilfe der in der Klinikdatenbank (iXserv, ixmid Software Technologie GmbH, Köln) hinterlegten Befunde (Pathologie, Labormedizin, Zytogenetik, Arztbriefe) erhoben und in bestimmten Fällen kategorisiert. Jedem Patienten wurde eine Identifikationsnummer (ID) zugewiesen. Diese diente der Anonymisierung von sensiblen Patientendaten (Nachname, Vorname, Geburtsdatum). Die bei Eingang von Gewebeproben in der Pathologie fortlaufend vergebene Präparate-Nummer wurde erfasst. Das Alter des Patienten zum Zeitpunkt der Probenentnahme wurde anhand des Geburtsdatums sowie des Befunddatums errechnet. Das Geschlecht der Patienten wurde registriert.

\subsection{Labordaten}

Folgende, für die Bewertung der histologischen Präparate im Rahmen einer MDS-Abklärung relevanten hämatologischen Laborparameter wurden aus der Befunddatenbank iXserv übertragen: Hämoglobin (in g/dl), Hämatokrit (in \%), Erythrozyten (in 10\% $/ \mu \mathrm{l}$ ), MCV (mean corpuscular volume) (in fl), Thrombozyten (in $10^{3} / \mu \mathrm{l}$ ), Leukozyten (in $10^{3} / \mu \mathrm{l}$ ). Bei Erfassung wurde auf einen engen zeitlichen Zusammenhang zum Entnahmedatum von Histologie und Zytogenetik geachtet.

\subsection{Zytogenetischer Befund}

Die zytogenetischen Daten wurden anhand der im Labor für Zytogenetik der Klinik für Hämatologie und Onkologie (Universitätsmedizin Göttingen) erstellten Befunde erhoben. Hier 
wurden die entsprechenden Untersuchungen im Rahmen der klinischen Routine durchgeführt. Zwei Methoden ließen sich abgrenzen: im Normalfall erfolgte eine Bänderungsanalyse im Karyogramm, unter besonderen Umständen auch die Fluoreszenz-in-situ-Hybridisierung (FISH)-Sondenmarkierung an CD34+-Zellen.

\subsubsection{Karyogramm-Befund und ISCN}

Zur Analyse zytogenetischer Veränderungen wird klassischerweise ein Karyogramm, also die Darstellung einzelner Chromosomenpaare in einer Übersicht, verwendet. Hierfür werden die Knochenmarkszellen erst zur Teilung gebracht und im Metaphasestadium arretiert. In diesem ist die Kondensation des Chromatins am stärksten, sodass nach Fotographie unter dem Mikroskop und Anordnung der Chromosomen in Paaren eine Chromosomen-BänderungsAnalyse (CBA) gut durchgeführt werden kann. Die Zusammenfassung dieser Analyse erfolgt anhand des International System for Human Cytogenetic Nomenclature (ISCN) (Shaffer et al. 2013). Bei der ISCN handelt es sich um ein einheitliches Schema zur Beschreibung von normalen und abnormen Karyogrammen. Die in der ISCN-Formel zuerst genannte Zahl entspricht der absoluten Chromosomenzahl. Nach einem Komma folgen die Geschlechtschromosomen. Liegen zusätzlich strukturelle oder numerische Chromosomenaberrationen vor, werden diese im Anschluss angegeben. Für die Art der Aberration wird eine Abkürzung verwendet (bspw. „del“ für Deletion oder „ins“ für Insertion). Der Ort der Aberration wird ebenfalls durch eine standardisierte Formel angegeben: In der auf die Abkürzung folgende Klammer steht das betroffene Chromosom, in der zweiten Klammer der genaue Ort ( $\mathrm{p} / \mathrm{q}$ für kurzer/langer Arm, die danach folgenden Zahlen die Region, Bande und Subbande). Die bei MDS häufig anzutreffende Mosaikkonstellation wird mit zwei durch ein „/“ getrennte Formeln angegeben. Die Anzahl der untersuchten und betroffenen Metaphasen wird jeweils in eckigen Klammern angegeben.

Die im ISCN enthaltenen genetischen Informationen wurden für die weitere Auswertung kategorisiert und folgende Variablen erfasst: die Anzahl aller untersuchten Metaphasen (MPG), die Anzahl der abnormen Metaphasen (MPA), die Klongröße in \% (KG) und das Vorliegen von komplexen Aberrationen.

\subsubsection{FISH-Sondenpanel an $\mathrm{CD}^{+} 4^{+}$-Zellen}

Wenn eine aktuelle Zytogenetik mittels Karyogramm nicht vorlag, wurde eine aktuelle Verlaufskontrolle mittels FISH an CD34 ${ }^{+}$peripheren Blut- oder Knochenmarkszellen (pB- bzw. KM-Zellen) durchgeführt. Bei ersterem handelt es sich um eine neue Methode in der MDS- 
Diagnostik (Braulke et al. 2010). Aus dem peripheren Blut wurden CD34 ${ }^{+}$-Zellen mittels immunomagnetischer Zellseparation (MACS ${ }^{\circledR}$, Miltenyi Biotex GmbH, Bergisch Gladbach) gewonnen. Anschließend wurden die Zellen mit Hilfe eines FISH-Sondenpanels untersucht (Abbott ${ }^{\circledR}$ Products, Abott GmbH \& Company, KG, Wiesbaden). Bei der FISH wurden mit einem Fluoreszenzfarbstoff verbundene einzelne DNA-Stränge (Sonden) mit den zu untersuchenden Chromosomen vermischt. Binden die Sonden an ihre passenden, komplementären Chromosomenabschnitte, kann dies unter einem Fluoreszenzmikroskop nachgewiesen werden.

\subsubsection{Zytogenetische Risikogruppen des IPSS-R}

Den ermittelten Aberrationen wurde im Rahmen der vorliegenden Dissertation in der Unterkategorie „zytogenetische Risikogruppe“ im IPSS-R eine bestimmte Punktzahl zugewiesen, die neben den Kriterien des Blastenanteils und der Zytopenien in den Gesamt-IPSS-R einging. Nach der aktuellen Revision wurde dies in 5 verschiedene Kategorien mit unterschiedlicher Punktzahl (0 - 4 Punkte) eingeteilt (Greenberg et al. 2012). Die Grundlage des revidierten IPSS mit dem Einschluss neuer Aberrationen bildet das MDS yytogenetic scoring system (Schanz et al. 2012). Tabelle 3 zeigt die zytogenetischen Befund-Konstellationen mit der dazugehörigen Risikoeinstufung (Punktzahl für Berechnung des Gesamt-IPSS-R). Bei Erwähnung des IPSS-(R) ist immer auf die Unterscheidung zwischen dem Gesamt IPSS-(R) und dem jeweiligen zytogenetischen Risikoscore als Unterkategorie des IPSS-(R) zu achten. 
Tabelle 3: Zytogenetische Risikogruppen des IPSS-R

\begin{tabular}{|c|c|c|c|}
\hline Prognostische Subgruppen & Zytogenetis & he Aberrationen & \\
\hline & einfach & doppelt & komplex \\
\hline sehr günstig (0 Pkt.) & $\begin{array}{l}\operatorname{del}(11 q) \\
-Y\end{array}$ & & \\
\hline günstig (1 Pkt.) & $\begin{array}{l}\text { normal } \\
\operatorname{del}(5 \mathrm{q}) \\
\operatorname{del}(12 \mathrm{p}) \\
\operatorname{del}(20 \mathrm{q})\end{array}$ & jede mit del $(5 q)$ & \\
\hline intermediär (2 Pkt.) & 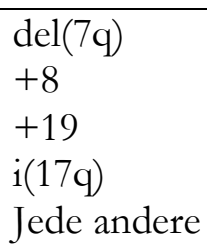 & jede andere & \\
\hline ungünstig (3 Pkt.) & $\begin{array}{l}-7 \\
\text { inv }(3) \\
/ t(3 q) \\
/ \operatorname{del}(3 q)\end{array}$ & jede mit $-7 / \operatorname{del}(7 q)$ & 3 Aberrationen \\
\hline sehr ungünstig (4 Pkt.) & & & $>3$ Aberrationen \\
\hline
\end{tabular}

Modifiziert nach Schanz et al. (2012) und Greenberg et al. (2012)

\subsection{Klinische Angaben}

$\mathrm{Zu}$ jeder eingesendeten Knochenmarksstanze gehörte ein begleitender Text für klinische Angaben und die Fragestellung. Dieser sollte Zeitpunkt sowie Stellenwert der pathologischen Untersuchung im gesamten diagnostischen Prozess einordnen und war Ausgangspunkt für die weitere Diagnostik. Die vielfältigen verschiedenen Anfragen wurden für die statistische Auswertung in relevante Kategorien eingeteilt.

Die Gruppe „V.a. MDS“ (Verdacht auf MDS) umfasste alle Anfragen, bei denen laut Einsendeschein noch kein MDS bekannt war, aber der klinische Verdacht bestand. Ein häufiger Grund für die Bitte um pathologische Mitbeurteilung in dieser Kategorie waren beispielsweise unklare Zytopenien. Von dieser Gruppe abzugrenzen waren Patienten mit bekanntem MDS und einer Verlaufskontrolle. Grund für eine erneute Probenentnahme war hier die Abklärung des Krankheitsverlaufs (Remission, Progress). Da eine Chemotherapie Einfluss auf die Morphologie des Knochenmarks haben kann, wurden verschiedene Gruppen gebildet: „Verlaufskontrolle ohne Therapie“ (für Patienten mit einem watch-and-wait-Ansatz oder rein supportiver Therapie) und „Verlaufskontrolle unter Therapie“ (für Patienten mit aktueller oder stattgehabter Chemotherapie bzw. Stammzelltransplantation). Es sei an dieser Stelle darauf hingewiesen, dass die supportive Therapie, beispielsweise mit Eisenchelatoren 
oder Wachstumsfaktoren, nicht separat erfasst wurde, obwohl diese auch einen Einfluss auf die Knochenmarkshistologie haben kann.

Des Weiteren ließen sich aus dem Einsendeschein oder aus der Krankenakte Hinweise auf die Ätiologie des MDS ziehen. Eine vorherige Radio-Chemotherapie in der Anamnese für eine andere maligne Erkrankung wurde als Therapie-assoziiertes, sekundäres MDS (t-MDS) gewertet.

\subsection{Histologische Präparate}

\subsubsection{Materialgewinnung und Fixierung}

Die histologischen Schnitte der vorliegenden Dissertation wurden im Rahmen der klinischen Routine in der Abteilung für Hämatologie und Onkologie der Universitätsmedizin Göttingen gewonnen und dem Institut für Pathologie zur Mitbeurteilung übersandt. Bei den Gewebeproben handelt es sich um standardmäßig am Beckenkam gewonnene Knochenmarksstanzzylinder (Bain 2001). Hierfür wird der Patient mit angezogenen Beinen in Seitenlage positioniert. Nach Desinfektion sowie Lokalanästhesie von der Haut bis zum Periost wird das Mark auf Höhe der Spina illiaca posterior superior punktiert. Es erfolgt zuerst eine Aspiration von Knochenmark für die zytologische Beurteilung und im Anschluss eine Stanze für die Beurteilung des gesamten Gewebezylinders in der Pathologie. Der $2-3 \mathrm{~mm}$ dicke und 20 $30 \mathrm{~mm}$ lange Stanzylinder wird nun für den Transport und die Zeit bis zur Weiterverarbeitung in einer Fixierungslösung (4\% Formalin) eingelegt. Diese soll eine Zersetzung des Materials verhindern und eventuell vorhandene Krankheitserreger abtöten.

\subsubsection{Blockherstellung, Schneiden und Färben}

Auch die Herstellung der zu beurteilenden Präparate erfolgte in der Routinediagnostik durch Mitarbeiter des Instituts für Pathologie. Um den Knochenmarkszylinder schneiden und färben zu können, ist zunächst eine Entkalkung notwendig (Mulisch und Welsch 2010). Hierfür wird die Probe für 8 Stunden in Ameisensäure eingelegt. Nach Entwässerung des Präparates durch eine aufsteigende Alkoholreihe folgt die Einbettung in Paraffin. Der die Knochenmarksstanze enthaltende Paraffinblock wird mit einem Mikrotom geschnitten. Für den Färbeprozess wird der Schnitt wieder entparaffiniert und Wasser durch eine Alkoholreihe in absteigender Konzentration hinzugefügt. Dann folgt das Färben der Schnitte mit Lösungen, die auf eine bestimmte Weise mit Gewebeanteilen reagieren (siehe Kapitel 2.5.3). Vor einer 
Begutachtung wird dem Präparat durch eine aufsteigende Alkoholreihe wieder Wasser entzogen und das gefärbte Material mit einem Deckglas versiegelt.

\subsubsection{Färbungen bei MDS-Diagnostik}

Die Begutachtung von Knochenstanzzylindern bei MDS erfolgt mit einem bestimmten Panel an Färbungen. Diese unterscheiden sich bezüglich des chemischen Mechanismus und der Anfärbeeigenschaften. Die Durchführung ist in Mulisch und Welsch (2010) detailliert beschrieben.

\subsubsection{Standard- und Spezialfärbungen}

Die Hämatoxylin-Eosin-Färbung (HE) ist eine verbreitete Routine-Standard-Färbung, die eine Übersicht über alle histologischen Präparate ermöglicht. Dies ist auf ihre schnelle und einfache Anwendbarkeit sowie den guten Farbkontrast zurückzuführen. Der HämatoxylinAnteil färbt die anionischen (basophilen) Strukturen, wie Zellkerne und Proteine im Zytoplasma, blauviolett ein, während Eosin als kationischer (eosinophiler) Farbstoff an die sauren Anteile des Zytoplasmas und des Extrazellulärraumes (Kollagen) bindet.

Die Giemsa-Färbung (Gi) ist eine häufig in Blutausstrichen verwendete Färbung und enthält eine Mischung aus Eosin, Methylenblau, Methylenazur und Methylenviolett. Hierdurch kommt ein differenziertes Bild der verschiedenen Blutbestandteile zustande, so zum Beispiel eine bessere Unterscheidung zwischen eosinophilen, basophilen und neutrophilen Granulozyten-Granula.

Die Masson-Goldner-Färbung (Go) gehört zu den Trichromfärbungen, die insbesondere zur Darstellung von Bindegewebe verwendet werden. Die drei eingesetzten Farbstoffe („Trichrom-") haben unterschiedliche Diffusionseigenschaften in den verschiedenen Geweben. So wird ein Farbstoff zwischen zwei Färbevorgängen schneller aus dem Extrazellularraum entfernt als aus dem Zytoplasma. Dadurch kommt es bei der Masson-Goldner-Färbung zu einer abgestuften Färbung mit den Zellkernen in dunkelbraun, dem Zytoplasma in rot und dem Bindegewebe in grün.

Bei der Versilberung handelt es sich um eine Metallimprägnation. Retikuläre Fasern (vor allem Kollagen Typ III) werden dargestellt.

Die Berliner-Blau-Reaktion (Fe) ist die üblicherweise zum Nachweis von Eisen verwendete Färbung. Nur dreiwertiges, ionisiertes Eisen $\left(\mathrm{Fe}^{+}\right)$reagiert hierbei, also kein Eisen, das in organische Verbindungen (bspw. Hämoglobin) eingebaut ist und kein zweiwertiges Eisen. Im Präparat zeigen sich blaue Körnchen als Zeichen der Eisenablagerung. 
Die PAS-Reaktion (periodic acid Schiff reaction) ist eine histochemische Färbung zur Darstellung von Kohlenhydraten. Durch die Periodsäure werden diese zu Aldehyden oxidiert, die wiederum mit einer fuchsinschwefligen Säure (Schiffsches Reagens) unter Freisetzung von Fuchsin reagiert. Dieses ist im Präparat als rot-magenta farbiges Pigment zu sehen.

Der Nachweis von Chloracetatesterase (CAE) gelingt mit der Naphthol-AS-D-ChlorazetatEsterase (NACE)-Reaktion. Neutrophile Granulozyten werden hierdurch violett bis karminrot dargestellt.

\subsubsection{Immunhistochemische Färbungen}

Grundlage für die immunhistochemischen Färbungen ist die Bindung eines aufgebrachten Antikörpers an ein sich im Präparat befindliches Antigen (Epitop). Als nächstes folgt eine optische Markierung dieser Antigen-Antikörper-Verbindung durch einen sekundären Antikörper. In der vorliegenden Arbeit wurde für diesen Prozess das Dako Autostainer Link 48 System (Dako Deutschland GmbH, Hamburg) mit den dazugehörigen Reagenzien verwendet. Zuerst wurde eine hitzeinduzierte Epitopdemaskierung, die die Antigen-Antikörper-Bindung erleichtern sollte, durchgeführt. Eine weitere vorbereitende Behandlung erfolgte durch Inkubation in einer Citratlösung mit einem pH 6 für 20 min, bei CD34, CD61, Glycophorin C und MPO zusätzlich mit einem Mausverstärker („M“, EnVision ${ }^{\mathrm{TM}}$ FLEX+, Mouse (LINKER)) mit Stabilisierungsproteinen für 10 min. Dann wurde der jeweilige primäre Antikörper in Form eines spezifischen Klons (Tabelle 4) aufgetragen, damit dieser an das zu untersuchende Zielantigen band. Die Applikation erfolgte bei manchen Antikörpern unverdünnt (ready to use), bei in konzentrierter Form vorliegendem Reagenz nach entsprechender Verdünnung.

Als sekundärer Antikörper wurde das EnVision ${ }^{\text {TM }}$ System (Abbildung 8) genutzt (Sabattini et al. 1998). Das Grundgerüst bildete ein Dextran-Polymer, an das verschiedene sekundäre Antikörper (von Maus und Kaninchen) angelagert waren (Dako, EnVision ${ }^{\text {TM }}$ FLEX/HRP Detection Reagent, Bestell-Nr. SM802). Diese erhöhte Antikörper-Dichte steigerte die Sensitivität für im Präparat vorhandene Epitope. Außerdem waren an das Dextran-Polymer mehrere HRP-Enzyme (horseradish peroxidase) gebunden. Durch Zugabe des HRP-Substrates DAB (3,3'-Diaminobenzidin Tetrahydrochlorid), wird dieses durch Oxidation braun verfärbt und kann lichtmikroskopisch detektiert werden. Vorteil dieses Systems war zum einen, dass nur zwei Arbeitsschritte notwendig waren (Aufbringen von primärem AK und Polymer), und zum anderen, dass durch die Vielzahl von am Polymer gebundenen Enzymen und Antiköpern eine erhöhte Reaktivität bestand. Die Größe hat jedoch auch mögliche Nachteile beim Ein- und Durchdringen bestimmter Gewebe. 


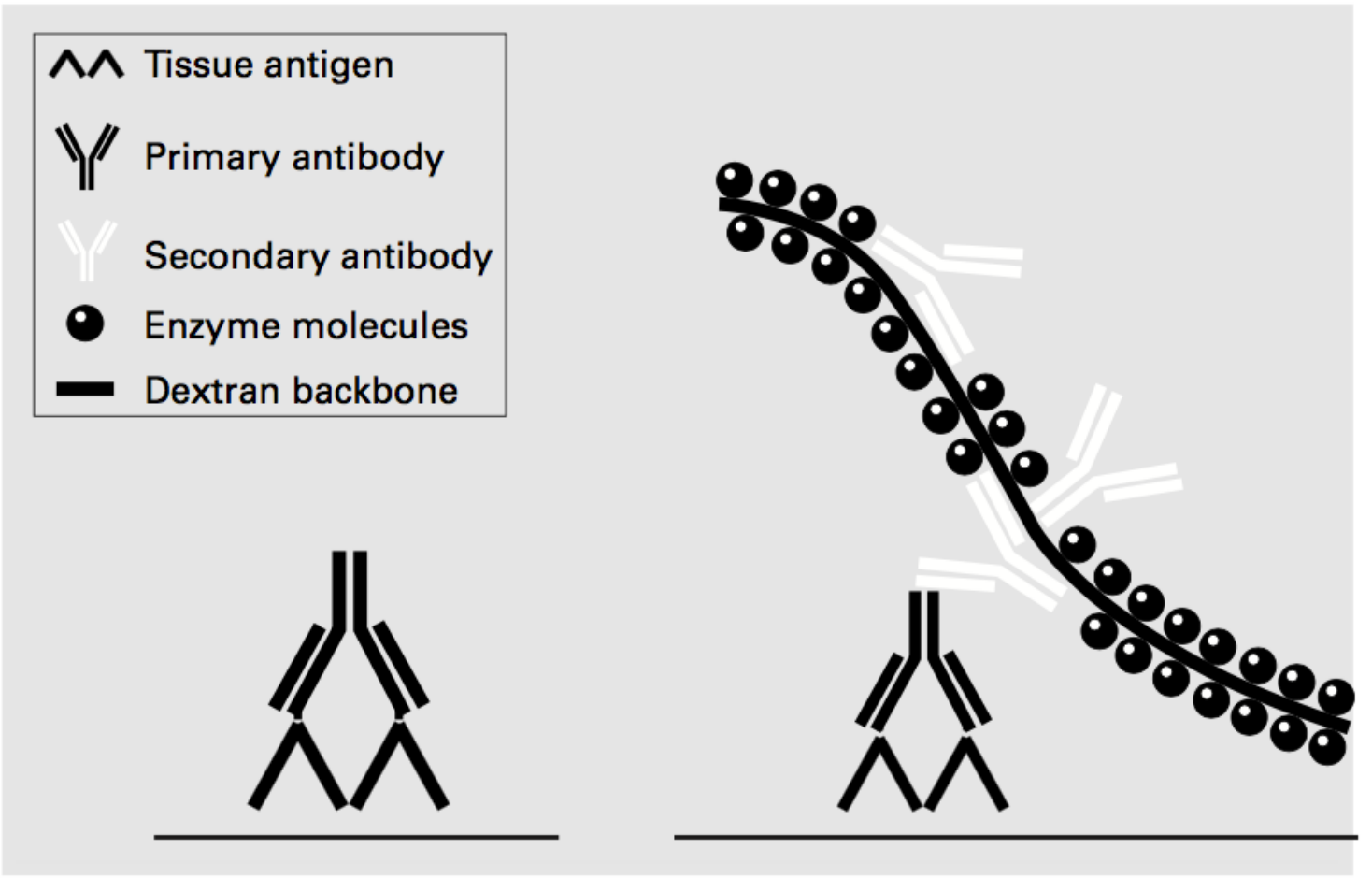

Abbildung 8: Schema der EnVision ${ }^{\text {TM}}$-Antikörper

Übersetzung: Tissue antigen: Gewebsantigen, Primary antibody: primärer Antikörper, Secondary antibody: sekundärer Antikörper, Ensyme molecules: Enzymmoleküle, Dextran backbone: Dextrangrundgerüst; aus Sabattini et al. 1998) mit freundlicher Genehmigung von BMJ Publishing Group Ltd.

Tabelle 4: Immunhistochemische Färbungen bei MDS

\begin{tabular}{|l|l|l|l|l|}
\hline Zielantigen & Klon & $\begin{array}{l}\text { Hersteller } \\
\text { (Bestell-Nr.) }\end{array}$ & Vorbehandlung & Verdünnung \\
\hline CD34 & QBEnd10 & Dako (IR632) & 20 min + M (10 min) & ready to use \\
\hline CD117 & c-kit & Dako (A45020) & 20 min & $1: 500$ \\
\hline CD61 & Y2/51 & Dako (M0753) & 20 min + M (10 min) & $1: 100$ \\
\hline CD3 & & Dako (IR503) & 20 min & ready to use \\
\hline CD20 & L-26 & Dako (IR604) & 20 min + M (10 min) & ready to use \\
\hline Glycophorin C & Ret40f & Dako (M0820) & 20 min + M (10 min) & $1: 100$ \\
\hline MPO & & Dako (IR511) & 20 min + M (10 min) & ready to use \\
\hline
\end{tabular}

Legende: „M“: Mausverstärker 


\subsection{Erste Durchsicht: Routine-Befundung}

Im ersten Untersuchungsdurchgang wurden die Präparate der eingeschlossenen Patienten befundet, wie es in der routinemäßigen Beurteilung von Patienten mit Verdacht auf MDS üblich ist. Hierbei erfolgte eine Verblindung gegenüber den klinischen Angaben, der Zytogenetik sowie der erstmalig gestellten Diagnose. Lediglich Alter, Geschlecht und Laborparameter wurden mitgeteilt. Die Untersuchung und Befundung der Präparate erfolgte nach einem standardisierten Protokoll. Es erfolgte ausschließlich eine histologische Beurteilung der Stanzen und nicht der Ausstriche. Verwendet wurde ein Mikroskop vom Typ Olympus BX53 (Olympus Deutschland GmbH, Hamburg). Kennzeichnend für das histologische Bild bei MDS allgemein ist zum einen das vermehrte Vorliegen einer oder mehrerer Blutzellreihen (bei bekannter peripherer Zytopenie) und zum anderen morphologische Zeichen einer Fehlentwicklung (Dysplasie) der Hämatopoiese. Diese beiden Kriterien wurden bei der Beurteilung der Präparate in verschiedener Weise angewandt.

\subsubsection{Gesamt-Zellularität und Alterskorrektur}

Am Anfang der Beurteilung eines MDS-Präparates stand die Bestimmung der Zellularität, also des Anteils der zellulären Bestandteile am Gesamtmark. Aufgrund der physiologischen Abnahme des blutbildenden Gewebes im Laufe des Lebens war eine altersabhängige Einordnung dieses Wertes notwendig. Die europäischen Konsensus-Richtlinien (Thiele et al. 2005) geben hierzu die in Tabelle 5 dargestellten Empfehlungen. In der vorliegenden Dissertation wurde zur Abschätzung folgende, an die Konsensus-Richtlinien angelehnte Formel verwendet: Das Alter +/- 10 entspricht dem alterskorrigierten Anteil des Fettgewebes in Prozent. Daraus ergibt sich der im Normalfall zu erwartende Anteil der Hämatopoiese (100\% - alterskorrigiertes Fettgewebe). Abbildung 9 verdeutlicht dies an vier circa gleichalten Patienten. Bei einem 70-Jährigen wäre ein Fettanteil von 60 - 80 \% und ein Hämatopoiese-Anteil von $20-40 \%$ zu erwarten. Der Unterschied von erwartetem und tatsächlichem Hämatopoiese-Gehalt wurde anhand einer semiquantitativen Skala erfasst (hypozellulär: -1 , normozellulär: 0 , leicht hyperzellulär: +1 , mäßig hyperzellulär: +2 , stark hyperzellulär: +3$)$.

Tabelle 5: Normale Zellularität nach europäischem Konsensus

\begin{tabular}{cc}
\hline Alter & $\begin{array}{c}\% \text { Hämatopoiese } \\
\text { (normal) }\end{array}$ \\
\hline $20-30$ & $60-70$ \\
$40-60$ & $40-50$ \\
$\geq 70$ & $30-40$ \\
\hline \multicolumn{2}{l}{ Modifiziert nach: Thiele et al. (2005) }
\end{tabular}



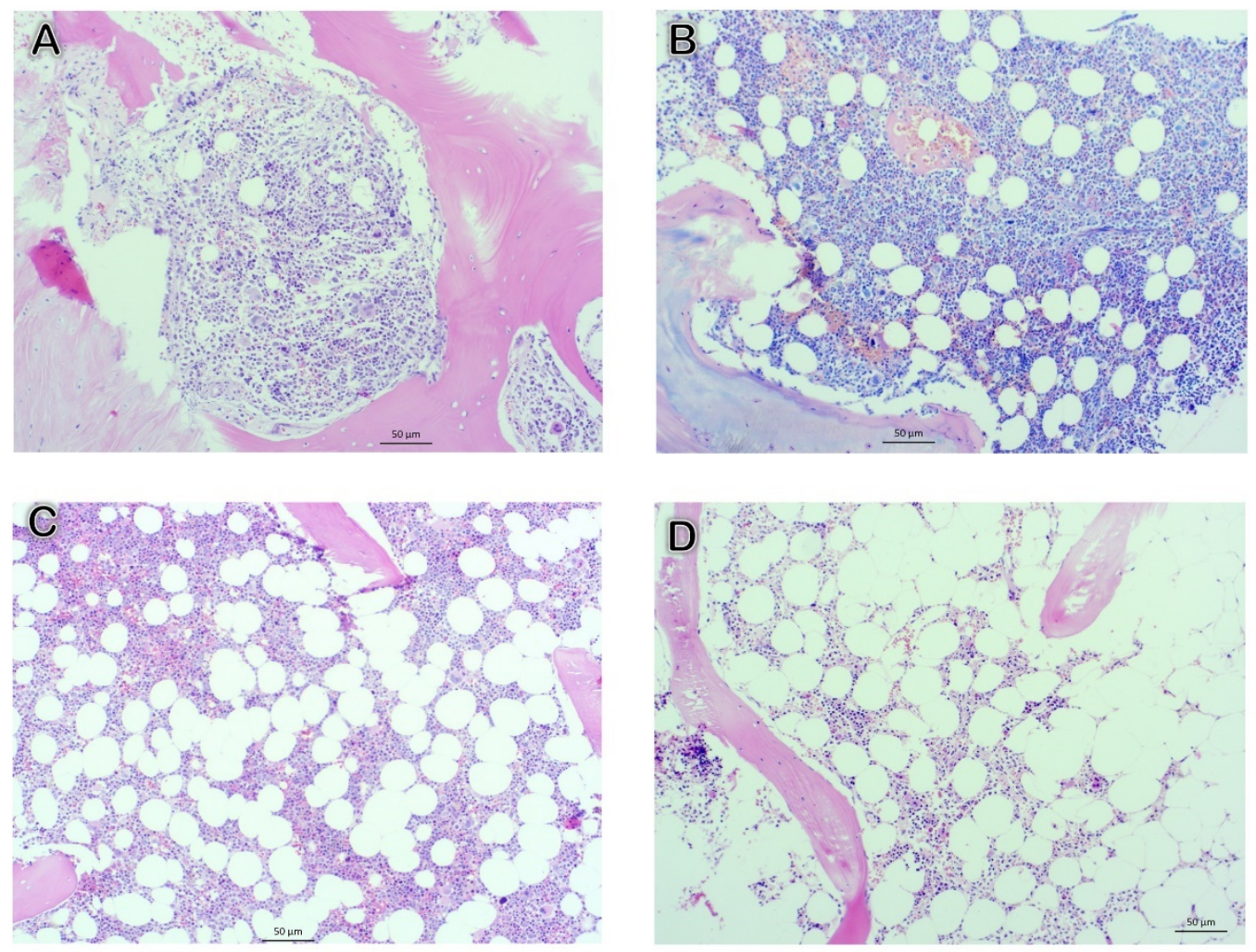

Abbildung 9: Altersabhängige Zellularität

Unterschiede im Hämatopoieseanteil bei vier Patienten derselben Altersgruppe (68-74 Jahre); A: stark hyperplastisches Knochenmark (Grad 3) [Patient ID-37, Hämatoxylin-Eosin-Färbung, 10-fache Vergrößerung], B: mäßig hyperplastisches Knochenmark (Grad 2) [Patient ID-24, Giemsa-Färbung, 10-fache Vergrößerung], C: leicht hyperplastisches Knochenmark (Grad 1) [Patient ID-81, Hämatoxylin-Eosin-Färbung, 10-fache VergröBerung], D: hypoplastisches Knochenmark (Patient ID-51, Hämatoxylin-Eosin-Färbung, 10-fache Vergrößerung); Quelle: Quelle: eigene Präparate

\subsubsection{Markraumsiderose}

Die Beurteilung des Eisengehaltes erfolgte anhand der Berliner-Blau-Reaktion (siehe Kapitel 2.5.3.1). Die pathologische Eisenspeicherung im Knochenmark, die Markraumsiderose, wurde auf einer Skala semiquantitativ erfasst. Die Eisenablagerung wurde in folgende Stufen eingeteilt: 0 = keine, 1 = leicht, 2 = mäßig, $3=$ stark. Beispiele für die verschiedenen Schweregrade sind in Abbildung 10 wiedergegeben. Bei sehr starker Eisenüberladung sind bereits in der HE-Färbung rotbraune Ablagerungen zu erkennen. 

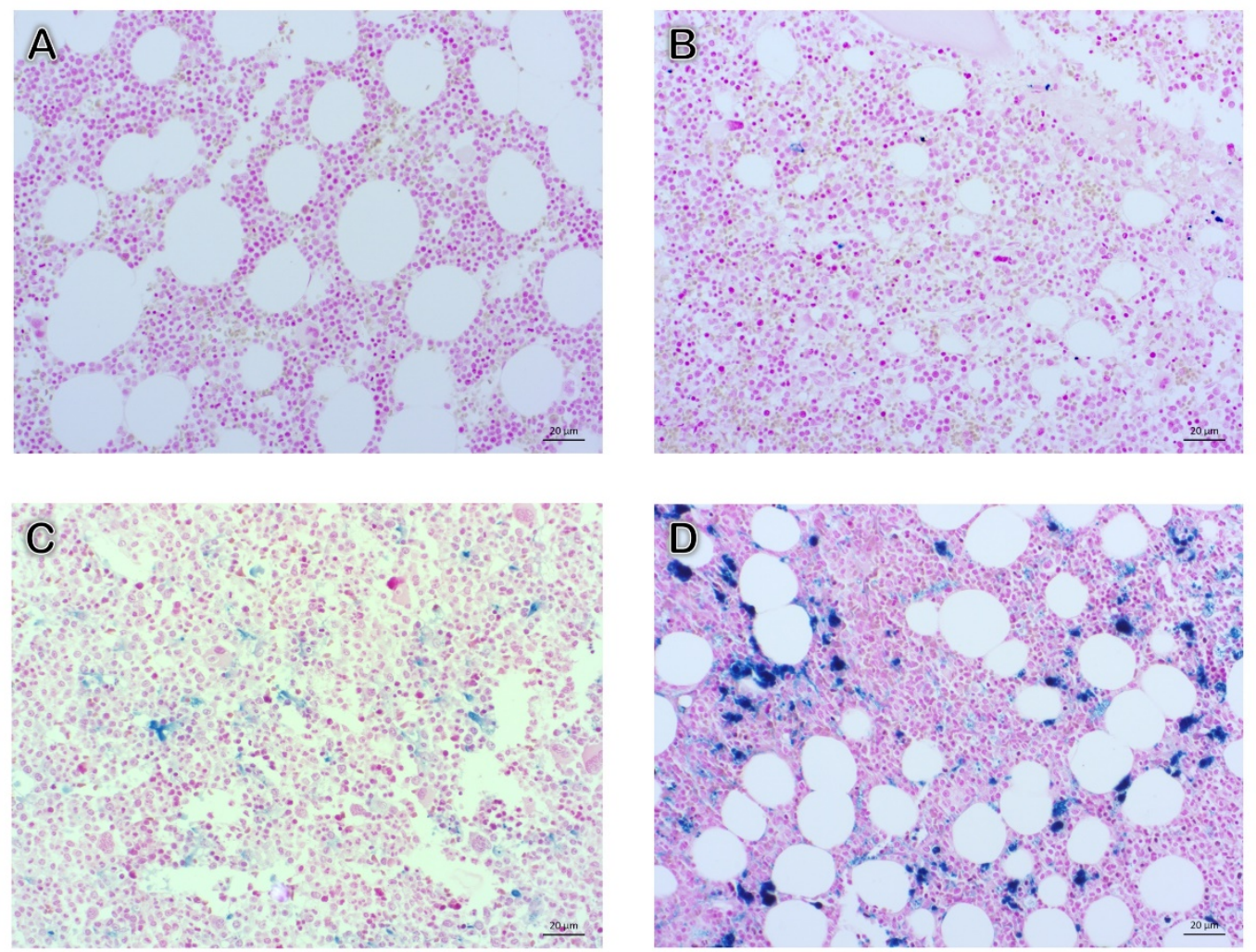

Abbildung 10: Markraumsiderose

A: keine Einlagerung (Grad 0) [Patienten ID-84, Berliner-Blau-Färbung, 20-fache Vergrößerung], B: leichte Eiseneinlagerung (Grad 1) [Patienten ID-10, Berliner-Blau-Färbung, 20-fache Vergrößerung], C: mäßige Eisenlagerung (Grad 2) [Patienten ID-6, Berliner-Blau-Färbung, 20-fache Vergrößerung], D: sehr starke Eiseneinlagerung (Grad 3) Patienten ID-84, Berliner-Blau-Färbung, 20-fache Vergrößerung]; Quelle: eigene Präparate

\subsubsection{Markraumfibrose}

Analog zum Eisen erfolgte eine Quantifizierung der Markraumfibrose (MF), also des vermehrten Vorhandenseins von extrazellulärer Matrix in Form von Retikulin. Am besten geeignet ist eine Versilberung. Die Gradeinteilung der Markraumfibrose erfolgte anhand der Europäischen Konsensus-Leitlinie (Thiele et al. 2005). Diese gibt vor, dass zuerst eine interne Kontrolle der Färbung anhand der Anfärbbarkeit von Gefäßwandungen durchgeführt und dann die Gradeinteilung vorgenommen wird (Tabelle 6). Die unterschiedlichen Ausprägungsgrade sind in Abbildung 11 dargestellt. Ein Patient mit MF-3 befand sich nicht im Kollektiv. 
Tabelle 6: Einteilung der Markraumfibrose nach europäischem Konsensus

\begin{tabular}{cl}
\hline Gradeinteilung & \multicolumn{1}{c}{ Beschreibung } \\
\hline MF-0 & $\begin{array}{l}\text { verteiltes, bandförmiges Retikulin ohne Überschneidungen } \\
\text { (Kreuzungen) wie im normalen Knochenmark }\end{array}$ \\
\hline MF-1 & $\begin{array}{l}\text { lockeres Netzwerk von Retikulin mit vielen Überschnei- } \\
\text { dungen, vor allem perivaskulär }\end{array}$ \\
\hline MF-2 & $\begin{array}{l}\text { diffuse und dichte Vermehrung von Retikulin mit häufigen } \\
\text { Überschneidungen, zum Teil fokale Kollagen-Bündel oder } \\
\\
\text { Osteosklerose }\end{array}$ \\
\hline MF-3 & $\begin{array}{l}\text { diffuse und dichte Vermehrung von Retikulin mit häufigen } \\
\text { Überschneidungen, häufige, grobe Kollagen-Bündel, signi- } \\
\text { fikante Osteosklerose }\end{array}$ \\
\hline
\end{tabular}

Modifiziert nach Thiele et al. (2005)
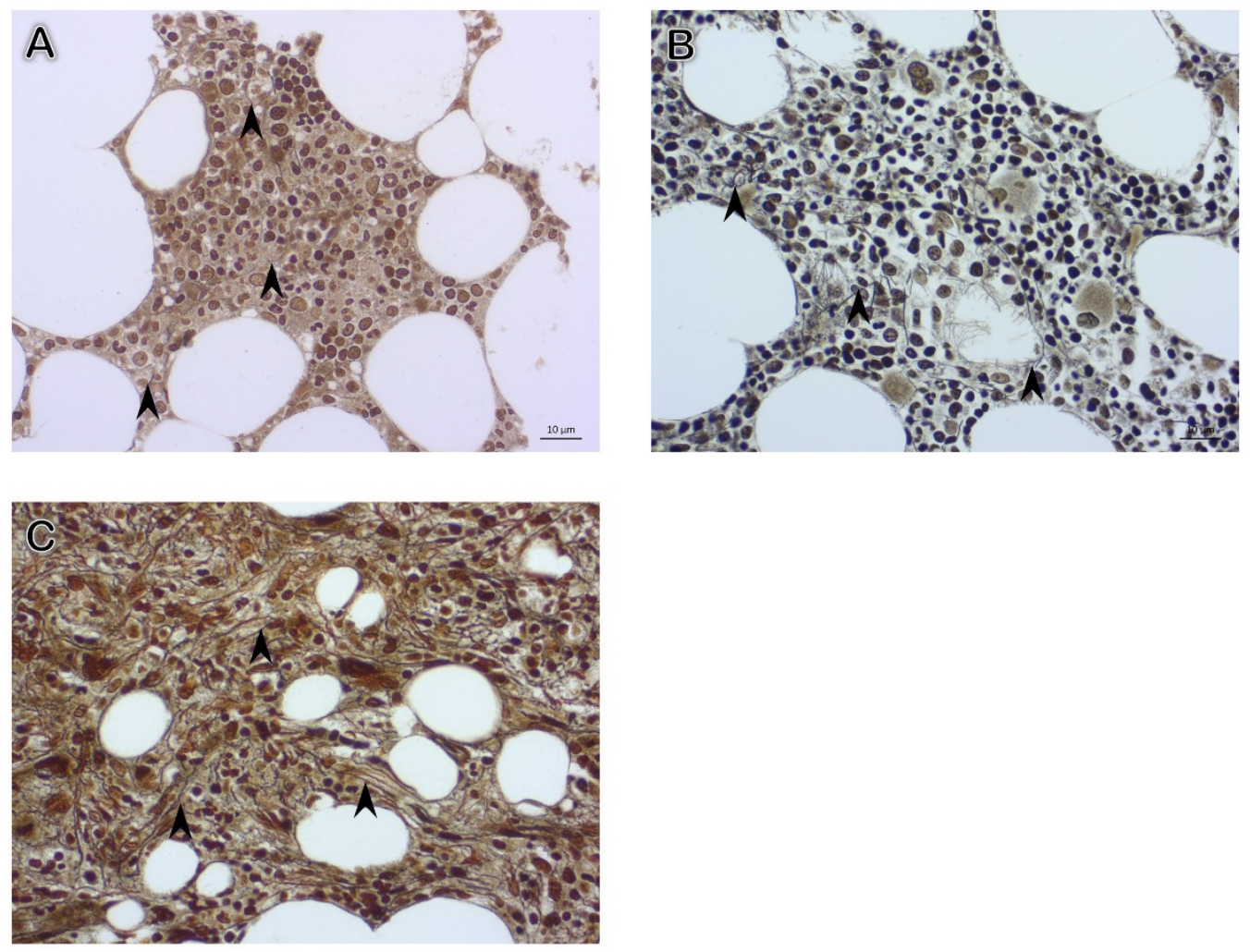

Abbildung 11: Markraumfibrose

A: zartes retikuläres Faserwerk (૪) (Kontrollpatient, Versilberung, 40-fache Vergrößerung), B: leichte Faservermehrung (๖) (Grad 1) (Patient ID-16, Versilberung, 40-fache Vergrößerung), C: mäßige Markraumfibrose (๖) (Grad 2) (Patient ID-40, Versilberung, 40-fache Vergrößerung); Quelle: eigene Präparate 


\subsubsection{Lymphozytenansammlungen}

Das Vorhandensein von Lymphozytenansammlungen wurde erfasst. In Abbildung 12 ist eine solche Population abgebildet. Die CD3-Immunhistochemie konnte für die Darstellung von T-Zellen und die CD20-Immunistochemie für B-Zellen genutzt werden.
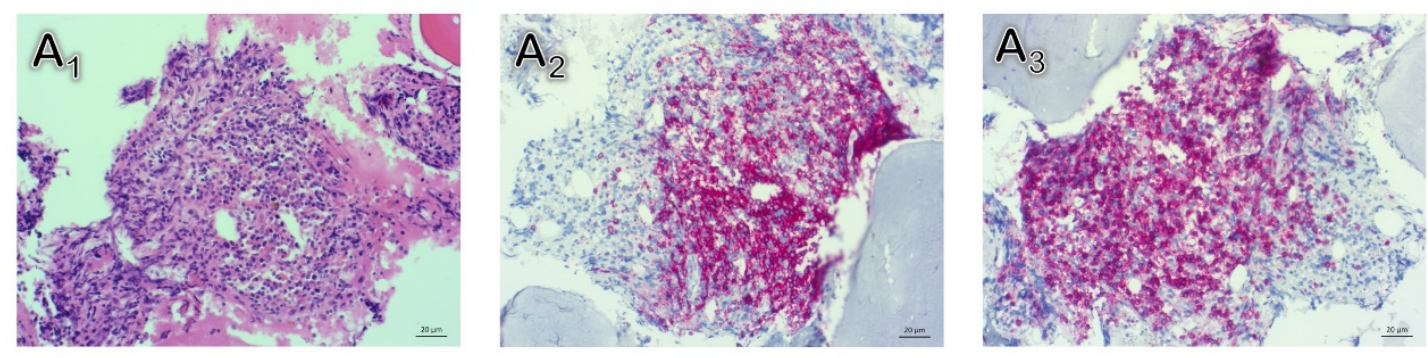

Abbildung 12: Gruppierte Lymphozytenansammlung

A: Lymphozyten-Ansammlung [Patient ID-10, 20-fache Vergrößerung, A1: Hämatoxylin-Eosin-Färbung, A2: CD20-Immunhistochemie, A3: CD3-Immunhistochemie]; Quelle: eigene Präparate

\subsubsection{Qualitative morphologische Beurteilung der Hämatopoiese}

Zur Diagnostik eines histologischen Schnittes bei MDS gehört auch der Versuch, morphologische Veränderungen innerhalb der verschiedenen Zellreihen sowie der gesamten Knochenmarksarchitektur (Dysplasie-Zeichen) festzustellen, wobei dies streng genommen der zytologischen Beurteilung des Knochenmarksausstriches vorbehalten ist. In der pathologischen Routinediagnostik werden diese Veränderungen qualitativ beschrieben und im Freitext der Befundmitteilung erfasst. In dieser Studie wurden im ersten Durchgang Veränderungen in den Kategorien „Erythropoiese“, „Granulopoiese“, „Megakaryopoiese“ sowie „Architektur“ ebenfalls als Freitext festgehalten. Beschrieben wurde beispielsweise die Vermehrung oder Verminderung einer bestimmten Zellreihe, Veränderungen der Morphologie einzelner Zellen (Atypien, Mitosefiguren, Kerngrößenunterschiede, Größenunterschiede zwischen den Zellen, das Auftreten früher, blastärer Entwicklungsstufen) sowie Störungen in der normalen Verteilung der Zellreihen im Knochenmark (Clusterbildung, Auflösung von Erythrozytennestern, Vorhandensein von Granulozyten im Zentrum des Markraums). Diese Beurteilung bildete unter anderem die Grundlage für die Entwicklung der semi-quantitativen Kategorien, mit denen die Knochenmarksmorphologie in der zweiten Durchsicht erfasst wurde. Eine detaillierte Darstellung dieser morphologischen Veränderungen anhand von Bildern erfolgt mit der Besprechung dort.

\subsubsection{Immunhistochemische Blastenbestimmung}

In der vorliegenden Studie wurde der Anteil der Blasten im Vergleich zu allen Zellen in \% bestimmt. Verwendet wurden die Immunfärbungen für $\mathrm{CD} 34^{+}-$und $\mathrm{CD} 117^{+}$-Blasten. 
Abbildung 13 zeigt verschiedene Ausprägungsgrade einer Blastenvermehrung. Der Anteil ist sowohl für die WHO-Klassifikation (5 - $9 \%$ Blasten entspricht MDS-EB-1, $10-20 \%$ entspricht MDS-EB-2) als auch für den IPSS-(R) relevant.
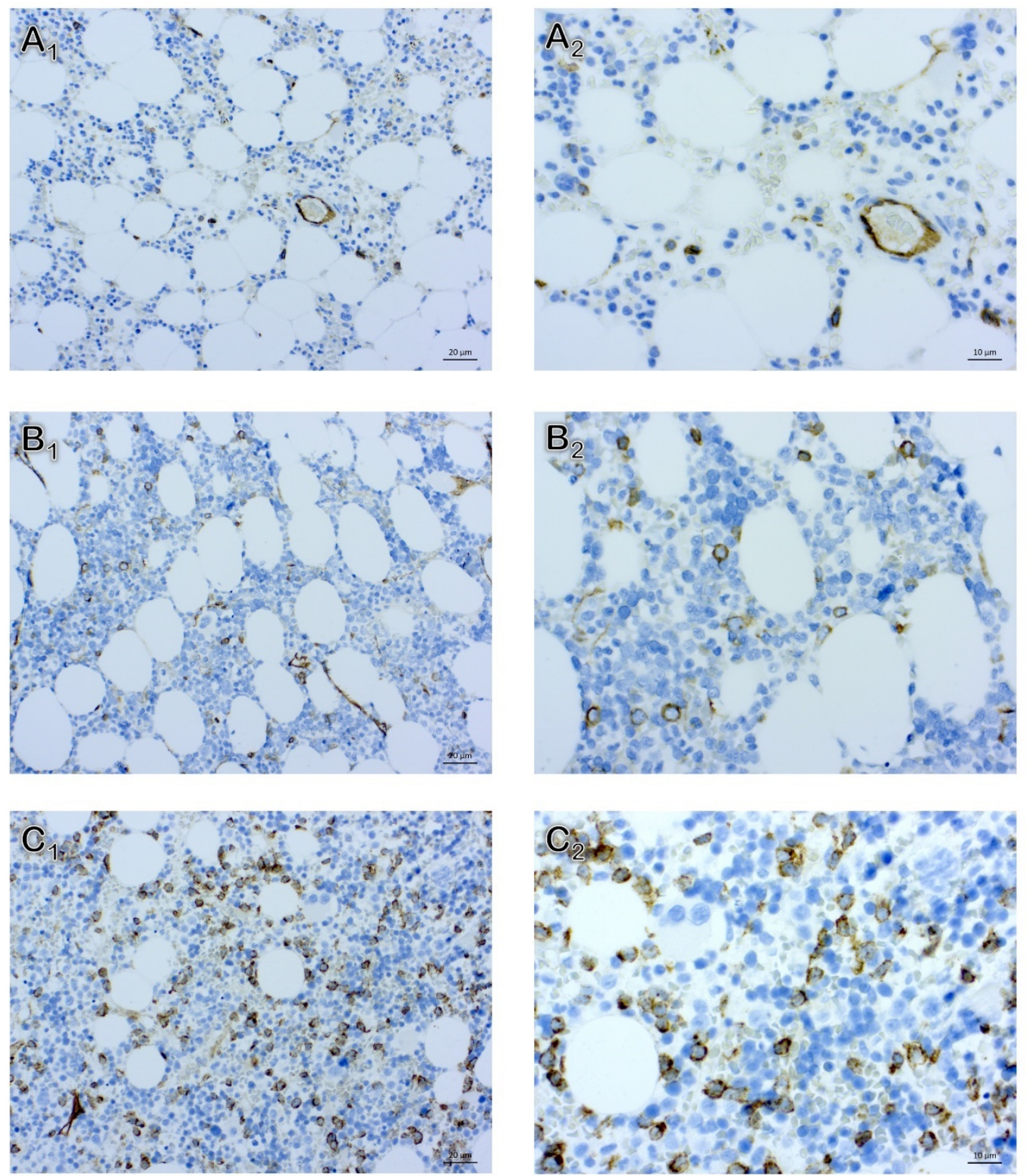

Abbildung 13: Blastenanteil

A: Knochenmark mit unter $5 \%$ CD-34+-Zellen [Patient ID-4, CD-34-Immunhistochemie, $\mathrm{A}_{1}$ : Übersichtsansicht, 20-fache Vergrößerung und $A_{2}$ : Detailansicht, 40-fache Vergrößerung], B: Knochenmark mit 5 - $9 \%$ CD-34+-Zellen (MDS-EB-1) [Patient ID-90, CD-34-Immunhistochemie, B1: Übersichtsansicht, 20-fache Vergrößerung und $\mathrm{B}_{2}$ : Detailansicht, 40-fache Vergrößerung], C: $10-20 \%$ CD-34+-Zellen (MDS-EB-2) [Patient ID-4, CD-34-Immunhistochemie, $C_{1}$ : Übersichtsansicht, 20-fache Vergrößerung und $C_{2}$ : Detailansicht, 40-fache Vergrößerung]; Quelle: eigene Präparate 


\subsubsection{Diagnosestellung nach WHO-Klassifikation}

In Zusammenschau aller Befunde wurde die histomorphologische Studiendiagnose laut WHO-Klassifikation gestellt. Eine Einteilung in die Kategorien MDS-SLD, MDS-MLD, MDS-EB-1 und MDS-EB-2 erfolgte. Eine Einteilung in alle zehn Sub-Kategorien der WHOKlassifikation ist allein basierend auf dem histologischen Präparat nicht möglich. Deshalb orientieren sich die unten genannten Kategorien zwar an der WHO-Klassifikation, sind allerdings weniger detailliert. Die vollständigen, zusammengefassten Diagnosekriterien sind in Tabelle 7 dargestellt.

Die pathologische MDS-SLD-Kategorie (MDS with single lineage dysplasia) beschreibt Patienten mit morphologischen Veränderungen in mehr als $10 \%$ der Zellen einer Zellreihe (Erythropoiese, Granulopoiese, Megakaryopoiese) und Blasten unter $5 \%$, während bei MDS-MLDKategorie (MDS with multilineage dysplasia) mehrere Zellreihen betroffen sind.

Eine weitere Kategorie ist das MDS with ring sideroblasts (MDS-RS), ehemals bezeichnet als RARS (Refraktäre Anämie mit Ring-Sideroblasten), in der > $15 \%$ der Zellen Eisengranula aufweisen. Gewebe dieser MDS-Kategorie wird am besten zytologisch im Knochenmarksausstrich begutachtet, weil die Berliner-Blau-Färbung intrazelluläres Eisen nicht zuverlässig anfärbt (Horny et al. 2007). Deswegen wurde diese Kategorie in der vorliegenden Studie nicht vergeben. Es ist ebenfalls möglich ein MDS-RS zu diagnostizieren, wenn die Ringsideroblasten unter $15 \%$ aber über $5 \%$ liegen, solange zusätzlich eine SF3B1-Mutation vorliegt. In die Kategorien der MDS with excess of blasts (MDS-EB) wird eingeteilt bei unioder multilinärer Dysplasie mit 5 - 9\% Blasten (für MDS-EB-1) oder 10 - $20 \%$ Blasten (für MDS-EB-2).

Für die WHO-Kategorien MDS with isolated del( $5 q$ ) sowie MDS, unclassifiable (MDS-U) müssen zytogenetische Untersuchungen vorliegen, gegenüber denen jedoch bei der Durchsicht verblindet wurde. Beim 5q-Syndrom liegen typische morphologische Veränderungen der Megakaryopoiese vor zusammen mit der entsprechenden isolierten Zytogenetik. In die Gruppe MDS-Unklassifizierbar werden verschiedene Konstellationen eingeordnet (siehe Tabelle 7) (Arber et al. 2016). 
Tabelle 7: Befunde im peripheren Blut und Knochenmark, WHO-Klassifikation 2016

\begin{tabular}{lll}
\hline Entität & Blut-Untersuchung & Knochenmarks-Untersuchung \\
\hline MDS mit unilineärer Dysplasie & Unizytopenie oder Bizytopenie & unilineäre Dysplasie \\
(MDS-SLD) & $<1 \%$ Blasten & $<5 \%$ Blasten \\
& & $<15 \%$ Ringsideroblasten \\
\hline MDS mit multilineärer Dysplasie & Zytopenie(n) & multilineäre Dysplasie \\
(MDS-MLD) & $<1 \%$ Blasten & $<5 \%$ Blasten \\
& & $<15 \%$ Ringsideroblasten \\
\hline
\end{tabular}

MDS mit Ringsideroblasten

(MDS-RS)

\begin{tabular}{|c|c|c|}
\hline $\begin{array}{l}\text { MDS-RS mit unilineärer Dysplasie } \\
\text { (MDS-RS-SLD) }\end{array}$ & $\begin{array}{l}\text { Unizytopenie oder Bizytopenie } \\
<1 \% \text { Blasten }\end{array}$ & $\begin{array}{l}\text { unilineäre Dysplasie } \\
<5 \% \text { Blasten } \\
\geq 15 \% \text { Ringsideroblasten }\end{array}$ \\
\hline $\begin{array}{l}\text { MDS-RS mit multilineärer Dyspla- } \\
\text { sie (MDS-RS-MLD) }\end{array}$ & $\begin{array}{l}\text { Zytopenie }(\mathrm{n}) \\
<1 \% \text { Blasten }\end{array}$ & $\begin{array}{l}\text { multilineäre Dysplasie } \\
<5 \% \text { Blasten } \\
\geq 15 \% \text { Ringsideroblasten }\end{array}$ \\
\hline MDS mit del(5q) & $\begin{array}{l}\text { Unizytopenie oder Bizytopenie } \\
<1 \% \text { Blasten }\end{array}$ & $\begin{array}{l}\text { uni- oder multilineäre Dysplasie } \\
<5 \% \text { Blasten } \\
\operatorname{del}(5 \mathrm{q}) \text { isoliert oder mit einer Zusatz- } \\
\text { anomalie außer }-7 \text { oder del }(7 \mathrm{q})\end{array}$ \\
\hline
\end{tabular}

MDS mit Blastenexzess

(MDS-EB)

\begin{tabular}{|c|c|c|}
\hline MDS-EB-1 & $\begin{array}{l}\text { Zytopenie(n) } \\
2-4 \% \text { Blasten } \\
\text { keine Auer-Stäbchen } \\
\text { Zytopenie(n) } \\
<5-19 \% \text { Blasten } \\
\text { Auer-Stäbchen }\end{array}$ & $\begin{array}{l}\text { keine, uni-/multilineäre Dysplasie } \\
<5-9 \% \text { Blasten } \\
\text { keine Auer-Stäbchen } \\
\text { keine, uni-/multilineäre Dysplasie } \\
<10-19 \% \text { Blasten } \\
\text { Auer-Stäbchen }\end{array}$ \\
\hline \multicolumn{3}{|l|}{ MDS-Unklassifizierbar (MDS-U) } \\
\hline $\begin{array}{l}\text { mit } 1 \% \text { Blasten im Blut } \\
\text { mit unilineärer Dysplasie und } \\
\text { Panzytopenie } \\
\text { basierend auf definierender Zyto- } \\
\text { genetik }\end{array}$ & $\begin{array}{l}\text { Zytopenie(n) } \\
1 \% \text { Blasten } \\
\text { Panzytopenie } \\
<1 \% \text { Blasten } \\
\text { Zytopenie(n) } \\
<1 \% \text { Blasten }\end{array}$ & $\begin{array}{l}\text { uni- oder multilineäre Dysplasie } \\
<5 \% \text { Blasten } \\
\text { unilineäre Dysplasie } \\
<5 \% \text { Blasten } \\
\text { keine Dysplasie } \\
\text { MDS-definierende Zytogenetik } \\
<5 \% \text { Blasten }\end{array}$ \\
\hline $\begin{array}{l}\text { Refraktäre Zytopenie des Kindesal- } \\
\text { ters }\end{array}$ & $\begin{array}{l}\text { Zytopenie(n) } \\
<2 \% \text { Blasten }\end{array}$ & $\begin{array}{l}\text { uni- oder multilineäre Dysplasie } \\
<5 \% \text { Blasten }\end{array}$ \\
\hline
\end{tabular}

Dysplasie liegt vor, wenn $>10 \%$ der Zellen einer Reihe betroffen sind, ein Subtyp mit Ringsideroblasten kann auch vorliegen, wenn $<15 \%$ aber $>5 \%$ der Zellen Eisengranula aufweisen und zusätzliche eine SF3B1Mutation vorliegt. Modifiziert nach Arber et al. (2016)

\subsection{Zweite Durchsicht: Anwendung des Morphologie-Scores}

Um die Aussagekraft der morphologischen Ergebnisse vergleichen zu können, wurden verschiedene Parameter in einer zweiten Durchsicht semi-quantitativ erhoben. Erneut wurde der Befund gegenüber der Diagnose, Zytogenetik und den klinischen Angaben verblindet. Berichtet wurde lediglich Alter, Geschlecht, Laborparameter sowie die im ersten Durchgang ermittelten Kategorien für Knochenmarkssiderose, Fibrose, alterskorrigierte Zellularität sowie Lymphozyten. Der Blastengehalt wurde nicht mitgeteilt und die Beurteilung der CD34- 
Expression von Megakaryozyten ans Ende der Bewertung gestellt, um eine von diesem wichtigen Prognosefaktor unabhängige Beurteilung der morphologischen Kriterien zu gewährleisten. Dieser „Morphologie-Score MDS“ wurde an einem Sub-Kollektiv von mit hoher Sicherheit an MDS erkrankten Patienten durchgeführt. Als diagnostisch sicher in diesem Sinne galt für diese Studie die signifikante Erhöhung von Blasten bei der ersten Durchsicht (> $5 \%$ ) oder der zytogenetische Nachweis einer Aberration. Wegen des möglichen Einflusses der Chemotherapie auf die Morphologie des Knochenmarks wurden die Patienten der Gruppe „Verlaufskontrolle unter Therapie“ von dieser zweiten Durchsicht ausgeschlossen.

\subsubsection{Aufbau des Morphologie-Scores}

Die zu beurteilenden Kriterien ergaben sich aus der klinischen Erfahrung und den im ersten Durchgang registrierten Beobachtungen zu morphologischen Veränderungen bei MDS. Sowohl quantitative Veränderungen in der Zellzahl als auch qualitative Veränderungen wurden erfasst. Letztere wurden noch einmal eingeteilt in Reifungsstörungen, die sich auf Veränderungen in der Morphologie der einzelnen Zelle beziehen, und Architekturstörungen, die auf einer abnormen Verteilung der Zellen im Knochenmark basieren. Die semi-quantitative Einteilung erfolgte bei den Reifungs- und Architekturstörungen jeweils in die Kategorien 0 (normal), 1 (leicht atypisch), 2 (mäßig atypisch) oder 3 (schwer atypisch). Eine höhere Kategorie zeigt also jeweils einen höheren Dysplasie- oder Atypiegrad an.

\begin{tabular}{|c|c|c|c|c|c|c|c|c|c|c|c|c|c|}
\hline & $+/-$ & \multicolumn{4}{|c|}{ Quantität } & \multicolumn{4}{|c|}{ Reifungsstörung } & \multicolumn{4}{|c|}{ Architekturstörung } \\
\hline & & & & & & & & & & & & & \\
\hline Granulopoese & & 0 & 1 & $x$ & 3 & 0 & 1 & 2 & 3 & 0 & 1 & 2 & 3 \\
\hline Linksverschiebung & & & & & & 0 & 1 & $x$ & 3 & & & & \\
\hline Größenvariabilität & & & & & & 0 & $X$ & 2 & 3 & & & & \\
\hline ALIPS & & & & & & & & & & 0 & 1 & 8 & 3 \\
\hline & & & & & & & & & & & & & \\
\hline & & & & & & & & & & & & & \\
\hline Erythropoese & & 0 & X & 2 & 3 & 0 & 1 & 2 & 3 & 0 & 1 & 2 & 3 \\
\hline Makrozytose & & & & & & 0 & 1 & $\frac{x}{3}$ & 3 & & & & \\
\hline Kerngrößen+/- & & & & & & 0 & 1 & K & 3 & & & & \\
\hline Unscharfe Nester & & & & & & & & & & 0 & 1 & $\star \forall$ & 3 \\
\hline & & & & & & & & & & & & ' & \\
\hline Megakaryozyten & & 0 & 1 & 这 & 3 & 0 & 1 & 2 & 3 & 0 & 1 & 2 & 3 \\
\hline Hypolobuliert & & & & & & 0 & 1 & X & 3 & & & & \\
\hline Mikrozytär & & & & & & 0 & 1 & स & 3 & & & & \\
\hline Cluster-Bildung & & & & & & & & & & 0 & 1 & $x$ & 3 \\
\hline CD34+ & & & & & & 0 & $\mathbb{X}$ & 2 & 3 & & & & \\
\hline $\mathrm{Fe}$ & & 0 & 1 & 2 & $\not 8$ & & & & & & & & \\
\hline Faser & & $x$ & 1 & 2 & 3 & & & & & & & & \\
\hline Zellularität & & 0 & 1 & 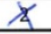 & 3 & & & & & & & & \\
\hline Lymphos & & d & 1 & & & & & & & & & & \\
\hline
\end{tabular}

Abbildung 14: Morphologie-Score MDS

Beispiel eines Morphologie-Scores (Patient mit der Patienten-ID 54); Quelle: eigene Abbildung 


\subsubsection{Quantitative Veränderungen einzelner Zellreihen}

Jede Zellreihe - Granulopoiese, Erythropoiese und Megakaryopoiese - wurde nach Quantität eingeteilt in die Kategorien -3 (stark vermindert), -2 (mäßig vermindert), -1 (leicht vermindert), 0 (normal), +1 (leicht vermehrt), +2 (mäßig vermehrt), +3 (stark vermehrt). Dies ist als ein relativer Wert zu verstehen, zum einen im Vergleich zur Gesamt-Hämatopoiese als auch zu den anderen Zellreihen. In Abbildung 15 ist diese Einordnung an einem Beispiel aufgezeigt. Die Darstellung einer Vermehrung der Megakaryopoiese ist in Abbildung 17B wiedergegeben.
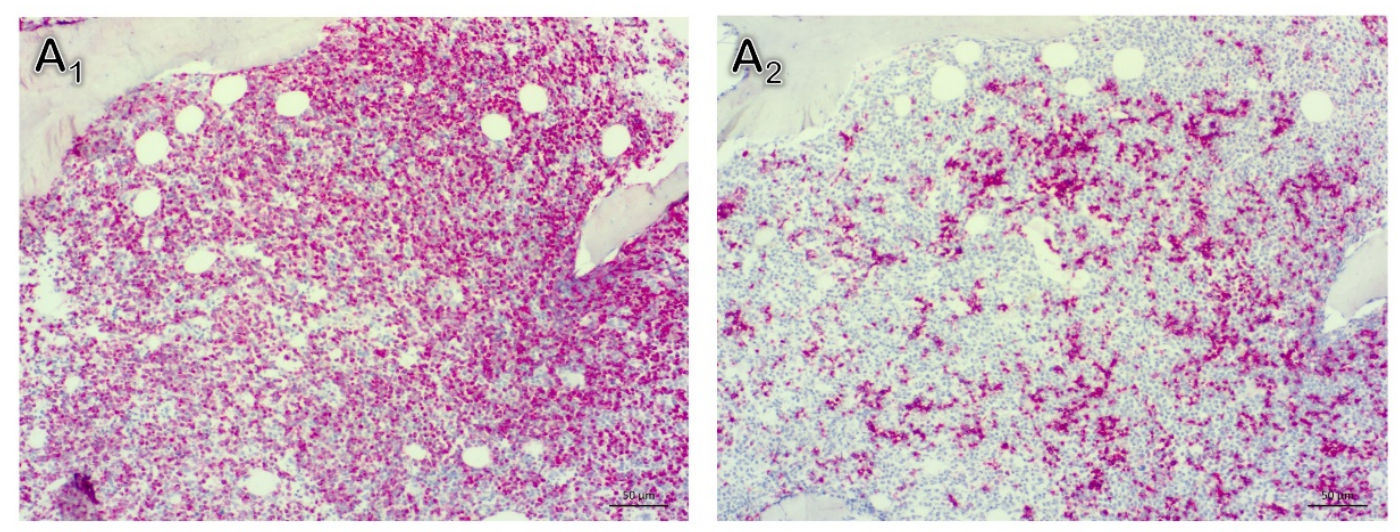

Abbildung 15: Quantitative Veränderungen der Zellreihen

A: quantitative Veränderungen: insgesamt gesteigerte Hämatopoiese Grad 3, starke Steigerung der Granulopoiese $\left(+3 ; A_{1}\right)$ sowie mäßige Steigerung der Erythropoiese $\left(+2 ; A_{2}\right)$, damit ist auch das Verhältnis von Granulopoiese zu Erythropoiese verschoben [Patient ID-44, 10-fache Vergrößerung, A $A_{1}$ : MPOImmunhistochemie; $\mathrm{A}_{2}$ : Glycophorin-C-Immunhistochemie]; Quelle: Quelle: eigene Präparate

\subsubsection{Reifungs- und Architekturstörung der Erythropoiese}

Die Erythrozyten reifen normalerweise in den als Erythronen bezeichneten Nestern mittig im Markraum aus. Im Rahmen von MDS vorkommende Reifungsstörungen wurden eingeteilt nach dem Grad der Makrozytose (vermehrtes Vorkommen uniformer, übergroßer Zellen) und nach Kerngrößenvariabilität (Schwankungen in der Größe der Nuklei) (Abbildung 16).

Die Auflösung der Erythronen, Kennzeichen einer Architekturstörung der erythrozytären Reihe, wurde beurteilt. Hier entstehen meist strangartige Ausläufer oder vereinzelt liegende erythrozytäre Vorstufen. Diese können von der Markraummitte aus in die Nähe der Trabekel verlagert sein. 

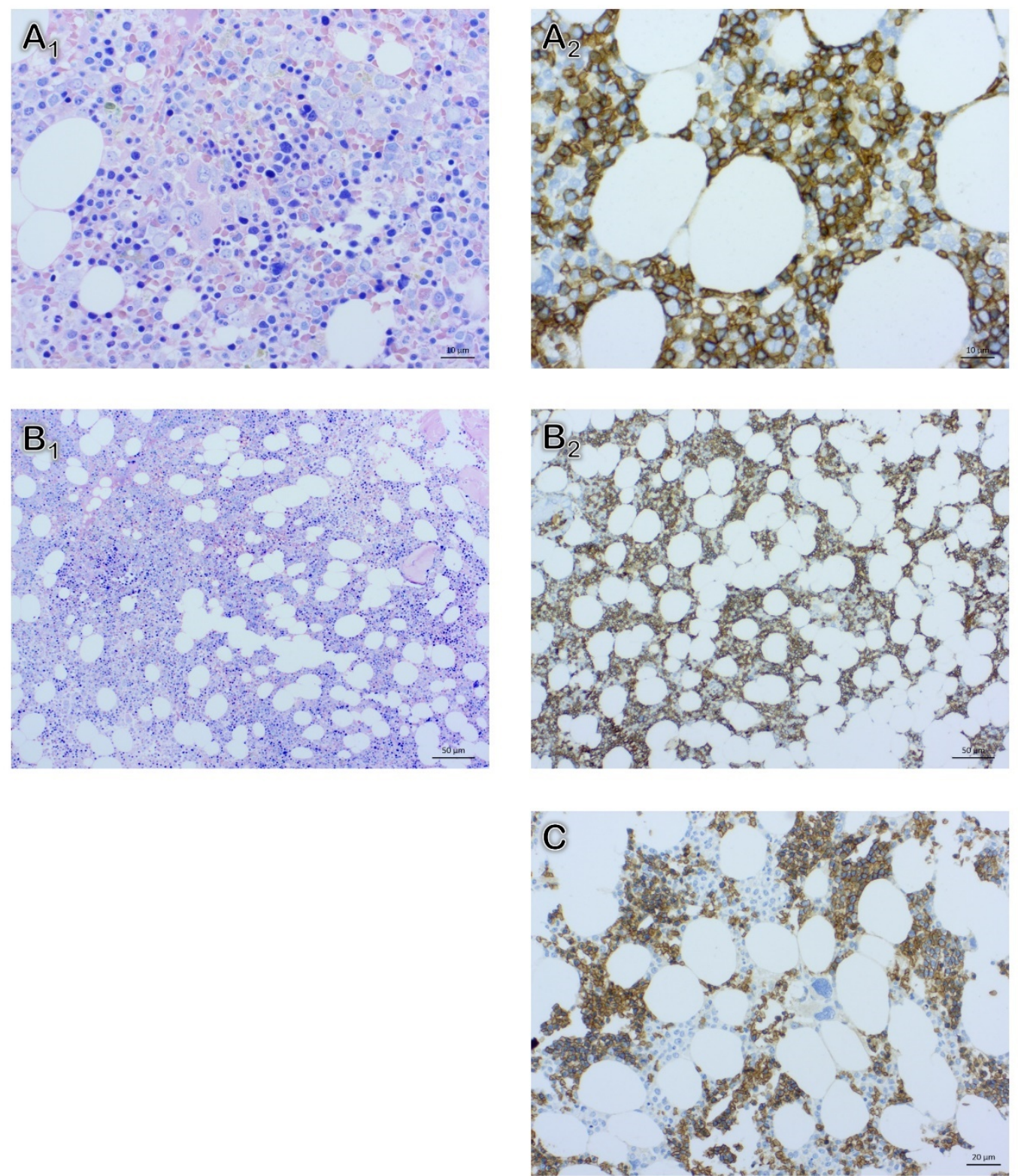

Abbildung 16: Dyserythropoiese

Veränderungen der Erythropoiese; A: Reifungsstörungen mit starken Unterschieden in der Kerngröße sowie zahlreichen makrozytotischen Vorläuferzellen [Patient ID-81, 40-fache Vergrößerung, $A_{1}$ : Giemsa-Färbung,

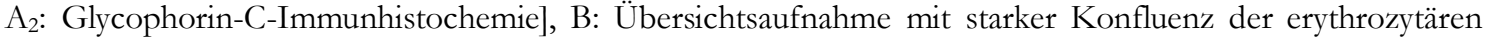
Nester [Patient ID-81, 10-fache Vergrößerung, $B_{1}$ : Giemsa-Färbung, B ${ }_{2}$ : Glycophorin-C-Immunhistochemie], C: mäßige Konfluenz der erythrozytären Nester [Patient ID-8, Glycophorin-C-Immunhistochemie, 20-fache Vergrößerung]; Quelle: eigene Präparate 


\subsubsection{Reifungs- und Architekturstörung der Megakaryopoiese}

Zur Beurteilung der megakaryozytären Vorstufen wurden ebenfalls Reifungs- und Architekturstörungen untersucht. Als morphologisches Korrelat einer gestörten Zellentwicklung wurde zuerst die verminderte Lappung der megakaryozytären Kerne (Hypolobulierung) beurteilt. Ebenfalls ein Hinweis auf eine Dysplasie dieser Zellreihe ist das Vorhandensein von kleinen (mikrozytären) Zellen (Abbildung 17). Ein drittes Anzeichen einer Reifungsstörung ist die Expression von CD34-Oberflächenantigenen in reifen Megakaryozyten (Abbildung 18). Diese ist eigentlich Kennzeichen unreifer (blastärer) Vorstufen der myeloischen Reihe.

Üblicherweise liegen Megakaryozyten im Knochenmark vereinzelt an der Wand von Sinusoiden. Ein Zeichen für eine Störung der Gewebsarchitektur ist die Entstehung von Gruppen (Clustern) von mehr als 3 Megakaryozyten (Wilkins und Porwit 2011) (Abbildung 17C). 

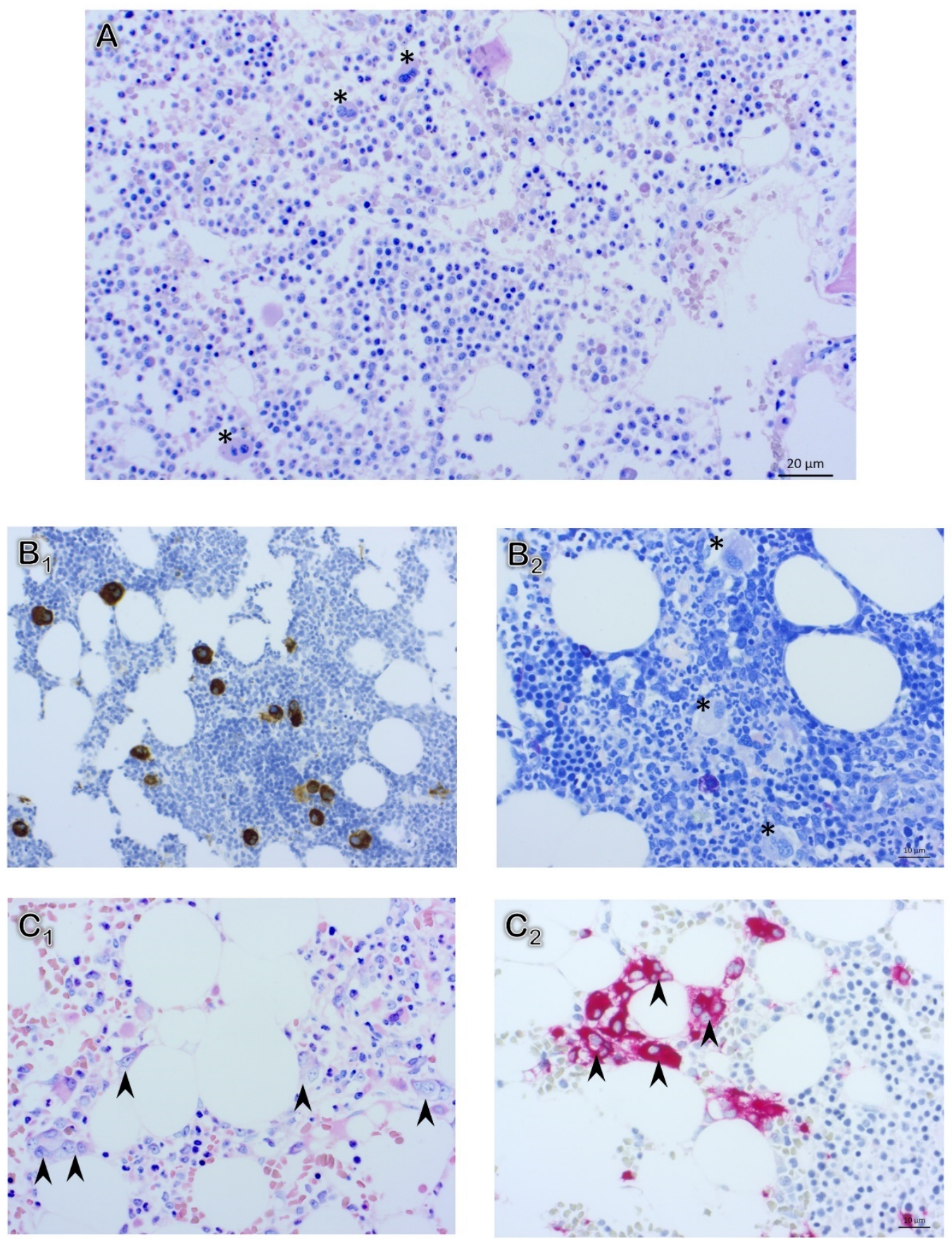

Abbildung 17: Dysmegakaryopoese

Veränderungen der Megakaryopoiese: A-B: ausgeprägte Mikrozytose und Hypolobulierung der Megakaryozyten, deutlich verminderte Kern-Plasma-Relation (*) (A: Patient ID-27, Giemsa-Färbung, 20-fache Vergrößerung, B: Patient ID-91, B B: CD-61-Immunhistochemie, 20-fache Vergrößerung, B ${ }_{2}$ : Giemsa-Färbung, 40-fache Vergrößerung), C: starke Clusterbildung mit Zusammenlagerung der atypisch ausreifenden Megakaryozyten ( $\bullet)$ (C: Patienten-ID 46, 40-fache Vergrößerung, $\mathrm{C}_{1}$ : Giemsa-Färbung, $\mathrm{C}_{2}$ : CD-61-Immunhistochemie-Färbung); Quelle: eigene Präparate 

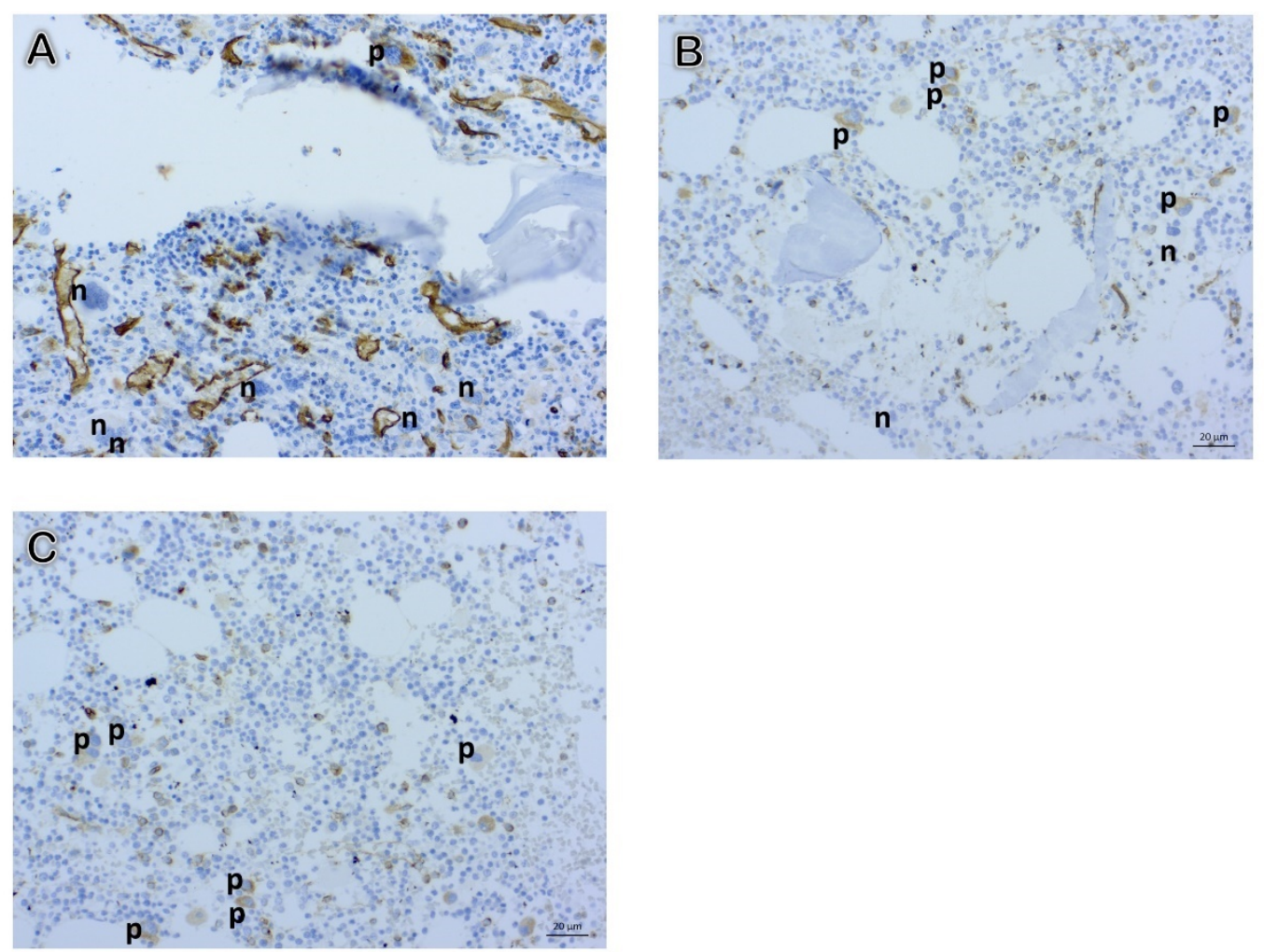

Abbildung 18: CD34-Expression auf Megakaryozyten

CD34-Expession der Megakaryopoiese: A: vereinzelt CD34+-Megakaryozyten (p), jedoch hauptsächlich negativ (n) [Patient ID-82, CD34-Immunhistochemie, 20-fache Vergrößerung], B: Teilweise CD34+ [Patient ID-73, CD34-Immunhistochemie, 20-fache Vergrößerung], C: alle Megakaryozyten sind CD34+ ${ }^{+}$Patienten-ID-46, CD34-Immunhistochemie, 20-fachen Vergrößerung]; Quelle: eigene Präparate

\subsubsection{Reifungs- und Architekturstörung der Granulopoiese}

Reifungsstörungen der Zellen der Granulopoiese wurden durch die Charakteristika der Linksverschiebung und der Zellgrößenvariabilität quantifiziert. Die Linksverschiebung beschreibt ein vermehrtes Vorkommen unreifer granulozytärer Vorstufen, wie Promyleozyten oder Metamyelozyten, sowie eine Verminderung reifer Vorstufen, wie stabkerniger- oder segmentkerniger Granulozyten. Für eine Störung der Zellausreifung sprechen auch Zellgrößenunterschiede, also die Verschiebung von gleichmäßig großen Zellen hin zu Zellen unterschiedlicher Größe.

Im normalen Knochenmark befinden sich die Zellen der granulozytären Vorstufen meist als Saum im Bereich der Knochenspongiosa. Ist diese normale Architektur aufgelöst, sodass sich Myeloblasten und Promyelozyten auch in zentralen Knochenmarksbereichen befinden, spricht man von ALIP (abnormal localization of immature precursors) (Tricot et al. 1984a; Tricot et al. 1984b). ALIP $^{+}$war ein Patient, wenn mindestens 3 Aggregate (3 - 5 Zellen) oder Cluster (> 5 Zellen) dieser Vorläuferzellen vorlagen. Diese Veränderungen sind in Abbildung 19 dargestellt. 

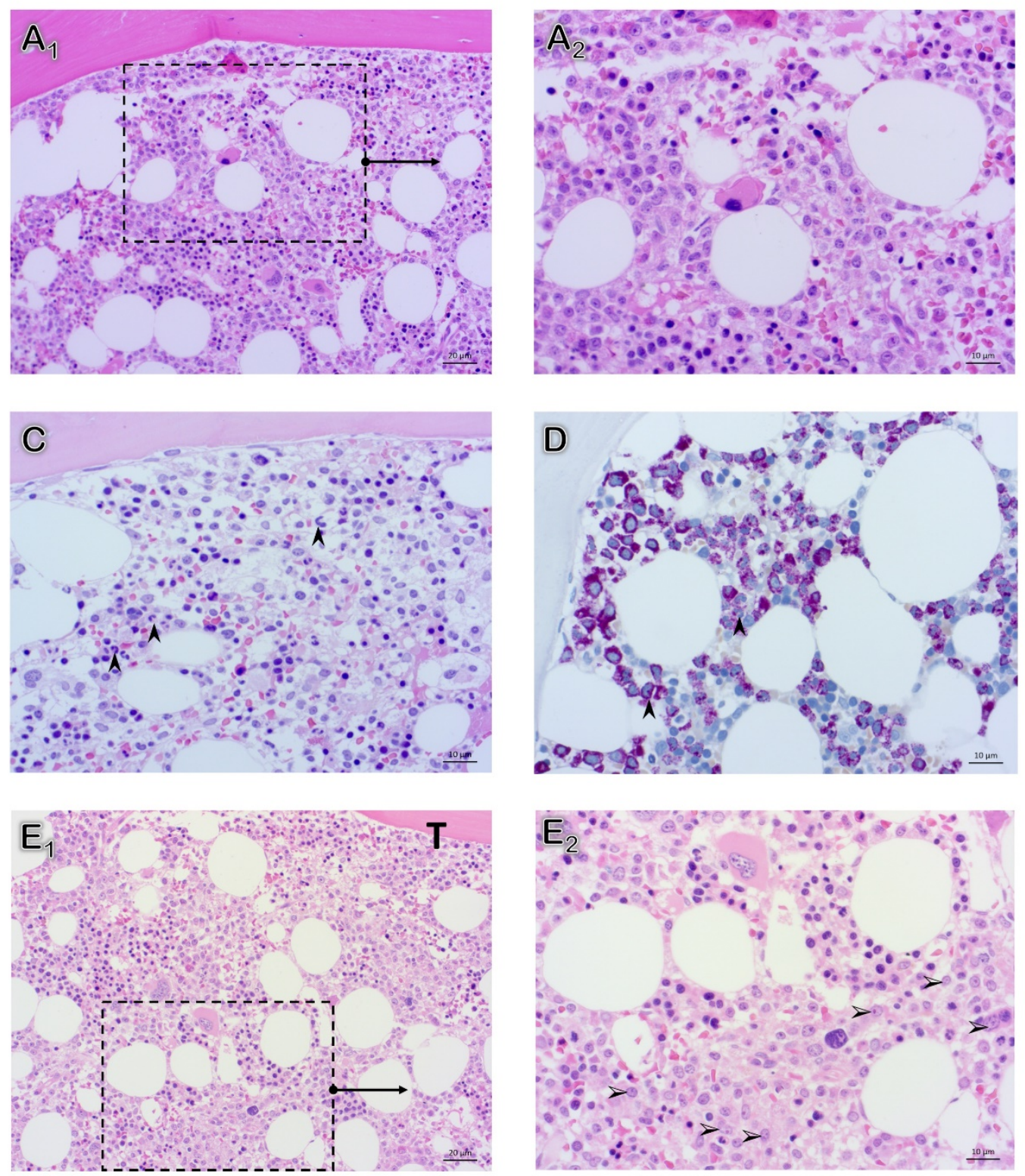

Abbildung 19: Dysgranulopoiese

Veränderungen der Granulopoiese: A: starke Linksverschiebung der Granulopoiese ohne reife Granulozyten [A: Patient ID-4, Hämatoxylin-Eosin-Färbung, $A_{1}$ : 20-fache Vergrößerung, $A_{2}$ : 40-fache Vergrößerung], C-D: mäßige Linksverschiebung mit noch einzelnen ausreifenden Formen ( $)$ [C: Patient ID-89, Hämatoxylin-EosinFärbung, 40-fache Vergrößerung, D: Patienten-ID 90, CAE-Färbung, 40-fache Vergrößerung], E: abnormal localization of immature precursors (ALIP) mit dem Vorkommen unreifer Vorstufen (Myeloblasten/Promyelozyten: $\triangleright$ ) entfernt von den Trabekeln (T) in der Markraummitte [E: Patient-ID 8, Hämatoxylin-Eosin-Färbung, E 20-fache Vergrößerung, E: 40-fache Vergrößerung]; Quelle: eigene Präparate 


\subsection{Statistische Tests}

\subsubsection{Spearman-Rangkorrelation}

Mit einer Korrelationsanalyse wurde der Zusammenhang zwischen zwei Variablen untersucht. Die in dieser Studie bei der histopathologischen Bewertung erhobenen Daten waren ordinal skalierte Ratingmerkmale (Kategorien in Reihenfolge der Merkmalsausprägung). Bei diesem Skalenniveau misst der Rangkorrelationskoeffizient nach Spearman einen monotonen Zusammenhang. Das heißt, mit sich verändernden Werten einer Variable ändert sich auch die andere Variable (Schneider et al. 2010). Da die histopathologischen Merkmale häufig eine schiefe (Nicht-Gaußssche) Verteilung zeigen, war er ebenfalls gut geeignet. Positive Vorzeichen des Koeffizienten zeigen einen positiven Zusammenhang an, negative Vorzeichen einen negativen. Je näher der Betrag des Koeffizienten an 1 heranreicht, desto stärker ist die Korrelation (Du Prel et al. 2010). Für die einzelnen Rangkorrelationskoeffizienten konnte die Signifikanz errechnet werden. Die Nullhypothese, dass kein Zusammenhang vorliegt, wurde getestet (Du Prel et al. 2010). In der vorliegenden Studie wurde ein Signifikanzniveau von $\alpha=0,05$ gewählt. Die Spearman-Rangkorrelation sowie die deskriptive Statistik wurde mit der Software STAISTICA [Dell Inc. (2015). Dell Statistica (Software-System für Datenanalyse), Version 12] durchgeführt.

\subsubsection{Regressionsanalysen}

Ein weiteres Verfahren, das für die Bestimmung eines Zusammenhangs genutzt wurde, war die Regressionsanalyse. Sie beschreibt ,inwiefern die Ausprägungen einer abhängigen Variablen sich durch die Kenntnis der Ausprägungen einer oder mehrerer unabhängiger Variablen schätzen lassen“ (Muche 2008).

Bei der linearen Regression kann anhand der entstehenden Formel (Regressionsgleichung) im Vergleich zur Rangkorrelation nicht nur die Richtung und Stärke des Zusammenhangs bestimmen, sondern auch wie dieser in Bezug auf die Einheiten quantifiziert wird. Der Regressionskoeffizient (b*, mathematisch die Steigung der Regressionsfunktion) gibt an, um welche Einheit die Zielvariable (hier IPSS-R) zunimmt, wenn sich die freie Variable um eine Einheit (hier morphologische Kategorien) erhöht (Schneider et al. 2010). Im Zusammenhang mit dem in dieser Dissertation als Zielvariable verwendetem IPSS-R ist die Interpretation der Ergebnisse allerdings stark eingeschränkt. Die Eigenschaften dieser Zielvariable widersprechen zwei Hauptannahmen des linearen Modells: Die Residuen sind nicht normalverteilt und sie ist nicht stetig, sondern kategorial skaliert (Gaus und Muche 2014). 
Deshalb wurde für die statistische Auswertung die ordinale logistische Regression für die Beurteilung von ordinal skalierten Ratingmerkmalen wie dem IPSS-R verwendet. Bei dieser wird nicht mehr eine Gleichung zur Beschreibung des Zusammenhangs modelliert, sondern die Wahrscheinlichkeit für das Eintreten der Zielergebnisses abhängig von den Zielvariablen (Muche 2008). Die in dem Modell errechneten Regressionskoeffizienten lassen sich in Odds Ratios (OR) überführen. Diese geben bei OR $>1$ an, dass eine Zunahme der Prädiktorvariablen auch eine Zunahme der Zielvariablen (hier IPSS-R) wahrscheinlicher macht. Wie auch bei der linearen Regression kann durch Einschluss von mehreren möglichen Einfluss- oder Störgrößen (Geschlecht, Alter) für deren Effekt adjustiert werden (Muche 2008). Auch bei der Regressionsanalyse ist eine Testung der statistischen Signifikanz möglich. Hierbei sind die p-Werte $<0,1,<0,05$ sowie $<0,01$ maßgeblich und werden in den entsprechenden Ergebnistabellen gekennzeichnet. Als Maß für die Stärke des Zusammenhangs dienen die Odds Ratios, die mit einem Konfidenzintervall von $95 \%$ angegeben werden (95\%-KI). In der vorliegenden Arbeit war es bei manchen Variablen sinnvoll diese als intervallskaliert zu kodieren, etwa beim Fibrosegrad oder ALIP. Bei quantitativen Veränderungen wurde hingegen eine Referenzgruppe aus Patienten mit normaler Zellzahl gebildet, mit der andere Gruppen (Verminderung, Vermehrung) verglichen wurden. Die Regressionsanalysen wurden mit der Software SPSS [BM Corp. Released 2013. IBM SPSS Statistics for Windows, Version 22.0. Armonk, NY: IBM Corp] durchgeführt. 


\section{Ergebnisse}

Im Rahmen der folgenden Beschreibung der Ergebnisse wird zuerst die beschreibende und folgernde Statistik des Gesamtkollektivs vorgestellt, danach die des Score-Kollektivs. Als primärer Endpunkt jeder induktiven Statistik wurde die zytogenetische Risikogruppe nach IPSS-R verwendet. Sekundäre Endpunkte waren die Klongröße, das Vorkommen komplexer Aberrationen und Veränderungen der Laborparameter.

\subsection{Gesamtkollektiv}

\subsubsection{Deskriptive Statistik}

\subsubsection{Patientenkollektiv}

In die Studie wurden 91 Patienten eingeschlossen. Von diesen waren 54 männlich (59 \%) und 37 weiblich (41\%). Das durchschnittliche Alter betrug $68 \mathrm{Jahre}(\operatorname{Mdn}=71 ; \operatorname{Min}=29$; Max $=85 ; \mathrm{SD}=10,7)$. In Abbildung 20 ist die Altersverteilung dargestellt. Sie zeigte einen ab dem 40. Lebensjahr kontinuierlichen Anstieg bis zu einem Maximum zwischen dem 70.75. Lebensjahr.

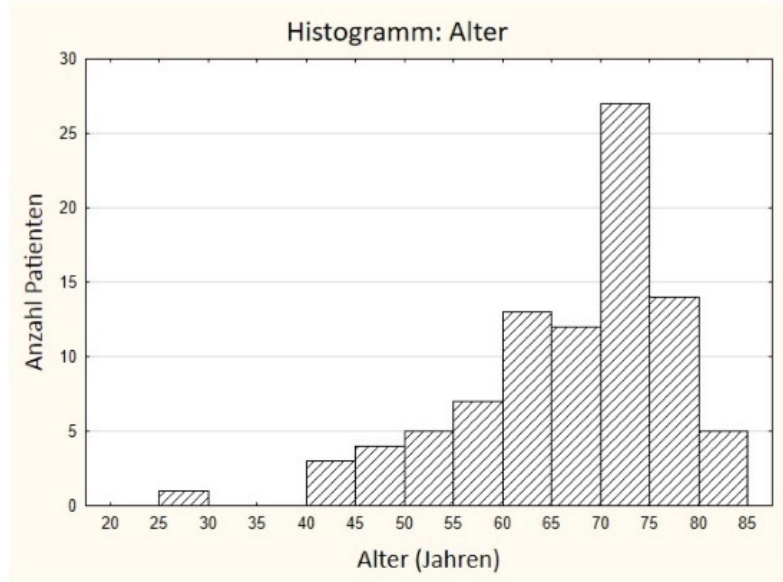

Abbildung 20: Altersverteilung im Gesamtkollektiv

\subsubsection{Laborwerte}

Die Patienten zeigten ein durchschnittlichen Hämoglobinwert von 9,6 mg/dl $(\mathrm{Mdn}=9,7$; $\operatorname{Min}=6,3 ; \operatorname{Max}=14,5 ; \mathrm{SD}=1,7)$, eine klinisch relevante Anämie mit einem Hämoglobinwert unter $10 \mathrm{mg} / \mathrm{dl}$ hatten 55 Patienten (60\%). Der Hämatokrit betrug im Schnitt 29,0 \% $(\operatorname{Mdn}=29 ; \operatorname{Min}=11,8 ; \operatorname{Max}=42,2 ; \mathrm{SD}=5,2)$. Die Erythrozytenzahl betrug durchschnittlich 3,19*10\% $/ \mu \mathrm{l}\left(\operatorname{Mdn}=3,14 * 10^{6} / \mu \mathrm{l}, \operatorname{Min}=2,06 * 10^{6} / \mu \mathrm{l} \operatorname{Max}=4,86 * 10^{6} / \mu \mathrm{l}\right.$; $\left.\mathrm{SD}=* 10^{6} / \mu \mathrm{l}\right)$. Der Durchschnitt der Plättchenzahl lag bei $119 * 10^{3} / \mu \mathrm{l}\left(\mathrm{Mdn}=81,40^{*}\right.$ 
$\left.10^{3} / \mu \mathrm{l} ; \operatorname{Min}=8 * 10^{3} / \mu \mathrm{l} ; \operatorname{Max}=671 * 10^{3} / \mu \mathrm{l} ; \mathrm{SD}=122 * 10^{3} / \mu \mathrm{l}\right)$. Eine nach WHO-Kriterien relevante Thrombozytopenie mit einer Plättchenzahl unter $100 * 10^{3}$ hatten 51 Patienten (56

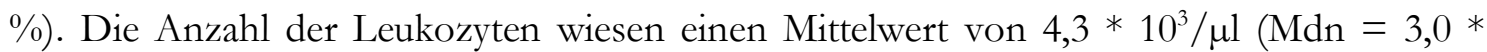
$\left.10^{3} / \mu \mathrm{l} ; \operatorname{Min}=0,4 * 10^{3} / \mu \mathrm{l} ; \operatorname{Max}=20,0 * 10^{3} / \mu \mathrm{l} ; \mathrm{SD}=4,2 * 10^{3} / \mu \mathrm{l}\right)$ auf. Eine Leukopenie mit Leukozytenzahlen unter $4,0 * 10^{3} / \mu$ l hatten 62 Patienten $(68 \%)$. Beim Vergleich der Laborwerte aus Gesamt- und Score-Kollektiv wurden die Werte gegenübergestellt (Tabelle 15).

\subsubsection{Genetische Veränderungen}

Von den 91 Studienpatienten zeigten 44 eine mittels Karyogramm oder FISH nachweisbare zytogenetische Veränderung (48 \%). Diese Aberrationen wurden anhand des IPSS-R in verschiedene zytogenetische Risikogruppen eingeteilt und eine Punktzahl von 0 - 4 vergeben. Der Gruppe „very good“ mit 0 Punkten wurde 1 Patient (1\%) zugeordnet, der Gruppe ,good“ (1 Punkt) 56 Patienten (62\%), ,intermediate“ (2 Punkte) 13 Patienten (14\%), ,poor“ (3 Punkte) 9 Patienten (10\%) sowie „very poor“ (4 Punkte) 12 Patienten (13\%). Patienten ohne zytogenetische Aberration werden in die Gruppe, good" einsortiert (Abbildung 21).

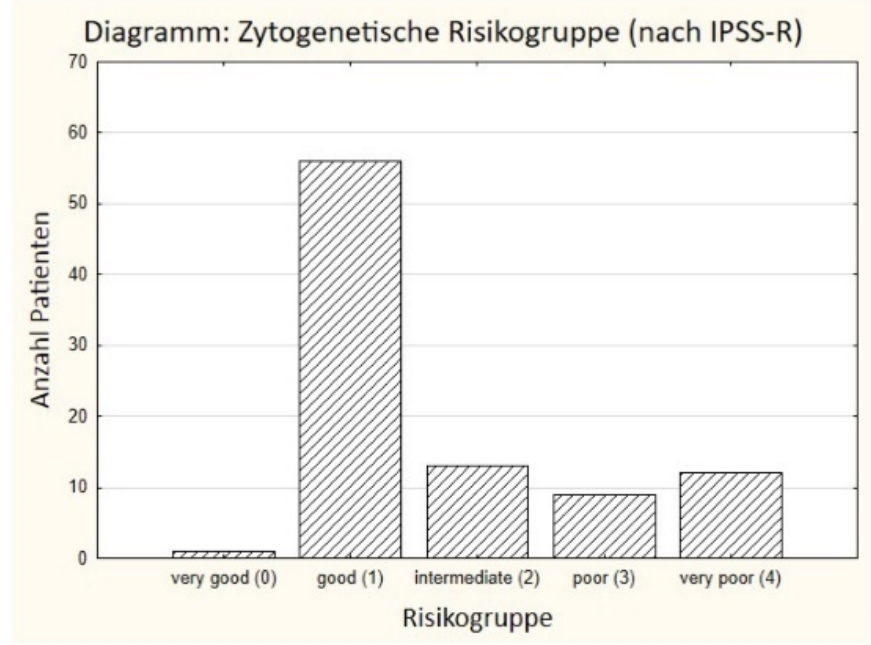

Abbildung 21: Zytogenetische Risikogruppen (nach IPSS-R)

Bei 79 Patienten lag eine Metaphasenuntersuchung zum Zeitpunkt der Knochenmarksbiopsie vor. Unterschiedliche Klongrößen konnten hier bestimmt werden. 13 Patienten (16\%) hatten ausschließlich abnorme Metaphasen, während bei 24 Patienten (30 \%) ein unterschiedlich ausgeprägtes Mosaik vorlag.

Die Anzahl der bewerteten Metaphasen war wichtig für die Aussagekraft. In Abbildung 22 sind diese dargestellt. Bei 60 Patienten (76\%) lag die Anzahl der untersuchten Metaphasen über 20 . 


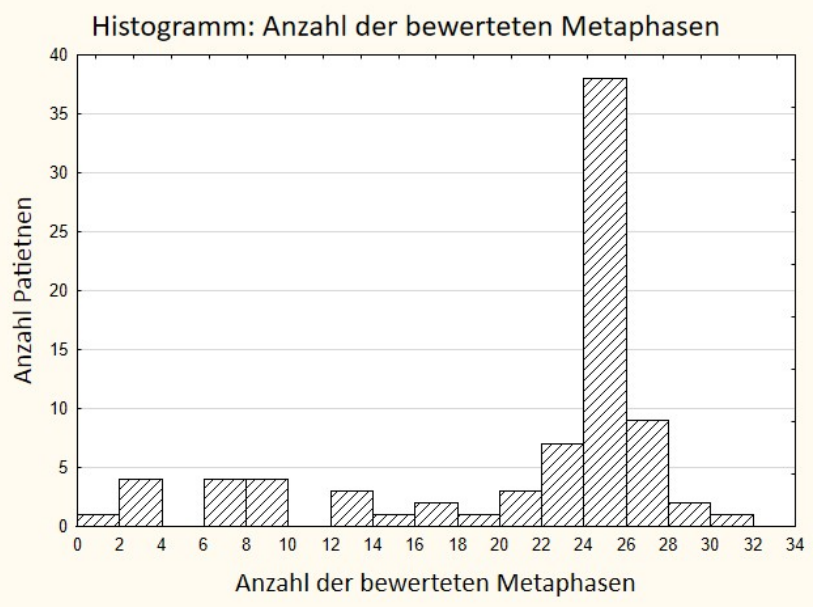

Abbildung 22: Anzahl der bewerteten Metaphasen im Gesamtkollektiv

Betrug die Anzahl der Metaphasen weniger als 15, war mit einer eingeschränkten Sensitivität der Untersuchung zu rechnen. Die Charakteristika dieser Patienten sind in Tabelle 8 aufgelistet.

Tabelle 8: Charakteristika von Patienten mit weniger als 15 Metaphasen

\begin{tabular}{|c|c|c|c|c|c|}
\hline ID & Genetik & MPG & MPA & $\begin{array}{l}\text { IPSS-R } \\
\text { Riskio }\end{array}$ & Klinische Angaben \\
\hline$* 2$ & $46, X X-7[6] / 46, X X, \operatorname{del}(7)(q 22)[3] / 46, X X[1]$ & 10 & 9 & 3 & v.a. MDS \\
\hline$* 7$ & $\begin{array}{l}\text { 47,X,der(Y;1)(q12;q12),+8 } \\
{[2] / 46, X Y, \operatorname{der}(1 ; 7)(p 10 ; q 10[6] / 46, X Y[5]}\end{array}$ & 13 & 8 & 2 & Verlaufkontrolle ohne Therapie \\
\hline$* 8$ & 46,XY, del(20)(q11.2q13.3) [2]/46,XY [1] & 3 & 2 & 1 & Verlaufkontrolle ohne Therapie \\
\hline 13 & $45, X X,-7[3]$ & 3 & 3 & 3 & Verlaufskontrolle unter Therapie \\
\hline *17 & $46, X, \operatorname{der}(Y) t(Y ; 1)(q 12 ; q 12)[2] / 47$, idem, $+8[6]$ & 8 & 8 & 3 & v.a. MDS \\
\hline$* 18$ & $46, X Y, \operatorname{del}(20)(q 12)[1]$ & 1 & 1 & 1 & v.a. MDS \\
\hline 20 & $46, X X,[13]$ & 13 & 0 & 1 & v.a. MDS \\
\hline 23 & $46, X X,[3]$ & 3 & 0 & 1 & v.a. MDS \\
\hline 31 & $46, X Y,[8]$ & 8 & 0 & 1 & Verlaufkontrolle ohne Therapie \\
\hline 43 & $46, X X,[9]$ & 9 & 0 & 1 & Verlaufkontrolle ohne Therapie \\
\hline$* 46$ & $47, \mathrm{XY}, \mathrm{t}(3 ; 3)(\mathrm{q} 21 ; \mathrm{q} 26),+\operatorname{mar}[6] / 46, \mathrm{XY}[1]$ & 8 & 7 & 2 & v.a. MDS \\
\hline 47 & $47, X Y, t(3 ; 3)(q 21 ; q 26),+\operatorname{mar}[6] / 46, X Y[1]$ & 8 & 7 & 2 & Verlaufskontrolle unter Therapie \\
\hline$* 48$ & Komplex & 10 & 8 & 4 & v.a. MDS \\
\hline$* 64$ & $46, X Y,[4]$ & 4 & 0 & 1 & v.a. MDS \\
\hline$* 69$ & $46, X Y,[14]$ & 14 & 0 & 1 & v.a. MDS \\
\hline
\end{tabular}

Legende: ID: Identifikationsnummer, MPG: Metaphasen gesamt, MPA: Metaphasen abnorm, v.a. MDS: Verdacht auf MDS; *: Patient auch im Score-Kollektiv enthalten

Bei 12 Patienten wurde das genetische Risiko vorwiegend anhand der FISH-Analyse ermittelt. Tabelle 9 zeigt, welche Patienten mit FISH-Analyse zum Zeitpunkt der KM-BiopsieEntnahme in die Studie eingeschlossen wurden. Im Falle der Pateinten ID24, ID25 und ID42 war die unauffällige Zytogenetik in vorausgegangenen Untersuchungen festgestellt worden. Für ID73 und ID79 lag ein späterer Befund mit der von der FISH festgestellten Genetik vor. Die Patienten ID79 und ID82 wiesen bereits in der pB- bzw. KM-FISH einen komplexaberranten Karyotyp auf. Ein ungünstigerer Karyotyp mit weiteren nachgewiesenen Aberrationen hätte hier nicht zu einer schlechteren Einordnung geführt. Die Patienten ID10 und 
ID19 wurden wegen der 17p- (p53) Deletion in die IPSS-R Gruppe 4 und nicht 3 eingeordnet, obwohl nur drei Veränderungen in der pB/KM-FISH nachgewiesen worden waren. Erfahrungsgemäß liegen bei dieser Aberration weitere, nicht detektierte Veränderungen vor. Bei den Patienten ID21 und ID80 war die unauffällige FISH die einzige durchgeführte genetische Analyse. Beim Patienten ID51 war der Karyogrammbefund normal und die FISHAnalyse auffällig. Letztere wurde in diesem Fall als wegweisend gewertet.

Tabelle 9: Bestimmung der Genetik mittels pB-/KM-FISH

\begin{tabular}{|c|c|c|c|c|}
\hline ID & Genetik & Bemerkung & $\begin{array}{l}\text { IPSS-R } \\
\text { Riskio }\end{array}$ & Klinische Angaben \\
\hline$* 10$ & Komplex (PB-FISH: 5q-, 7q, 17p-) & p53 & 4 & v.a. MDS \\
\hline 19 & Komplex (KM-FISH: 5q-, +8, 17p-) & p53 & 4 & Verlaufskontrolle unter Therapie \\
\hline 21 & $46, \mathrm{XY}$ (PB-FISH) & & 1 & Verlaufkontrolle ohne Therapie \\
\hline 24 & $46, \mathrm{XY}(\mathrm{PB}-\mathrm{FISH})$ & Vorbefunde & 1 & Verlaufkontrolle ohne Therapie \\
\hline 25 & $46, \mathrm{XY}(\mathrm{PB}-\mathrm{FISH})$ & Vorbefunde & 1 & Verlaufkontrolle ohne Therapie \\
\hline 42 & $46, X Y(P B-F I S H)$ & Vorbefunde & 1 & Verlaufkontrolle ohne Therapie \\
\hline 50 & Komplex (PB-FISH: 5q-, -7, +8, 13q-, 17p-, 20q-) & Maximum & 4 & Verlaufskontrolle unter Therapie \\
\hline$* 51$ & -7 (FISH; Karyogramm: 46, XX [9]) & Zusatzanomalie & 3 & v.a. MDS \\
\hline *73 & $5 q-(P B-F I S H)$ & Folgebefunde & 1 & v.a. MDS \\
\hline$* 79$ & 20q- (PB-FISH) & Folgebefunde & 1 & v.a. MDS \\
\hline$* 80$ & FISH: 7q- (PB-FISH) & & 2 & v.a. MDS \\
\hline *82 & Komplex (PB-FISH: 5q-, 7q-, 13q, 17p-) & Maximum & 4 & v.a. MDS \\
\hline
\end{tabular}

Legende: ID: Identifikationsnummer, v.a. MDS: Verdacht auf MDS; *: Patient auch im Score-Kollektiv enthalten

\subsubsection{Klinische Angaben}

Anlass für eine Knochenmarksbiopsie war bei 50 Patienten (55\%) der Verdacht auf ein myelodysplastisches Syndrom. Bei 25 Patienten (27\%) war ein MDS bereits bekannt, jedoch ohne Therapie, und 16 Patienten (18\%) wurden aktuell oder in der Vergangenheit mit Chemotherapie oder Stammzelltransplantation behandelt. Außerdem gab es bei 13 Patienten (14\%) Grund zur Annahme, dass ein sekundäres, Therapie-assoziiertes MDS bestand.

\subsubsection{Ergebnisse der Routinediagnostik}

In der ersten Durchsicht wurden alle 91 Präparate der normalen Diagnostik zur histopathologischen Einordnung bezüglich MDS unterzogen. Der alterskorrigierte Anteil der Hämatopoiese war das zuerst betrachtete Kriterium. Bei 8 Patienten (9\%) zeigte sich ein hypoplastisches Knochenmark. In den meisten Fällen waren die blutbildenden Zellen mäßig (35 Patienten, $38 \%$ ) oder stark (32 Patienten, 35\%) erhöht. Abbildung 23A zeigt die Verteilung der verschiedenen Hämatopoieseanteile. Eiseneinlagerungen im Knochenmark (Markraumsiderose) kamen bei den Patienten weitgehend gleichmäßig auf die verschiedenen Schweregrade $(0=$ normal bis $3=$ schwere Eiseneinlagerung) verteilt vor (Abbildung 23B). Eine Faservermehrung war in leichter (16 Patienten, $18 \%$ ) oder mäßiger (15 Patienten, $16 \%$ ) Form 
nachzuweisen, während die meisten Patienten keine gesteigerte extrazelluläre Matrix zeigten (60 Patienten, $66 \%$ ) (Abbildung 23C).

A

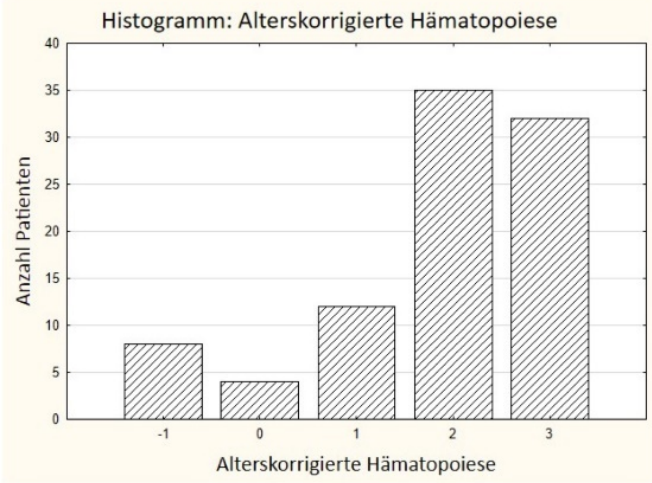

C

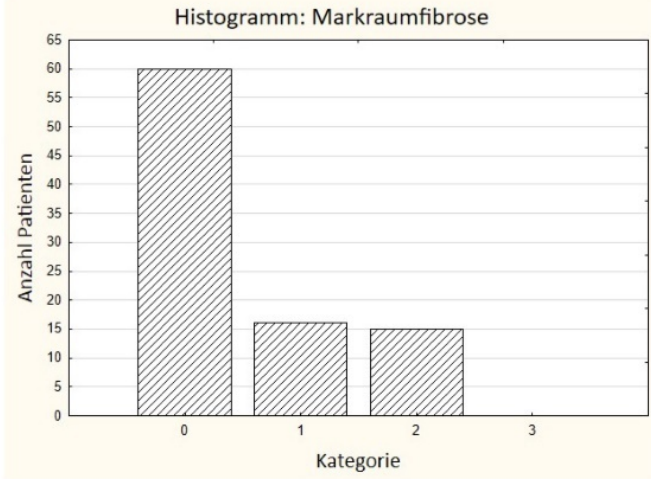

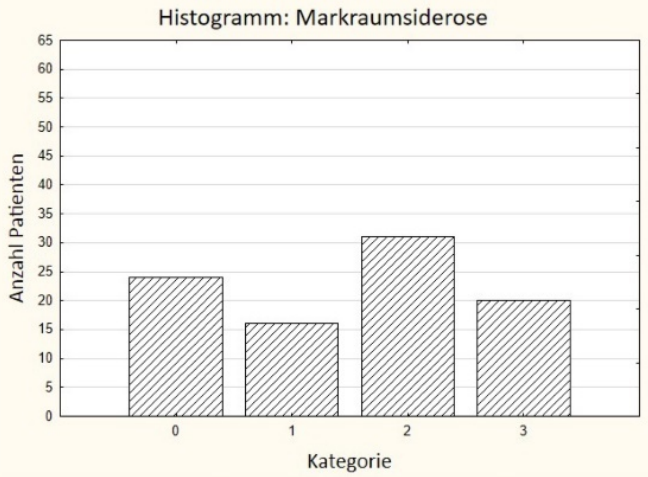

Abbildung 23: Morphologische Veränderungen der Routinediagnostik

A: Verteilung der alterskorrigierten Hämatopoiese (-1, 0, 1, 2, 3), B: Verteilung der Markraumsiderose (0-3), C: Verteilung der Markraumfibrose (0-3)

Ein weiteres histomorphologisches Kriterium in der Routinediagnostik ist das Vorkommen von Blasten im Knochenmark. Bei der Färbung des CD34-Antigens zeigten 28 Patienten (31 \%) einen normalen Blastenanteil von unter $2 \%$. Eine leichte Erhöhung $(\geq 2 \%$ und $<5$ \%) ließ sich bei 19 Pateinten (21\%) feststellen. Eine mäßige Blastenerhöhung und damit die WHO-Klassifikationsgruppe MDS-EB-1 war bei 19 Patienten (21\%) zu diagnostizieren. Stark erhöht (MDS-EB-2) waren die CD34+-Blasten bei 25 Patienten (26\%). Eine CD117Immunhistochemie wurde bei 58 Patienten durchgeführt. Von diesen wiesen 31 Patienten (53\%) einen Blastenanteil unter $2 \%$ auf, 9 Patienten (16\%) einen zwischen $2-4 \%, 6$ Patienten (10\%) zwischen 5 - $9 \%$ (MDS-EB-1) und 12 Patienten (21\%) einen Anteil über $10 \%$ (MDS-EB-2). Da nicht für alle Patienten eine Färbung mittels CD117Immunhistochemie durchgeführt wurde, wurde dieser Parameter nicht im Rahmen der Regressionsanalysen einbezogen. Fehlende Werte einer Variablen führen hier zu einem Ausschluss auch aller anderen Werte desselben Patienten. Bei einer Betrachtung der beiden Histogramme (Abbildung 24A/B) zeigte sich eine ähnliche Verteilung der beiden immunhistochemischen Marker. 
A

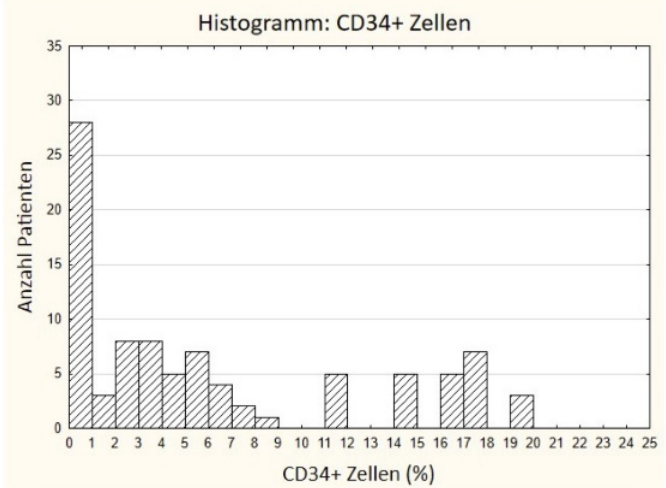

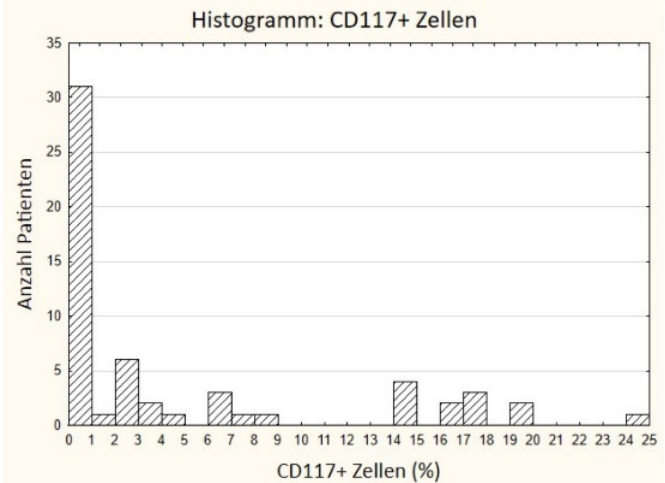

Abbildung 24: Immunhistochemie im Gesamtkollektiv A: CD34-Expression von Zellen, B: CD117-Expression von Zellen

Am Ende der Routinediagnostik steht die Einteilung in die WHO-Klassifikation. Bei 3 Patienten (3\%) wurde MDS-SLD diagnostiziert. Die größte Patientengruppe bildeten die an MDS-MLD Erkrankten mit 39 Patienten (43\%). Als MDS-EB-1 wurden Befunde von 20 Patienten (22\%) klassifiziert und als MDS-EB-2 die von 29 Patienten (32\%) (Abbildung 25).

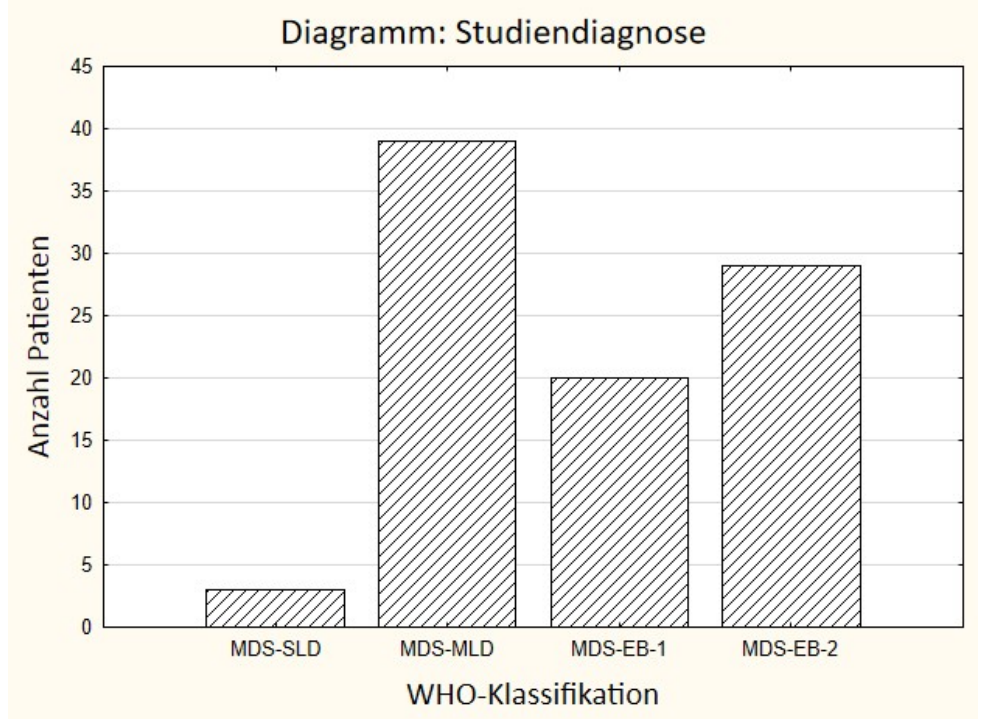

Abbildung 25: Diagnose nach WHO-Klassifikation

\subsubsection{Spearman-Rangkorrelation}

Um einen möglichen Zusammenhang zwischen den histopathologischen Routineparametern und dem zytogenetischen Risiko zu ermitteln, wurde eine Spearman-Rangkorrelation durchgeführt. Mit dem primären Endpunkt der genetischen Risikogruppe nach IPSS-R korrelierten die Siderose $(0,28)$, die Markraumfibrose $(0,40)$ sowie das Vorhandensein von CD $34^{+}-$ Zellen $(0,27)$ (Tabelle 10). Insgesamt betrachtet waren diese Korrelationen schwach. 
Bei Betrachtung der sekundären Endpunkte bestand ebenfalls ein Zusammenhang zwischen Fibrose/Siderose und einer erhöhten Klongröße (jeweils 0,28). Eine Korrelation zeigte sich auch zwischen histologischen Kriterien und Veränderungen im peripheren Blut. Diesbezüglich bestand ein Zusammenhang zwischen einer vermehrten Eiseneinlagerung und der Verminderung der roten Blutzellen (Hämoglobin: -0,22, Hämatokrit: -0,26; Erythrozyten: -0,27). Eine Fibrose des Markraums korrelierte negativ sowohl mit der Erythropoiese (Hämoglobin: -0,28; Hämatokrit: -0,32; Erythrozyten: -0,28) als auch der Thrombopoiese (Thrombozyten: $-0,22)$ und der Leukozytenzahl $(-0,21)$. Letztere war auch erhöht bei gesteigerter Hämatopoiese im Knochenmark $(0,22)$ und erniedrigt bei Lymphozyten im histologischen Präparat $(-0,34)$.

Tabelle 10: Spearman-Rangkorrelation von Parametern der ersten Durchsicht

\begin{tabular}{l|rrrrrrrrr}
\hline N=91 & Hb & HKT & Ery. & MCV & Plt. & Leu. & KG & Kom. IPSSR \\
\hline Alterskorr. Häm. &,- 10 &, 05 &, 17 & $*_{-, 27}$ &,- 08 & $*_{, 22}$ &, 10 & $*_{, 26}$ &, 13 \\
Lymphozyten &,- 16 &,- 14 &,- 15 &,- 11 &,- 13 & $*_{-}, 34$ &,- 08 &, 21 &, 08 \\
Siderose & $*_{-, 22}$ & $*_{-}, 26$ & $*_{-}, 27$ &, 06 &,- 03 &,- 12 & $*_{, 28}$ &, 15 & $*_{, 28}$ \\
Fibrose & $*_{-, 28}$ & $*_{-}, 32$ & $*_{-}, 28$ &,- 08 & $*_{-, 22}$ & $*_{-}, 21$ & $*_{, 28}$ & $*_{, 25}$ & $*_{, 40}$ \\
CD34+-Zellen &, 03 &,- 06 &,- 07 &, 11 &,- 13 &,- 13 &, 03 &, 17 & $*_{, 27}$ \\
CD117+-Zellen &, 01 &, 01 &,- 07 &,- 02 &, 06 &,- 01 &,- 11 &, 24 &, 17 \\
\hline \hline
\end{tabular}

Legende: Hb: Hämoglobin HKT: Hämatokrit, Ery.: Erythrozyten, MCV: mean corpuscular volume, Plt.: Plättchen, Leu.: Leukozyten, KG: Klongröße, Kom.: Komplex, Alterskorr. Häm.: alterskorrigierte Hämatopoiese, * $\mathrm{p}<0,05$

\subsubsection{WHO-Klassifikation und Zytogenetik}

Nach der Betrachtung der einzelnen histopathologischen Routineparameter und ihrem Zusammenhang zum IPSS-R wurde untersucht, inwiefern die abschließende Diagnose nach WHO-Klassifikation mit dem zytogenetischen Risiko in Verbindung stand. Abbildung 26 stellt die absolute und relative Verteilung der IPSS-R-Risikogruppen innerhalb der WHOKategorien dar. MDS-MLD-Patienten mit der genetischen IPSS-R-Risikogruppe 1 Punkt (,good $\left.{ }^{*}\right)$ stellten die meisten Patienten dar, weil Patienten mit negativer Zytogenetik hier eingeordnet wurden. Bei Betrachtung der relativen Häufigkeiten zeigte sich eine ähnliche Verteilung der IPSS-R Risikogruppen für Genetik auf die WHO-Subtypen. Der Anteil der ungünstigeren Zytogenetik schien im Verlauf moderat zuzunehmen. 

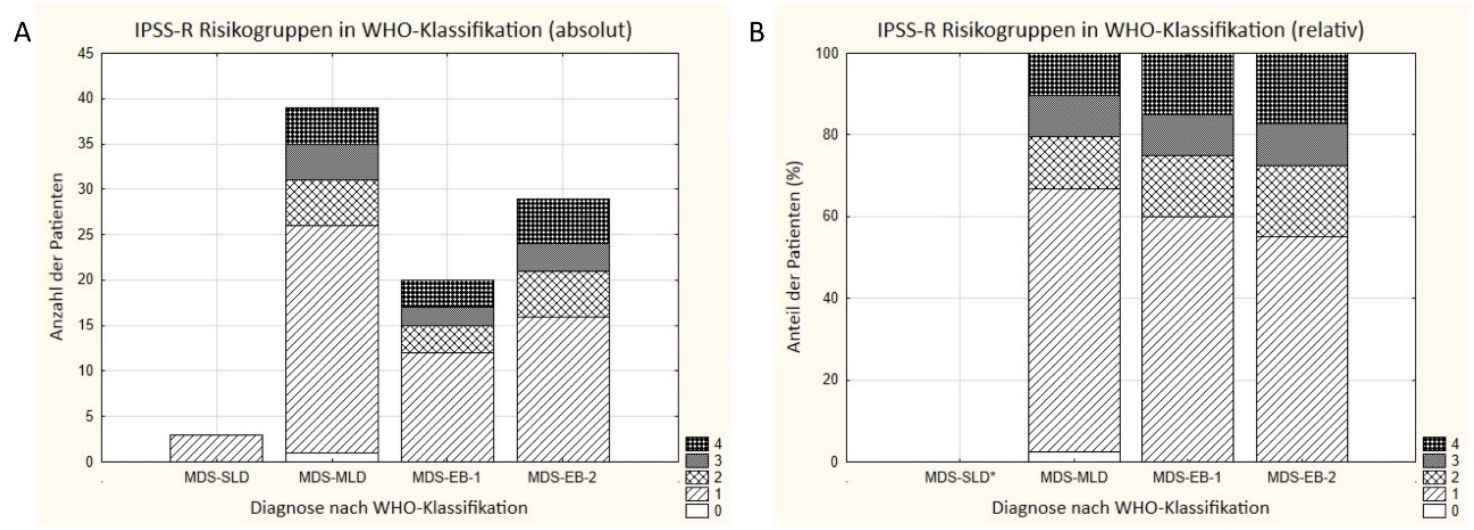

Abbildung 26: IPSS-R Risikogruppen in der WHO-Klassifikation Gesamt-Kollektiv A: Darstellung der absoluten Häufigkeiten von zytogenetischen Risikogruppen je WHO-Kategorie; B: Darstellung der relativen Häufigkeiten von zytogenetischen Risikogruppen je WHO-Kategorie; *aufgrund der geringen Anzahl dieser Merkmalsausprägung, wurden die relativen Werte (100\% genetische IPSS-R-Gruppe 1) nicht dargestellt

Zur Untersuchung eines Zusammenhangs wurde eine Spearman-Rangkorrelation durchgeführt und die WHO-Kategorien mit aufsteigenden Nummern (MDS-SLD =1, MDS-MLD $=2$, MDS-EB-1 $=3$ und MDS-EB-2 = 4) kodiert und mit dem IPSS-R korreliert. Hierbei zeigte sich keine signifikante Korrelation (Rangkorrelationskoeffizient: 0,16, p-Wert >0,05). Der Einfluss der WHO-Klassifikation auf das genetische Risiko wurde zusätzlich mit einer Regressionsanalyse untersucht. Ein Zusammenhang konnte nicht nachgewiesen werden (Tabelle 11).

Tabelle 11: Regression des IPSS-R mit dem Prädiktor WHO-Subtyp im Gesamtkollektiv

\begin{tabular}{lrc}
\hline $\mathrm{N}=91$ & $\mathrm{p}$-Wert & Odds Ratio $95 \%-\mathrm{KI}$ \\
\hline WHO-Subtyp & & - \\
MDS-MLD (Ref.) & \multicolumn{2}{c}{-} \\
MDS-EB-1 & 0,52 & $1,42[0,49 ; 4,16]$ \\
MDS-EB-2 & 0,27 & $1,70[0,66 ; 4,38]$ \\
\hline \hline
\end{tabular}




\subsubsection{Regressionsanalyse im Gesamtkollektiv}

Bei der univariaten ordinalen Regression bestätigte sich das Ergebnis der Korrelationsanalyse: Markraumfibrose, Markraumsiderose und der Anteil der CD $34^{+}$-Zellen hatten einen Einfluss auf das genetische Risiko (Tabelle 12). Hierbei war jedoch der potentielle Einfluss der $\mathrm{CD}_{3} 4^{+}$mit einem OR von 1,07 und einem 95\%-KI von 1,00-1,14 relativ gering.

Tabelle 12: Univariate Regression des IPPS-R mit Prädiktoren der Routineparameter

\begin{tabular}{lcc}
\hline $\mathrm{N}=91$ & $\mathrm{p}$-Wert & Odds Ratio 95\% -KI \\
\hline Alterskorrigierte Hämatopoiese & & \\
\hline $0 / 1$ (Ref.) & & - \\
-1 & 0,34 & $2,28[0,42 ; 12,38]$ \\
+2 & 0,43 & $1,72[0,45 ; 6,63]$ \\
+3 & 0,13 & $2,87[0,74 ; 11,16]$ \\
\hline
\end{tabular}

\begin{tabular}{lcr}
\hline $\begin{array}{l}\text { Lymphozyten } \\
\text { Nein (Ref.) }\end{array}$ & \multicolumn{2}{c}{. } \\
$\mathrm{Ja}$ & 0,49 & $1,43[0,57 ; 3,57]$ \\
\hline Eisen & 0,01 & $* * 1,81[1,19 ; 2,74]$ \\
\hline Fibrose & $<0,01$ & $* * * 2,81[1,60 ; 4,94]$ \\
\hline CD34 ${ }^{+}$-Zellen & 0,03 & $* * 1,07[1,00 ; 1,14]$ \\
\hline \hline & Legende: Ref.: Referenzkategorie, ${ }^{* * *} \mathrm{p}<0,01 ; * * \mathrm{p}<0,05 ;{ }^{*} \mathrm{p}<0,1$
\end{tabular}

In Tabelle 13 ist die ordinale logistische Regression des IPSS-R im Gesamtkollektiv unter Einschluss möglicher Störvariablen (Ätiologie, Alter, Geschlecht) dargestellt. Es zeigt sich, dass insbesondere die zunehmende Fibrose mit einem starken und hochsignifikanten Risiko für eine Steigerung des IPSS-R einhergeht. Ein Trend lässt sich weiterhin erkennen für einen Zusammenhang zwischen der Siderose des Markraums und einem gesteigerten Risiko für eine ungünstige Genetik: das Konfidenzintervall schließt die 1 nur knapp mit ein und das potentiell vorhandene Risiko ist deutlich (Obere Grenze des 95\%-KI bei 2,66). Der in der univariaten Regression gezeigte Einfluss des Anteils CD34+-Zellen ist nicht mehr nachzuweisen. Adjustiert sind diese Ergebnisse für den Einfluss des Alters sowie der Ätiologie, die ebenfalls signifikant waren in diesem Modell. 
Tabelle 13: Ordinale logistische Regression des IPPS-R im Gesamtkollektiv

\begin{tabular}{|c|c|c|}
\hline $\mathrm{N}=91$ & p-Wert & Odds Ratio \\
\hline \multicolumn{3}{|c|}{ Alterskorrigierte Hämatopoiese } \\
\hline 0/1 (Ref.) & & - \\
\hline-1 & 0,92 & $0,90[0,11 ; 7,43]$ \\
\hline+2 & 0,26 & $2,62[0,49 ; 14,11]$ \\
\hline+3 & 0,16 & $2,98[0,64 ; 13,88]$ \\
\hline \multicolumn{3}{|l|}{ Lymphozyten } \\
\hline Nein (Ref.) & & - \\
\hline $\mathrm{Ja}$ & 0,85 & $1,13[0,32 ; 3,95]$ \\
\hline Siderose & 0,09 & $* 1,58[0,93 ; 2,66]$ \\
\hline Fibrose & $<0,01$ & $* * * 2,79[1,48 ; 5,26]$ \\
\hline CD34+-Zellen & 0,31 & $1,04[0,96 ; 1,12]$ \\
\hline \multicolumn{3}{|l|}{ Ätiologie } \\
\hline Primär (Ref.) & & - \\
\hline Sekundär & 0,02 & $* * 5,47[1,31 ; 22,87]$ \\
\hline Alter & 0,09 & $* 0,96[0,92 ; 1,01]$ \\
\hline \multicolumn{3}{|l|}{ Geschlecht } \\
\hline Männlich (Ref.) & & - \\
\hline Weiblich & 0,12 & $0,42[0,14 ; 1,24]$ \\
\hline
\end{tabular}

Legende: Ref.: Referenzkategorie, ${ }^{* * *} \mathrm{p}<0,01$; ** $\mathrm{p}<0,05$; ${ }^{*} \mathrm{p}<0,1$

\subsection{Score-Kollektiv}

Die prognostische Relevanz von morphologischen Veränderungen im Knochenmark bei Patienten mit MDS, die über die Routineparameter hinausgehen, sollte bestimmt werden. Ein Kollektiv aus Patienten mit einem sicheren MDS wurde gebildet. Ausgeschlossen von der weiteren Analyse wurden Patienten, bei denen weder eine zytogenetische Aberration noch eine Vermehrung von Blasten im Knochenmark ( $\mathrm{um}>5 \%$ ) nachgewiesen werden konnte. Um den Einfluss begleitender Therapien auf die Morphologie der Zellen zu vermeiden, wurden Patienten nach oder unter Therapie ebenfalls ausgeschlossen. Zuerst wird die Zusammensetzung der beiden Kollektive verglichen und anschließend werden die Ergebnisse des Morphologie-Scores beschrieben.

\subsubsection{Vergleich von Gesamt- und Score-Kollektiv}

Das Score-Kollektiv bestand aus 55 Patienten (60\% des Gesamtkollektivs). Ein Vergleich beider Gruppen zeigte eine weitgehende Übereinstimmung. Im neuen war der Frauenanteil kleiner (33\% vs. $41 \%$ ). Die Altersverteilung sowie die Laborparameter waren in beiden 
Gruppen vergleichbar, wobei im zweiten Kollektiv die Plättchen- und Leukozytenzahl im Durchschnitt etwas niedriger lag.

Durch den Ausschluss von Patienten haben sich möglicherweise die Eigenschaften in Bezug auf bestimmte Variablen verändert. Um dies auszuschließen, wurden die beiden Kollektive miteinander verglichen. Für die kategorialen Variablen wurde ein t-Test verwendet (Tabelle 14), für metrische Variablen der exakte Test nach Fisher (Tabelle 15). Es zeigte sich kein signifikanter Unterschied zwischen Gesamt- und Score-Kollektiv in Bezug auf das Alter, Geschlecht, die Laborparameter und - für die Interpretation besonders relevant - beim Zielparameter IPSS-R. Ein signifikanter Unterschied trat in Bezug auf die Verteilung der verschiedenen WHO-Subtypen auf, bedingt durch den Ausschluss vieler MDS-MLD-Patienten (keine Blastenerhöhung und oft keine zytogenetischen Veränderungen).

Tabelle 14: Exakter Test nach Fisher zum Vergleich von Anteilen

\begin{tabular}{lccr}
\hline & Gesamtkollektiv & Score-Kollektiv & \\
\hline Geschlecht & $\mathrm{N}($ Anteil $)$ & $\mathrm{N}($ Anteil $)$ & $\mathrm{p}$-Wert \\
Männlich & $54(59,3 \%)$ & $37(67,3 \%)$ & \\
Weiblich & $37(40,7 \%)$ & $18(32,7 \%)$ & 0,34 \\
\hline IPSS-R & & & \\
0 & $1(1,1 \%)$ & $1(1,8 \%)$ & \\
1 & $56(61,5 \%)$ & $32(58,2 \%)$ & \\
2 & $13(14,3 \%)$ & $10(18,2 \%)$ & \\
3 & $9(9,9 \%)$ & $5(9,1 \%)$ & \\
4 & $12(13,2 \%)$ & $7(12,7 \%)$ & \\
\hline WHO-Klassifikation & & & \\
MDS-SLD & $3(3,3 \%)$ & $0(0,0 \%)$ & \\
MDS-MLD & $39(42,9 \%)$ & $13(23,6 \%)$ & \\
MDS-EB-1 & $20(22,0 \%)$ & $19(34,5 \%)$ & \\
MDS-EB-2 & $29(31,9 \%)$ & $23(41,8 \%)$ & \\
\hline \hline
\end{tabular}


Tabelle 15: T-Test zum Vergleich metrischer Variablen

\begin{tabular}{lccr}
\hline & Gesamtkollektiv & \multicolumn{1}{c}{ Score-Kollektiv } & \\
\hline Alter & $\mathrm{M}( \pm \mathrm{SD})$ & $\mathrm{M}( \pm \mathrm{SD})$ & $\mathrm{p}$-Wert \\
Hämoglobin & $67,6( \pm 10,70)$ & $66,58( \pm 11,41)$ & 0,59 \\
Hämatokrit & $9,65( \pm 1,69)$ & $9,99( \pm 1,64)$ & 0,23 \\
Erythrozyten & $28,95( \pm 5,18)$ & $29,59( \pm 5,32)$ & 0,48 \\
MCV & $92,19( \pm 0,65)$ & $3,23( \pm 0,66)$ & 0,73 \\
Plättchen & $118,67( \pm 122,07)$ & $105,94( \pm 101,00)$ & 0,39 \\
Leukozyten & $4,32( \pm 4,23)$ & $4,08( \pm 3,84)$ & 0,52 \\
\hline \hline
\end{tabular}

Legende: M: Mittelwert, SD: Standard-Deviation, ${ }^{* * *} \mathrm{p}<0,01 ;{ }^{* *} \mathrm{p}<0,05 ;{ }^{*} \mathrm{p}<0,1$; Erythrozyten $=$ Erythrozytenzahl $* 10^{6}$, Plättchen $=$ Plättchenzahl $* 10^{3}$, Leukozyten $=$ Leukozytenzahl $* 10^{3}$

In Bezug auf die in der Routinediagnostik erhoben morphologischen Parameter (alterskorrigierter Hämatopoieseanteil, Markraumsiderose, Fibrose, CD34+-und CD117+-Blasten) waren die beiden Kollektive bezüglich der Histogramme vergleichbar zusammengesetzt (Abbildung 27 und Abbildung 28). 
A

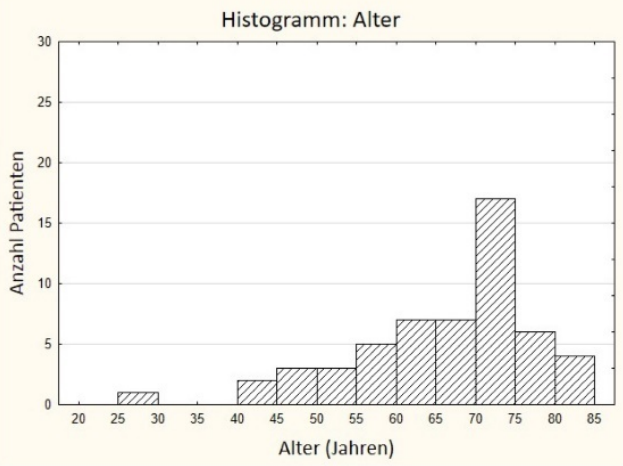

C

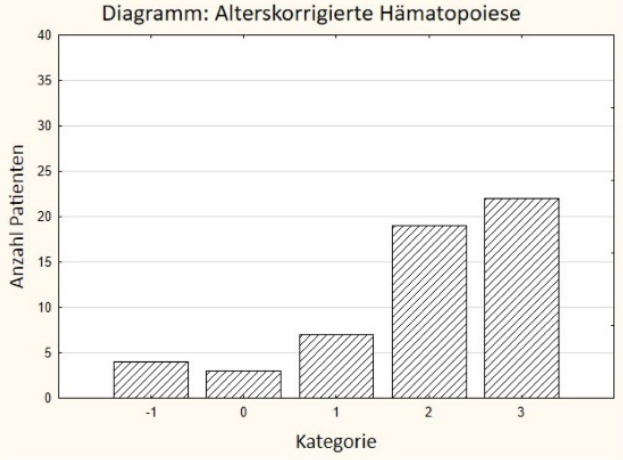

$\mathrm{E}$

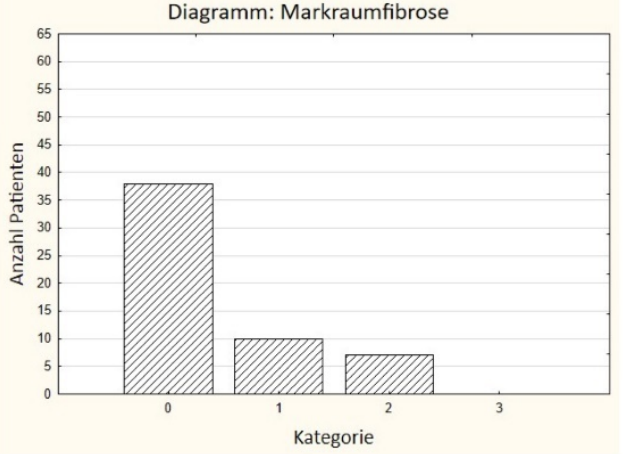

B

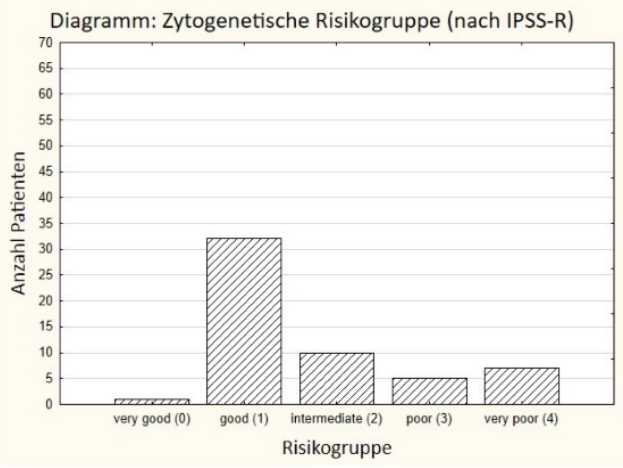

D
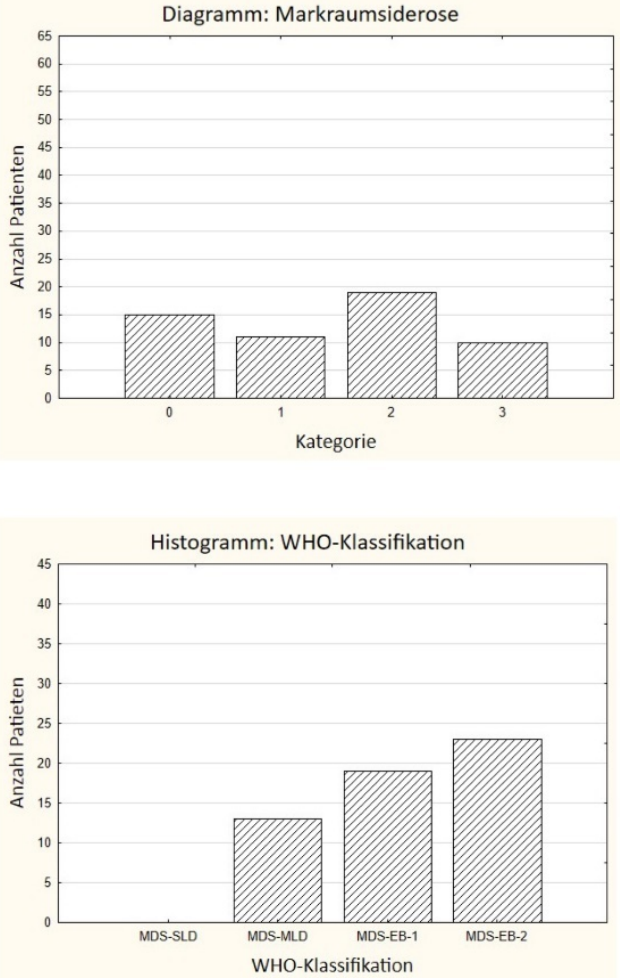

Abbildung 27: Altersverteilung, Zytogenetik und Routinediagnostik im Score-Kollektiv A: Altersverteilung, B: Verteilung der IPSS-R-Risikogruppen, C: Verteilung der alterskorrigierten Hämatopoiese (-1, 0, 1, 2, 3), D: Verteilung der Markraumsiderose (0-3), E: Verteilung der Markraumfibrose (0-3), E: Verteilung der WHO-Subtypen

A

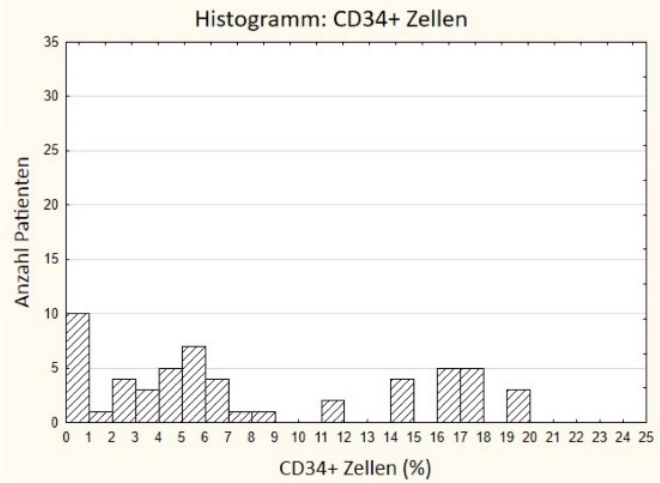

B

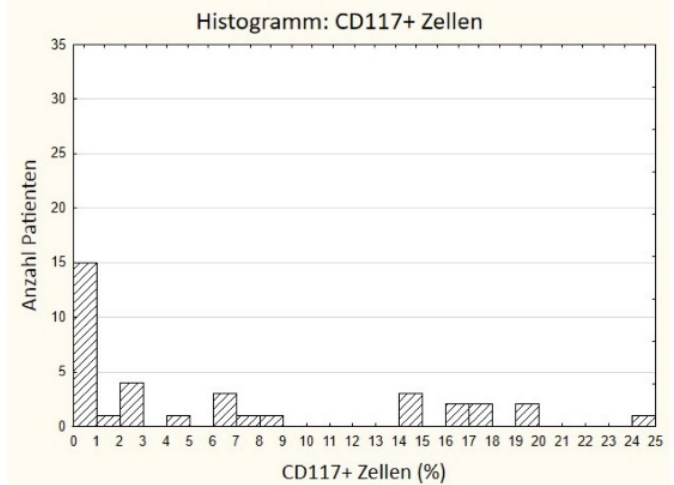

Abbildung 28: Immunhistochemie im Score-Kollektiv A: CD34-Expression von Zellen, B: CD117-Expression von Zellen 
Die bei den Reifungs- und Architekturstörungen beobachteten Schweregrade zeigten unterschiedliche Verteilungsmuster (Abbildung 29 bis Abbildung 31). In den meisten Fällen konnten die betrachteten Parameter gut in verschiedene Kategorien stratifiziert werden. Lediglich bei der Größenvariabilität der Granulopoiese ergab sich ein einseitiges Bild mit 46mal $(84 \%)$ als leicht gestört eingeordneten morphologischen Veränderungen. Schwere Atypien (Grad 3) wurden bei allen Kriterien selten festgestellt. Bei manchen Kriterien (Linksverschiebung der Granulopoiese, ALIP der Granulopoiese, unscharfe Nester der Erythropoiese, Hypolobulierung der Megakaryopoiese und Mikrozytose der Megakaryopoiese) lagen die diagnostizierten Atypien vermehrt im leicht bis mäßig pathologischen Bereich. Im Gegensatz hierzu zeigten sich bei Betrachtung der Makrozytose der Erythropoiese und der Clusterbildung der Megakaryopoiese eine etwa gleiche Aufteilung auf die Gruppen „normal“, „leicht“ und „mäßig““ atypisch. Beim Kriterium Kerngrößenvariabilität der Erythropoiese wurden am häufigsten leichte Veränderungen beobachtet. Die CD34-Expression von Megakaryozyten zeigte ein mit dem Schweregrad (nicht, vereinzelt, teilweise, vollständig) abnehmendes Auftreten.

Hinsichtlich der quantitativen Veränderungen konnte bei allen drei Zellreihen ein ähnliches Verteilungsmuster beobachtet werden. Ein normaler Zellgehalt war am seltensten. In der Regel war der Zellgehalt gesteigert mit einem Maximum bei mäßigen Zunahmen (Kategorie 2). Im Vergleich hierzu kamen Verminderungen in der Zellzahl einer Reihe selten vor. 
A

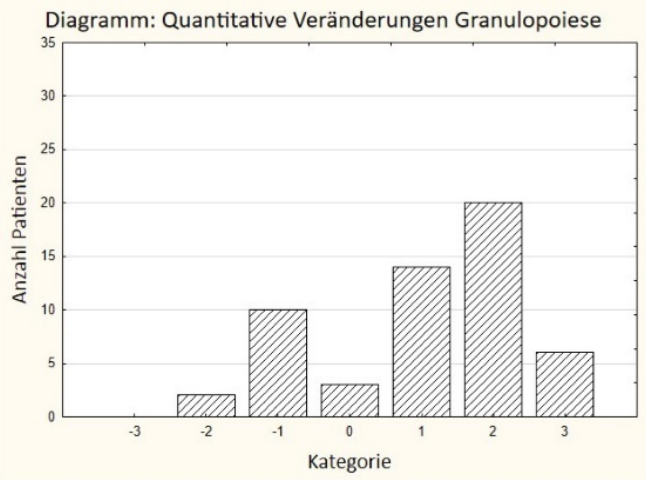

$\mathrm{C}$

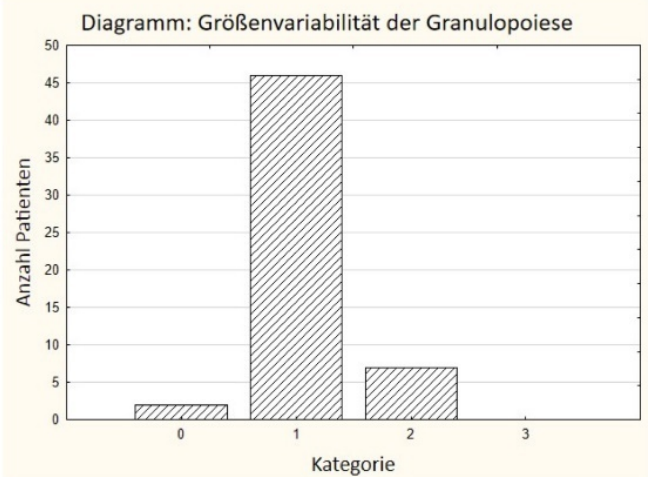

B

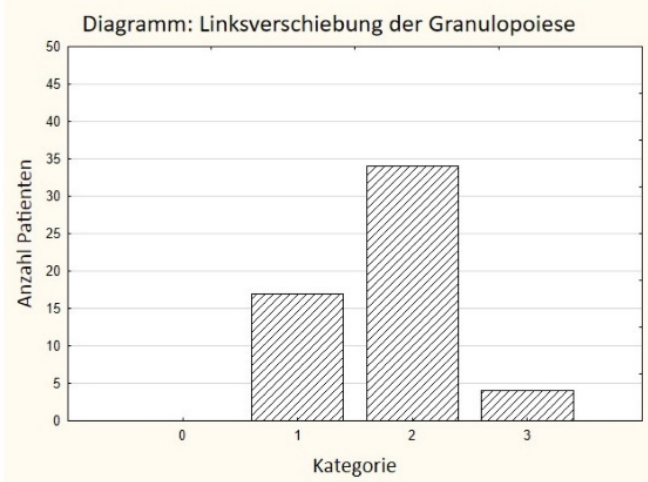

D

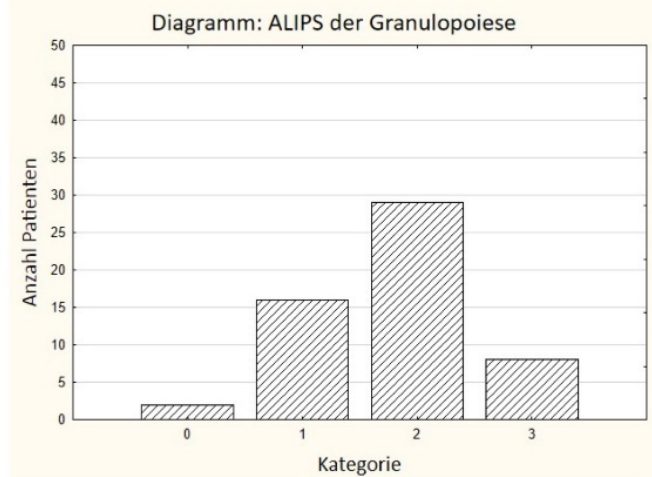

Abbildung 29: Veränderungen der Granulopoiese

A: quantitative Veränderungen (-3 bis 3), B: Linksverschiebung (0-3), C: Größenvariabilität (0-3), D: ALIP (0-3)

A

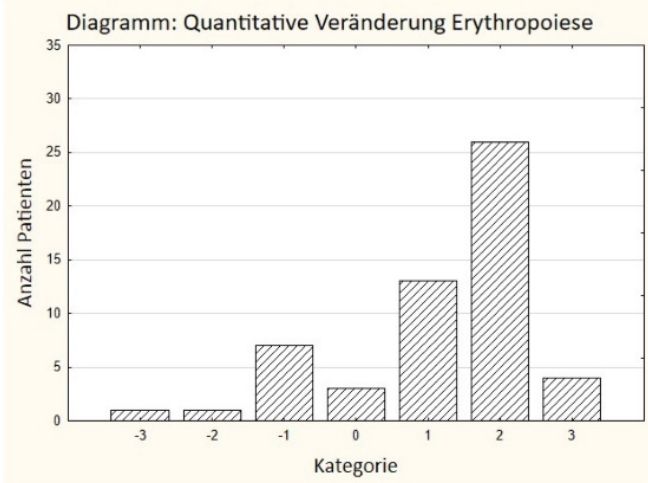

C

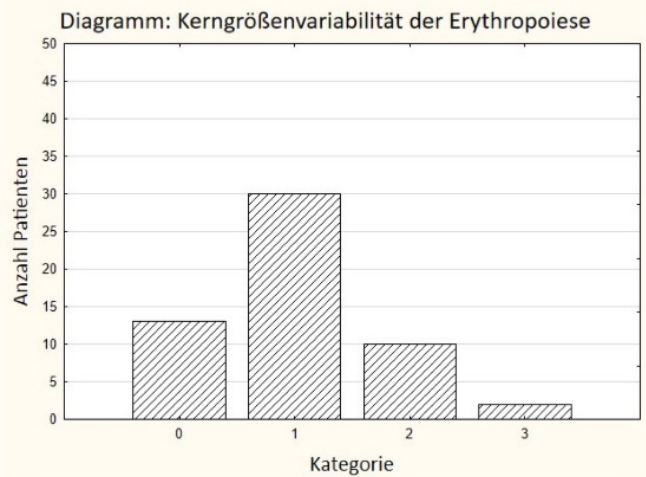

B

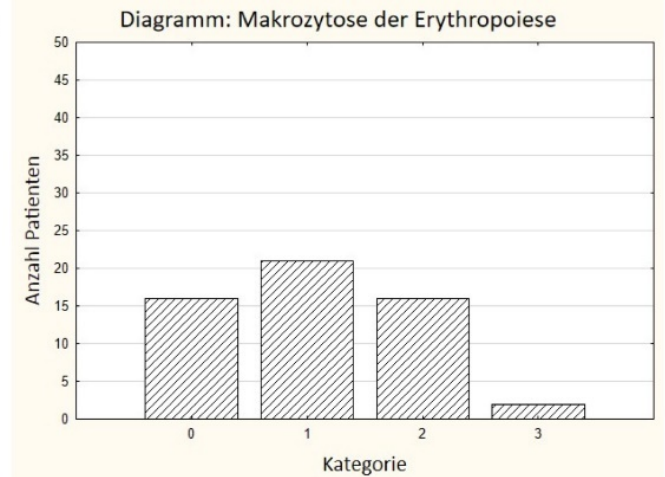

D

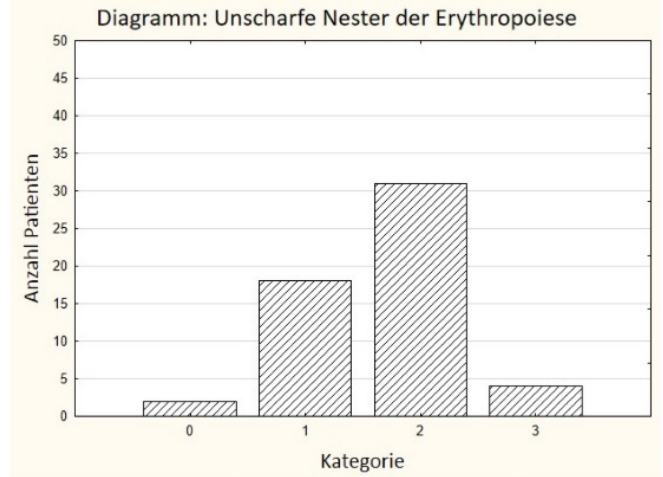

Abbildung 30: Veränderungen der Erythropoiese

A: quantitative Veränderungen (-3 bis 3), B: Makrozytose (0-3), C: Kerngrößenvariabilität (0-3), D: unscharfe Nester (0-3) 
A

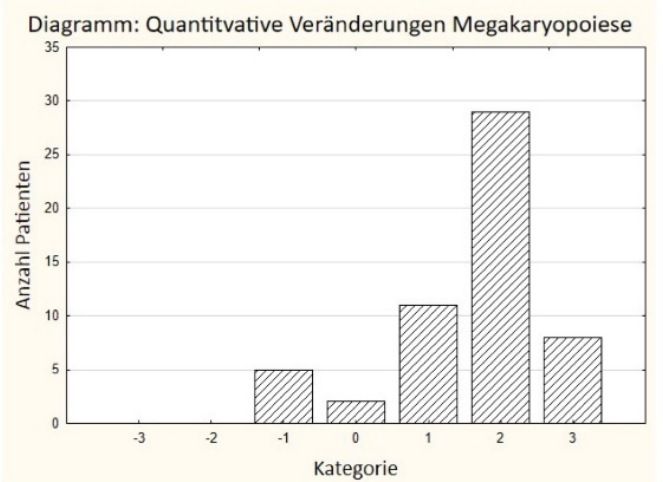

C

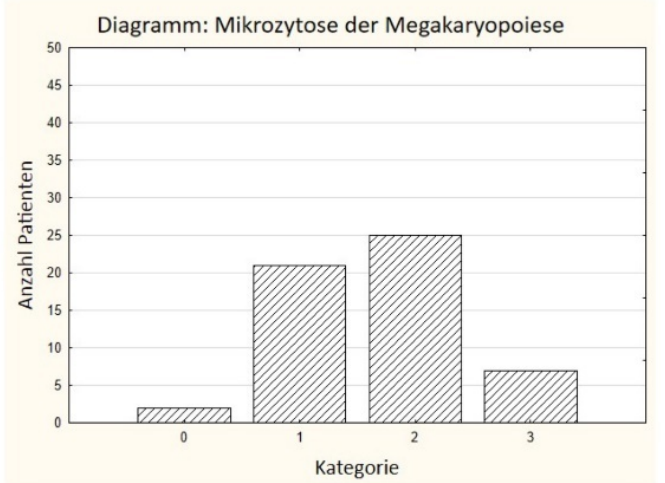

$E$

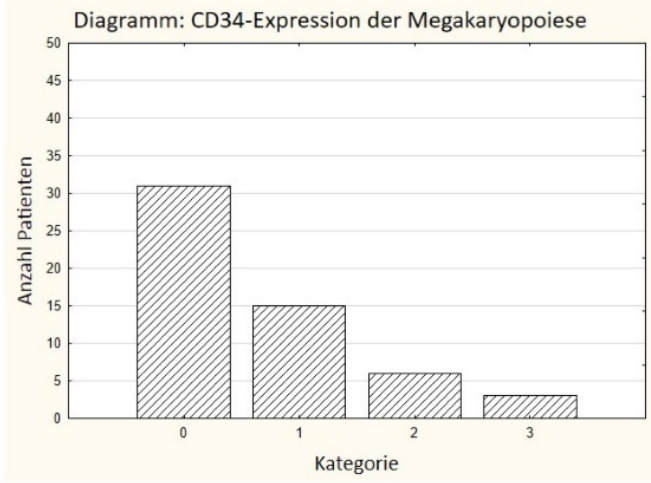

B

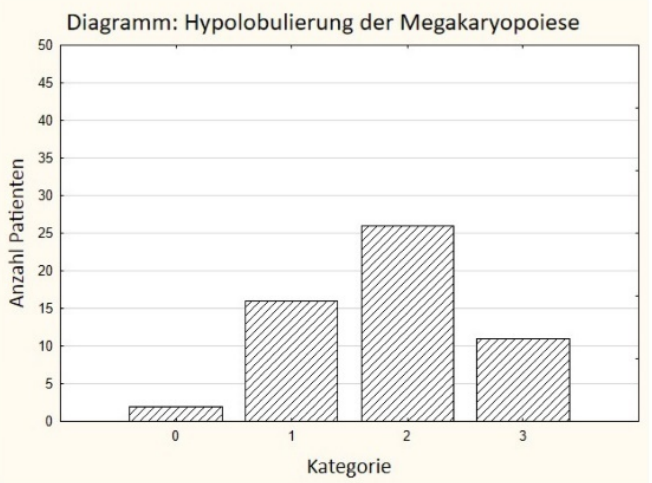

D

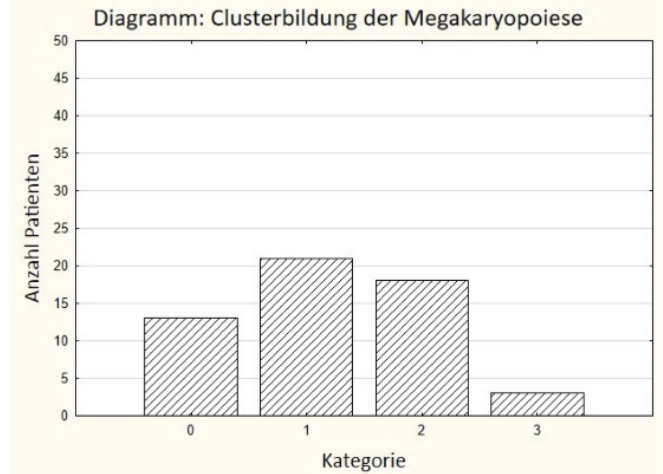

Abbildung 31: Veränderungen der Megakaryopoiese

A: quantitative Veränderungen (-3 bis 3), B: Makrozytose (0-3), C: Kerngrößenvariabilität (0-3), D: unscharfe Nester (0-3)

\subsubsection{Spearman-Rangkorrelation des Score-Kollektivs}

Zuerst wurden die Routineparameter in diesem Score-Kollektiv ausgewertet. Im Vergleich zum Gesamtkollektiv zeigte sich eine weiterhin bestehende Korrelation zwischen der Markraumfibrose mit einem erhöhten IPSS-R $(0,28)$ (Tabelle 16). Der Zusammenhang zwischen der Markraumsiderose und Veränderungen der Erythropoiese war deutlich ausgeprägter (Hämoglobin: -0,43; Hämatokrit: -0,42; Erythrozyten: -0,35), während er für die Fibrose nur teilweise nachweisbar war (Erythrozyten: -0,27). Das Vorhandensein von Lymphozyten im Knochenmark ging ebenfalls mit verminderten Leukozytenzahlen einher $(-0,43)$. 
Tabelle 16: Spearman-Rangkorrelation zwischen Routineparametern und Endpunkten

\begin{tabular}{l|rrrrrrrrr}
\hline $\mathrm{N}=55$ & Hb & HKT & Ery. & MCV & Plt. & Leuk. & KG & Kom. IPSSR \\
\hline Alterskorr. Häm. &,- 19 &, 01 &, 10 &,- 26 &,- 16 &, 27 &, 03 &, 23 &, 07 \\
Lymphozyten &,- 21 &,- 15 &,- 15 &,- 08 &,- 16 & $*_{-}, 43$ &, 03 & $*, 32$ &, 22 \\
Siderose & $*_{-}, 43$ & $*_{-}, 42$ & $*_{-}, 35$ &, 01 &,- 02 &,- 10 &, 21 &, 16 &, 23 \\
Fibrose &,- 18 &,- 24 & $*_{-}, 27$ &, 07 &, 13 &,- 18 &, 11 &, 26 & $* 0,28$ \\
CD34 -Zellen &,- 09 &,- 16 &,- 09 &, 13 &,- 06 &,- 15 &,- 23 &, 03 &, 10 \\
CD117+-Zellen &,- 08 &,- 02 &,- 08 &,- 05 &, 20 &, 13 &,- 30 &, 11 &, 02 \\
\hline \hline
\end{tabular}

Legende: Hb: Hämoglobin HKT: Hämatokrit, Ery.: Erythrozyten, MCV: Mean corpuscular volume, Plt.: Plättchen, Leu.: Leukozyten, KG: Klongröße, Kom.: Komplex, Alterkorr. Häm: Alterskorrigierte Hämatopoiese, ${ }^{*} \mathrm{p}<$ 0,05

Die Analyse der Spearman-Rangkorrelation zwischen Morphologie und IPSS-R zeigte vor allem bei Veränderungen der Megakaryopoiese einen Zusammenhang mit einem erhöhten zytogenetischen Risiko. Am deutlichsten und signifikant war eine positive Korrelation bei der Vermehrung von Megakaryozyten im Knochenmark (0,39). Ebenfalls signifikant mit IPSS-R korrelierte eine Verminderung der Granulopoiese (-0,27).

Auch bei den sekundären Endpunkten (Klongröße, komplexer Karyotyp und Laborparameter) ergab sich ein Zusammenhang mit morphologischen Veränderungen im Knochenmark (Tabelle 17). Bei komplexen Karotypen fanden sich vermehrt unscharfe Nester der Erythropoiese $(0,28)$, CD $34^{+}$-Megakaryozyten $(0,28)$ sowie eine Vermehrung der Megakaryopoiese $(0,29)$. Letzteres korrelierte zusätzlich signifikant mit der erhöhten Klongröße $(0,38)$. Eine quantitative Vermehrung der Erythropoiese im Knochenmark korrelierte signifikant positiv mit Parametern der roten Reihe im peripheren Blut (Hämoglobin: 0,39; Hämatokrit: 0,53, Erythrozyten: 0,45) und negativ mit Plättchen im peripheren Blut $(-0,36)$. In weniger ausgeprägter Form bestand dieser Zusammenhang ebenfalls für die Entstehung von unscharfen Nestern (Erythrozyten: 0,27, Plättchen: -0,30). Dysplastische Veränderungen an der Megakaryozytenmorphologie im Knochenmark gingen mit Veränderungen der peripheren roten Blutkörperchen einher. Eine Vermehrung von Megakaryozyten und eine Clusterbildung korrelierten mit einem erniedrigten Hämatokrit (-0,27 bzw. -0,32). Hypolobulierung stand in einem Zusammenhang mit verminderten Hämoglobin $(-0,28)$ und Erythrozytenzahlen (0,28). Letzteres galt auch für eine Mikrozytose (Erythrozytenzahl: -0,27). 
Tabelle 17: Spearman-Rangkorrelation zwischen Morphologiescore und Endpunkten

\begin{tabular}{l|rrrrrrrrr}
\hline N=55 & Hb & HKT & Ery. & MCV & Plt. & Leuk. & KG & Kom. IPSSR \\
\hline GP_QUA &, 03 &,- 01 &, 11 &,- 10 & $*, 32$ & $* 0,32$ &,- 25 &,- 14 & $*_{-}, 27$ \\
GP_LV &,- 18 &,- 21 &,- 11 &, 02 &, 12 &,- 10 &, 05 &,- 02 &, 01 \\
GP_GV &, 02 &, 01 &,- 02 &, 18 &, 08 &, 07 &,- 06 &,- 08 &,- 06 \\
GP_ALIP &,- 16 &,- 18 &,- 13 &, 11 &, 24 &, 05 &,- 07 &,- 13 &,- 15 \\
EP_QUA & $*, 39$ & $*, 53$ & $*, 45$ &,- 15 & $*-, 36$ &,- 08 &, 20 &, 15 &, 05 \\
EP_MAZ &,- 16 &,- 19 &,- 21 &, 13 &,- 11 &,- 22 &, 17 &,- 09 &, 06 \\
EP_KGV &,- 11 &,- 14 &,- 10 &, 03 &,- 06 &,- 15 &, 04 &, 01 &, 13 \\
EP_UN &, 24 &, 24 & $*, 27$ &,- 10 & $*-, 30$ &,- 17 &, 09 & $*, 28$ &, 10 \\
MP_QUA &,- 22 & $*-, 27$ &,- 24 &, 08 &, 05 &,- 14 & $*, 38$ & $*, 29$ & $*, 39$ \\
MP_HL & $*-, 28$ &,- 24 & $*_{-}, 28$ &, 14 &,- 12 &,- 16 &, 10 &, 10 &, 21 \\
MP_MIZ &,- 26 &,- 18 & $*-, 27$ &, 21 &,- 07 &,- 03 &, 05 &,- 08 &, 14 \\
MP_CL &,- 25 & $*-, 32$ &,- 24 &, 02 &,- 06 &,- 11 &, 01 &, 02 &, 22 \\
MP_CD34 &,- 03 &,- 07 &,- 03 &,- 16 &,- 16 &, 07 &,- 17 & $*, 28$ &, 13 \\
\hline \hline
\end{tabular}

Legende: Hb: Hämoglobin HKT: Hämatokrit, Ery.: Erythrozyten, MCV: mean corpuscular volume, Plt.: Plättchen, Leu.: Leukozyten, KG: Klongröße, Kom.: Komplex, GP_QUA: quantitative Veränderungen der Granulupoiese, GP_LV: Linksverschiebung, GP_GV: Größenvariabilität, GP_ALIP: abnormal localization of immature precursors, EP_QUA: quantitative Veränderungen der Erythropoiese, EP_MAZ: Makrozytose, EP_KGV: Kerngrößenvariabilität, EP_UN: unscharfe Nester, MP_QUA: quantitative Veränderungen der Megakaryopoiese, MP_HL: Hypolobulierung, MP_MIZ: Mikrozytose, MP_CL: Clusterbildung, MP_CD34: CD34-Expression, * $\mathrm{p}<0,05$

\subsubsection{WHO-Klassifikation und Zytogenetik}

Analog zum Gesamtkollektiv wurde untersucht, ob die abschließende Diagnose nach der WHO-Klassifikation mit dem zytogenetischen Risiko in Verbindung steht. Die Analyse der genetischen IPSS-R-Risikogruppen in den Kategorien MDS-MLD, MDS-EB-1 und MDSEB-2 im Score-Kollektiv ergab eine unterschiedliche Verteilung. Es ließ sich erkennen, dass mehr Patienten mit MDS-EB-1 oder MDS-EB-2 (63 \% bzw. 65 \%) ein Risiko der Kategorie „good" (1 Punkt) haben als die Patienten mit einer MDS-MLD (38\%). Wie in Abbildung 32 dargestellt, nahm in gleichem Maß auch der Anteil von Patienten mit erhöhtem zytogenetischen Risiko (IPSS-R-Punktzahl 2-4) bei MDS-MLD zu. 
A

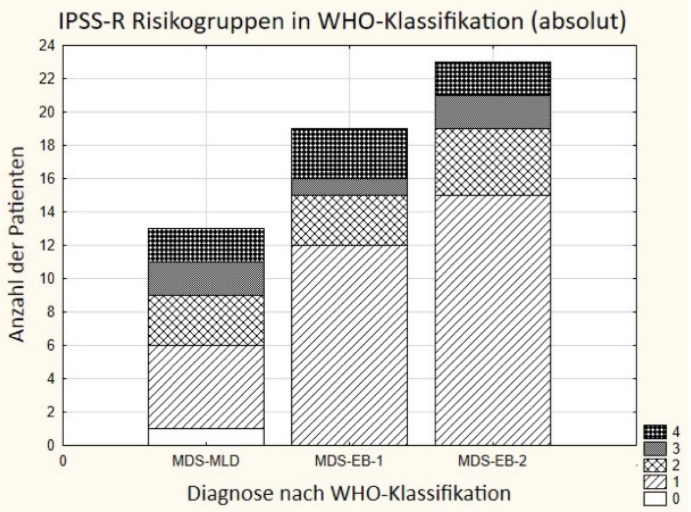

B

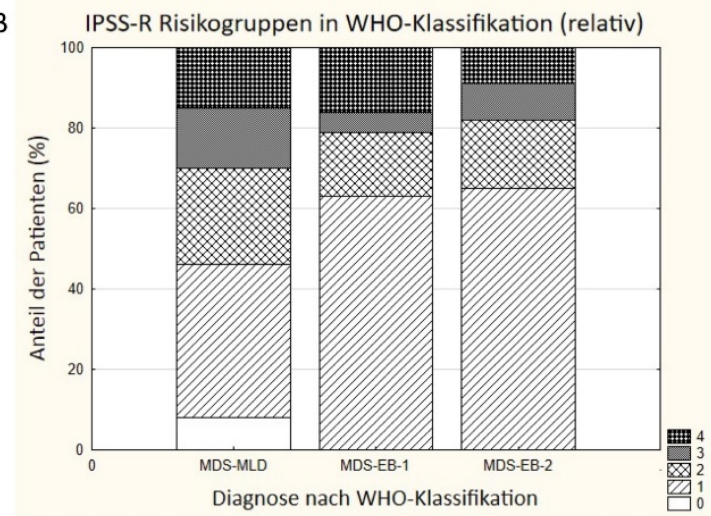

Abbildung 32: IPSS-R-Risikogruppen in der WHO-Klassifikation des Score-Kollektivs A: Darstellung der absoluten Häufigkeiten von zytogenetischen Risikogruppen je WHO-Kategorie; B: Darstellung der relativen Häufigkeiten von zytogenetischen Risikogruppen je WHO-Kategorie

Zur Ermittlung eines Zusammenhangs wurde eine Spearman-Rangkorrelation durchgeführt und die Kategorien mit aufsteigenden Nummern (MDS-MLD $=2$, MDS-EB-1 $=3$ und MDS-EB-2 = 4) kodiert. Eine signifikante Korrelation zeigte sich nicht (Rangkorrelationskoeffizient: $-0,11, \mathrm{p}$-Wert $>0,05$.

Um einem möglichen Zusammenhang zwischen diesen beiden Variablen weiter nachzugehen, wurde eine Regressionsanalyse durchgeführt. Es konnte kein statistisch signifikanter Zusammenhang festgestellt werden (Tabelle 18).

Tabelle 18: Regression des IPSS-R mit dem Prädiktor WHO-Subtyp im Score-Kollektiv

\begin{tabular}{lrc}
\hline $\mathrm{N}=55$ & $\mathrm{p}$-Wert & Odds Ratio 95\% -KI \\
\hline WHO-Subtyp & & \\
MDS-MLD (Ref.) & & - \\
MDS-EB-1 & 0,55 & $0,66[0,17 ; 2,58]$ \\
MDS-EB-2 & 0,4 & $0,57[0,15 ; 2,10]$ \\
\hline \hline
\end{tabular}

Legende: Ref.: Referenzkategorie, ${ }^{* * *} \mathrm{p}<0,01$; $^{* *} \mathrm{p}<0,05$; $^{*} \mathrm{p}<0,1$

\subsubsection{Logistische Regression im Score-Kollektiv}

Um den Zusammenhang zwischen den morphologischen Kriterien und dem IPSS-R genauer beschreiben zu können, wurden auch für diese Einflussvariablen ordinale logistische Regressionen durchgeführt. Ein Modell mit den bereits erhobenen morphologischen Daten der Routinediagnostik (alterskorrigierte Hämatopoiese, Siderose, Fibrose, CD34+-Zellen) sowie den möglichen Störvariablen (Alter, Geschlecht, Ätiologie) wurde gebildet. Zusätzlich wurde die histomorphologische Beurteilung der Reifungs- und Architekturstörung einer Zellreihe (Granulopoiese, Erythropoiese und Megakaryopoiese) hinzugefügt. 


\subsubsection{Veränderungen der Granulopoiese}

In einem ersten Untersuchungsschritt wurde eine univariate Analyse der verschiedenen Parameter der Granulopoiese durchgeführt (Tabelle 19). Hierbei ergab sich, dass eine Vermehrung der Granulopoiese mit einem niedrigeren Risiko für einen hohen IPSS-R einherging.

Tabelle 19: Univariate Regression des IPPS-R mit Prädiktoren der Granulopoiese

\begin{tabular}{lrr}
\hline $\mathrm{N}=55$ & $\mathrm{p}$-Wert & Odds Ratio 95\%-KI \\
\hline $\begin{array}{l}\text { Quantitative Veränderung GP } \\
\quad / 1 \text { (Ref.) }\end{array}$ & \multicolumn{2}{c}{-} \\
$\quad+2 /+3$ & 0,02 & $* * 0,22[0,06 ; 0,79]$ \\
$\quad-1 /-2 /-3$ & 0,89 & $0,91[0,23 ; 3,52]$ \\
\hline Linksverschiebung & 0,94 & $1,03[0,43 ; 2,46]$ \\
\hline Größenvariabilität & 0,70 & $0,78[0,22 ; 2,79]$ \\
\hline ALIP & 0,31 & $0,70[0,35 ; 1,38]$ \\
\hline \hline
\end{tabular}

Legende: Ref.: Referenzkategorie, ${ }^{* * *} \mathrm{p}<0,01 ;{ }^{* *} \mathrm{p}<0,05 ;{ }^{*} \mathrm{p}<0,1$; GP: Granulopoiese

Im nächsten Schritt wurde ein größeres Modell unter Einschluss der Routineparameter und möglicher Störvariablen gebildet. Auch hier zeigte sich, dass eine Vermehrung der Granulopoiese mit einem verminderten Risiko für einen hohen IPSS-R einherging (OR: 0,09; 95\%-KI [0,02; 0,51]) (Tabelle 20). Des Weiteren korrelierte in diesem Modell der Routineparameter „alterskorrigierte Hämatopoiese“ mit einem erhöhten IPSS-R. Das breite Konfidenzintervall zeigte, dass die Stärke dieses Einflusses nur schwer mit Sicherheit eingeschätzt werden konnte. Auch die Fibrose wies einen deutlichen Einfluss in diesem Modell (OR: 3,17, 95\%-KI: 1,09-9,21) auf. Für andere Reifungs- oder Architekturstörungen der Granulopoiese zeigte sich kein relevanter Zusammenhang mit dem IPSS-R. 
Tabelle 20: Ordinale logistische Regression des IPSS-R mit Variablen der Granulopoiese

\begin{tabular}{|c|c|c|}
\hline $\mathrm{N}=55$ & $\mathrm{p}$-Wert & Odds Ratio $95 \%-\mathrm{KI}$ \\
\hline \multicolumn{3}{|c|}{ Alterskorrigierte Hämatopoiese } \\
\hline 0/1 (Ref.) & & - \\
\hline-1 & 0,68 & $2,06[0,07 ; 65,22]$ \\
\hline+2 & 0,01 & $* * 33,59[2,10 ; 538,37]$ \\
\hline+3 & 0,01 & $* * 30,73[2,20 ; 428,74]$ \\
\hline \multicolumn{3}{|l|}{ Lymphozyten } \\
\hline nein (Ref.) & & - \\
\hline ja & 0,38 & $2,97[0,26 ; 34,04]$ \\
\hline Eisen & 0,57 & $1,27[0,56 ; 2,90]$ \\
\hline Fibrose & 0,03 & $* * 3,17[1,09 ; 9,21]$ \\
\hline CD34+-Zellen & 0,83 & $0,99[0,88 ; 1,10]$ \\
\hline \multicolumn{3}{|c|}{ Quantitative Veränderung GP } \\
\hline 0/1 (Ref.) & & - \\
\hline$+2 /+3$ & $<0,01$ & $* * * 0,09[0,02 ; 0,51]$ \\
\hline$-1 /-2 /-3$ & 0,40 & $0,43[0,06,3,10]$ \\
\hline Linksverschiebung & 0,42 & $2,29[0,31 ; 17,11]$ \\
\hline Größenvariabilität & 0,47 & $2,09[0,29 ; 15,20]$ \\
\hline ALIP & 0,88 & $0,86[0,13 ; 5,90]$ \\
\hline \multicolumn{3}{|l|}{ Ätiologie } \\
\hline Primär (Ref.) & & - \\
\hline Sekundär & 0,06 & $* 7,47[0,93 ; 59,71]$ \\
\hline Alter & 0,01 & $* * 0,92[0,86 ; 0,98]$ \\
\hline \multicolumn{3}{|l|}{ Geschlecht } \\
\hline Männlich (Ref.) & & - \\
\hline Weiblich & 0,16 & $0,36[0,09 ; 1,49]$ \\
\hline
\end{tabular}

Legende: Ref.: Referenzkategorie, ${ }^{* * *} \mathrm{p}<0,01 ;{ }^{* *} \mathrm{p}<0,05{ }^{*} \mathrm{p}<0,1$; GP: Granulopoiese

\subsubsection{Veränderungen der Erythropoiese}

Die Betrachtung der Parameter der Erythropoiese in der univariaten Regressionsanalyse ergab keinen signifikanten Einfluss auf das genetische Risiko laut IPSS-R (Tabelle 21). 
Tabelle 21: Univariate Regression des IPPS-R mit Prädiktoren der Erythropoiese

\begin{tabular}{|c|c|c|}
\hline $\mathrm{N}=55$ & p-Wert & Odds Ratio $95 \%-\mathrm{KI}$ \\
\hline \multicolumn{3}{|c|}{ Quantitative Veränderung EP } \\
\hline 0/1 (Ref.) & & - \\
\hline$+2 /+3$ & 0,56 & $0,71[0,22 ; 2,29]$ \\
\hline$-1 /-2 /-3$ & 0,42 & $0,52[0,11 ; 2,55]$ \\
\hline Makrozytose & 0,69 & $1,13[0,62 ; 2,04]$ \\
\hline Kerngrößenvariabilität & 0,38 & $1,36[0,68 ; 2,69]$ \\
\hline Unscharfe Nester & 0,31 & $1,52[0,68 ; 3,38]$ \\
\hline
\end{tabular}

Dieser Befund bestätigte sich unter Einschluss der Routine- und möglicher Störvariablen (Tabelle 22).

Tabelle 22: Ordinale logistische Regression des IPSS-R mit Variablen der Erythropoiese

\begin{tabular}{|c|c|c|}
\hline $\mathrm{N}=55$ & p-Wert & Odds Ratio $95 \%-\mathrm{KI}$ \\
\hline \multicolumn{3}{|c|}{ Alterskorrigierte Hämatopoiese } \\
\hline 0/1 (Ref.) & & - \\
\hline-1 & 0,45 & $3,85[0,12 ; 125,28]$ \\
\hline+2 & 0,08 & $* 7,75[0,77 ; 77,94]$ \\
\hline+3 & 0,05 & $* 8,84[1,00 ; 78,35]$ \\
\hline \multicolumn{3}{|l|}{ Lymphozyten } \\
\hline nein (Ref.) & & - \\
\hline ja & 0,48 & $2,21[0,25 ; 19,92]$ \\
\hline Eisen & 0,34 & $1,48[0,67 ; 3,29]$ \\
\hline Fibrose & 0,06 & $* 2,59[0,95 ; 7,09]$ \\
\hline CD34+-Zellen & 0,42 & $0,95[0,85 ; 1,07]$ \\
\hline \multicolumn{3}{|c|}{ Quantitative Veränderung EP } \\
\hline 0/1 (Ref.) & & - \\
\hline$+2 /+3$ & 0,73 & $0,76[0,15 ; 3,73]$ \\
\hline$-1 /-2 /-3$ & 0,37 & $0,35[0,03 ; 3,48]$ \\
\hline Makrozytose & 0,15 & $0,40[0,12 ; 1,37]$ \\
\hline Kerngrößenvariabilität & 0,19 & $2,86[0,60 ; 13,70]$ \\
\hline Unscharfe Nester & 0,43 & $1,82[0,42 ; 7,88]$ \\
\hline \multicolumn{3}{|l|}{ Ätiologie } \\
\hline Primär (Ref.) & & - \\
\hline Sekundär & 0,13 & $4,93[0,63 ; 38,82]$ \\
\hline Alter & 0,02 & $* * 0,93[0,87 ; 0,99]$ \\
\hline \multicolumn{3}{|l|}{ Geschlecht } \\
\hline Männlich (Ref.) & & - \\
\hline Weiblich & 0,23 & $0,39[0,09 ; 1,79]$ \\
\hline
\end{tabular}

Legende: Ref.: Referenzkategorie, ${ }^{* * *} \mathrm{p}<0,01$; $^{* *} \mathrm{p}<0,05$; $^{*} \mathrm{p}<0,1$, EP: Erythropoiese 


\subsubsection{Veränderungen der Megakaryopoiese}

In der univariaten ordinalen Regression zeigte sich ein deutlicher Einfluss einer Vermehrung der Megakaryopoiese als Prädiktor für einen ungünstigen IPSS-R (Tabelle 23). Ein Trend in diese Richtung war auch bei der Clusterbildung der Megakaryopoese zu erkennen.

Tabelle 23: Univariate Regression des IPPS-R mit Prädiktoren der Megakaryopoiese

\begin{tabular}{lcc}
\hline $\mathrm{N}=55$ & $\mathrm{p}$-Wert & Odds Ratio 95\%-KI \\
\hline Quantitative Veränderung MP & \multicolumn{2}{c}{-} \\
$\quad 0 / 1$ (Ref.) & 0,90 & $1,16[0,12 ; 11,62]$ \\
$-1 /-2 /-3$ & 0,04 & $* * 4,63[1,09 ; 19,71]$ \\
$\quad+2 /+3$ & 0,17 & $1,60[0,82 ; 3,07]$ \\
Hypolobulierung & 0,27 & $1,48[0,74 ; 2,95]$ \\
\hline Mikrozytose & 0,07 & $* 1,77[0,96 ; 3,27]$ \\
\hline Clusterbildung & 0,21 & $1,48[0,80 ; 2,73]$ \\
\hline CD34-Expression & & \\
\hline \hline
\end{tabular}

Legende: Ref.: Referenzkategorie, ${ }^{* * *} \mathrm{p}<0,01 ;{ }^{* *} \mathrm{p}<0,05 ;{ }^{*} \mathrm{p}<0,1 ;$ MP: Erythropoiese

Betrachtete man den Einfluss von morphologischen Kriterien der Megakaryopoiese auf den IPSS-R unter Einschluss der Routineparameter und möglicher Störvariablen, ergab sich ein Zusammenhang mit der Vermehrung der Megakaryozyten im Knochenmark (Tabelle 24). Die hohe OR und das überwiegend über 1 liegende Konfidenzintervall zeigten den potentiell starken Einfluss dieser Variable. Unter der Anwendung einer größeren Stichprobe könnte dieser Parameter an Signifikanz zunehmen. Gleiches gilt in diesem Modell für die Lymphozyten. Der Einfluss der Clusterbildung war in diesem Modell nicht mehr nachzuweisen. Wahrscheinlich wird das genetische Risiko durch andere Variablen besser vorausgesagt. 
Tabelle 24: Ordinale logistische Regression des IPSS-R mit Variablen der Megakaryopoiese

\begin{tabular}{|c|c|c|}
\hline $\mathrm{N}=55$ & p-Wert & Odds Ratio $\quad 95 \%-\mathrm{KI}$ \\
\hline \multicolumn{3}{|c|}{ Alterskorrigierte Hämatopoiese } \\
\hline 0/1 (Ref.) & & - \\
\hline-1 & 0,74 & $0,56[0,02 ; 18,02]$ \\
\hline+2 & 0,25 & $4,24[0,36 ; 50,64]$ \\
\hline+3 & 0,20 & $4,32[0,45 ; 41,45]$ \\
\hline \multicolumn{3}{|l|}{ Lymphozyten } \\
\hline nein (Ref.) & & - \\
\hline ja & 0,07 & $* 7,36[0,87 ; 62,54]$ \\
\hline Eisen & 0,71 & $0,85[0,35 ; 2,04]$ \\
\hline Fibrose & 0,12 & $2,25[0,82 ; 6,14]$ \\
\hline CD34+-Zellen & 0,88 & $0,99[0,89 ; 1,10]$ \\
\hline \multicolumn{3}{|c|}{ Quantitative Veränderung MP } \\
\hline 0/1 (Ref.) & & - \\
\hline$-1 /-2 /-3$ & 0,77 & $1,55[0,08 ; 29,52]$ \\
\hline$+2 /+3$ & 0,08 & $* 5,66[0,81 ; 39,46]$ \\
\hline Hypolobulierung & 0,88 & $0,91[0,27 ; 3,06]$ \\
\hline Mikrozytose & 0,99 & $1,01[0,29 ; 3,50]$ \\
\hline Clusterbildung & 0,77 & $1,19[0,38 ; 3,74]$ \\
\hline CD34-Expression & 0,61 & $0,77[0,28 ; 2,09]$ \\
\hline \multicolumn{3}{|l|}{ Ätiologie } \\
\hline Primär (Ref.) & & - \\
\hline Sekundär & 0,01 & **20,51 [1,93; 218,06] \\
\hline Alter & 0,10 & $*_{0,95}[0,89 ; 1,01]$ \\
\hline \multicolumn{3}{|l|}{ Geschlecht } \\
\hline Männlich (Ref.) & & - \\
\hline Weiblich & 0,23 & $0,39[0,08 ; 1,83]$ \\
\hline
\end{tabular}

Legende: Ref.: Referenzkategorie, ${ }^{* * *} \mathrm{p}<0,01$; ${ }^{* *} \mathrm{p}<0,05$; $^{*} \mathrm{p}<0,1 ;$ MP: Megakaryopoiese

\subsubsection{Volles logistisches Modell}

Für die endgültige Analyse wurden alle in den vorherigen Tests als relevant erkannten Kriterien in einem ordinalen logistischen Modell zusammengefasst. Dies diente dazu, den gegenseitigen Einfluss der Variablen zu adjustieren. Es zeigte sich ein weiterhin bestehender starker protektiver Effekt der Vermehrung der Granulopoiese ebenso wie ein möglicherweise erhöhtes genetisches Risiko bei gesteigerter Megakaryopoiese. Ein hoher potentieller Einfluss konnte ebenfalls für eine gesteigerte alterskorrigierte Hämatopoiese (Grad 3) gezeigt werden (OR: 10, 75; 95\%-KI: 0,88-131,55). Scheinbar wurde der Einfluss der Fibrose durch andere Kriterien in diesem Modell besser erklärt. Auch wenn der p-Wert keine hohe Signifikanz des Kriteriums suggerierte (p: 0,11), deuteten OR und KI auf eine hohe mögliche 
Relevanz dieses Kriteriums hin (OR: 2,34; 95\%-KI: 0,82-6,69). Ebenfalls deutlich war der Einfluss einer sekundären Ätiologie. Hinsichtlich des Alters bestätigte sich der Trend, der bereits in allen vorherigen Regressionen auftrat. Ältere Patienten schienen ein etwas besseres zytogenetisches Risiko zu haben als jüngere.

Tabelle 25: Volles Modell der ordinalen logistische Regression des IPSS-R

\begin{tabular}{|c|c|c|}
\hline $\mathrm{N}=55$ & p-Wert & Odds Ratio $95 \%-\mathrm{KI}$ \\
\hline \multicolumn{3}{|c|}{ Alterskorrigierte Hämatopoiese } \\
\hline 0/1 (Ref.) & & - \\
\hline-1 & 0,77 & $0,61[0,02 ; 16,14]$ \\
\hline+2 & 0,14 & $7,27[0,52 ; 101,80]$ \\
\hline+3 & 0,06 & $* 10,75[0,88 ; 131,55]$ \\
\hline \multicolumn{3}{|l|}{ Lymphozyten } \\
\hline nein (Ref.) & & - \\
\hline ja & 0,21 & $3,83[0,46 ; 31,77]$ \\
\hline Eisen & 0,87 & $0,93[0,40 ; 2,18]$ \\
\hline Fibrose & 0,11 & $2,34[0,82 ; 6,69]$ \\
\hline CD $34^{+}$-Zellen & 0,85 & $1,01[0,90 ; 1,14]$ \\
\hline \multicolumn{3}{|c|}{ Quantitative Veränderung GP } \\
\hline 0/1 (Ref.) & & - \\
\hline$-1 /-2 /-3$ & 0,73 & $0,72[0,11 ; 4,57]$ \\
\hline$+2 /+3$ & $<0,01$ & $* * * 0,09[0,02 ; 0,47]$ \\
\hline \multicolumn{3}{|c|}{ Quantitative Veränderung MP } \\
\hline 0/1 (Ref.) & & - \\
\hline$-1 /-2 /-3$ & 0,70 & $0,56[0,03 ; 10,97]$ \\
\hline$+2 /+3$ & 0,06 & $* 5,84[0,92 ; 37,20]$ \\
\hline \multicolumn{3}{|l|}{ Ätiologie } \\
\hline Primär & & - \\
\hline Sekundär & $<0,01$ & ***17,65 [2,10; 148,64] \\
\hline Alter & 0,08 & $* 0,94[0,88 ; 1,01]$ \\
\hline \multicolumn{3}{|l|}{ Geschlecht } \\
\hline Männlich (Ref.) & & - \\
\hline Weiblich & 0,16 & $0,34[0,08 ; 1,54]$ \\
\hline
\end{tabular}

Legende: Ref.: Referenzkategorie, ${ }^{* * *} \mathrm{p}<0,01 ;{ }^{* *} \mathrm{p}<0,05 ;{ }^{*} \mathrm{p}<0,1$; GP: Granulopoiese, MP: Megakaryopoiese 


\section{Diskussion}

\subsection{Limitationen der Arbeit}

\subsubsection{Histomorphologische Auswertung}

In der vorliegenden Arbeit wurden histomorphologische Daten in semi-quantitativen Kategorien, wie Reifungs- und Architekturstörungen, erhoben. Eine grundsätzliche Limitation dieser Methodik ist die Gefahr einer Untersucherabhängigkeit der Daten. Diese Problematik ist in Bezug auf die zytologisch-morphologische Diagnostik bei MDS in letzter Zeit vielfach beleuchtet worden. So konnten Naqvi et al. (2011) in einer Studie mit 915 Patienten nachweisen, dass in $12 \%$ der Fälle ein Unterschied zwischen der Einweisungsdiagnose eines peripheren Krankenhauses im Vergleich zur eigenen Diagnose bestand. Diese war an einem universitären Zentrum von drei unterschiedlichen Pathologen gestellt worden (Naqvi et al. 2011). Auch in anderen Studien, von denen im Folgenden die wichtigsten besprochen werden, wurde die Reproduzierbarkeit der Beurteilung von WHO-Klassifikation, Blastenanteil oder Dysplasiekriterien geprüft. Diesbezüglich wurde meist die Interrater-Reliabilität (Urteiler-Übereinstimmung) bestimmt.

In Bezug auf eine Einordnung in die WHO-Klassifikation konnte in einer Untersuchung von 100 Präparaten gezeigt werden, dass die Interrater-Übereinstimmung bei zytologischer Begutachtung von Patienten mit bekanntem MDS bei den Gruppen RCMD (74 \%), RAEB-1 (84\%) und RAEB-2 (90\%) gut ist, jedoch nicht in den Kategorien RA (25\%) und RCUD (40\%) (Font et al. 2013). Dies hatte die Forschungsgruppe zum Anlass genommen, die Übereinstimmung der Diagnostik von niedrig-Risiko-MDS umfassender zu untersuchen. Für die zytologische Diagnose eines RCUD und RARS konnte eine sehr geringe Übereinstimmung von $21 \%$ bzw. $18 \%$ festgestellt werden (Font et al. 2015). Anhand von Knochenmarksaspiraten und Ausstrichen von peripherem Blut haben Senent at al. (2013) 50 Präparate von vier Untersuchern aus drei Zentren evaluiert. Insgesamt zeigte sich eine mäßige Übereinstimmung hinsichtlich der Einteilung in unterschiedliche WHO-Subtypen. Besonders differierten die Befunde bezüglich der Abgrenzung von RAEB-1 sowie RCMD mit Ringsideroblasten. Im Gegensatz dazu konnte in einer Studie mit 103 Patienten mit MDS eine sehr hohe Übereinstimmung (92\%) zwischen drei Untersuchern hinsichtlich der Einordnung in die WHO-Klassifikation belegt werden (Howe 2004). Die bestehende Diskrepanz wurde auch in diesem Kollektiv durch die Schwierigkeit bedingt, RA und RCMD voneinander abzugrenzen. 
Ein weiteres untersuchtes morphologisches Kriterium war der Blastenanteil. Font et al. (2015) untersuchten, inwiefern sich die vom IPSS-R neu einführte Unterscheidung zwischen einem Anteil von $\leq 2 \%$ Blasten und einem von $>2$ bis $<5 \%$ Blasten im Knochenmark umsetzten ließ. Es zeigte sich, dass die Ergebnisse zwischen zwei Untersuchern nicht reproduzierbar waren $($ Kappa Test $=0.277, \mathrm{p}=0,004)$. Diese Erkenntnisse decken sich mit einer Studie von Della Porta et al. (2015), in der gezeigt werden konnte, dass die Interrater-Reliabilität bei Blastenzahlen von unter $5 \%$ abnahm. Auch bei der Untersuchung von Senent et al. (2013) war die Urteiler-Übereinstimmung besonders hoch bei einer Einteilung $<2 \%$ und $>10 \%$ Blasten. Bei der Bewertung einer mäßigen Blastenerhöhung exsistierte ebenfalls eine geringere Übereinstimmung.

Auch einzelne morphologische Kriterien wurden im Hinblick auf die Reproduzierbarkeit untersucht. Die WHO-Klassifikation fordert einen Anteil dysplastischer Zellen von > $10 \%$. Senent et al. (2013) konnten bei der Betrachtung dieses Kriteriums eine mäßige Übereinstimmung bei granulozytären und megakaryozytären Veränderungen und eine schlechte Übereinstimmung bei erythrozytären Veränderungen feststellen. In einer Studie mit 1150 Patienten wurde nach Kriterien gesucht, die eine morphologische Abgrenzung des MDS mit Fibrose (MDS-f) von primärer Myelofibrose (PMF, - eine myeloproliferative Neoplasie mit starken morphologischen Ähnlichkeiten zu einem MDS) sowie des hypozellulären MDS (HypoMDS) von aplastischer Anämie (AA) ermöglichen. Für die im endgültigen Score ausgewählten Kriterien konnte ein sehr hoher K-Koeffizient als Maß für die Reproduzierbarkeit erreicht werden. Je nach Kriterium lag dieser bei der Abgrenzung MDS-f/PMF zwischen 0,84 und 0,95 sowie 0,81 und 0,90 bei der Unterscheidung Hypo-MDS/AA (Della Porta et al. 2015). In dieser Studie wurden sowohl zytologische als auch histologische Kriterien verwendet.

Die angeführten Studien zeigen ein allgemeines Problem der diagnostischen Sicherheit in der Pathologie. Allerdings nehmen die Unterschiede in der morphologischen Beurteilung ab, wenn klare Kriterien vorliegen. Je genauer diese definiert sind, desto besser ist die Reproduzierbarkeit. Aus diesem Grund befassen sich viele Arbeitsgruppen mit minimalen diagnostischen Kriterien bei MDS. Mufti et al. (2008) entwickelten in einer internationalen Konsensusgruppe Vorgaben für die korrekte Ermittlung des Blastenanteils und die Differenzierung von Ringsideroblasten im zytologischen Ausstrich. Bestimmte Dysmorphie-Kriterien für einzelne Zelllinien wurden ebenfalls beschrieben, zum Beispiel von Goasguen et al. (2014) für die Granulopoiese (Reduktion der Granulationen, abnormal segmentierte neutrophile 
Nukleoli, Makropolyzyten, nukleäre Extensionen) und von Kawai et al. (2016) für die Erytrhopoiese (red cell with abnormal chromatin clumping, RCACC).

Inwiefern die in dieser Dissertation gemachten Ergebnisse auch von anderen Untersuchern reproduzierbar sind, ist eine interessante Fragestellung für zukünftige Untersuchungen. Die Tatsache, dass in der vorliegenden Studie ein einzelner Untersucher die histologische Einordnung vorgenommen hatte, entspricht der Praxis, ist jedoch im Hinblick auf die Allgemeingültigkeit der Ergebnisse eine Limitation.

\subsubsection{Genetische Informationen}

Die Aufteilung der genetischen Risikofaktoren im vorliegenden Kollektiv ist vergleichbar mit den Angaben in der Literatur. In der dem IPSS-R zugrunde liegenden Studie zum genetischen Risiko waren die 2902 Patienten folgendermaßen auf die Prognosegruppen verteilt: sehr günstig (2,9\%), günstig (65,7\%), intermediär (19,2\%), ungünstig (5,4\%) und sehr ungünstig (6,8 \%) (Schanz et al. 2012). In der vorliegenden Dissertation war die Aufteilung hinsichtlich der Prognose von sehr günstig bis sehr ungünstig: 1,1 \%, 61,5 \%, 14,3 \%, 9,9\% und 13,2\%. Damit lag der Anteil der Hochrisiko-Genetik (ungünstig und sehr ungünstig) etwas höher, was vermutlich auf den Einschluss von Patienten mit sekundärem MDS zurückzuführen ist.

Im Hinblick auf die verwendeten genetischen Daten ergeben sich verschiedene Limitationen. Zum einen lag nicht für alle Patienten eine Metaphasen-Untersuchung vor, sodass zum Teil auf FISH-Untersuchungen aus dem Knochenmark und aus peripherem Blut zurückgegriffen wurde. Die Gefahr lag darin, dass bestimmte, vom Sonden-Panel nicht abgedeckte Aberrationen unerkannt blieben und somit ein höheres zytogenetisches Risiko vorlag. Bei Betrachtung der genetischen Patientencharakteristika ist die Wahrscheinlichkeit hierfür aber gering. Bei Patienten mit mehr als 3 im FISH nachgewiesenen Aberrationen war keine weitere Verschlechterung des IPSS-R mehr möglich. Bei Patienten mit 3 Aberrationen inklusive der p53Sonde waren weitere Aberrationen wahrscheinlich, sodass der höhere IPSS-R-Score vergeben wurde (4 statt 3). Mit Ausnahme von zwei Patienten (ID21, ID80) war das Risiko durch eine darauffolgende oder vorgegangene Untersuchung zytogenetisch gesichert.

In Bezug auf die FISH-Sensitivität konnte gezeigt werden, dass sie an CD34+-Blasten aus dem Knochenmark (KM) in $96 \%$ die Diagnose der klassischen Chromosomenbänderungsanalyse bestätigte. Ein Zusatznutzen bestand somit nicht, wenn genügend Material für eine Metaphasenanalyse vorlag. Ebenfalls konnte im Vergleich zum Goldstandard Chromosomenbänderungsanalyse eine hohe Sensitivität und Spezifität der FISH an CD34+-Zellen aus 
dem peripheren Blut nachgewiesen werden (Braulke et al. 2013). Ergebnisse dieser Analyse von CD34+-pB-Zellen konnten für die zytogenetischen Risikogruppen nach IPSS-R validiert werden. Somit kann eine durch den Sonden-Panel nachgewiesene Aberration zur Risikoeinschätzung von MDS-Patienten genutzt werden, wenn frühere oder spätere Diagnostik diesen Befund bestätigt (Braulke et al. 2015). Der Vorteil dieser Technik besteht darin, dass dem Patienten die Knochenmarkspunktion erspart werden kann und eine einfache Verlaufskontrolle möglich ist, um Progress oder Therapieansprechen zu beurteilen. Außerdem kann dieses Verfahren angewendet werden, wenn kein Knochenmark aspiriert werden kann (Punctio sicca) oder der Patient in das invasive Verfahren einer Knochenmarkspunktion nicht einwilligt.

Ein weiterer möglicher Faktor für eine verminderte Beurteilbarkeit ist eine zu geringe Anzahl von Metaphasen. In einer Studie mit 529 Patienten haben Steidl et al. (2005) den Informationsverlust mit abnehmender Anzahl an analysierten Metaphasen umfassend untersucht. Empfohlen werden über 20 Metaphasen (Haferlach et al. 2007; Steidl et al. 2005). Bei niedrigerer Anzahl besteht insbesondere bei Angabe einer normalen Zytogenetik, die Gefahr einen kleinen aberranten Klon zu übersehen. In der vorliegenden Arbeit waren von dieser Einschränkung der Sensitivität insbesondere zwei Patienten betroffen (ID23, ID64).

Insgesamt erhöht sowohl die Verwendung der FISH als auch von Karyogrammen mit wenigen Metaphasen die diagnostische Unsicherheit in Bezug auf die Bestimmung des IPSS-R. Allerdings entspricht es dem alläglichen klinischen Vorgehen, anhand der zur Verfügung stehenden Informationen eine Diagnose zu treffen, das Risiko abzuschätzen und eine Therapieentscheidung zu fällen. Daher wurden die Patienten mit ID23 und ID64 nicht ausgeschlossen.

Des Weiteren ist zu diskutieren, inwiefern der IPSS-R als Maßstab für das zytogenetisches Risiko geeignet ist. Nach der aktuellen Revision können 91 \% aller Patienten anhand der im Zytogenetik-Modul abgedeckten Aberrationen klassifiziert werden (Schanz et al. 2012). Seltene, im IPSS-R noch nicht abgebildete Aberrationen werden in die Risikogruppe intermediate mit 2 Punkten einsortiert (Greenberg et al. 2012). Auch diesen können zum Teil schon spezifischen Prognosegruppen zugeordnet werden (Bacher et al. 2014), jedoch sind diese zum Teil günstiger oder ungünstiger als intermediate. Es ist also anzunehmen, dass das tatsächliche Risiko in dieser Prognosegruppe heterogen ist. Eine weitere Herausforderung stellt das genetische Risiko der Patienten mit Aberrationen dar, die nicht mittels FISH oder Karyogramm nachweißbar sind. Nach dem IPSS-R werden diese in die Risikogruppe good (1 Punkt) eingeordnet. Jedoch liegen auch bei diesen Patienten Mutationen vor, die zumeist Splicing- 
Faktoren und epigenetischen Regulatoren betreffen (Bejar und Steensma 2014). So konnten Bejar et al. (2011) Punktmutationen in sechs Genen identifizieren, die bei 29 \% der Patienten mit normalem Karyotyp IPSS-korrigiert zu einem schlechteren Gesamtüberleben führten. In naher Zukunft ist mit der Veröffentlichung eines IPSS-R-molecular zu rechnen, der diese Limitation beheben wird. Diese molekulargenetischen Erkenntnisse stellen die Therapeuten wiederum vor neue diagnostische Herausforderungen, da somatische, mit MDS-assoziierte Mutationen auch bei Patienten ohne hämatologische Grunderkrankung auftreten können eine Entität, die mit dem Begriff clonal hematopoiesis of indeterminate potential (CHIP) umschrieben wird (Steensma et al. 2015).

Zusammenfassend kann festgehalten werden, dass sich die genetische Diagnostik bei MDS aktuell im Wandel befindet. Die Einordnung des genetischen Risikos anhand des IPSS-R ist im vorliegenden Kollektiv die aussagekräftigste verfügbare Methode.

\subsubsection{Patientenkollektiv}

Das Patientenkollektiv war in Bezug auf die Verteilung von Alter und Geschlecht mit demographischen Daten aus ähnlichen Studien vergleichbar. Das erhöhte Risiko von Männern zu Frauen (59 \% zu $41 \%$ ) findet sich auch in größeren Kollektiven ((Della Porta et al. (2015): 318 Patienten mit 58 \% zu 42 \%; Schanz et al. (2012): 2902 Patienten mit 58,4 \% zu 41,6 \%). Die Altersverteilung zeigte die typische Form mit einem mit dem Alter kontinuierlichen Anstieg der Inzidenz (Ma et al. 2007). Auch der Median war mit 71 Jahren vergleichbar mit Angaben in der Literatur (Schanz et al. (2012): 70 Jahre). Alter und Geschlecht wurden in alle Regressionsanalysen eingeschlossen, um für ihren möglichen Effekt zu adjustieren.

Eine Besonderheit der vorliegenden Studie lag im Einschluss von Patienten mit sekundärem, Therapie-assoziiertem MDS in das morphologisch zu beurteilende Kollektiv. In der WHOKlassifikation werden diese zusammen mit einer Therapie-assoziierten AML in einem separaten Abschnitt (therapy-related myeloid neoplasms) erfasst. Aber es wird darauf verwiesen, dass es dem Untersucher überlassen bleibt, ob er die morphologische Klassifikation anwendet. Ebenfalls wurde der IPSS und der IPSS-R für de-novo-MDS entwickelt. Insbesondere unterscheidet sich das t-MDS in Häufigkeit und Risiko der nachgewiesenen Aberrationen. In verschiedenen Studien wurde eine vorhandene zytogenetische Aberrationen in $80-90 \%$ der Fälle nachgewiesen im Gegensatz zu ca. 50 \% bei de novo-MDS (Kantarjian et al. (1986), Pedersen-Bjergaard et al. (1993), Mauritzson et al. (2002), Singh et al. (2007)). Zudem tritt bei t-MDS häufiger eine Hochrisiko-Zytogenetik auf (so zum Beispiel bei Singh et al. 2007: $70 \%$ "poor", 16\% "intermediate" sowie 14\% "good" nach IPSS). In dieser Studie wurde unter 
Einbeziehung von 155 Patienten mit t-MDS untersucht, inwiefern Morphologie und Zytogenetik prognostische Aussagen erlauben. Zwar waren die Überlebenszeiten in den Subgruppen der WHO-Klassifikation deutlich schlechter bei t-MDS im Vergleich zu de novo-MDS in anderen Studien (zum Beispiel t-RCMD vs. de novo-RCMD). Innerhalb dieser WHOGruppen konnte aber in Bezug auf das Gesamtüberleben oder AML-Progression keine Risikostratifizierung anhand der Morphologie (t-RCMD vs. t-RAEB-1 vs. t-RAEB-2 oder $<=$ $5 \%$ vs. $>5 \%$ Blasten) vorgenommen werden. Im Gegensatz dazu konnte für das zytogenetische Risiko (nach IPSS sowie im Vergleich $<3$ oder $\geq 3$ Aberrationen) ein Unterschied im Gesamtüberleben festgestellt werden (Singh et al. 2007). Dies zeigte, dass die Zytogenetik auch bei t-MDS in verschiedene Risikogruppen stratifiziert.

Zusammenfassend weisen die Vergleichsliteratur nach, dass die vorausgegangene Chemooder Radiotherapie ein relevanter Einflussfaktor und eine potentielle Störvariable bei der Untersuchung des Zusammenhangs zwischen Histologie und Zytogenetik sein kann. In der vorliegenden Arbeit wurde dieser potentielle Einflus mit Hilfe von statistischen Methoden überprüft. So war die sekundäre Ätiologie, also eine Therapie-Assoziation, sowohl im Gesamt- als auch im Score-Kollektiv in den Regressionsmodellen enthalten. Sie hatte auch, wie erwartet, einen signifikanten, starken Einfluss, beispielsweise im finalen Modell einen Odds Ratio von 17,65 mit einem 95\%-KI von 2,10 bis 148,64. Somit lag ein kontrollierter Einfluss auf die anderen Prädiktoren vor.

Die letzte Limitation in Bezug auf das Patientenkollektiv entstand durch den Einschluss von Patienten unter Therapie. Es wurde dadurch heterogener. Von der Erhebung des Scores wurden diese Patienten ausgeschlossen, um einen Einfluss auf die Zellmorphologie zu vermeiden.

\subsubsection{Verwendete Statistik}

Um auf einen Zusammenhang zwischen dem zytogenetischen Risiko und den morphologischen Kriterien schließen zu können, wurden die Spearman-Rangkorrelation sowie Regressionsanalysen verwendet. Die Spearman-Rangkorrelation misst einen monotonen Zusammenhang von ordinal skalierten Ratingmerkmalen, wofür sowohl der IPSS-R als auch die erhobenen morphologischen Daten geeignet waren.

Die weiterführende Analyse wurde mit der ordinalen logistischen Regression durchgeführt. In der vorliegenden Studie war die Aussage über eine statistische Signifikanz der Regression dadurch eingeschränkt, dass eine relativ kleine Anzahl von Fällen im Score-Kollektiv $(\mathrm{n}=55)$ einer großen Anzahl von zu betrachtenden morphologischen Kriterien 
gegenüberstand. Bei niedrigen Fallzahlen sind nur sehr starke Effekte signifikant (Faller 2004). Um dem explorativen Ansatz dieser Studie gerecht zu werden, wurden dementsprechend auch Ergebnisse mit einem p-Wert < 0,1 dargestellt und diskutiert. Zur Bestimmung der möglichen klinischen Relevanz - hier bewusst der statistischen Signifikanz gegenübergestellt - sind die Größe der Effektstärke (als Odds Ratio) und die Präzision der Schätzung sowie ihre Lage (als 95\%-KI) besser geeignet und werden deshalb auch bei p-Werten $>0,05$ diskutiert (Faller 2004; Stang et al. 2010; Dick und Tevaearai 2015; Park 2016).

\subsection{Zusammenhang zwischen Histologie und WHO-Kriterien}

Als erstes wurde in dieser Arbeit die Frage behandelt, inwiefern die WHO-Klassifikation auf histologische Präparate (ohne Betrachtung von Knochenmarksausstrichen) anwendbar ist. Dass eine Einteilung in Kategorien mit notwendiger Zytogenetik (MDS mit del(5q) und MDS-U) nicht möglich ist, wurde bereits besprochen. Betrachtet man die Verteilung der histologisch gestellten WHO-Studiendiagnose, so wird klar, dass es eine deutliche Verschiebung hin zu den Kategorien MDS-SLB bzw. MDS-EB-1/2 gab. In einer Studie von Della Porta et al. (2009) mit der zytologischen Untersuchung von 301 Patienten betrug die Aufteilung RA/RARS/MDS del5q zu RCMD/RCMD-RS zu RAEB-1 zu RAEB-2 ca. 34 \% zu 37 $\%$ zu $15 \%$ zu $14 \%$. In der vorliegenden Studie lag die Verteilung bei $3 \%$ vs. $43 \%$ vs. $22 \%$ vs. $32 \%$. Dies ist wahrscheinlich damit zu begründen, dass das von der WHO geforderte Vorhandensein von Dysplasien in $>10 \%$ der Zellen in 2 oder mehr Zellreihen in der histologischen Ansicht schneller erreicht wird. Eine Quantifizierung des Blastengehalts scheint mit Hilfe der CD34- und CD117-Immunhistochemie gut zu gelingen. Es zeigte sich jedoch eine unterschiedliche Häufigkeit der einzelnen Subtypen im Vergleich. In der histologischen Diagnostik war der Anteil der Patienten mit Blastenvermehrung höher als bei der zytologischen Betrachtung. Daraus lässt sich folgern, dass die Abgrenzung einer MDS-SLD von einer MDS-MLD im histologischen Präparat schwierig ist. Möglicherweise sind andere Grenzwerte der Dysplasie sowie Konsens-Kriterien für typische Dysplasiezeichen notwendig. Diese Schwierigkeiten wurden bereits in der alleinigen histologischen Einordnung der FABKlassifikation beschrieben (Horny et al. 1995). 


\subsection{Zusammenhang zwischen Histologie und Zytogenetik}

Ein weiteres Ziel dieser Dissertation war es, zu untersuchen, inwiefern ein Zusammenhang zwischen histomorpholoigsch beobachteten Kriterien und dem zytogenetischen Risiko laut IPSS-R besteht. Im Folgenden werden die verschiedenen Parameter, die im pathologischen Präparat beurteilt werden, mit diesbezüglichen Angaben in der Literatur verglichen.

\subsubsection{Zellularität}

Die Steigerung der Zellularität im Knochenmark bei gleichzeitiger peripherer Zytopenie (ineffektive Hämatopoiese) ist eines der Grundmerkmale des myelodysplastischen Syndroms. Gleichzeitig ist seit längerem eine morphologische Sonderform des MDS bekannt, die mit einem leereren Knochenmark einhergeht. Diesem hypozellulären MDS und seiner Aussagekraft für die Prognose widmet sich auch ein Großteil der Literatur zu diesem Thema, die im Folgenden betrachtet wird.

Bereits in frühen Studien finden sich widersprüchliche Ergebnisse zum hypozellulären MDS. Während bei manchen Autoren (Riccardi et al. 1987; Kitagawa et al. 1989; Ríos et al. 1990) ein günstiger Effekt auf das Überleben und die Zeit bis zur Progression zur Leukämie festgestellt wurde, beobachteten andere keinen Unterschied im Vergleich zu Patienten mit normo- bzw. hyperzellulärem Knochenmark (Yoshida et al. 1988; Toyama et al. 1993; Tuzuner et al. 1995). Aktuelle Arbeiten kommen zu dem Schluss, dass eine Verminderung der Zellularität ein günstiges Merkmal bei MDS darstellt. In einer Untersuchung von Yue et al. (2008) wurden 163 hypozelluläre mit 886 normo- oder hyperzelluärem MDS-Patienten verglichen. Bei einer Einteilung in die drei zytogenetischen Risikogruppen nach IPSS (good, intermediate, poor) ließ sich kein signifikanter Unterschied zwischen hypozellulären und normozellulärem/hyperzellulären Patienten nachweisen. In Bezug auf die Aussagekraft über die Prognose konnte jedoch festgestellt werden, dass die Hypozellularität in allen zytogenetischen Risikogruppen sowie auch in allen Gruppen des IPSS-Gesamtscores mit einem besseren Überleben einherging (Yue et al. 2008). In einer Studie mit 189 Patienten konnten Huang et al (2008) ebenfalls das hypoplastische MDS als einen eigenen Subtyp mit Aussagekraft für die Prognose, besonders in den Gruppen mit low und int-1 nach IPSS, nachweisen. Eine Korrelation mit unterschiedlichen genetischen Risikogruppen stratifiziert mit dem IPSS wurde nicht durchgeführt, es wurden jedoch einzelne Aberrationen untersucht. Ein deutlich selteneres Vorkommen von 7/7q-Aberrationen zeigte sich bei hypozellulärem MDS ( 0 vs. 19 Patienten). Dies stimmte mit Daten von Tuzuner et al. (1995) überein, in denen sich ebenfalls nur in 1 aus 23 Patienten eine solche Aberration nachweisen ließ. Bei Schemenau 
et al. (2015) war 7q- bei Patienten mit hypozellulärem Knochenmark vermehrt, eine Monosomie 7 jedoch bei hyperzellulärem am häufigsten. Diese unterschiedlichen Daten zeigten, dass die Rolle eines spezifischen hypozellulären Phänotyps mit Aberration am Chromosom 7 noch offen ist.

Tong et al. sehen das hypoplastische MDS als eigene Entität an und haben für diese in einer Studie mit 253 hypozellulären und 1725 normo-/hyperzellulären Patienten einen PrognoseScore erarbeitet. Im Hinblick auf die zytogenetische Risikoverteilung nach IPSS zeigte sich in beiden Gruppen kein Unterscheid. Ein längeres Gesamtüberleben wurde für Patienten mit hypozellulärem Knochenmark nachgewiesen (Tong et al. 2012). In einer aktuellen Studie an 1270 Patienten wurde die Zellularität als Kriterium bei MDS untersucht (Schemenau et al. 2015). In einem ersten Schritt wurde der Stellenwert der Histologie in diesem Prozess herausgearbeitet. In einem direkten Vergleich mit der zytologischen Evaluation wurden nur 36,5 \% der hypozellulären Patienten von dieser richtig erkannt. Dies verdeutlichte den Stellenwert der Histologie als Goldstandard zur Beurteilung der Zellularität. Im zweiten Schritt wurde gezeigt, dass sich die Risiken laut IPSS-R signifikant zwischen hypo-, normo- und hyperzellulärem Knochenmark unterschieden. Den niedrigsten Anteil prognostisch ungünstiger Zytogenetik wies die Gruppe mit normozellulärem Knochenmark auf. Ebenfalls konnte eine schlechtere Prognose für Patienten mit hyperzellulärem Knochenmark nachgewiesen werden, was die Autoren auf den höheren Anteil ungünstiger Zytogenetik laut IPSS in dieser Gruppe zurückführen.

Zusammenfassend stimmt die bisher veröffentliche Literatur mit den in dieser Arbeit erhobenen Daten überein. In der Spearman-Rangkorrelation konnte kein Zusammenhang zwischen der alterskorrigierten Zellularität und dem IPSS-R nachgewiesen werden. Auch bei der logistischen Regression lag bei einem Vergleich zwischen hypozellulären und normozellulärem Knochenmark kein signifikanter Zusammenhang im Gesamt- oder Score-Kollektiv vor. Im Gegensatz zu den meisten bisher publizierten Daten war jedoch ein Einfluss von einem hyperzellulären im Vergleich zum normozellulären Knochenmark möglich. Diese Differenzierung wurde bei vergleichbaren Studien außer bei Schemenau et al. (2015) nicht vorgenommen, da hier normo- und hyperzelluläres MDS als eine gemeinsame Gruppe betrachtet wurde. In der vorliegenden Dissertation konnte ein Einfluss der Hyperzellularität unter Einschluss der Granulopoiese und Erythropoiese beobachtet werden, nicht jedoch bei der Megakaryopoiese und den Routineparametern. Im finalen Modell zeigte sich ebenfalls der deutliche potentielle Einfluss dieses Parameters (OR: 10,75; 95\%-KI: 0,88-131,55; p-Wert: 0,06). 
Bei zukünftigen Studien sollte auch der Einfluss einer altersadjustiert gesteigerten Hämatopoiese in die Evaluation mit einbezogen werden.

\subsubsection{Fibrose}

Die Fähigkeit zur Beurteilung des Fibrosegrades ist eines der Alleinstellungsmerkmale der Histologie im Zusammenspiel der verschiedenen Untersuchungsverfahren bei MDS. Die Häufigkeit schwerer Knochenmarksfibrose (MF2/3) lag im Gesamtkollektiv der vorliegenden Studie bei $16 \%$ und im Score-Kollektiv bei $18 \%$. Damit ist sie vergleichbar mit den in der Literatur beschriebenen Ausprägungen von 10 - 20 \% (Lambertenghi-Deliliers et al. 1991; Lambertenghi-Deliliers et al. 1993; Buesche et al. 2008; Della Porta et al. 2009; Fu et al. 2014). Mehrere aktuelle Studien haben den Einfluss dieses Faktors auf die Diagnostik und Prognose untersucht, wobei zum Teil auch Informationen zum genetischen Risiko erhoben wurden.

In einer Studie mit 200 Patienten konnte ein vom Blastenanteil (peripher und im Knochenmark) und der Zytogenetik nach IPSS unabhängiger signifikanter Einfluss der Fibrose auf das Gesamt-Überleben festgestellt werden (Buesche et al. 2008). Das unterschiedliche genetische Risiko für Patienten mit verschieden ausgeprägter Markraumfibrose wurde nicht erhoben. In einer Studie mit 301 Patienten zur Untersuchung von histopathologischen Prognosefaktoren zeigte sich der signifikante Einfluss der Myelofibrose (MF) Grad 2/3 auf das Gesamtüberleben. Ebenfalls erfasst wurde der Zusammenhang zwischen Fibrosegrad und Zytogenetik. In der Patientengruppe mit MF2/3 war die Zytogenetik nach IPSS signifikant schlechter als bei MF0/1. Der Einfluss der Fibrose auf das Gesamtüberleben blieb bestehen, wenn für diese ungünstige Zytogenetik kontrollierte wurde. Dies geschah durch den Einschluss des IPSS-Gesamtscores sowie des WPSS in die multivariate Analyse. Die Autoren konnten durch Berechnung der Hazard Ratios belegen, dass das Vorhandensein einer relevanten Markraumfibrose prognostisch dem Aufstieg in eine höhere Risikogruppe im IPSS oder WPSS entspricht (Della Porta et al. 2009).

In einer weiteren Untersuchung mit 245 Patienten (79 MDS mit Fibrose und 166 ohne Fibrose) wurde der Einfluss dieses Risikofaktors auf Prognose und Therapieansprechen untersucht (Fu et al. 2014). Es wurde ebenfalls eine Analyse des zytogenetischen Risikos in beiden Gruppen durchgeführt. Im Gegensatz zu Della Porta et al. zeigte sich kein signifikanter Unterschied zwischen der Gruppen MF0/1 versus MF2/3 unter Betrachtung des zytogenetischen Subscores des IPSS-R. Bestätigt werden konnte jedoch eine unabhängige prognostische Aussagekraft für das Gesamtüberleben und das leukämiefreie Überleben. In einer 
aktuellen Studie mit 77 Patienten zu den klinisch-biologischen Eigenschaften von MDSPatienten mit Fibrose konnte in der Korrelationsanalyse nach Spearman kein Zusammenhang mit genetischen Risiko nach IPSS-R festgestellt werden (Ramos et al. 2016). In einer weiteren Studie der Arbeitsgruppe von Fu et al. wurde der Einfluss der Markraumfibrose (MF0/1 vs. MF2/3) bei 266 Patienten mit sekundären, Therapie-assoziiertem MDS betrachtet. Die beiden Gruppen unterschieden sich nicht im Hinblick auf die Anzahl von Patienten mit abnormen Karyotyp ( 80 bzw. 82 \%), die Aufteilung in verschiedene zytogenetische Risikogruppen (nach IPSS-R) oder den Anteil von komplexen sowie monosomalen Karyotypen. In dieser Studie wurde zudem zwischen den einzelnen zytogenetischen Aberrationen differenziert. Eine ähnliche Verteilung aller Aberrationen war zu erkennen außer für -5 und -17. Die für primäres MDS nachgewiesene prognostische Aussagekraft der Markraumfibrose war für t-MDS sowohl für das Gesamtüberleben als auch für das leukämiefreie Überleben nicht gegeben (Fu et al. 2013). Bei der Revision des IPSS-R in 2012 wurden über die bestehenden Kriterien (Genetik, Blastenanteil, Zytopenie) hinausgehende Parameter zur Bestimmung des klinischen Verlaufs an einer internationalen Kohorte von 7012 Patienten evaluiert. Es zeigte sich kein zusätzlicher Nutzten des Parameters Fibrose beim Einschluss in den IPSS-R (Greenberg et al. 2012). Die Autoren verweisen darauf, dass dies an der relativen Seltenheit dieses Charakteristikums sowie an nicht standardisierten Fibrosegrad-Einteilungen in früheren Studien liegen kann.

Zusammenfassend konnte in mehreren, aber nicht allen Studien die prognostische Relevanz der Fibrose bei primärem MDS nachgewiesen werden. Zu der Annahme, dass ein teilweiser Zusammenhang zwischen Fibrose und Genetik besteht, liegen bislang nur widersprüchliche Daten vor. In der vorliegenden Dissertation zeigte sich ein deutlicher Zusammenhang sowohl bei den Korrelations- als auch den Regressionsanalysen der Routineparameter, der Granulopoiese sowie in geringerem Maße der Erythropoiese. Im kompletten Modell war nur noch ein schwacher Trend (p-Wert: 0,11) zu erkennen, wobei das Konfidenzintervall immer noch für einen deutlichen möglichen Einfluss sprach (OR: 2,34; 95\%-KI: 0,86; 6,69). In den oben genannten Studien wurde die Unterscheidung zwischen einer Markraumfibrose Grad 0 und 1 vs. Grad 2 und 3 für die Auswertung verwendet. In der vorliegenden Arbeit wurden diese Subgruppen nicht gebildet, sondern die Variable als eine sich kontinuierlich verschlechternde Kategorie aufgefasst. Hierin könnte ein Grund für die teilweise unterschiedlichen Ergebnisse liegen. 


\subsubsection{Siderose}

In den erhobenen Daten in der vorliegenden Arbeit gab es vereinzelte Hinweise auf einen möglichen Einfluss der Markraumsiderose auf das genetische Risiko nach IPSS-R. In der Spearman-Rangkorrelation bestand ein schwacher Zusammenhang. Im Regressionsmodell unter Einschluss der Routineparameter war ein Trend zu erkennen, der dies stützt. Insbesondere der OR wies auf eine deutliche Verlagerung in den Bereich eines gesteigerten Risikos (95\%-KI: 0,93; 2,66). Allerdings war dies unter Einschluss der Erythropoiese, Granulopoiese, Megakaryopoiese sowie im vollen Modell nicht mehr nachzuweisen.

Der Vergleich mit der Literatur überrascht. Viele Studien berichten über den Einfluss der Eisenüberladung des Körpers (insbesondere Leber und Knochenmark) auf die Prognose bei MDS und den möglichen positiven Effekt einer Chelattherapie (Leitch 2007; Rose et al. 2010). Zum Stellenwert der Markraumsiderose in der Biopsie bei der Diagnostik von MDS liegen allerdings keine Vergleichsdaten vor. Beschrieben ist zumindest, dass die Ringsideroblasten (in der WHO-Klassifikation bei RARS oder RCMD-RS) in der Biopsie nicht evaluiert werden können, weil das mitochondriale Eisen nicht durch die Eisenfärbung erfasst wird (Horny et al. 1995). Für die Messung einer Eisenüberladung im Gesamtorganismus werden hingegen unterschiedliche invasive und nicht-invasive Methoden unterschieden, beispielsweise Serumferritin, non-transferrin-bound iron (NTBI), MRT von Leber oder Herz sowie Biopsien (Shenoy et al. 2014).

Aus einer pathophysiologischen Perspektive konnten bereits interessante Erkenntnisse über den Zusammenhang von Eisenüberladung im Knochenmark und der Hämatopoiese mit Hilfe von Mäusemodellen gewonnen werden. So induzierten Okabe et al. (2014) eine Eisenüberladung in Mäusen durch Injektion. Es zeigte sich eine verspätete Entwicklung von Knochenmarkstransplantaten gesunder Mäuse. Ebenfalls wurden herabgesetzte Genexpressionsmuster (CXCL12, VCAM-1, Kit-ligand und IGF-1) und Zytokin-Level (Erythropoietin und Thrombopoietin) nachgewiesen. Chai et al. (2015) beobachteten in einem Mausmodell, dass die Überladung mit Eisen zu einer herabgesetzten Proliferation von hämatopoietischen Stammzellen führt. Bei Eisenüberladung war auch die Menge an ROS (reactive oxygen species) erhöht. Der Zusammenhang ziwschen oxidativem Stress sowie MDS-Prognosegruppe und Überlebenszeit konnte auch in anderen Studien gezeigt werden (Goncalves et al. 2015). Insbesondere für die erythrozytären Vorstufen konnte ein negativer Einfluss der Siderose auf die Proliferation bereits am Patienten nachgewiesen werden (Hartmann et al. 2013).

Ein weiterer, in die Betrachtung miteinzubeziehender Faktor ist der Zusammenhang zwischen MDS-Prognose, Siderose und Transfusionspflichitgkeit. Zum einen führen vermehrte 
Transfusionen zu einer Siderose des Knochenmarks (Cid et al. 2014), zum anderen ist eine Transfusionspflichtikeit ein Zeichen für eine negative Prognose bei MDS (Malcovati et al. 2005). Es ist somit zu untersuchen, inwiefern eine Siderose des Knochenmarks unabhängig von Transfusionen in der Vorgeschichte ein prognostisch relevanter Faktor bei der Prognosebestimmung des MDS ist.

Zusammengefasst ist die Eisenüberladung des Knochenmarks ein in der Histologie bis jetzt wenig betrachtetes Kriterium, für das sich in der vorliegenden Studie Anhaltspunkte für einen Zusammenhang mit einer ungünstigen Zytogenetik zeigten. Die Betrachtung dieses Parameters im Kontext der Chelattherapie und der Prognosebestimmung bei MDS bietet Möglichkeiten für zukünftige Untersuchungen. Hierbei ist inbesondere der Einfluss einer transfusions-unabhängigen Siderose des Knochenmarks zu betrachten.

\subsubsection{CD34 ${ }^{+}$-Zellen}

Bereits 1984 wurde die Reaktivität des Anti-My-10 monoklonalen Mäuseantikörpers mit hämatopoietischen Vorläuferzellen beschrieben, ohne dass die Zielstruktur bekannt war (Civin et al. 1984). Diese konnte als Glycophosphoprotein identifiziert werden, das an der Oberfläche von hämatopoietischen Stamm- und Vorläuferzellen, embryonischen Fibroblasten und Endothelzellen exprimiert wird (Krause et al. 1996). Bei gesunden Probanden beträgt der Anteil von $\mathrm{CD}_{3} 4^{+}$-Zellen ca. 1 \% der hämatopoietischen Zellen im Knochenmark (Soligo et al. 1991). Infolge der Enddeckung dieses Markers wurden zahlreiche Studien über dessen prognostische Wertigkeit durchgeführt, wobei eine Korrelation mit zytogenetischen Veränderungen selten erfolgte.

In einer Studie mit 35 Patienten wurden verschiedene Antigene (CD34, CD13, CD14, CD15 und CD33) bezüglich ihrer Aussage über das Überleben getestet. Hierbei ergaben sich nur für CD34 relevante Ergebnisse (Guyotat et al. 1990). Der negative Einfluss von CD34+Zellen auf das Gesamtüberleben und das leukämiefreie Überleben konnte an einem Kollektiv von 62 Patienten im peripheren Blut nachgewiesen werden (Sullivan et al. 1992). Dass dies eine valide Methode ist, um den CD34+-Anteil von Zellen im Knochenmark zu untersuchen, konnte ebenfalls nachgewiesen werden. Die CD34-Expression in der histologischen und durchflusszytometrischen Analyse korrelierten gut miteinander (Kanter-Lewensohn et al. 1996). In einer Studie mit 68 MDS-Patienten war das Überleben in der Gruppe mit einem Anteil an CD34 ${ }^{+}$-Blasten $>1 \%$ signifikant kürzer. Außerdem konnte die CD34-Expression in der Patienten-Gruppe mit RAEB eine Subgruppe mit schlechterer Prognose identifizieren (Soligo et al. 1994). In einer Arbeit mit 100 MDS-Patienten konnte sowohl für eine Erhöhung 
von $\mathrm{CD}_{34}{ }^{+}$-Blasten über $1 \%$ als auch für ihre Tendenz, Aggregate zu bilden, ein erniedrigtes Gesamtüberleben sowie eine erhöhte Rate an Leukämien beobachtet werden (Oriani et al. 1996). Eine ungünstigere Prognose bei einer Erhöhung von CD $34^{+}$-Blasten konnte in einer Untersuchung von 17 Patienten mit sekundären, Therapie-assoziiertem MDS ebenfalls gezeigt werden (Orazi et al. 1993).

Außerdem konnte für die CD34-Immunhistochemie eine differentialdiagnostische Bedeutung nachgewiesen werden. In einer Studie an 561 Knochenmarksbiopsien, denen unterschiedliche hämatologische Erkrankungen zugrunde lagen, war der CD34 ${ }^{+}$-Blastenanteil nur bei MDS sowie AML erhöht. Innerhalb der FAB-Klassifikation konnte der Marker außerdem zu einer Abgrenzung von RA/RARS (keine Expression) von RAEB/ RAEB-T (vorhandene Expression) genutzt werden (Horny et al. 1995). Diese Bedeutung wurde in einer aktuellen Studie bestätigt, in der unter Einbeziehung von 1150 Patienten mit Zytopenien differentialdiagnostisch aussagekräftige Parameter untersucht wurden. Das Vorhandensein von $>5 \% \mathrm{CD} 4^{+}$-Zellen sowie deren Cluster-Bildung half bei der Abgrenzung des MDS mit Fibrose von primärer Myelofibrose (PMF) sowie dem hypozellulären MDS von der aplastischen Anämie (AA) (Della Porta et al. 2015).

Oertel et al. untersuchten ebenfalls den prognostischen Einfluss der CD34Immunhistochemie. In einer Studie mit 16 Patienten konnte nachgewiesen werden, dass Patienten mit vermehrten $\mathrm{CD} 34^{+}$-Zellen ein erhöhtes Risiko für eine Progression zur Leukämie hatten (Oertel et al. 1993). Insbesondere bei Patienten mit insgesamt niedrigeren Blastenzahlen (nach FAB) wurde ein niedrigerer $\mathrm{CD}^{+} 4^{+}$-Anteil der Blasten im Vergleich zu allen Blasten gefunden. Dieser Anteil stieg in höheren Stadien (RAEB-T nach FAB-Klassifikation) (Oertel et al. 1994). Eine Zusammenführung dieser beiden Kollektive verdeutlichte, dass die CD34-Immunhistochemie nur einen Anteil aller im Mark vorhandenen Blasten anfärbt. Dieser Anteil stieg aber in höheren Stadien und war dann mit einer schlechteren Überlebenswahrscheinlichkeit assoziiert (Oertel und Huhn 1994).

Eine neuere Studie widerspricht der Ansicht, die CD34-Immunhistochemie färbe nur einen Teil der Blasten im Knochenmark an. Bauer et al. (2000) fanden in einer Untersuchung von 150 Knochenmarksbiopsien einen guten Zusammenhang zwischen morphologisch beobachteten Blasten (in Biopsie und Aspirat) sowie CD34+-Zellen im Knochenmark. Der Anteil der CD34+-Zellen war sogar etwas höher, was auf eine Mitanfärbung früher Promyelozyten zurückgeführt wurde. Bei fünf Patienten war die Anzahl der CD34+-Blasten deutlich geringer als die morphologisch beobachteten. Eine Korrelation mit der Prognose wurde in dieser Studie nicht durchgeführt. Aber ein in höheren FAB-Subgruppen ansteigender Anteil 
$\mathrm{CD} 34^{+}$-Blasten sowie die vermehrte Cluster-Bildung konnte festgestellt werden (Baur et al. 2000). Diese Studie ist eine der wenigen, die eine Korrelation des Blastenanteils mit der Zytogenetik durchgeführt hat. Es zeigte sich ein vermehrtes Auftreten von CD $34^{+}$-Clustern bei Patienten mit doppelten oder komplexen Aberrationen (was einer höheren genetischen Risikogruppe laut IPSS-R entspräche) als in Patienten mit einfacher oder gänzlich ohne Aberration. Der prozentuale Anteil an $\mathrm{CD} 34^{+}$-Blasten korrelierte nicht mit dem zytogenetischen Risiko (Baur et al. 2000).

Ferner untersuchte eine Studie von Della Porta et al. (2009) an 301 Patienten mit MDS unter anderem die Bedeutung des prozentualen Anteils der CD34 ${ }^{+}$-Zellen sowie deren Clusterbildung für die Prognose. In der multivariablen Analyse zeigte sich ein von der WHOSubgruppe, der Zytogenetik (nach IPSS) und der Transfusionsabhängigkeit unabhängiger Effekt von $\mathrm{CD}_{3} 4^{+}$-Clustern auf das Gesamtüberleben und das leukämiefreie Überleben. Dies konnte für den prozentualen Anteil CD34+-Zellen nicht nachgewiesen werden. Eine explizite Korrelation mit dem zytogenetischen Risiko wurde nicht durchgeführt (Della Porta et al. 2009). Die in vorherigen Studien beschriebene Relevanz der Clusterbildung wurde somit bestätigt (Oriani et al. 1996; Baur et al. 2000).

In der bestehenden Literatur konnten nur wenige Anhaltspunkte für einen Zusammenhang zwischen $\mathrm{CD} 4^{+}$-Blasten und der Zytogenetik gefunden werden. Eine Clusterbildung dieser Vorläuferzellen schien jedoch mit einem erhöhten zytogenetischen Risiko assoziiert zu sein. Über die Kontroverse zu einer möglichen Ähnlichkeit von CD34 ${ }^{+}$-Clustern und dem histologischen Kriterium der ALIP wird noch eingegangen (Kapitel 4.3.6.1). Die wenigen vorliegenden Vergleichsdaten stützen den in dieser Arbeit festgestellten fehlenden Zusammenhang zwischen dem prozentualen Anteil an $\mathrm{CD} 34^{+}$-Blasten und dem zytogenetischen Risiko laut IPSS-R.

\subsubsection{Lymphozyten}

Das Vorhandensein von Lymphozytenaggregaten ist ein regelmäßiges Phänomen in der Beurteilung von Knochenmarksbiospien. Unterschieden wird die maligne Knochenmarksinfiltration, beispielsweise durch ein Lymphom, von benignen Lymphozytenaggregaten, wie sie auch in der vorliegenden Studie erfasst wurden (Thiele et al. 1999). In der Literatur gibt es keine eindeutige Klarheit bezüglich ihrer Bedeutung, jedoch zeigen sie eine Korrelation mit bestimmten klinisch-pathologischen Charakteristika in gemischten Kollektiven genauso wie beim MDS. 
Bei einer Untersuchung von 2474 Knochenmarksbiopsien wurden Lymphozytenaggregate in 13,3 \% der Fälle gefunden. Sie waren häufiger bei Frauen, älteren Patienten sowie bestimmten rheumatologischen und hämatologischen Erkrankungen (rheumatoide Arthritis, systemischem Lupus erythematodes, Polycythaemia vera, Immunthrombozytopenie) zu beobachten Magalhães et al. (2002) haben die Charakteristika von Lymphozytenaggregaten bei MDS an einem Kollektiv von 206 Patienten untersucht. Die Patienten mit Lymphozytenaggregaten (24,7 \% der Fälle) zeigten ein niedrigeres Hämoglobin, einen höheren Neutrophilenanteil sowie eine Faservermehrung. Insbesondere die letztere Erkenntnis führte die Autoren zu der Annahme, dass das Vorhandensein von Lymphozytenaggregaten könne mit einem gestörten Mikroklima im Knochenmark einhergehen, bedingt durch immunologische Prozesse. In der Studie fand sich kein Einfluss der Lymphozytenaggregate auf die Prognose. Eine Korrelation mit der Zytogenetik wurde nicht durchgeführt. Ebenso wenig wurde bei den Patienten ein erhöhtes Risiko für lymphoproliferative Erkrankungen festgestellt (Magalhães et al. 2002). Dies steht in Widerspruch zu einer früheren Studie an gesunden Patienten, bei denen das Vorhandensein von benignen Lymphozytenaggregaten mit einem erhöhten Risiko einherging (Faulkner-Jones et al. 1988). Möglicherweise haben Aggregate bei gesunden Menschen eine andere Bedeutung als bei MDS-Patienten. Das Vorhandensein von Lymphozyten bei MDS wurde in einer zytologischen Durchsicht von 3156 Patienten bestätigt. In $16 \%$ der Fälle wurden Lymphozyten und in $13 \%$ Plasmazellen nachgewiesen. Eine Korrelation mit anderen klinischen Parametern, der Zytogenetik oder der Prognose wurde nicht durchgeführt. Die Autoren ziehen als Begründung für das vermehrte Auftreten der Lymphozyten ebenfalls entweder das Alter der Patienten oder ein gestörtes Mikroklima im Knochenmark in Erwägung (Germing et al. 2012).

In der vorliegenden Arbeit zeigte sich lediglich im Regressionsmodell unter Einschluss der Megakaryopoiese ein Trend bezüglich eines Einflusses der Lymphozyten auf das zytogenetische Risiko. Das Vorhandensein einer Hochrisiko-Zytogenetik kann man aus ihrem Nachweis, auch unter Einbezug der Literatur, somit nicht ableiten.

\subsubsection{Dysgranulopoiese}

In der vorliegenden Arbeit konnte gezeigt werden, dass für die Reifungs- und Architekturstörungen der Granulopoiese kein Zusammenhang mit dem IPSS-R besteht. Der in der Korrelationsanalyse festgestellte Zusammenhang zwischen quantitativen Veränderungen der Granulopoiese wurde in der Regression bestätigt. Eine Vermehrung der Granulopoiese hat einen protektiven Effekt auf das genetische Risiko. In der Literatur untersucht ein Großteil der Studien die Relevanz von ALIP, sodass dieses Thema in dieser Arbeit in einem separaten 
Abschnitt besprochen wird. Andere Studien betrachten nur Teilaspekte der Beziehung zwischen Granulopoiese, Genetik und Prognose.

Ein spezieller Zusammenhang wurde zwischen der Genetik (7p-Deletion) und der Dysgranulopoiese in der zytologischen Morphologie (Pseudo-Pelger-Hüet Hypolobulierung und kleine Vakuolen der Neutrophilen) bei Therapie-assoziierten Neoplasien beschrieben. In einer Auswertung der Ergebnisse von 21 Patienten mit t-MDS sowie von 4 Patienten mit tAML (alle mit 7p-Deletion) konnte dies in 92\% der Fälle beobachtet werden (Merlat et al. 1999).

Die meisten anderen Studien widmen sich dem Stellenwert der Dysgranulopoiese bei der zytologischen Evaluation. In einer Publikation wurde die Notwendigkeit herausgestellt, reproduzierbare Kriterien für eine dysplastische Granulopoiese laut WHO-Klassifikation zu erarbeiten. Zu diesem Zweck wurden an 98 Fotos von Granulozyten in der Zytologie minimale diagnostische Kriterien erarbeitet. Eine Korrelation mit Genetik oder Prognose war nicht erfolgt (Goasguen et al. 2014). Bei der Untersuchung des Düsseldorfer MDS-Registers mit 3156 Patienten wurde in der univariaten Analyse ein Zusammenhang zwischen verschiedenen zytologischen Kriterien und dem Gesamtüberleben festgestellt (Hyperplasie, Linksverschiebung, Hypogranulierung, Pseudo-Pelger-Zellen), der jedoch unter Einschluss anderer Kriterien (Blastenanteil, Genetik, LDH) nicht mehr nachzuweisen war. Eine Korrelation dieser Parameter mit der Zytogenetik wurde nicht durchgeführt (Germing et al. 2012). Im Kollektiv von Della Porta et al. mit 1150 Patienten zur Bestimmung minimaler zytologischer Kriterien bei MDS wurde ebenfalls die Granulopoiese betrachtet. Für den Gesamtscore dysplastischer Veränderungen (Myeloblasten, Auer-Stäbchen, Pseudo-Pelger-Huet-Zellen, abnorme Nukleusform, neutrophile Hypogranulation) fand sich eine signifikante Korrelation mit dem zytogenetischen Risiko laut dem MDS yytogenetic scoring system (Della Porta et al. 2015). Dies steht in teilweisem Widerspruch zu den Ergebnissen der vorliegenden Dissertation, in der sich für histologisch beobachtbare zelluläre Veränderungen (Linksverschiebung, GröBenvariabilität) kein diesbezüglicher Zusammenhang nachweisen ließ. Zudem konnte im Gegensatz zu den Beobachtungen von Germing et al. (2012) für den Gesamtscore der zytologischen Veränderungen der Granulopoiese bei Della Porta ein von anderen Einflussfaktoren unabhängiger prognostischer Effekt ausgemacht werden (Hazard Ratio: 3,26; p < 0,001).

In einer neueren histologischen Studie wurde der Einfluss einer Expression von lymphoid enhancer-binding factor 1 (LEF1) bei Patienten mit MDS untersucht (Pellagatti et al. 2009). Die wichtige Funktion von LEF-1 in der Differenzierung granulozytärer Vorstufen war zuvor bereits beschrieben worden, ebenso wie sein pathophysiologisch relevantes Fehlen bei 
kongenitaler Neutropenie (Skokowa et al. 2006). Pellagatti et al. konnten an 122 MDSPatienten und 16 gesunden Kontrollen mit Hilfe eines Micro-Arrays zeigen, dass die LEF-1Genexpression mit zunehmendem Blastenanteil abnahm (Methodik beschreiben in Pellagatti 2006). Auch die doppelte Immunhistochemie von CD34/LEF-1, die für insgesamt neun Patienten (3 Kontrollen, 3 RA und 3 RAEB) durchgeführt wurde, wies eine abnehmende Expression in höherem WHO-Stadium auf. Ebenso konnte ein Einfluss auf das Überleben beobachtet werden, sodass die Autoren annahmen, dass es sich bei einer verminderten LEF1 Expression um einen Progressionsmarker handeln könnte.

In Bezug auf die in der vorliegenden Arbeit untersuchten quantitativen Veränderungen der Granulopoiese konnte ein deutlicher protektiver Effekt einer Zellvermehrung auf das zytogenetische Risiko festgestellt werden (Odds Ratio 0,09; 95\%-KI 0,02; 0,51). Dieser bestätigte sich im vollen Regressionsmodell. Vergleichbare Daten wurden am ehesten von Mangi et al. (1991) erhoben. Hier zeigte sich jedoch eine Subgruppe von Patienten mit ALIP und granulozytärer Hyperplasie, die im Gegensatz zu den in der vorliegenden Dissertation gemachten Beobachtungen eine schlechtere Prognose hatte. Eine Korrelation mit dem genetischen Risiko wurde von Mangi et al. nicht durchgeführt.

Insgesamt betrachtet kann festgehalten werden, dass wenige Vergleichsdaten hinsichtlich des Zusammenhangs der Granulopoiese mit dem IPSS-R vorliegen. Diejenigen aus der zytologischen Beurteilung (Della Porta et al. 2015) widersprechen den in dieser Arbeit gemachten Beobachtungen. Die prognostische Aussagekraft der Dysgranulopoiese ist ungeklärt. In Bezug auf die Diagnosestellung wurden in letzter Zeit einige Anstrengungen für eine Vereinheitlichung der Kriterien unternommen (Goasguen et al. 2014, Della Porta et al. 2015).

\subsubsection{ALIP}

Die "abnormal localization of immature precursors" (ALIP) waren eines der ersten histopathologischen Kriterien, für das eine prognostische Aussagekraft bezüglich MDS beschrieben werden konnte. Tricot et al. (1984b) wiesen an einem Kollektiv von 40 Patienten bei der Anwesenheit von ALIP ein schlechteres Überleben und eine höhere Rate von AMLTransformationen nach. Dies war unabhängig vom zytologischen Subtyp (nach FABKlassifikation) sowie der Zytogenetik (gemessen am Vorhandensein vs. nicht-Vorhandensein von Aberrationen) (Tricot et al. 1984b). In diesem Kollektiv waren ALIP definiert als Myeloblasten oder Promyelozyten, die sich zentral im Knochenmark und nicht mehr entlang der Trabekel befinden. Sie können entweder als Cluster (3-5 Zellen) oder Aggregate (> 5 Zellen) vorliegen. Liegen drei oder mehr dieser Cluster oder Aggregate in einem Präparat vor, so wurde dieses als ALIP+ bezeichnet (Tricot et al. 1984a). 
Seit dieser erstmaligen Beschreibung wurde sowohl über die genaue morphologische Definition als auch über den diagnostischen und prognostischen Wert von ALIP diskutiert. Diagnostisch wird das Erkennen dadurch erschwert, dass es ähnliche Aggregate aus erythrozytären oder megakaryozytären Vorstufen gibt, die von der eigentlichen ALIP der Granulopoiese schwer abzugrenzen sind. Mangi et al. versuchten mittels Immunhistochemie mehr Sicherheit in der Unterscheidung von pseudo-ALIP und ALIP zu generieren (Mangi et al. 1991; Mangi und Mufti 1992). In der Studie von Mangi und Mufti (1992) machten pseudo-ALIP 55 \% (8/14) der Fälle aus. Die Verwendung von immunhistochemischen Markern konnte helfen, eine Gruppe von Patienten mit Hyperplasie von granulozytären Vorstufen und ALIP zu identifizieren, die eine schlechtere Prognose hatten, unabhängig von der FAB-Klassifikation. Eine Korrelation mit zytogenetischen Veränderungen wurde nicht durchgeführt (Mangi und Mufti 1992). Grundsätzlich wird die Diagnostik von ALIP auch dadurch erschwert, dass eine von den Trabekeln entfernte, "abnorme" Lokalisation in einem zweidimensionalen Schnitt gar nicht mit letzter Sicherheit nachgewiesen werden kann (Horny et al. 2007). Dies ist dadurch bedingt, dass die Trabekel knapp außerhalb der Schnittebene des Präparates liegen können und so eine mittig im Markraum befindliche frühe Granulopoiese vorgetäuscht wird.

In einer Studie zur prognostischen Wertigkeit histopathologischer Kriterien mit 126 Patienten mit MDS bestand bei 1207 erhobenen Biopsie-Daten in der ALIP-Kategorie die größte Diskrepanz zwischen den Ergebnissen zweier Untersucher (bei $17 \%$ der Patienten). Auch konnte kein prognostischer Einfluss einer ALIP nachgewiesen werden. Eine Korrelation mit der Zytogenetik wurde nicht durchgeführt (Ríos et al. 1990). Auch in einer Studie zur Bestimmung von prognostisch relevanten histologischen Kriterien bei MDS mit 301 Patienten wurde im Vergleich zu anderen Parametern (Fibrose, CD34+-Blasten, Zellularität) eine schlechte Urteiler-Übereinstimmung festgestellt. Deswegen wurde dieser Parameter von der weiteren Analyse ausgeschlossen (Della Porta et al. 2009). Im Gegensatz hierzu konnte in einer Studie mit 184 Patienten gezeigt werden, dass durch das Vorhandensein von ALIP, insbesondere innerhalb der Risikogruppen Low, INT-1 und INT-2 des IPSS-Gesamtscores, zusätzliche prognostische Informationen gewonnen werden können. Eine Korrelation zwischen dem Vorkommen und dem zytogentischen IPSS-Subscore wurde nicht durchgeführt (Verburgh et al. 2003).

Ein weiteres diskutiertes Problem in der Diagnostik von ALIP ist, ab welcher Größe der Zellansammlung eine prognostische Aussage gemacht werden kann. In einer Studie mit 106 MDS-Patienten wurde eine sich von Tricot et al. unterscheidende Einteilung der blastären 
Infiltrate vorgenommen. Große Ansammlungen ( $>6$ Zellen) hatten einen signifikanten Einfluss auf das Gesamtüberleben, anders als diffuse (ohne Aggregation) oder Cluster ( $\leq 6$ Zellen) (Lambertenghi-Deliliers et al. 1993). Nicht zuletzt mit dem Aufkommen der Immunhistochemie konnte festgestellt werden, dass die Anwesenheit von CD $34^{+}$-Zellen sowie deren Clusterbildung prognostisch ungünstige Verläufe anzeigen (Oriani et al. 1996; Baur et al. 2000; Della Porta et al. 2009). Aufgrund der Ähnlichkeit von ALIP und CD34+-Clustern wurde angeregt, letztere als reproduzierbareres Kriterium zu verwenden, um die ursprünglich für ALIP beschriebene prognostische Einordnung vorzunehmen (Oriani et al. 1996; Lambertenghi-Deliliers et al. 1998). Aus diesem Grund befürworten aktuellere Empfehlungen den Begriff ,abnormal mulitfocal acuulumulation (clustering) of CD $34^{+}$percursor cells" (AMA-CD34) (Horny et al. 2007; Valent et al. 2007; Valent et al. 2010).

Insgesamt wurden hinsichtlich der Verwendung einer ALIP als diagnostischem Kriterium für MDS vielfältige Probleme beschrieben. Diese reichen von der Abgrenzung von ähnlichen morphologischen Strukturen („,pseudo-ALIP“) über die Feststellung einer prognostisch relevanten Größe der Aggregate bis zu einer schlechten Reproduzierbarkeit der Ergebnisse bei verschiedenen Untersuchern. Zu einem Zusammenhang zur Zytogenetik existieren keine aktuellen Vergleichs-Daten. In der vorliegenden Studie konnte keine Korrelation mit dem IPSS-R oder Laborparametern festgestellt werden. Ob ALIP eine von der Genetik unabhängige Rolle für die Prognose spielen, ist entsprechend der widersprüchlichen Ergebnisse weiterhin offen. Wahrscheinlich wird die prognostische Aussagekraft durch die CD34Immunhistochemie besser und reproduzierbarer abgebildet.

\subsubsection{Dyserythropoiese}

Mit Hilfe der in der vorliegenden Studie erhobenen Parameter wurde kein Zusammenhang zwischen Veränderungen der Erythropoiese (Makrozytose, Kerngrößenschwankung, unscharfe Nester) und dem genetischen Risiko laut IPSS-R festgestellt. Insgesamt gibt es wenige Vergleichsdaten zu dieser Thematik.

In einer aktuellen Studie wurde nach zytomorphologischen diagnostischen Kriterien für eine Dyserythropoiese gesucht, indem Präparate von 109 MDS-Patienten mit solchen von 33 Patienten mit ITP bzw. hämolytischer Anämie verglichen wurden. Ein Einfluss dieser Parameter auf die Prognose wurde nicht untersucht, ebenso wenig wie ein Zusammenhang zur Genetik (Kawai et al. 2016). In einer Studie von Della Porta et al. (2015) zur Evaluation von minimalen morphologischen Kriterien für MDS mit 1150 Zytopenie-Patienten wurde auch die Erythropoiese untersucht. Verschiedene erythrozytäre Dysplasien waren bei Patienten 
mit MDS häufiger vertreten als bei Patienten mit anderen Erkrankungen. Außerdem wurden die Veränderungen der Erythropoiese mit dem MDS cytogenetic scoring system (Schanz et al. 2012) verglichen. Hierbei zeigte sich keine signifikante Korrelation (Della Porta et al. 2015). Diese zytomorphologischen Daten stützen den in der vorliegenden Arbeit beobachteten, fehlenden Zusammenhang zwischen genetischen Veränderungen und einer morphologisch dysplastischen Erythropoiese.

In der vorliegenden Arbeit zeigte sich jedoch ein Zusammenhang zwischen Veränderungen der Erythropoiese und den Laborparametern. So ging eine quantitative Steigerung der roten Vorstufen im Knochenmark mit erhöhtem Hämoglobin, Hämatokrit sowie Erythrozytenzahlen einher. Es ist zu vermuten, dass es sich hierbei um eine kompensatorische Steigerung handelt, die in dieser Form bei der Granulopoiese und der Megakaryopoiese nicht zu beobachten war.

\subsubsection{Dysmegakaryopoiese}

Morphologische Veränderungen der Megakaryopoiese sind in einer Vielzahl von Studien als diagnostisch und prognostisch hilfreich bei der Untersuchung von Patienten mit MDS beschrieben worden. Angaben zu einem Zusammenhang mit dem genetischen Risiko sind allerdings selten.

In einer Studie mit 126 Patienten mit MDS zur prognostischen Wertigkeit histopathologischer Kriterien wurde das Vorhandensein einer Dysmegakaryopoiese als ungünstiger Einflussfaktor beschrieben, unabhängig vom FAB-Stadium. Eine Korrelation mit genetischen Analysen wurde nicht durchgeführt (Ríos et al. 1990). Thiele et al. (1991) waren in der Lage, bei 40 Patienten anhand einer ausgeprägten Mikromegakaryozytose sowie einer Vermehrung der Megakaryopoiese eine diagnostische Abgrenzung von normalem Knochenmark sowie von der chronischen myeloischen Leukämie (CML) durchzuführen. Auch zeigte sich in dieser Studie der Vorteil einer CD61-Immunhistochemie: Pro mm² wurden so 69,9 Megakaryozyten statt 30,4 in der PAS-Färbung ausgezählt. Eine Korrelation dieser histologischen Parameter mit der Zytogenetik oder der Prognose wurde nicht durchgeführt (Thiele et al. 1991). Der diagnostische Nutzen der Immunhistochemie wurde in einer Serie mit 40 MDSPatienten und 10 gesunden Kontrollpatienten bestätigt: eine anhand der HE-Färbung festgestellte Verminderung der Megakaryopoese musste nach Einsatz der Immunhistochemie (CD61) in eine Vermehrung revidiert werden. Ohne Verwendung dieser Technik werden insbesondere mikrozytäre Formen leicht übersehen (Das et al. 2005). Die Anzahl der Megakaryozyten wurde in der vorliegenden Studie anhand der CD61-Färbung ermittelt. 
In einem Vergleich von 12 gesunden und 12 an MDS erkrankten Patienten konnte nachgewiesen werden, dass die Kernanzahl in der Kontrollgruppe gleich war (16 Kerne bei allen Kontroll-Patienten), während sie bei den Patienten mit MDS erniedrigt war (10 Patienten mit 8, und 2 mit 4 Kernen). Eine Korrelation mit zytogenetischen Veränderungen wurde nicht durchgeführt (Kobayashi et al. 1991). Die Erkenntnisse wurden in einer Folgestudie auf die Untersuchung von Mikromegakaryozyten angewendet. Es zeigte sich, dass diese sowohl bei gesunden als auch bei MDS-Patienten mit unterschiedlicher Ploidie vorkommen. Die Anzahl der Kerne lag in der Kontrollgruppe durchweg bei 16 und in der MDS-Gruppe bei 4 bzw. 8. Dies unterstreicht die Ausreifungsstörung bei MDS und die Bedeutung eines gemeinsamen Vorliegens von Mikrozytose und verminderter Ploidie (Kobayashi et al. 1995).

Die differentialdiagnostische Aussagekraft der Megakaryopoiese wurde in einer Studie mit 201 Patienten mit unterschiedlichen hämatologischen Erkrankungen (20 gesunde Kontrollen, 94 MDS, 29 MPS, 39 AA, 10 ITP, 9 CML) morphometrisch untersucht (Anzahl der Zellen, Anordnung der Zellen, Nuleusgröße, Nukleus-Plasma-Relation, Nukleus-Kontur-Index). Ein für MDS typischer Phänotyp mit mikrozytären und mononukleären Megakaryozyten konnte identifiziert werden, der differentialdiagnostische Aussagekraft besitzt. Eine Korrelation mit dem zytogenetischen Risiko wurde nicht durchgeführt (Ohshima et al. 1995). Der diagnostische Wert der Dysmegakaryopoiese konnte auch von Li und Pu (2005) in einer Studie mit 29 MDS-Patienten und 14 Gesunden (Kontrolle) nachgewiesen werden. Die Vermehrung von Megakaryozyten und Mikromegakaryozyten war ein signifikantes Unterscheidungsmerkmal gegenüber der Kontrollgruppe. Ebenfalls beschrieben wurde eine atypische Verlagerung von Megakaryozyten an die Knochentrabekel. Eine Korrelation mit dem genetischen Risiko oder der Prognose wurden nicht durchgeführt (Li und Pu 2005).

In der bereits erwähnten Studie mit 1150 Patienten zur Bestimmung minimaler diagnostischer Kriterien bei MDS wurde die Megakaryopoiese zytologisch untersucht und mehrere morphologische Parameter für die MDS-Diagnose eruiert (Della Porta et al. 2015). Von diesen zeigte keiner einen statistischen Zusammenhang mit dem MDS cytogenetic scoring system (Schanz et al. 2012). Allerdings konnte unter Einschluss von Laborparametern, Blastenanteil, demographischen Faktoren sowie der Zytogenetik ein unabhängiger Einfluss der Megakaryopoiese auf das Überleben festgestellt werden.

Ein morphologisches Kriterium der Megakaryopoiese, das in letzter Zeit einige Beachtung erhalten hat, ist die Expression von CD34 in Megakaryozyten als Zeichen einer Differenzierungsstörung. In einer Studie half die Bestimmung der CD34-Expression, MDS von anderen neoplastischen Erkrankungen diagnostisch abzugrenzen (Pellegrini et al. 2000). In anderen 
Studien half dieses Kriterium nicht (Torlakovic et al. 2002) oder nur bei der Abgrenzung von nicht-neoplastischen Veränderungen (Insuasti-Beltran et al. 2012). Unabhängig von der differentialdiagnostischen Aussagekraft konnte bei einem Vorliegen dieser Veränderung bei MDS ein Einfluss auf die Prognose und das genetische Risiko beobachtetet werden. In einer Studie mit 202 Patienten mit MDS wurde eine "positive Gruppe", in der mehr als $20 \%$ der Megakaryozyten CD34 exprimieren, und eine "negative Gruppe" mit weniger als $20 \%$ unterschieden (Tang et al. 2011). Ein signifikant häufigeres Vorkommen von Aberrationen und prognostisch ungünstigen Veränderungen nach IPSS zeigte sich in der Gruppe mit CD34 ${ }^{+}$ Megakaryozyten. Ebenfalls nachgewiesen werden konnte ein vom IPSS-Gesamtscore unabhängiger Einfluss auf die Prognose mit einer Hazard Ratio von 2,53 bei CD34- vs. CD $34^{+}$Megakaryozyten auf das Gesamtüberleben.

Zusammenfassend zeigte sich die diagnostische und prognostische Wertigkeit einer morphologischen Beurteilung der Megakaryopoiese. Bezüglich der Aussagekraft hinsichtlich einer ungünstigen Zytogenetik ergab sich unter Berücksichtigung der wenigen vorliegenden Vergleichsdaten eine teilweise Übereinstimmung. Für histologisch zu beurteilende zelluläre Veränderungen (Hypolobulierung, Mikrozytose) zeigten sich wie auch in den zytologischen Beurteilungen keine Zusammenhänge (Della Porta et al. 2015). Der in anderen Studien beschriebene Einfluss einer CD34-Expression konnte in der vorliegenden Arbeit nicht nachgewiesen werden. Dies könnte daran liegen, dass bei der vorliegenden Dissertation eine Gradeinteilung von 0 bis 3 verwendet wurde, in anderen Studien jedoch eine Unterteilung in $<$ versus $>20 \%$ Expression (Tang et al. 2011). Die in dieser Arbeit nachgewiesene Relevanz einer Vermehrung von mikrozytären Megakaryozyten wurde in anderen Arbeiten zwar als nützliches diagnostisches Kriterium anerkannt, aber nicht mit der Zytogenetik korreliert.

\subsubsection{WHO-Klassifikation}

In der vorliegenden Arbeit korrelierte der IPSS-R nicht mit der WHO-Klassifikation, weder im Gesamt- noch im Score-Kollektiv. Dies scheint insofern eine überraschende Erkenntnis zu sein, als man annehmen könnte, dass ein höheres Stadium laut WHO mit einem erhöhten zytogenetischen Risiko einhergeht. Der Vergleich mit diesbezüglich publizierten Ergebnissen zeigte jedoch eine Übereinstimmung mit den Erkenntnissen aus der vorliegenden Arbeit. Im Rahmen der Umstellung der FAB-Klassifikation auf die WHO-Klassifikation kam es zu einer Aufteilung der RAEB Gruppe in RAEB-1 und RAEB-2. Dies haben einige Autoren zum Anlass genommen, die beiden Gruppen in Bezug auf die zytogenetische Zusammensetzung zu untersuchen. Breccia et al. (2009) eruierten in einer Studie mit 228 Patienten 
verschiedene prognostische Faktoren, die sich für RAEB-1 und RAEB-2 unterschieden. Bei der Betrachtung der Zytogenetik zeigte sich ein signifikant häufigeres Auftreten von Anomalien, balancierten Translokationen und komplexen Karyotypen in der RAEB-2-Gruppe. Die morphologische Diagnostik wurde anhand zytologischer Kriterien durchgeführt. Limitierend für eine Aussage über das genetische Risiko war, dass zwar die einzelnen zytogenetischen Aberration, nicht aber das assoziierte Risiko verglichen wurde. So lag bei anderen Aberrationen, wie Trisomie 8 oder Deletion 7, kein signifikanter Unterschied vor (Breccia et al. 2009).

In einer ähnlich angelegten Studie mit 558 Patienten wurde verglichen, inwiefern sich die Zytogenetik der RAEB-1- von der RAEB-2-Gruppe unterschied. Für die WHOKlassifikation wurde die zytomorphologische Beurteilung verwendet. Bei der Untersuchung hinsichtlich der Zytogenetik wurden aber sowohl die einzelnen Aberrationen als auch die Risikogruppe nach IPSS verglichen. Hierbei ergab sich für beide Einteilungen kein signifikanter Unterscheid zwischen RAEB-1 und RAEB-2. Der von Breccia et al. beobachtete Unterschied in den normalen und multiplen Aberrationen ließ sich nicht nachweisen (Germing et al. 2006a).

In einer weiteren Studie von Germing et al. mit 1095 Patienten wurden alle WHO-Subtypen im Hinblick auf ihren Einfluss auf die Prognose bei MDS untersucht. Bei der Beschreibung der zytogenetischen Zusammensetzung des Kollektivs zeigte sich ein Unterschied zwischen den WHO-Gruppen in Bezug auf den Anteil der Patienten mit nachweisbar abnormem Karyotyp (RCMD: $49 \%$ vs. RAEB-1: 61 \%, RAEB-2: $62 \%$ ). Ein leichter Unterschied zeigte sich ebenfalls zwischen RCMD, RAEB1 und RAEB-2 in Bezug auf die zytogenetische Risikoverteilung nach IPSS (0 Punkte: $70 \%$ vs. $62 \%$ vs. $60 \%$; 0,5 Punkte: $14 \%$ vs. $16 \%$ vs. 15 $\%$; 1 Punkt: $16 \%$ vs. $25 \%$ und $26 \%$ ). Eine statistische Testung dieser Daten auf Signifikanz wurde nicht durchgeführt. Für die Einteilung der morphologischen Kategorien wurde die zytologische Untersuchung gewählt (Germing et al. 2006b). Die weiterführende statistische Analyse der Daten aus dem oben genannten Düsseldorfer Kollektiv von Germing et al. erfolgte an 595 Patienten im Rahmen einer späteren Studie. Kein signifikanter Unterschied im Anteil von Hoch-Risiko-Zytogenetik zeigte sich zwischen den WHO-Gruppen RCMD vs. RCMD mit Ringsideroblasten, RAEB-1 vs. RAEB-2 sowie RCMD/RCMD mit Ringsideroblasten vs. RAEB-1/RAEB-2. Signifikant war allerdings der Unterschied zwischen RA/RARS vs. RCMD/RCMD mit Ringsideroblasten sowie vs. RAEB-1/RAEB-2 (Haase et al. 2007). 
Eine ähnliche Verteilung der Zytogenetik innerhalb der WHO-Subtypen wurde auch in anderen Veröffentlichungen beschrieben. In einer Studie mit 467 Patienten wurde ebenfalls die prognostische Aussagekraft der WHO-Klassifikation getestet. Die zytogenetischen Subgruppen nach IPSS wurden den zytologisch gestellten WHO-Diagnosen gegenübergestellt. Es zeigte sich eine Aufteilung in der zytogenetischen Subgruppe "good" von 54 \% zu 54 \% zu $52 \%$ für RCMD $(\mathrm{N}=80)$, RAEB-1 $(\mathrm{N}=59)$ und RAEB-2 $(\mathrm{N}=72)$. Für die genetische Risikogruppe "intermediate" war die Aufteilung $25 \%$ zu $32 \%$ zu $10 \%$ und für "poor" $21 \%$ zu $14 \%$ zu $38 \%$, respektive. Inwiefern diese Unterscheide signifikant waren, wurde nicht getestet. Dem Augenschein nach scheint kein eindeutiger Trend für ein erhöhtes genetisches Risiko in höheren WHO-Gruppen zu bestehen. Eine günstige Zytogenetik fand sich häufiger in der WHO-Gruppe RA ( $=76$ ) ("good": 62 \%, "intermediate": 21 \% und "poor": 17 \%) (Malcovati et al. 2005). Diese Verteilung wurde in einer aktuellen Studie mit 1150 Patienten bestätigt. Nach dem IPSS war das genetische Risiko bei RCMD zu 64 \% ,good“, zu 23\% „intermediate“ und zu $13 \%$,poor“, bei RAEB-1 analog dazu 65\%, $19 \%$ und $16 \%$ sowie bei RAEB-2 $58 \%, 28 \%$ und $14 \%$. Ob signifikante Unterschiede vorlagen, wurde nicht analysiert. Ein signifikanter Unterschied könnte sich vielleicht im Vergleich zur RCUDGruppe mit einer Aufteilung von $84 \%, 10 \%$ und 6\% ergeben (Della Porta et al. 2015).

Zusammenfassend betrachtet bestätigte der Vergleich mit der bestehenden Literatur, dass es wahrscheinlich keinen Zusammenhang zwischen dem genetischen Risiko und den WHOSubtypen MDS-MLD, MDS-EB-1 und MDS-EB-2 gibt. Nur für den günstigsten WHOSubtyp (MDS-SLD) scheint dieser zu bestehen. Wie bereits erläutert, ist dieser Subtyp einer Diagnostik durch die histologische Morphologie jedoch nur schwer zugänglich.

\subsubsection{Weitere histomorphologische Kriterien}

In der Literatur werden weitere interessante histomorphologische Kriterien im Zusammenhang mit der Zytogenetik beschrieben, die in der vorliegenden Arbeit nicht in die Bewertung einbezogen waren.

In einer Studie mit 288 Patienten wurde der Zusammenhang zwischen Eosinophilie/Basophilie und dem Gesamt-IPSS, dem zytogenetischem Risiko nach IPSS sowie dem Gesamtüberleben untersucht. Es zeigte sich, dass ein Vorkommen von $>1 \%$ Basophilen und $>5 \%$ Eonsinophilen signifikant mit einem erhöhten zytogenetischen Risiko nach IPSS und mit einem erhöhten IPSS-Gesamtscore korreliert war. In der multivariaten Analyse zeigte sich die Basophilie als unabhängiger Faktor für ein erhöhtes Risiko einer Progression zur AML (Matsushima 2003). 
Ein weiteres histomorphologisches Kriterium, das untersucht wurde, ist der Einfluss $\mathrm{CD} 271^{+}$mesenchymaler Stromazellen (MSZ). Es konnte belegt werden, dass deren Vermehrung bei höhergradigem MDS (> $5 \%$ Blasten) ein von IPSS-R, Transfusionsbedarf, t-MDS und Fibrosegrad unabhängiger Prognosefaktor für das Überleben war. Eine Korrelation mit der zytogenetischen Risikogruppe nach IPSS-R wurde nicht durchgeführt. Die Aussagekraft für die Prognose zeigte sich bei einer quantitativen Analyse des Knochenmarks (mittels automatischer Bildanalyse) und qualitativer Beurteilung des Knochenmarks (mittels Einteilung in einen von drei Schweregraden) (Johnson et al. 2014).

\subsection{Pathophysiologisches Erklärungsmodell}

Die Auswertung der histologischen Kriterien des MDS ergab, dass weder bei Reifungsstörungen (Makrozytose der Erythropoiese, Linksverschiebung der Granulopoiese, Hypolobulierung und Mikrozytose der Megakaryopoiese) oder bei Veränderungen der Architektur (ALIP der Granulopoiese, unscharfe Nester der Erythropoiese, Clusterbildung der Megakaryozyten) ein verstärktes Auftreten einer Hochrisiko-Genetik zu erwarten ist. Vielmehr waren quantitative Veränderungen der Zellreihen sowie Störungen des Markraumstromas (Fibrose, Siderose) mögliche Prädiktoren. Ob diese Veränderungen Ursache oder Resultat eines hohen genetischen Risikos waren, ließ sich aus den vorhandenen Daten nicht ableiten.

In Bezug auf erstere Annahme wurde in aktuellen Studien vielfach der Einfluss des Mikromilieus bzw. der Stammzellnische für die Entstehung von MDS diskutiert. So konnten Blau et al. (2007) bei MDS und AML in den Stromazellen zytogenetische Veränderungen nachweisen. Diese waren nicht klonal und unterschieden sich von den Aberrationen in den MDSZellen desselben Patienten. Auch Lopez-Villar et al. (2009) konnten nicht-somatische Aberrationen in 39 Patienten mit MDS nachweisen und identifizierten in den mesenchymalen Stromazellen (MSZ) eine Subgruppe von typischen Aberrationen beim 5q-Syndrom. In einer aktuellen Studie wurde diese genetische Instabilität der Stromazellen bei MDS bestätigt. In 34 \% der Fälle konnte eine Veränderung dokumentiert werden (Kouvidi et al. 2016). In einer Folgestudie konnten Blau et al. ihre Ergebnisse ebenfalls bestätigen. In 16 \% der MSZ lagen genetische Veränderungen vor (Blau et al. 2011). Interessanterweise konnte nachgewiesen werden, dass bei Patienten mit und ohne Aberration in der MSZ zwar gleichhäufig zytogenetische Veränderungen der hämatopoietischen Zelle vorlagen, diese jedoch bei Patienten mit genetischer Aberration der MSZ ausschließlich eine Hochrisiko-Zytogenetik für MDS bzw. AML darstellten. Dies legt nahe, dass eine Aberration in der MSZ mit einem erhöhten genetischen Risiko für die hämatopoietische Zelle einhergeht. Dies wiederum unterstützt die 
aus den Ergebnissen der vorliegenden Dissertation abgeleitete Hypothese eines Zusammenhangs zwischen Veränderungen des Stromas und einer Hoch-Risiko-Zytogenetik.

Abgesehen vom Nachweis genetischer Veränderungen in mesenchymalen Stromazellen konnte anhand von Mausmodellen eine Induktion der Myelodysplasie durch eine veränderte Genetik in Bestandteilen des Mikromilieus gezeigt werden. Eine Deletion des Dicer1-Gens in Mäuse-Osteoprogenitorzellen führte zu dysplastischen Veränderungen der Hämatopoiese sowie zur Entstehung einer AML, was die Autoren als Zeichen einer „Nischen-induzierten Onkogenese“ werteten (Raaijmakers et al. 2010). Ebenfalls konnte die Rolle des Retinoblastom Proteins (RB) in der Interaktion zwischen der hämatopoietischen Stammzelle und dem Stroma nachgewiesen werden, da dessen Deletion zu einer Myeloproliferation und einer extramedullären Hämatopoiese führte (Walkley et al. 2007).

Ebenfalls ein Stromafaktor, dessen Relevanz bezüglich des MDS gezeigt werden konnte, ist eine vermehrte Angiogenese (Aguayo et al. 2000), auch wenn hierfür keine prognostische Aussagekraft nachzuweisen war (Lundberg et al. 2006). Beachtenswerter Weise zeigte sich bei einer de-novo AML eine geringere Angiogenese als bei einer sekundären AML nach MDS (Keith et al. 2007).

\subsection{Ausblick}

In der vorliegenden Dissertation konnten histologische Kriterien für ein erhöhtes genetisches Risiko erarbeitet werden. Zukünftige Studien könnten die genaue Quantifizierung dieses Zusammenhangs an größeren, homogeneren Kollektiven vornehmen und die hier erarbeiteten Befunde überprüfen. Förderlich wäre der Ausschluss von Patienten mit t-MDS oder von Patienten, die sich unter Therapie befinden. Ebenfalls können die hier untersuchten Kriterien auf ihre prognostische oder differentialdiagnostische Aussagekraft hin untersucht werden. Insbesondere in Bezug auf die letztere Fragestellung ist interessant, inwiefern bestimmte Reifungs- und Architekturstörungen spezifisch für MDS sind, oder ob sie auch durch Noxen, Vitaminmangel oder andere hämato-onkologische Erkrankungen verursacht werden können. Des Weiteren sind die in der vorliegenden Arbeit gesammelten Erkenntnisse bezüglich der Betrachtung der pathophysiologischen Entstehung von MDS aus der Perspektive der hämatopoietischen Stammzellnische interessant. Ungeklärt ist weiterhin, ob strukturelle Veränderungen des Stromas Ursache oder Auswirkung der myelodysplastischen Veränderungen des Knochenmarks sind. 


\section{$5 \quad$ Zusammenfassung}

Beim myelodysplastischen Syndrom handelt es sich um eine komplexe heterogene Erkrankung. Sowohl das Stellen der Diagnose als auch die Bestimmung der Prognose ist in vielen Fällen eine Herausforderung. Die vorliegende Dissertation hat anhand der Befunde und Daten von 91 Patienten mit MDS untersucht, inwiefern sich die WHO-Klassifikation auf Basis der Knochenmarkshistologie reproduzieren lässt und welchen prädiktiven Wert histopathologische Kriterien für das zytogenetische Risiko bei MDS besitzen.

Zuerst wurden die in der normalen pathologischen Diagnostik erhobenen histologischen Kriterien evaluiert. In einem zweiten Schritt wurde ein Subkollektiv mit Patienten gebildet, die mit hoher diagnostischer Sicherheit an einem MDS erkrankt waren (erhöhter Blastenanteil, positive Zytogenetik). Die verschiedenen in den Gewebsproben dieser Patienten $(\mathrm{N}=$ 55) zu erkennenden Reifungs- und Architekturstörungen wurden anhand einer semi-quantitativen Skala bewertet. Die WHO-Kategorien MDS-MLD, MDS-EB-1 sowie MDS-EB-2 konnten histologisch abgebildet werden. Histologische Kriterien wurden ermittelt, die mit einem erhöhten zytogenetischen Risiko laut IPSS-R einhergingen. Hierzu gehörten die Markraumfibrose, eine starke Hyperzellularität sowie eine Vermehrung von Megakaryozyten, während der Einfluss einer Siderose des Markraums nicht eindeutig nachgewiesen werden konnte. Keinen Einfluss hatten bestimmte zelluläre Veränderungen (Makrozytose und Kerngrößenvariabilität der Erythropoiese, Hypolobulierung, Mikrozytose und CD34-Expression der Megakaryopoiese, Linksverschiebung und Größenvariabilität der Granulopoiese) oder Architekturstörungen im Knochenmark (ALIP der Granulopoiese, unscharfe Nester der Erythropoiese, Clusterbildung der Megakaryopoiese). Auch ein höheres Stadium laut WHOKlassifikation ging nicht mit einem gesteigerten zytogenetischen Risiko einher. Eine vermehrte Granulopoiese könnte als Indiz für eine bessere Prognose nach IPSS-R gewertet werden.

Die vorliegende Dissertation liefert einen Beitrag dazu, die Rolle der histopathologischen Diagnostik im Zusammenspiel den verschiedenen Untersuchungsverfahren bei MDS zu verorten. Ebenfalls ermöglicht sie, relevante histologische Kriterien für ein erhöhtes zytogenetisches Risiko von irrelevanten zu unterscheiden. Zukünftige Studien sollten versuchen, den Einfluss dieser Parameter an einem homogeneren, größeren Kollektiv genauer zu quantifizieren. Ebenfalls können die hier erarbeiteten Kriterien für prognostische oder (differential)diagnostische Fragestellungen genutzt werden. 


\section{Literaturverzeichnis}

Aanei CM, Flandrin P, Eloae FZ, Carasevici E, Guyotat D, Wattel E, Campos L (2012): Intrinsic Growth Deficiencies of Mesenchymal Stromal Cells in Myelodysplastic Syndromes. Stem Cells Dev 21, 1604-1615

Adès L, Itzykson R, Fenaux P (2014): Myelodysplastic syndromes. Lancet $\underline{383}$, 2239-2252 Afable MG, Tiu RV, Maciejewski JP (2011): Clonal Evolution in Aplastic Anemia. Hematology Am Soc Hematol Educ Program 2011, 90-95

Aguayo A, Kantarjian H, Manshouri T, Gidel C, Estey E, Thomas D, Koller C, Estrov Z, O'Brien S, Keating M et al. (2000): Angiogenesis in acute and chronic leukemias and myelodysplastic syndromes. Blood 므, 2240-2245

Arber DA, Orazi A, Hasserjian R, Thiele J, Borowitz MJ, Le Beau MM, Bloomfield CD, Cazzola M, Vardiman JW (2016): The 2016 revision to the World Health Organization classification of myeloid neoplasms and acute leukemia. Blood 127, 2391-2405

Bacher U, Schanz J, Braulke F, Haase D (2014): Rare cytogenetic abnormalities in myelodysplastic syndromes. Mediterr J Hematol Infect Dis $\underline{7}$, e2015034

Bain BJ (2001): Bone marrow trephine biopsy. J Clin Pathol 54, 737-742

Bain BJ (2005): Diagnosis from the blood smear. N Engl J Med $\underline{353}$, 498-507

Bain BJ, Clark DM, Wilkins BS: Bone marrow pathology. 4. Auflage; Wiley-Blackwell, Oxford, 2010

Barlow JL, Drynan LF, Hewett DR, Holmes LR, Lorenzo-Abalde S, Lane AL, Jolin HE, Pannell R, Middleton AJ, Wong SH et al. (2010): A p53-dependent mechanism underlies macrocytic anemia in a mouse model of human 5q- syndrome. Nat Med 16, 59-66

Baur AS, Meuge-Moraw C, Schmidt P, Parlier V, Jotterand M, Delacretaz F (2000): CD34/QBEND10 immunostaining in bone marrow biopsies: An additional parameterfor the diagnosis and classification of myelodysplastic syndromes. Eur J Haematol $\underline{64}, 71-79$

Bejar R, Steensma DP (2014): Recent developments in myelodysplastic syndromes. Blood $\underline{124}, 2793-2803$

Bejar R, Stevenson K, Abdel-Wahab O (2011): Clinical effect of point mutations in myelodysplastic syndromes. N Engl J Med 364, 2496-2506 
Bennett JM, Catovsky D, Daniel M, Flandrin G, Galton DAG, Gralnick HR, Sultan C (1976): Proposals for the Classification of the Acute Leukaemias French-American-British (FAB) Co-operative Group. Br J Haematol $\underline{33}$, 451-458

Bennett JM, Catovsky D, Daniel MT, Flandrin G, Galton DA, Gralnick HR, Sultan C (1982): Proposals for the classification of the myelodysplastic syndromes. Br J Haematol 51, 189-199

Björk J, Albin M, Mauritzson N, Strömberg U, Johansson B, Hagmar L (2000): Smoking and myelodysplastic syndromes. Epidemiology 11, 285-291

Blau O, Hofmann W, Baldus CD, Thiel G, Serbent V, Schümann E, Thiel E, Blau IW (2007): Chromosomal aberrations in bone marrow mesenchymal stroma cells from patients with myelodysplastic syndrome and acute myeloblastic leukemia. Exp Hematol $\underline{35}$, 221229

Blau O, Baldus CD, Hofmann WK, Thiel G, Nolte F, Burmeister T, Turkmen S, Benlasfer O, Schumann E, Sindram A et al. (2011): Mesenchymal stromal cells of myelodysplastic syndrome and acute myeloid leukemia patients have distinct genetic abnormalities compared with leukemic blasts. Blood 118, 5583-5592

Block M, Jacobson L, Bethard W (1953): Preleukemic acute human leukemia. J Am Med Assoc 152, 1018-1028

Braulke F, Schanz J, Jung K, Shirneshan K, Schulte K, Schuetze C, Steffens R, Trümper L, Haase D (2010): FISH analysis of circulating CD34+ cells as a new tool for genetic monitoring in MDS: verification of the method and application to 27 MDS patients. Leuk Res $\underline{34}, 1296-1301$

Braulke F, Jung K, Schanz J, Götze K, Müller-Thomas C, Platzbecker U, Germing U, Brümmendorf TH, Bug G, Ottmann O et al. (2013): Molecular cytogenetic monitoring from CD34+ peripheral blood cells in myelodysplastic syndromes: first results from a prospective multicenter German diagnostic study. Leuk Res 37, 900-906 Braulke F, Platzbecker U, Müller-Thomas C, Götze K, Germing U, Brümmendorf TH, Nolte F, Hofmann WK, Giagounidis AA, Lübbert M et al. (2015): Validation of cytogenetic risk groups according to International Prognostic Scoring Systems by peripheral blood CD34+FISH: results from a German diagnostic study in comparison with an international control group. Haematologica 100, 205-213 
Breccia M, Latagliata R, Cannella L, Carmosino I, Cuia R de, Frustaci A, Stefanizzi C, Santopietro M, Alimena G (2009): Analysis of prognostic factors in patients with refractory anemia with excess of blasts (RAEB) reclassified according to WHO proposal. Leuk Res $\underline{33}, 391-394$

Bryan J, Jabbour E, Prescott H, Kantarjian H (2010): Thrombocytopenia in patients with myelodysplastic syndromes. Semin Hematol 47, 274-280

Buckley SM, Verfaillie C: Regulation of hematopoiesis. In: Porwit A, McCullough J, Erber WN (Hrsg.): Blood and bone marrow pathology. 2. Auflage; Churchill Livingstone Elsevier, Edinburgh u. a. 2011, 63-78

Buesche G, Teoman H, Wilczak W, Ganser A, Hecker H, Wilkens L, Göhring G, Schlegelberger B, Bock O, Georgii A et al. (2008): Marrow fibrosis predicts early fatal marrow failure in patients with myelodysplastic syndromes. Leukemia 22, 313-322

Chai X, Li D, Cao X, Zhang Y, Mu J, Lu W, Xiao X, Li C, Meng J, Chen J et al. (2015): ROS-mediated iron overload injures the hematopoiesis of bone marrow by damaging hematopoietic stem/progenitor cells in mice. Sci Rep $\underline{5}, 10181$

Chasis JA, Mohandas N (2008): Erythroblastic islands: niches for erythropoiesis. Blood $\underline{112}, 470-478$

Churpek JE, Larson RA (2013): The evolving challenge of therapy-related myeloid neoplasms. Best Pract Res Clin Haematol 26, 309-317

Cid J, Palomera L, Diaz M, Zamora C, Solano F (2014): Clinical characteristics and management of iron overload in 631 patients with chronic transfusion dependency: results from a multicentre, observational study. Blood Transf 12 Suppl 1, s119-23

Civin CI, Strauss LC, Brovall C, Fackler MJ, Schwartz JF, Shaper JH (1984): Antigenic analysis of hematopoiesis. III. A hematopoietic progenitor cell surface antigen defined by a monoclonal antibody raised against KG-1a cells. J Immunol 133, 157-165

Cogle CR, Craig BM, Rollison DE, List AF (2011): Incidence of the myelodysplastic syndromes using a novel claims-based algorithm: high number of uncaptured cases by cancer registries. Blood $\underline{117}, 7121-7125$

Corey SJ, Minden MD, Barber DL, Kantarjian H, Wang JCY, Schimmer AD (2007): Myelodysplastic syndromes: the complexity of stem-cell diseases. Nat Rev Cancer $\underline{7}, 118-129$ 
Das R, Hayer J, Dey P, Garewal G (2005): Comparative study of myelodysplastic syndromes and normal bone marrow biopsies with conventional staining and immunocytochemistry. Anal Quant Cytol Histol 27, 152-156

De Roos AJ, Deeg HJ, Onstad L, Kopecky KJ, Bowles EJA, Yong M, Fryzek J, Davis S (2010): Incidence of myelodysplastic syndromes within a nonprofit healthcare system in western Washington state, 2005-2006. Am J Hematol 85, 765-770

Della Porta MG, Malcovati L, Boveri E, Travaglino E, Pietra D, Pascutto C, Passamonti F, Invernizzi R, Castello A, Magrini U et al. (2009): Clinical relevance of bone marrow fibrosis and CD34-positive cell clusters in primary myelodysplastic syndromes. J Clin Oncol 27, $754-762$

Della Porta MG, Travaglino E, Boveri E, Ponzoni M, Malcovati L, Papaemmanuil E, Rigolin GM, Pascutto C, Croci G, Gianelli U et al. (2015): Minimal morphological criteria for defining bone marrow dysplasia: a basis for clinical implementation of WHO classification of myelodysplastic syndromes. Leukemia $\underline{29}$, 66-75

Deutsch VR, Tomer A (2006): Megakaryocyte development and platelet production. Br J Haematol 134, 453-466

Dick F, Tevaearai H (2015): Significance and Limitations of the p Value. Eur J Vasc Endo$\operatorname{vasc} \operatorname{Surg} \underline{50}, 815$

Du Prel JB, Röhrig B, Hommel G, Blettner M (2010): Choosing Statistical Tests_-Part 12 of a Series on Evaluation of Scientific Publikations. Dtsch Arztebl Int 107, 343-348

Ebert BL, Pretz J, Bosco J, Chang CY, Tamayo P, Galili N, Raza A, Root DE, Attar E, Ellis SR et al. (2008): Identification of RPS14 as a 5q- syndrome gene by RNA interference screen. Nature $451,335-339$

Faller H (2004): Signifikanz, Effektstärke und Konfidenzintervall. Rehabilitation $\underline{43}, 174$ 178

Faulkner-Jones BE, Howie AJ, Boughton BJ, Franklin IM (1988): Lymphoid aggregates in bone marrow: study of eventual outcome. J Clin Pathol $\underline{41}, 768-775$

Font P, Loscertales J, Benavente C, Bermejo A, Callejas M, Garcia-Alonso L, GarciaMarcilla A, Gil S, Lopez-Rubio M, Martin E et al. (2013): Inter-observer variance with the diagnosis of myelodysplastic syndromes (MDS) following the 2008 WHO classification. Ann Hematol 92, 19-24 
Font P, Loscertales J, Soto C, Ricard P, Novas CM, Martín-Clavero E, López-Rubio M, Garcia-Alonso L, Callejas M, Bermejo A et al. (2015): Interobserver variance in myelodysplastic syndromes with less than $5 \%$ bone marrow blasts: Unilineage vs. multilineage dysplasia and reproducibility of the threshold of $2 \%$ blasts. Ann Hematol 94, 565-573

Foran JM, Shammo JM (2012): Clinical Presentation, Diagnosis, and Prognosis of Myelodysplastic Syndromes. Am J Med 125, S6-S13

Fu B, Ok CY, Goswami M, Xei W, Jaso JM, Muzzafar T, Bueso-Ramos C, Verstovsek S, Garcia-Manero G, Medeiros LJ et al. (2013): The clinical importance of moderate/severe bone marrow fibrosis in patients with therapy-related myelodysplastic syndromes. Ann Hematol $\underline{\text { 22, }}$ 1335-1343

Fu B, Jaso JM, Sargent RL, Goswami M, Verstovsek S, Medeiros LJ, Wang SA (2014):

Bone marrow fibrosis in patients with primary myelodysplastic syndromes has prognostic value using current therapies and new risk stratification systems. Mod Pathol 27, 681-689

Garcia-Manero G (2014): Myelodysplastic syndromes: 2014 update on diagnosis, risk-stratification, and management. Am J Hematol $\underline{89}$, 97-108

Gaus W, Muche R: Medizinische Statistik: Angewandte Biometrie für Ärzte und Gesundheitsberufe. Schattauer, Stuttgart 2014

Germing U, Strupp C, Kuendgen A, Aivado M, Giagounidis A, Hildebrandt B, Aul C, Haas R, Gattermann N (2006a): Refractory anaemia with excess of blasts (RAEB): analysis of reclassification according to the WHO proposals. Br J Haematol 132, 162-167

Germing U, Strupp C, Kuendgen A, Isa S, Knipp S, Hildebrandt B, Giagounidis A, Aul C, Gattermann N, Haas R (2006b): Prospective validation of the WHO proposals for the classification of myelodysplastic syndromes. Haematologica 91, 1596-1604

Germing U, Strupp C, Giagounidis A, Haas R, Gattermann N, Starke C, Aul C (2012): Evaluation of dysplasia through detailed cytomorphology in 3156 patients from the Düsseldorf Registry on myelodysplastic syndromes. Leuk Res $\underline{36}$, 727-734

Goasguen JE, Bennett JM, Bain BJ, Brunning R, Vallespi M, Tomonaga M, Zini G, Renault A (2014): Proposal for refining the definition of dysgranulopoiesis in acute myeloid leukemia and myelodysplastic syndromes. Leuk Res $\underline{38}, 447-453$

Godley LA, Larson RA (2008): Therapy-related myeloid leukemia. Semin Oncol $\underline{35}$, 418429 
Goncalves AC, Cortesao E, Oliveiros B, Alves V, Espadana AI, Rito L, Magalhaes E, Lobao MJ, Pereira A, Nascimento Costa JM et al. (2015): Oxidative stress and mitochondrial dysfunction play a role in myelodysplastic syndrome development, diagnosis, and prognosis: A pilot study. Free Radic Res $\underline{49}$, 1081-1094

Greenberg P, Cox C, LeBeau MM, Fenaux P, Morel P, Sanz G, Sanz M, Vallespi T, Hamblin T, Oscier D (1997): International scoring system for evaluating prognosis in myelodys-

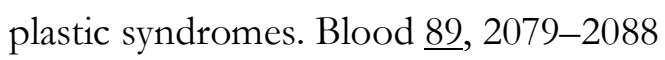

Greenberg PL, Tuechler H, Schanz J, Sanz G, Garcia-Manero G, Sole F, Bennett JM, Bowen D, Fenaux P, Dreyfus F et al. (2012): Revised International Prognostic Scoring System for Myelodysplastic Syndromes. Blood 120, 2454-2465

Guyotat D, Campos L, Thomas X, Vila L, Shi ZH, Charrin C, Gentilhomme O, Fiere D (1990): Myelodysplastic syndromes: a study of surface markers and in vitro growth patterns. Am J Hematol $\underline{34}$, 26-31

Haase D, Germing U, Schanz J, Pfeilstöcker M, Nösslinger T, Hildebrandt B, Kundgen A, Lübbert M, Kunzmann R, Giagounidis AAN (2007): New insights into the prognostic impact of the karyotype in MDS and correlation with subtypes: evidence from a core dataset of 2124 patients. Blood 110, 4385-4395

Haferlach C, Rieder H, Lillington DM, Dastugue N, Hagemeijer A, Harbott J, Stilgenbauer S, Knuutila S, Johansson B, Fonatsch C (2007): Proposals for standardized protocols for cytogenetic analyses of acute leukemias, chronic lymphocytic leukemia, chronic myeloid leukemia, chronic myeloproliferative disorders, and myelodysplastic syndromes. Genes Chromosomes Cancer 46, 494-499

Haferlach T, Nagata Y, Grossmann V, Okuno Y, Bacher U, Nagae G, Schnittger S, Sanada M, Kon A, Alpermann T et al. (2014): Landscape of genetic lesions in 944 patients with myelodysplastic syndromes. Leukemia 28 , 241-247

Hanspal M, Smockova Y, Uong Q (1998): Molecular identification and functional characterization of a novel protein that mediates the attachment of erythroblasts to macrophages. Blood 92, 2940-2950

Hartmann J, Braulke F, Sinzig U, Wulf G, Maas JH, Konietschke F, Haase D (2013): Iron overload impairs proliferation of erythroid progenitors cells (BFU-E) from patients with myelodysplastic syndromes. Leuk Res $\underline{37}, 327-332$ 
Holme H, Hossain U, Kirwan M, Walne A, Vulliamy T, Dokal I (2012): Marked genetic heterogeneity in familial myelodysplasia/acute myeloid leukaemia. Br J Haematol 158, 242 248

Horny H, Sotlar K, Valent P (2007): Diagnostic value of histology and immunohistochemistry in myelodysplastic syndromes. Leuk Res $\underline{31}, 1609-1616$

Horny HP, Wehrmann M, Schlicker HU, Eichstaedt A, Clemens MR, Kaiserling E (1995): QBEND10 for the diagnosis of myelodysplastic syndromes in routinely processed bone marrow biopsy specimens. J Clin Pathol 4ㄹ, 291-294

Howe RB (2004): The WHO classification of MDS does make a difference. Blood 103 , $3265-3270$

Insuasti-Beltran G, Steidler NL, Kang H, Reichard KK (2012): CD34+ megakaryocytes $(\geq 30 \%)$ are associated with megaloblastic anaemia and non-acute myeloid neoplasia. Histopathology $\underline{61,694-701}$

Ishiyama K, Chuhjo T, Wang H, Yachie A, Omine M (2003): Polyclonal hematopoiesis maintained in patients with bone marrow failure harboring a minor population of paroxysmal nocturnal hemoglobinuria-type cells. Blood 102, 1211-1216

Jaffe ES, Harris NL, Stein H, Vardiman JW (Hrg.): Pathology and genetics of tumours of haematopoietic and lymphoid tissues (World Health Organization classification of tumours). Band 2. 3. Auflage; International Agency for Research on Cancer, Genf 2001 Jaiswal S, Fontanillas P, Flannick J, Manning A, Grauman PV, Mar BG, Lindsley RC, Mermel CH, Burtt N, Chavez A et al. (2014): Age-related clonal hematopoiesis associated with adverse outcomes. N Engl J Med 371, 2488-2498

Jiang Y, Dunbar A, Gondek LP, Mohan S, Rataul M, O'Keefe C, Sekeres M, Saunthararajah Y, Maciejewski JP (2009): Aberrant DNA methylation is a dominant mechanism in MDS progression to AML. Blood 113, 1315-1325

Jin J, Yu M, Hu C, Ye L, Xie L, Jin J, Chen F, Tong H (2014): Pesticide exposure as a risk factor for myelodysplastic syndromes: a meta-analysis based on 1,942 cases and 5,359 controls. PloS One $\underline{9}$, e110850

Johnson RC, Kurzer JH, Greenberg PL, Gratzinger D (2014): Mesenchymal stromal cell density is increased in higher grade myelodysplastic syndromes and independently predicts survival. Am J Clin Pathol 142, 795-802 
Kantarjian HM, Keating MJ, Walters RS, Smith TL, Cork A, McCredie KB, Freireich EJ (1986): Therapy-related leukemia and myelodysplastic syndrome: clinical, cytogenetic, and prognostic features. J Clin Oncol 4, 1748-1757

Kanter-Lewensohn L, Hellström-Lindberg E, Kock Y, Elmhorn-Rosenborg A, Ost A (1996): Analysis of CD34-positive cells in bone marrow from patients with myelodysplastic syndromes and acute myeloid leukemia and in normal individuals: A comparison between FACS analysis and immunohistochemistry. Eur J Haematol 56, 124-129

Kawai N, Matsuda A, Jinnai I, Ichimura T, Kayano H, Okamura D, Ishikawa M, Maeda T, Hata T, Miyazaki Y et al. (2016): Proposal of criteria for dyserythropoiesis in the diagnosis of myelodysplastic syndromes. Int J Hematol $\underline{103}, 227-233$

Keith T, Araki Y, Ohyagi M, Hasegawa M, Yamamoto K, Kurata M, Nakagawa Y, Suzuki K, Kitagawa M (2007): Regulation of angiogenesis in the bone marrow of myelodysplastic syndromes transforming to overt leukaemia. Br J Haematol 137, 206-215

Kitagawa M, Kamiyama R, Takemura T, Kasuga T (1989): Bone marrow analysis of the myelodysplastic syndromes: histological and immunohistochemical features related to the evolution of overt leukemia. Virchows Arch B Cell Pathol Incl Mol Pathol 57, 47-53

Kobayashi Y, Kimura S, Tanaka K, Wada K, Ozawa M, Horiuchi H, Maruo N, Kondo M (1991): Shift in the megakaryocyte ploidy in MDS patients: microcytofluorometry with DAPI staining after destaining of Wright-Giemsa stain. Br J Haematol 79, 556-561

Kobayashi Y, Uoshima N, Kimura S, Tanaka K, Wada K, Ozawa M, Maruo N, Kondo M (1995): Relationship between morphological classification of the degree of maturation and the ploidy of micromegakaryocytes in myelodysplastic syndrome patients. Int J Hematol 61, 117-122

Kodali D, Mesa H, Rawal A, Cao Q, Gupta P (2007): Thrombocytosis in myelodysplastic and myelodysplastic/myeloproliferative syndromes. Leuk Lymphoma 48, 2375-2380

Kordasti SY, Ingram W, Hayden J, Darling D, Barber L, Afzali B, Lombardi G, Wlodarski MW, Maciejewski JP, Farzaneh F et al. (2007): CD4+CD25high Foxp3+ regulatory T cells in myelodysplastic syndrome (MDS). Blood 110, 847-850

Kotsianidis I, Bouchliou I, Nakou E, Spanoudakis E, Margaritis D, Christophoridou AV, Anastasiades A, Tsigalou C, Bourikas G, Karadimitris A et al. (2009): Kinetics, function and bone marrow trafficking of CD4+CD25+FOXP3+ regulatory T cells in myelodysplastic syndromes (MDS). Leukemia 23 , 510-518 
Kouvidi E, Stratigi A, Batsali A, Mavroudi I, Mastrodemou S, Ximeri M, Papadaki HA, Pontikoglou CG (2016): Cytogenetic evaluation of mesenchymal stem/stromal cells from patients with myelodysplastic syndromes at different time-points during ex vivo expansion. Leuk Res $\underline{43}, 24-32$

Krause DS, Fackler MJ, Civin CI, May WS (1996): CD34: structure, biology, and clinical utility. Blood $\underline{87}, 1-13$

Kutler DI, Singh B, Satagopan J, Batish SD, Berwick M, Giampietro PF, Hanenberg H, Auerbach AD (2003): A 20-year perspective on the International Fanconi Anemia Registry (IFAR). Blood 101, 1249-1256

Lambertenghi-Deliliers G, Orazi A, Luksch R, Annaloro C, Soligo D (1991): Myelodysplastic syndrome with increased marrow fibrosis: a distinct clinico-pathological entity. $\mathrm{Br} \mathrm{J}$ Haematol $\underline{78}, 161-166$

Lambertenghi-Deliliers G, Annaloro C, Oriani A, Soligo D, Pozzoli E, Polli EE (1993): Prognostic relevance of histological findings on bone marrow biopsy in myelodysplastic syndromes. Ann Hematol 66, 85-91

Lambertenghi-Deliliers G, Annaloro C, Soligo DV, Oriani A (1998): The diagnostic and prognostic value of bone marrow immunostaining in myelodysplastic syndromes. Leuk Lymphoma $\underline{28}, 231-239$

Lee G, Lo A, Short SA, Mankelow TJ, Spring F, Parsons SF, Yazdanbakhsh K, Mohandas N, Anstee DJ, Chasis JA (2006): Targeted gene deletion demonstrates that the cell adhesion molecule ICAM-4 is critical for erythroblastic island formation. Blood 108, 2064-2071

Leitch HA (2007): Improving clinical outcome in patients with myelodysplastic syndrome and iron overload using iron chelation therapy. Leuk Res 31 Suppl 3, S7-9

Li X, Pu Q (2005): Megakaryocytopoiesis and apoptosis in patients with myelodysplastic syndromes. Leuk Lymphoma 46, 387-391

Lopez-Villar O, Garcia JL, Sanchez-Guijo FM, Robledo C, Villaron EM, HernandezCampo P, Lopez-Holgado N, Diez-Campelo M, Barbado MV, Perez-Simon JA et al. (2009): Both expanded and uncultured mesenchymal stem cells from MDS patients are genomically abnormal, showing a specific genetic profile for the $5 \mathrm{q}-$ syndrome. Leukemia $2 \underline{3}$, 664-672 
Lundberg LG, Hellström-Lindberg E, Kanter-Lewensohn L, Lerner R, Palmblad J (2006): Angiogenesis in relation to clinical stage, apoptosis and prognostic score in myelodysplastic syndromes. Leuk Res $\underline{30}$, 247-253

Lyons RM (2012): Myelodysplastic syndromes: therapy and outlook. Am J Med 125, S18S23

Ma X (2012): Epidemiology of myelodysplastic syndromes. Am J Med 125, S2-S5

Ma X, Does M, Raza A, Mayne ST (2007): Myelodysplastic syndromes Incidence and Survival in the United States. Cancer 109, 1536-1542

Ma X, Lim U, Park Y, Mayne ST, Wang R, Hartge P, Hollenbeck AR, Schatzkin A (2009): Obesity, lifestyle factors, and risk of myelodysplastic syndromes in a large US cohort. Am J Epidemiol 169, 1492-1499

Magalhães SMM, Filho FDR, Vassallo J, Pinheiro MP, Metze K, Lorand-Metze I (2002): Bone marrow lymphoid aggregates in myelodysplastic syndromes: incidence, immunomorphological characteristics and correlation with clinical features and survival. Leuk Res $\underline{26}$, $525-530$

Malcovati L, Della Porta MG, Pascutto C, Invernizzi R, Boni M, Travaglino E, Passamonti F, Arcaini L, Maffioli M, Bernasconi P et al. (2005): Prognostic factors and life expectancy in myelodysplastic syndromes classified according to WHO criteria: a basis for clinical decision making. J Clin Oncol 23, 7594-7603

Malcovati L, Germing U, Kuendgen A, Della Porta MG, Pascutto C, Invernizzi R, Giagounidis A, Hildebrandt B, Bernasconi P, Knipp S et al. (2007): Time-dependent prognostic scoring system for predicting survival and leukemic evolution in myelodysplastic syndromes. J Clin Oncol 25, 3503-3510

Mangi M, Mufti G (1992): Primary myelodysplastic syndromes: diagnostic and prognostic significance of immunohistochemical assessment of bone marrow biopsies. Blood $\underline{79}$, 198205

Mangi MH, Salisbury JR, Mufti GJ (1991): Abnormal localization of immature precursors (ALIP) in the bone marrow of myelodysplastic syndromes: current state of knowledge and future directions. Leuk Res $\underline{15}$, 627-639

Matsushima T (2003): Prevalence and clinical characteristics of myelodysplastic syndrome with bone marrow eosinophilia or basophilia. Blood 101, 3386-3390 
Mauritzson N, Albin M, Rylander L, Billström R, Ahlgren T, Mikoczy Z, Björk J, Strömberg U, Nilsson PG, Mitelman F et al. (2002): Pooled analysis of clinical and cytogenetic features in treatment-related and de novo adult acute myeloid leukemia and myelodysplastic syndromes based on a consecutive series of 761 patients analyzed 1976-1993 and on 5098 unselected cases reported in the literature 1974-2001. Leukemia 16, 2366-2378 McQuilten ZK, Wood EM, Polizzotto MN, Campbell LJ, Wall M, Curtis DJ, Farrugia H, McNeil JJ, Sundararajan V (2014): Underestimation of myelodysplastic syndrome incidence by cancer registries: Results from a population-based data linkage study. Cancer 120, 16861694

Merlat A, Lai JL, Sterkers Y, Demory JL, Bauters F, Preudhomme C, Fenaux P (1999):

Therapy-related myelodysplastic syndrome and acute myeloid leukemia with 17p deletion. A report on 25 cases. Leukemia $\underline{13}, 250-257$

Mian SA, Smith AE, Kulasekararaj AG, Kizilors A, Mohamedali AM, Lea NC, Mitsopoulos K, Ford K, Nasser E, Seidl T et al. (2013): Spliceosome mutations exhibit specific associations with epigenetic modifiers and proto-oncogenes mutated in myelodysplastic syndrome. Haematologica $\underline{98}, 1058-1066$

Mohandas N, Chasis JA (2010): The erythroid niche: molecular processes occurring within erythroblastic islands. Transfus Clin Biol $\underline{17}, 110-111$

Moonim MT, Porwit A: Normal bone marrow histology. In: Porwit A, McCullough J, Erber WN (Hrsg.): Blood and bone marrow pathology. 2. Auflage; Churchill Livingstone Elsevier, Edinburgh u. a. 2011, 45-62

Muche R (2008): Die logistische Regression - ein vielseitiges Analyseinstrument rehabilitationswissenschaftlicher Forschung. Rehabilitation 47, 56-62

Mufti GJ, Bennett JM, Goasguen J, Bain BJ, Baumann I, Brunning R, Cazzola M, Fenaux P, Germing U, Hellström-Lindberg E et al. (2008): Diagnosis and classification of myelodysplastic syndrome: International Working Group on Morphology of myelodysplastic syndrome (IWGM-MDS) consensus proposals for the definition and enumeration of myeloblasts and ring sideroblasts. Haematologica $\underline{93}, 1712-1717$

Mulisch M, Welsch U (Hrsg.): Romeis Mikroskopische Technik, 18. Auflage; Spektrum Akademischer Verlag, Heidelberg 2010

Naqvi K, Jabbour E, Bueso-Ramos C, Pierce S, Borthakur G, Estrov Z, Ravandi F, Faderl S, Kantarjian H, Garcia-Manero G (2011): Implications of discrepancy in morphologic 
diagnosis of myelodysplastic syndrome between referral and tertiary care centers. Blood $\underline{118}, 4690-4693$

Neukirchen J, Schoonen WM, Strupp C, Gattermann N, Aul C, Haas R, Germing U (2011): Incidence and prevalence of myelodysplastic syndromes: data from the Düsseldorf MDS-registry. Leuk Res 35, 1591-1596

Niemeyer CM, Baumann I (2008): Myelodysplastic syndrome in children and adolescents. Semin Hematol 45, 60-70

Nimer SD (2008): Myelodysplastic syndromes. Blood 111, 4841-4851

Oertel J, Huhn D (1994): CD34 Immunophenotyping of blasts in myelodysplasia. Leuk Lymphoma 15, 65-69

Oertel J, Kleiner S, Huhn D (1993): Immunotyping of blasts in refractory anaemia with excess of blasts. Br J Haematol $\underline{84}$, 305-309

Oertel J, Oertel B, Beyer J, Huhn D (1994): CD 34 immunotyping of blasts in myelodysplasia. Ann Hematol $\underline{68}, 77-80$

Ohshima K, Kikuchi M, Takeshita M (1995): A megakaryocyte analysis of the bone marrow in patients with myelodysplastic syndrome, myeloproliferative disorder and allied disorders. J Pathol 177, 181-189

Okabe H, Suzuki T, Uehara E, Ueda M, Nagai T, Ozawa K (2014): The bone marrow hematopoietic microenvironment is impaired in iron-overloaded mice. Eur J Haematol $\underline{93}, 118$ 128

Olney HJ, Mitelman F, Johansson B, Mrózek K, Berger R, Rowley JD (2002): Unique balanced chromosome abnormalities in treatment-related myelodysplastic syndromes and acute myeloid leukemia: report from an international workshop. Genes Chromosomes Cancer $\underline{33}, 413-423$

Orazi A, Cattoretti G, Soligo D, Luksch R, Lambertenghi-Deliliers G (1993): Therapy-related myelodysplastic syndromes: FAB classification, bone marrow histology, and immunohistology in the prognostic assessment. Leukemia $\underline{7}$, 838-847

Oriani A, Annaloro C, Soligo D, Pozzoli E, Cortelezzi A, Lambertenghi Deliliers G (1996): Bone marrow histology and CD34 immunostaining in the prognostic evaluation of primary myelodysplastic syndromes. Br J Haematol 92, 360-364

Park S (2016): Significant results: Statistical or clinical? Korean J Anesthesiol 69, 121-125 
Passweg JR, Giagounidis AA, Simcock M, Aul C, Dobbelstein C, Stadler M, Ossenkoppele G, Hofmann WK, Schilling K, Tichelli A et al. (2011): Immunosuppressive therapy for patients with myelodysplastic syndrome: a prospective randomized multicenter phase III trial comparing antithymocyte globulin plus cyclosporine with best supportive care-SAKK 33/99. J Clin Oncol 29, 303-309

Pedersen-Bjergaard J, Philip P, Larsen SO, Andersson M, Daugaard G, Ersbøll J, Hansen SW, Hou-Jensen K, Nielsen D, Sigsgaard TC (1993): Therapy-related myelodysplasia and acute myeloid leukemia. Cytogenetic characteristics of 115 consecutive cases and risk in seven cohorts of patients treated intensively for malignant diseases in the Copenhagen series. Leukemia 7, 1975-1986

Pellagatti A (2006): Gene expression profiles of CD34+ cells in myelodysplastic syndromes: Involvement of interferon-stimulated genes and correlation to FAB subtype and karyotype. Blood 108, 337-345

Pellagatti A, Marafioti T, Paterson JC, Malcovati L, Della Porta MG, Jädersten M, Pushkaran B, George TI, Arber DA, Killick S et al. (2009): Marked downregulation of the granulopoiesis regulator LEF1 is associated with disease progression in the myelodysplastic syndromes. Br J Haematol 146, 86-90

Pellegrini W, Facchetti F, Marocolo D, Pelizzari AM, Capucci A, Rossi G (2000): Expression of CD34 by megakaryocytes in myelodysplastic syndromes. Haematologica $\underline{85}, 1117-$ 1118

Porwit A, McCullough J, Erber WN: Blood and bone marrow pathology: Expert consult, 2nd ed; Churchill Livingstone, S.1. 2011

Pozdnyakova O, Miron PM, Tang G, Walter O, Raza A, Woda B, Wang SA (2008): Cytogenetic abnormalities in a series of 1029 patients with primary myelodysplastic syndromes. Cancer 113, 3331-3340

Raaijmakers MHGP, Mukherjee S, Guo S, Zhang S, Kobayashi T, Schoonmaker JA, Ebert BL, Al-Shahrour F, Hasserjian RP, Scadden EO et al. (2010): Bone progenitor dysfunction induces myelodysplasia and secondary leukaemia. Nature $\underline{464}, 852-857$

Ramos F, Robledo C, Izquierdo-Garcia FM, Suarez-Vilela D, Benito R, Fuertes M, Insunza A, Barragan E, Del RM, Morales JM de et al. (2016): Bone marrow fibrosis in myelodysplastic syndromes: a prospective evaluation including mutational analysis. Oncotarget, $\mathbf{7}$, 30492-30503 
Raza A, Gezer S, Mundle S, Gao XZ, Alvi S, Borok R, Rifkin S, Iftikhar A, Shetty V, Parcharidou A (1995): Apoptosis in bone marrow biopsy samples involving stromal and hematopoietic cells in 50 patients with myelodysplastic syndromes. Blood $\underline{86}$, 268-276

Riccardi A, Giordano M, Girino M, Cazzola M, Montecucco CM, Cassano E, Danova M, Ucci G, Castello A, Coci A (1987): Refractory cytopenias: clinical course according to bone marrow cytology and cellularity. Blut 54, 153-163

Ríos A, Cańtizo MC, Sanz MA, Vallespí T, Sanz G, Torrabadella M, Gomis F, Ruiz C, San Miguel JF (1990): Bone marrow biopsy in myelodysplastic syndromes: Morphological characteristics and contribution to the study of prognostic factors. Br J Haematol $\underline{75}$, 26-33

Rose C, Brechignac S, Vassilief D, Pascal L, Stamatoullas A, Guerci A, Larbaa D, Dreyfus F, Beyne-Rauzy O, Chaury MP et al. (2010): Does iron chelation therapy improve survival in regularly transfused lower risk MDS patients? A multicenter study by the GFM (Groupe Francophone des Myélodysplasies). Leuk Res 34, 864-870

Rosenberg PS, Zeidler C, Bolyard AA, Alter BP, Bonilla MA, Boxer LA, Dror Y, Kinsey S, Link DC, Newburger PE et al. (2010): Stable long-term risk of leukaemia in patients with severe congenital neutropenia maintained on G-CSF therapy. Br J Haematol 150, 196-199 Sabattini E, Bisgaard K, Ascani S, Poggi S, Piccioli M, Ceccarelli C, Pieri F, Fraternali-Orcioni G, Pileri SA (1998): The EnVision++ system: A new immunohistochemical method for diagnostics and research. Critical comparison with the APAAP, ChemMate, CSA, LABC, and SABC techniques. J Clin Pathol 51, 506-511

Sakaguchi S, Miyara M, Costantino CM, Hafler DA (2010): FOXP3+ regulatory T cells in the human immune system. Nat Rev Immunol 10, 490-500

Schanz J, Steidl C, Fonatsch C, Pfeilstöcker M, Nösslinger T, Tuechler H, Valent P, Hildebrandt B, Giagounidis A, Aul C et al. (2011): Coalesced multicentric analysis of 2,351 patients with myelodysplastic syndromes indicates an underestimation of poor-risk cytogenetics of myelodysplastic syndromes in the international prognostic scoring system. J Clin Oncol 29, 1963-1970

Schanz J, Tüchler H, Solé F, Mallo M, Luño E, Cervera J, Granada I, Hildebrandt B, Slovak ML, Ohyashiki K et al. (2012): New comprehensive cytogenetic scoring system for primary myelodysplastic syndromes (MDS) and oligoblastic acute myeloid leukemia after MDS derived from an international database merge. J Clin Oncol $\underline{30}, 820-829$ 
Schemenau J, Baldus S, Anlauf M, Reinecke P, Braunstein S, Blum S, Nachtkamp K, Neukirchen J, Strup C, Aul C et al. (2015): Cellularity, characteristics of hematopoietic parameters and prognosis in myelodysplastic syndromes. Eur J Haematol 925, 181-189

Schneider A, Hommel G, Blettner M (2010): Linear regression analysis: part 14 of a series on evaluation of scientific publications. Dtsch Arztebl Int 107, 776-782

Seita J, Weissman IL (2010): Hematopoietic stem cell: self-renewal versus differentiation. Wiley Interdiscip Rev Syst Biol Med 2, 640-653

Senent L, Arenillas L, Luño E, Ruiz JC, Sanz G, Florensa L (2013): Reproducibility of the World Health Organization 2008 criteria for myelodysplastic syndromes. Haematologica $\underline{98}, 568-575$

Shaffer LG, McGowan-Jordan J, Schmid M (Hrg.): ISCN 2013: An international system for human cytogenetic nomenclature. Karger, Basel 2013

Shenoy N, Vallumsetla N, Rachmilewitz E, Verma A, Ginzburg Y (2014): Impact of iron overload and potential benefit from iron chelation in low-risk myelodysplastic syndrome. Blood 124, 873-881

Singh ZN, Huo D, Anastasi J, Smith SM, Karrison T, Le Beau MM, Larson RA, Vardiman JW (2007): Therapy-related myelodysplastic syndrome. Am J Clin Pathol 127, 197-205

Skokowa J, Cario G, Uenalan M, Schambach A, Germeshausen M, Battmer K, Zeidler C, Lehmann U, Eder M, Baum C et al. (2006): LEF-1 is crucial for neutrophil granulocytopoiesis and its expression is severely reduced in congenital neutropenia. Nat Med $\underline{12}, 1191-$ 1197

Solé F, Luño E, Sanzo C, Espinet B, Sanz GF, Cervera J, Calasanz MJ, Cigudosa JC, Millà F, Ribera JM et al. (2005): Identification of novel cytogenetic markers with prognostic significance in a series of 968 patients with primary myelodysplastic syndromes. Haematologica $\underline{90}, 1168-1178$

Soligo D, Delia D, Oriani A, Cattoretti G, Orazi A, Bertolli V (1991): Identification of CD34+ cells in normal and pathological bone marrow biopsies by QBEND10 monoclonal antibody. Leukemia $\underline{5}, 1026-1030$

Soligo DA, Oriani A, Annaloro C, Cortelezzi A, Calori R, Pozzoli E, Nosella D, Orazi A, Deliliers GL (1994): CD34 immunohistochemistry of bone marrow biopsies: prognostic significance in primary myelodysplastic syndromes. Am J Hematol 46, 9-17 
Stang A, Poole C, Kuss O (2010): The ongoing tyranny of statistical significance testing in biomedical research. Eur J Epidemiol 25, 225-230

Starczynowski DT, Kuchenbauer F, Argiropoulos B, Sung S, Morin R, Muranyi A, Hirst M, Hogge D, Marra M, Wells RA et al. (2010): Identification of miR-145 and miR-146a as mediators of the $5 \mathrm{q}-$ syndrome phenotype. Nat Med $\underline{16}, 49-58$

Steensma DP, Bennett JM (2006): The myelodysplastic syndromes: diagnosis and treatment. Mayo Clin Proc $\underline{81}, 104-130$

Steensma DP, Bejar R, Jaiswal S, Lindsley RC, Sekeres MA, Hasserjian RP, Ebert BL (2015): Clonal hematopoiesis of indeterminate potential and its distinction from myelodysplastic syndromes. Blood $\underline{126}$, 9-16

Steidl C, Steffens R, Gassmann W, Hildebrandt B, Hilgers R, Germing U, Trümper L, Haase D (2005): Adequate cytogenetic examination in myelodysplastic syndromes: Analysis of 529 patients. Leuk Res 29, 987-993

Strom SS, Gu Y, Gruschkus SK, Pierce SA, Estey EH (2005): Risk factors of myelodysplastic syndromes: a case-control study. Leukemia 19, 1912-1918

Strom SS, Vélez-Bravo V, Estey EH (2008): Epidemiology of myelodysplastic syndromes. Semin Hematol 45, 8-13

Sugimori C, Mochizuki K, Qi Z, Sugimori N, Ishiyama K, Kondo Y, Yamazaki H, Takami A, Okumura H, Nakao S (2009): Origin and fate of blood cells deficient in glycosylphosphatidylinositol anchored protein among patients with bone marrow failure. $\mathrm{Br} \mathrm{J}$ Haematol 147, 102-112

Sullivan SA, Marsden KA, Lowenthal RM, Jupe DM, Jones ME (1992): Circulating CD34+ cells: an adverse prognostic factor in the myelodysplastic syndromes. Am J Hematol $\underline{39}$, 96-101

Swerdlow SH, Campo E, Harris NL, Jaffe ES, Pileri SA, Stein H, Thiele J, Vardiman JW (Hrg.): WHO classification of tumours of haematopoietic and lymphoid tissues. Band 2. 4. Auflage; Interlational Agency for Research on Cancer, Genf 2008

Tang G, Wang SA, Menon M, Dresser K, Woda BA, Hao S (2011): High-level CD34 expression on megakaryocytes independently predicts an adverse outcome in patients with myelodysplastic syndromes. Leuk Res $\underline{35}$, 766-770

Tavassoli M, Aoki M (1989): Localization of megakaryocytes in the bone marrow. Blood cells $\underline{15}, 3-14$ 
Thiele J, Quitmann H, Wagner S, Fischer R (1991): Dysmegakaryopoiesis in myelodysplastic syndromes (MDS): an immunomorphometric study of bone marrow trephine biopsy specimens. J Clin Pathol 44, 300-305

Thiele J, Zirbes TK, Kvasnicka HM, Fischer R (1999): Focal lymphoid aggregates (nodules) in bone marrow biopsies: differentiation between benign hyperplasia and malignant lymphoma--a practical guideline. J Clin Pathol 52, 294-300

Thiele J, Kvasnicka HM, F Facchetti, V Franco, J van der Walt, A Orazi (2005): European consensus on grading bone marrow fibrosis and assessment of cellularity. Haematologica 90, $1128-1132$

Tong W, Quintás-Cardama A, Kadia T, Borthakur G, Jabbour E, Ravandi F, Faderl S, Wierda W, Pierce S, Shan J et al. (2012): Predicting survival of patients with hypocellular myelodysplastic syndrome. Cancer 118, 4462-4470

Torlakovic G, Langholm R, Torlakovic E (2002): CD34/QBEND10 immunostaining in the bone marrow trephine biopsy: a study of CD34-positive mononuclear cells and megakaryocytes. Arch Pathol Lab Med 126, 823-828

Toyama K, Ohyashiki K, Yoshida Y, Abe T, Asano S, Hirai H, Hirashima K, Hotta T, Kuramoto A, Kuriya S (1993): Clinical and cytogenetic findings of myelodysplastic syndromes showing hypocellular bone marrow or minimal dysplasia, in comparison with typical myelodysplastic syndromes. Int J Hematol $\underline{58}, 53-61$

Tricot G, De Wolf-Peeters C, Hendrickx B, Verwilghen RL (1984a): Bone marrow histology in myelodysplastic syndromes. Br J Haematol $\underline{57}$, 423-430

Tricot G, De Wolf-Peeters C, Vlietinck R, Verwilghen RL (1984b): Bone marrow histology in myelodysplastic syndromes. II. Prognostic value of abnormal localization of immature precursors in MDS. Br J Haematol 58, 217-225

Tuzuner N, Cox C, Rowe JM, Watrous D, Bennett JM (1995): Hypocellular myelodysplastic syndromes (MDS): New proposals. Br J Haematol 91, 612-617

Valent P, Horny H, Bennett JM, Fonatsch C, Germing U, Greenberg P, Haferlach T, Haase D, Kolb H, Krieger O et al. (2007): Definitions and standards in the diagnosis and treatment of the myelodysplastic syndromes: Consensus statements and report from a working conference. Leuk Res $\underline{31}, 727-736$ 
Valent P, Orazi A, Büsche G, Schmitt-Gräff A, George TI, Sotlar K, Streubel B, BehamSchmid C, Cerny-Reiterer S, Krieger O et al. (2010): Standards and impact of hematopathology in myelodysplastic syndromes (MDS). Oncotarget 1 , 483-496

van den Berghe H, Cassiman JJ, David G, Fryns JP, Michaux JL, Sokal G (1974): Distinct haematological disorder with deletion of long arm of no. 5 chromosome. Nature $251,437-$ 438

Vardiman JW, Thiele J, Arber D, Brunning R, Borowitz M, Porwit A, Harris NL, Le Beau M, Hellström-Lindberg E, Tefferi A et al. (2009): The 2008 revision of the World Health Organization (WHO) classification of myeloid neoplasms and acute leukemia: rationale and important changes. Blood 114, 937-951

Verburgh E, Achten R, Maes B, Hagemeijer A, Boogaerts M, De Wolf-Peeters C, Verhoef G (2003): Additional prognostic value of bone Marrow histology in patients subclassified according to the international prognostic scoring system for myelodysplastic syndromes. J Clin Oncol 21, 273-282

Voso MT, Fenu S, Latagliata R, Buccisano F, Piciocchi A, Aloe-Spiriti MA, Breccia M, Criscuolo M, Andriani A, Mancini S et al. (2013): Revised International Prognostic Scoring System (IPSS) predicts survival and leukemic evolution of myelodysplastic syndromes significantly better than IPSS and WHO Prognostic Scoring System: validation by the Gruppo Romano Mielodisplasie Italian Regional Database. J Clin Oncol 31, 2671-2677

Walkley CR, Shea JM, Sims NA, Le Purton, Orkin SH (2007): Rb regulates interactions between hematopoietic stem cells and their bone marrow microenvironment. Cell 129, 10811095

Walter MJ, Shen D, Ding L, Shao J, Koboldt D, Chen K, Larson DE, McLellan MD, Dooling D, Abbott R et al. (2012): Clonal architecture of secondary acute myeloid leukemia. N Engl J Med 366, 1090-1098

Wei S, Chen X, Rocha K, Epling-Burnette PK, Djeu JY, Liu Q, Byrd J, Sokol L, Lawrence N, Pireddu R et al. (2009): A critical role for phosphatase haplodeficiency in the selective suppression of deletion 5q MDS by lenalidomide. Proc Natl Acad Sci U S A 106, 12974 12979

Wilkins BS, Porwit A: Myelodysplastic syndromes. In: Porwit A, McCullough J, Erber WN (Hrsg.): Blood and bone marrow pathology. 2. Auflage; Churchill Livingstone Elsevier, Edinburgh u. a. 2011, 303-320 
Wilson AB, Neogi T, Prout M, Jick S (2014): Relative risk of myelodysplastic syndromes in patients with autoimmune disorders in the General Practice Research Database. Cancer Epidemiol 38, 544-549

Yoshida K, Sanada M, Shiraishi Y, Nowak D, Nagata Y, Yamamoto R, Sato Y, SatoOtsubo A, Kon A, Nagasaki M et al. (2011): Frequent pathway mutations of splicing machinery in myelodysplasia. Nature $\underline{478}, 64-69$

Yoshida Y, Oguma S, Uchino H, Maekawa T (1988): Refractory myelodysplastic anaemias with hypocellular bone marrow. J Clin Pathol 41, 763-767

Yue G, Hao S, Fadare O, Baker S, Pozdnyakova O, Galili N, Woda BA, Raza A, Wang SA (2008): Hypocellularity in myelodysplastic syndrome is an independent factor which predicts a favorable outcome. Leuk Res $\underline{32}, 553-558$ 


\section{Danksagung}

Mein ganz besonderer Dank gilt Herrn Professor Dr. med. P. Ströbel, der neben der Rolle des Doktorvaters auch die des Betreuers dieser Arbeit übernommen hat. Er hat mich an das wissenschaftliche Arbeiten herangeführt und war stets erreichbar für meine Fragen. Besonders inspiriert hat mich die Begeisterung, mit der er das Fach Pathologie im klinischen Alltag wie auch im Rahmen dieser wissenschaftlichen Arbeit praktiziert. Dies war immer wieder Vorbild und Ansporn für mich.

Mein Dank gilt Frau Karin Hannemann, die mir bei allen organisatorischen Fragen kompetent, schnell und freundlich weitergeholfen hat.

Ich bedanke mich bei den Mitarbeitern der Klinik für Hämato-Onkologie, die mir die Labordaten und genetischen Analysen der Patienten zur Verfügung gestellt haben. Besonders danken möchte ich Frau PD Dr. med. J. Schanz sowie Herrn Prof. Dr. med. D. Haase für die inhaltliche Beratung bei der Planung und Umsetzung dieses Forschungsprojekts. 